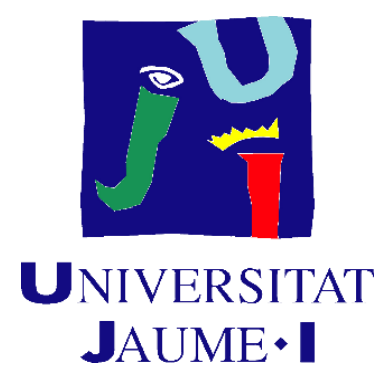

Programa de Doctorado en Ciencias

Escuela de Doctorado de la Universitat Jaume I

\title{
Complex spatial shaping of femtosecond pulses with phase-only spatial light modulators
}

Memoria presentada por Miguel Carbonell Leal para optar al grado de doctor/a por la Universitat Jaume I

Phd Student

Miguel Carbonell Leal
Supervisors

Dr. Omel Mendoza Yero

Dr. Gladys Mínguez Vega

Castellón de la Plana, diciembre de 2019 
The author and supervisors are very grateful to the University Jaume I for the financial support received for the development of this thesis through the predoctoral grant PREDOC/2015/48, and the grant E-2017-40 for the development of a 3 months doctoral stay.

The author and supervisors are also very grateful to the Servei Central $d^{\prime}$ Instrumentació Científica of the Universitat Jaume I for the employment of the facilities and equipment. 
Some people say, "How can you live without knowing?" I do not know what they mean. I always live without knowing. That is easy. How you get to know is what I want to know.

Richard Feynman. The Meaning of it All. 

A mis padres y a mi hermano.

A Ángela. 



\section{Abstract}

Controlling at will different properties of light has allowed the advancement and emergence of numerous fields of the scientific research. The possibility of arbitrarily modify parameters such as the amplitude or the phase of a laser beam has helped to adapt the light sources to the specific requirements of each user for different applications. Consequently, the development of new techniques to carry out this control has become an essential task that benefits a large number of industrial and research processes.

Spatial light modulators (SLMs) are devices that allow real time and direct control over amplitude, polarization, or phase. Generally, SLMs are capable of modulating one of these parameters. However, several devices are usually necessary to control more than one parameter at the same time, or failing that, the development of a technique that allows this control to be performed with a single device.

This thesis is focused on spatial manipulation of laser beams with a phase-only SLM. To achieve it, it is essential to arbitrarily modify the corresponding complex field with this kind of devices. In addition, it is intended to introduce new methods to improve existing encoding techniques. In that sense, the experimental implementation of a method known as double-phase is improved and optimized. With that goal in mind, the behavior of SLMs is studied in order to adapt, as far as possible, their real experimental phase response to an ideal one. Finally, in order to get results comparable to other well-known optical techniques the optimized double-phase method is used in several applications.

This thesis is focused on spatial manipulation of laser beams with a single phase SLM. To achieve it, it is essential to arbitrarily modify a complex field with this kind of devices. In addition, it is intended to introduce new methods to improve existing techniques. In that sense, the experimental implementation of a method known as double-phase is improved and optimized. With that goal in mind, the behavior of SLMs is studied in order to adapt, as far as possible, the experimental response of these devices. Finally, the optimized method is used in several applications to obtain real results comparable to other well-known optical methods. 
Among the most notable results obtained during this thesis, it is worth highlighting:

1. The experimental development and improvement of a double-phase method.

2. The introduction of a novel spatial phase multiplexed technique able to generate arrays of foci with extremely high precision in the position and peak energy of foci.

3. The development of a new calibration technique for phase-only SLMs to increase the accuracy and control capabilities of these devices.

4. The application of the optimized double-phase method to enhance both non-linear microscopic through the introduction of a complex lighting scheme and non-linear optical processes (e.g., secondharmonic generation or filamentation) by means of an accurate control of light parameters. 


\section{Agradecimientos}

No es fácil ser un ingeniero entre físicos. Y menos un informático. Por suerte el subconjunto de físicos que me ha acompañado en este camino se compone de los mejores elementos (en el buen sentido de la palabra) que se pueden encontrar. Gracias a ellos la proporción entre conocimientos adquiridos y mofas hacia el colectivo de ingenieros se ha decantado claramente hacia el lado luminoso. Gracias GROC. Estas líneas están dedicadas a ellos y, en general, a todas aquellas personas que de alguna u otra forma me han ayudado a llegar a este punto.

En primer lugar quisiera agradecer a Gladys y Omel todo el esfuerzo y tiempo que han empleado en hacer posible esta tesis. Cuando empezó esta aventura no podía imaginar la suerte que iba a tener al contar con unos directores de tesis tan dedicados como ellos. De ello han dado buena cuenta las interminables sesiones de laboratorio, los correos electrónicos recibidos a horas intempestivas y las innumerables correcciones realizadas a esta tesis. Omel me ha enseñado a desenvolverme en el laboratorio, entre otras cosas. Siempre recordaré con cierta nostalgia esos momentos diseñando y alineando sistemas, desarrollando programas o simplemente tomando medidas. Ahí he descubierto que además de un gran director de tesis, Omel es una magnífica persona... aunque sea socio del Villarreal. También estoy muy agradecido a Gladys, que ha sido parte fundamental en esta empresa. Aparte de todos los conocimientos sobre óptica que me ha transmitido y los documentos que me ha ayudado a escribir (o que directamente ha escrito ella), ha sabido aconsejarme también en el terreno personal cuando ha sido necesario. Además ha sido la encargada de pelearse con cierta escuela de doctorado para innumerables trámites (incluyendo el de que me readmitieran) y ha mediado cuando Omel y yo no estábamos de acuerdo en algo, que solía ser casi siempre. Eso le acredita como una gran mediadora. Gracias a los dos.

También quiero agradecer a Jesús, Pedro y Enrique, por sus buenos consejos en las reuniones de grupo y los artículos recomendados durante estos años, que han contribuido enormemente al desarrollo de esta tesis. A Pere, también con el gran defecto de ser socio del Villarreal, por toda la ayuda prestada tanto en el láser como en mis inicios en LabView. A Merche, nuestra crítica de cine particular y cruzada contra los microplásticos y a Lluís, siempre reunido, por auxiliarme con la docencia cada vez que les he pedido ayuda. A Vicente, por las lecciones léxicas, históricas, geográficas... y gastronómicas, y por su historia 
del gimnasio en Suecia que tan buenos ratos nos ha dado. A Vicent, por compartir con nosotros toda su sabiduría durante los almuerzos. A Dani, por enseñarme lo poco que sé sobre acústica en las reuniones de grupo. A Omar, por aquella fantástica idea del Student Chapter. A Verónica, aunque no siga mis consejos sentimentales. Tú te lo pierdes. A Yessenia, porque esta tesis no hubiera sido lo mismo sin ella. A Jorge y Rocío, por tomarse con tan buen humor lo de que les desalinease el sistema. Cosas que pasan. Y a las nuevas generaciones. Ahora que una gran generación de jóvenes dejamos el grupo, es necesaria gente inteligente, preparada, trabajadora, creativa... De momento os tendréis que conformar con Armin y Marc. También a todas aquellas personas que han tenido la suerte de coincidir conmigo durante esta aventura: Salva, Jenny, Alex, Raúl, Javi, Cristina, Susana, Eduardo, Humberto, Sergio, Wycliffe, and also to the Japanese people, who taught me a lot about their country. Thank you, Yasuhiro and students. No podía faltar aquí una mención a los madrileños y sus paellas de cangrejo, que te ayudan a valorar más a la gente de aquí.

To Valentina Emiliani and her team for giving me the opportunity to stay in her lab and their kind treatment. Special thanks to Dimitrii for mentoring me a multitude of new things. Y muchísimas gracias a Sol, por los hornazos, los castillos, las salidas por Chatelet y por ayudarme a conservar el horario español durante mis meses parisinos.

A Laura, por los chuletones y arroces al horno. A Coca-Cola, por las sobredosis de azúcar. A la persona que me hizo una ecografía del riñón, y a la persona a la que confundieron conmigo. A Paradox, por sus DLC's. A los primeros discos de Arctic Monkeys. A lo que queda de Notre Dame, por orientarme durante mis carreras en París. A Guardiola. A Messi. A los alumnos que he tenido estos años, por hacerme valorar la inteligencia. A Richard Garfield, Alan Turing, Isaac Asimov y Liam Neeson, por lo de mi faceta friki.

A mis amigos, por ayudarme a desconectar los fines de semana. Por un error en el pedido tenemos un exceso de Javis que tenemos que intentar compensar con más Migueles. Y a las chicas, por no mandarnos a paseo que es lo que deberían haber hecho hace tiempo.

Y por supuesto, al GOC por tantos y tan buenos momentos. Es difícil que olvide los viajes a Elche, incendio y baño nocturno incluidos; a Penyeta, mosquitos y baño nocturno incluidos; Barcelona, sin baño esta vez pero con bares, conciertos y visitas no planeadas; Salamanca, con su morro y sus treinta y pico 
grados de día y sus menos de diez grados de noche; las excursiones bañistas y/o senderistas a la Foradada, a Cirat y su Salto de la Novia/Viuda (nunca me acuerdo del estado civil de esa mujer), al Pozo Negro, etc. También recordaré los viajes a los Connecta y el arrocito que se comió Fernando en Sagunto o las peras al vino de Yessenia en Vila-Real, o la Yincana de Torreblanca y su secreto: Emilio uno di noi. Y por supuesto las cenas, las pre-cenas y las post-cenas, los chupitos, los dardos, los futbolines, los japoneses volcando tras dos cervezas, los cangrejos en la paella, los peluches cogidos en la máquina de la bolera, las tartas de queso, las películas de Star Wars, los consejos sobre moda, los periódicos y los bombones de Navidad, los almuerzos y comidas en la UJI, en Teruel (aunque nunca fui) o en los restaurantes cercanos a la UJI, los FIBs, los Chilis y muchas otras cosas que ahora mismo no recuerdo, pero que sin duda han influido (no necesariamente para bien) en el devenir de esta tesis.

Y a título personal, a Eva, por su apoyo informático dentro del grupo. Gracias a ella ha sido más sencillo sobrellevar la incomprensión. PD: un saludo a Nala. Aún me debes unas chanclas. A Esther, por acogernos en su casa y descubrirme el arroz con costra. También por soportarnos durante largos años durante las comidas. Encajabas bien las bromas y por eso eras un blanco fácil, aunque te pusieses dorada en verano. $Y$ por salvarme en Salamanca de aquel que pensaba que mi culito era un reloj. Persigue tus sueños, lucha por ese sofá. Ángel, gracias por cederme el escritorio desde el que escribo estas líneas. Además de heredar este sitio me hubiera gustado heredar tus iCacharros y tus bíceps, pero no se puede tener todo en esta vida. Por supuesto el termostato está a +3. A mi mejor compañero de despacho Carlos, por el mosquitocidio, por su fino paladar y por ayudarme a defender el honor de Castellón frente a las injurias de Fernando. Por los arroces nocturnos, y también por tus dotes de orientación. Que la luz te guíe. Lo vas a necesitar. A Fernando, por aguantarme en París, sobre todo ese maravilloso primer fin de semana. Creo que toqué fondo desayunando calamares. Y bueno, también por el striptease de Barcelona, por los puzles de Amazon y por cargar el peso de LA CRUZ.

A mis tíos y primos, que aunque cada vez nos veamos menos, siempre los tengo muy presentes. A Ángela, por aguantarme, que ya tiene su mérito. Por comprenderme y apreciarme como soy y por convertirme una persona mejor cada día que estamos juntos. A mi hermano, del que estoy muy orgulloso y sólo puedo decir cosas buenas. Hemos peleado, hemos discutido, hemos roto cosas (incluyendo mi brazo), pero al final siempre hemos vuelto a la 
normalidad en tiempo récord. Y por último, y por ello más importante, a mis padres. Por cuidarme, por darme todo lo que he necesitado y por educarme como lo han hecho. Esta tesis es el final de esa educación.

En definitiva, gracias a todos por estos fantásticos años. 


\section{Contents}

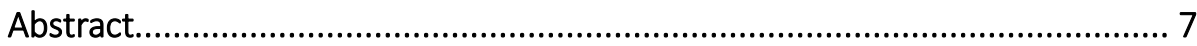

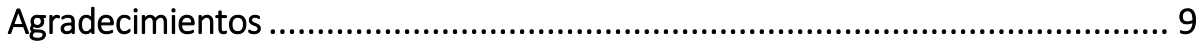

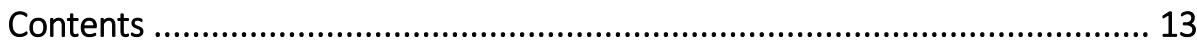

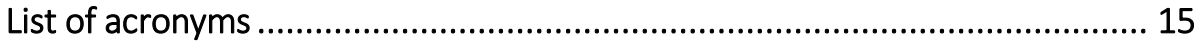

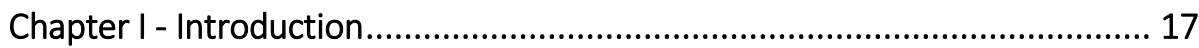

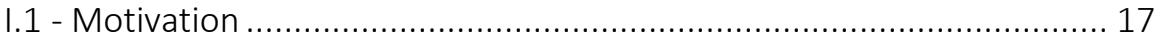

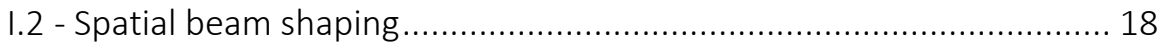

1.3 - Beam shaping of femtosecond pulses ............................................ 23

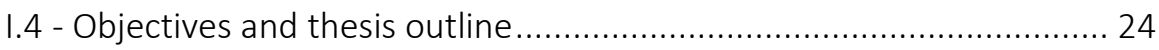

Chapter II - Programmable spatial light modulators ..................................... 27

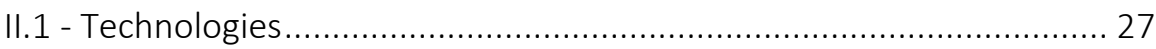

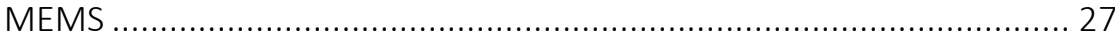

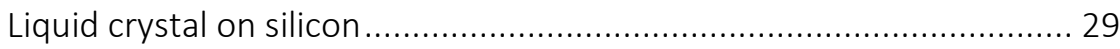

II.2 - Common experimental issues......................................................... 33

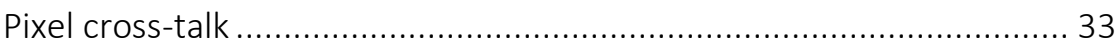

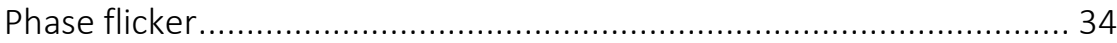

II.3 - Characteristics of spatial light modulators …….............................. 34

II.4 - Calibration of spatial light modulators.............................................. 37

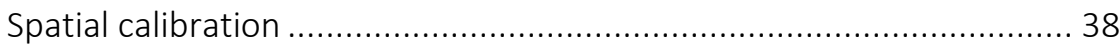

II.5 - Diffraction-based phase calibration of SLM with binary phase Fresnel

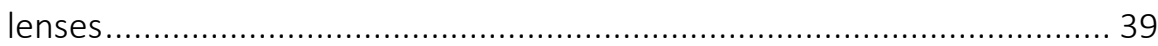

Chapter III - Beam shaping using phase-only SLMs ......................................... 45

III.1 - Conventional encoding techniques ................................................ 45

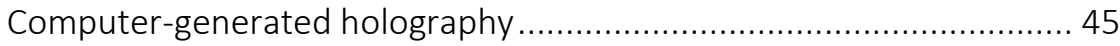

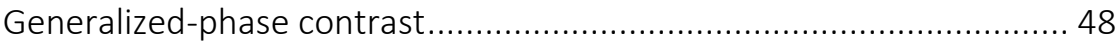

III.2 - Shaping techniques for focusing diffractive elements .................... 50

III.3 - Diffractive control of 3D multifilamentation................................... 51 
Chapter IV - Complex field encoding........................................................... 63

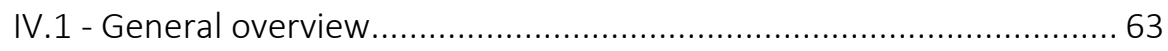

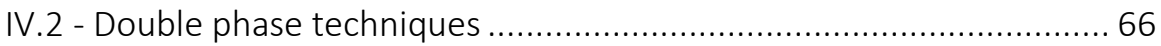

IV.3 - Shaping the amplitude and phase of laser beams ......................67 67

Chapter V - Applications of complex field encoding ................................... 79

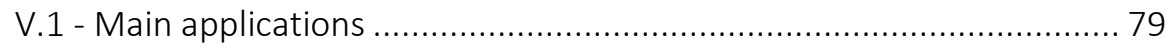

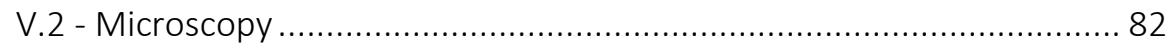

V.3 - Generation of 3D foci distributions with complex Fresnel holograms 85

V.4 - Micrometric complex illumination patterns for non-linear microscopy

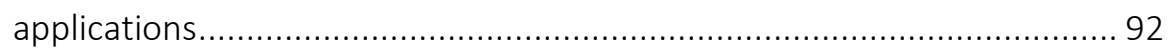

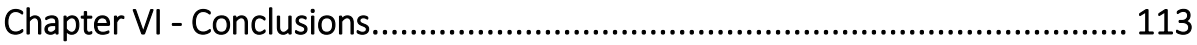

VI.1 - Summary of contributions ............................................ 113

VI.2 - Future work ............................................................ 115

Appendix A: Challenges and recent hints of phase-only SLMs .................. 117

Resumen en castellano......................................................................... 121

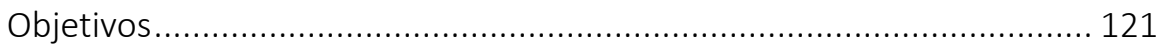

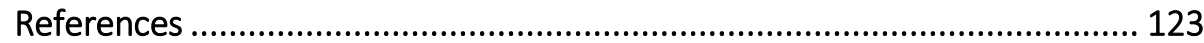




\section{List of acronyms}

AOM Acousto-Optic Modulators

BBO Type $1 \beta-\mathrm{BaB}_{2} \mathrm{O}_{4}$ crystal

BPFL Binary Phase Fresnel Lens

CCD Charge-Coupled Device

CGH Computer-Generated Holography

CMOS Complementary Metal-Oxide-Semiconductor

$\mathrm{CPI} \quad$ Common-Path Interferometer

DM Deformable Mirror

DMD Digital Micromirror Device

DOE Diffractive Optical Element

DPE Diffractive Phase Element

DPH Double-Phase Hologram

DVI Digital Visual Interface

ECB Electrically Controlled Birefringence

FDE Focusing Diffractive Element

FLCD Ferroelectric Liquid Crystal Display

GSA Gerchberg-Saxton Algorithm

HDMI High Definition Multimedia Interface

IFTA Iterative Fourier Transform Algorithm

LC Liquid Crystal

LCD Liquid Crystal Display

LCoS Liquid Crystal on Silicon

LUT Look-Up-Table 
MEMS Micro-Electro-Mechanical-System

ODT Optical Diffraction Tomography

PA Parallel Aligned

PCF Phase Contrast Filter

PSF Point Spread Function

PWM Pulse Width Modulation

RMSE Root-Mean-Squared-Error

ROI Region-Of-Interest

SCMM Spatial Cross-Modulation Method

SHG Second-harmonic Generation

SLM Spatial Light Modulator

TN Twisted Nematic

VAN Vertical Aligned Nematic

WGS Weighted Gerchberg-Saxton

WGS-PC Weighted Gerchberg-Saxton with Phase Control 


\section{Chapter I - Introduction}

\section{I.1 - Motivation}

Photonics is an indispensable part of our lives. From communications, to medical tests, through electronic displays, many aspects of our daily lives have improved thanks to the research carried out in this field. In fact, the European Commission considers it as one of the key enabling technologies because of its importance in a wide range of industrial sectors [1]. This means that photonics is currently one of the priority technologies, and will continue to be so at least in the near future. Its current impact is undoubted: for instance, communications and information as we understand them today could not be possible without photonics. Nowadays, fiber optic is essential for the rapid transmission of information and it is expected that this will continue in the coming years to meet the ever-increasing demands of the telecommunication industry. In addition, healthcare is largely benefited from photonic technologies. Both for the detection of diseases and for their treatment, photonics provides innovative tools to help the development of new cures and early-stage diagnosis. It is estimated that the impact of photonics in this field represents an economic saving of as much as 20\% [2]. In addition, the number of elder people is expected to increase progressively in the coming years. In particular, the number of people in the UE over 65 will have grown by $70 \%$ in 2050. In the case of people over 80 , this value raises to $170 \%$. This group of people is one of the most vulnerable and dependent on health care, increasing the importance of this field in the near future. Electronic displays is another technology that depends largely on the ability to control the light. Information, communication, and navigation are some of the multiple fields that employ devices which require displays. These displays seek to offer more and more features. Additionally, it is expected that in next years, newly created technologies will be consolidated. Augmented reality, virtual reality or microdisplays in glasses will become part of our society requiring new milestones both in resolution and in size reduction. Relevance of photonics is reflected at the economic level. According to the European agency Photonics21, photonics employs 300,000 people and generates $€ 69$ billion in Europe alone, and the global market is expected to reach $€ 615$ billion in 2020 [3]. All these data show that photonics is not only an important part of our lives, but also of our economy. 
With an increasing demand on performance for photonics-based devices, most applications are faced with problems related to inadequate manipulation of the light beam, housing a barrier for a wider settlement. Smart control over light propagation is an important question that undoubtedly needs to be addressed to overcome the current barriers and take full advantage of the potential of optical methods. Digital spatial light modulators are devices that provide fine-grained control of light, allowing the creation of light patterns with user-controllable characteristics. The devices consist of an array of pixels, each of which can individually control the phase or amplitude of light passing through it or reflecting off it. Millions of pixels provide numerous control freedoms for tailoring the wavefront of light in a programmable manner paving the avenue to overcome the limitations of current photonics technologies, e.g. for healthcare and laser manufacturing. Despite the large prevalence of spatial light modulators in current photonic devices, there is a need to enhanced light control and decrease the operational burden over photonic tools extending significantly the range of applications.

\section{2 - Spatial beam shaping}

Photonics is currently considered as one of the key enabling technologies. That means that there are many industrial and scientific applications that benefit from it and, as a result, controlling the physical behavior of light is a topic that is currently highly demanded and relevant.

The typical Gaussian laser beam available at the output of a standard laser is not the most appropriate for many applications. For this reason, it is interesting to redistribute the irradiance and phase of a laser beam. This process is known as beam shaping and is commonly achieved by using either static or dynamic diffractive optics. A classic example of this process is to transform a Gaussian beam into a beam of uniform irradiance (Fig. I.1). 


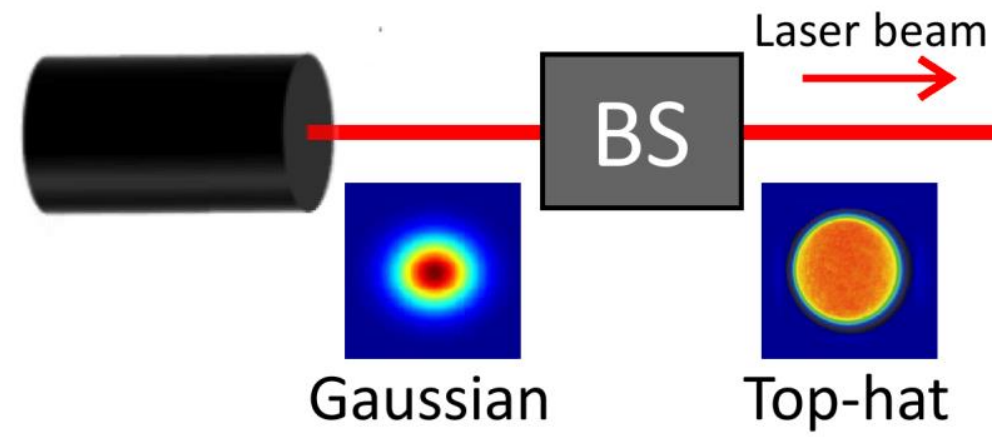

Fig. I.1 - Example of a beam shaper (BS). The Gaussian beam obtained at the output of a laser source is redistributed to obtain a uniform irradiance beam.

Some industrial applications benefit from a uniform irradiance pattern with a flat top profile and very steep edges (Fig. I.2). This shape is commonly known as flat-top beam or top-hat beam. In contrast to a Gaussian beam, where the intensity decays from a maximum level of intensity near the beam axis to zero, the intensity of a top-hat remains constant. Some fields, such as material processing, benefits from a beam of uniform intensity that allows precise control of the process [4]. Other useful patterns for other applications include shaped lines, rings, and arbitrary array patterns.

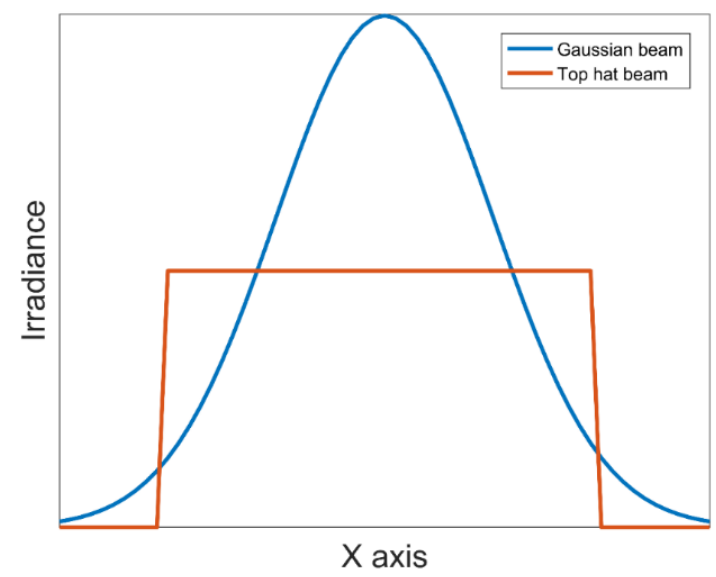

Fig. I.2-Comparison between the irradiance profile of a Gaussian beam and a top-hat beam along the $x$-axis. 
Nevertheless, beam shaping is not only restricted to 2D distributions of light. Non-diffracting beams are used in some applications to improve certain characteristics. For example R. Lu et al. [5] generate a Bessel beam to include it as the illumination source of a two-photon laser-scanning microscope. In that way, they are able to scan not just a surface but a whole volume, improving the temporal resolution of the system.

Regarding the techniques used to carry out the beam shaping, there are several ways to define the complex distribution of the light beam. For example, in the case of non-diffractive beams, these can be generated by an annulus located in the back focal plane of a lens. As this annular aperture is the Fourier conjugate of the Bessel beam, this configuration generates a Bessel mode [6]. However, it can also be generated in other ways, for instance by using interferometric techniques [7] or with an axicon [8]. An axicon is a conical shaped lens that produces a certain phase shift over the incident beam in a way that all the waves are inclined at the same angle to the optical axis, that is, the beam is formed by waves that propagate on a cone shape and interfere to generate the Bessel mode profile (Fig. I.3). This results in the generation of a narrow beam of light along the optical axis [9]. Both axicon and annulus can be obtained by different ways either statically or dynamically.

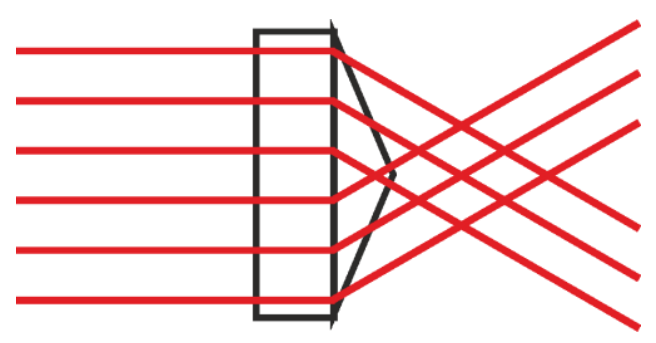

Fig. I.3 - Schematic of an axicon lens

Static optical components are created to accomplish a certain target whereas dynamic components are characterized by being able to change their behavior in real time to perform multiple goals. An example of a static element is a diffractive lens manufactured with lithographic techniques. The main advantage of this method is the high accuracy with which they can be generated, with errors of the order of tenths of microns. The main drawback lies in the impossibility of being modified once it has been generated. That is why the specifications of the diffractive lens must be clear before making it, because if an error is made, a new one must be constructed. In addition, if the 
specifications of the experiment change, the lens is no longer useful. An example of dynamic component is an SLM. An SLM is a device able to manipulate light in two dimensions by using a matrix of pixels. An electronic controller manages each pixel independently allowing spatially varying modulation over a beam of light. Such control allows the programmable generation of user-defined light distributions to fulfill multiple purposes. Two main SLM technologies are employed for this purpose: micro-electromechanical system (MEMS) based arrays or liquid crystal (LC) spatial light modulators. MEMS devices contain moving parts and are characterized by a very high switching speed, generally at multi-kHz frequencies. Two examples of MEMS devices are digital micromirror devices (DMDs) and deformable mirrors (DM). On the other hand, LC SLMs are based on the special properties of liquid crystals and do not contain moving parts. This is associated with maximum laser energy utilization and high throughput potential [10].

Hence, SLMs are used massively as dynamic components due to the option to encode different patterns. Following the previous example, it would allow the diffractive lens to be modified several times per second; however, the size of the pixel (about 6-8 $\mu \mathrm{m}$ ) limits the resolution of the encoded lens. This example is applicable to different situations where a specific diffractive optical element (DOE) is required and can be generated by encoding it onto a programmable device or physically built as an optical element. Here, other details must be considered. For example, the economic cost. A wide range of SLMs with notable differences in price is available in the market. Although the economic cost of a high-end or mid-range device could be more expensive than a static element, e.g. a commercial photomask, the photomask can only encode a certain pattern while the SLM can encode any desired pattern. In the long term, the SLM can be used in many different applications for several purposes, being a good investment over time compared to static elements. In particular, SLMs are employed in a myriad of applications as microscopy [11], holographic imaging [12], laser micro-processing [13], optical beam photolithography [14], quantum information [15], and holographic optical trapping [16], among others. 


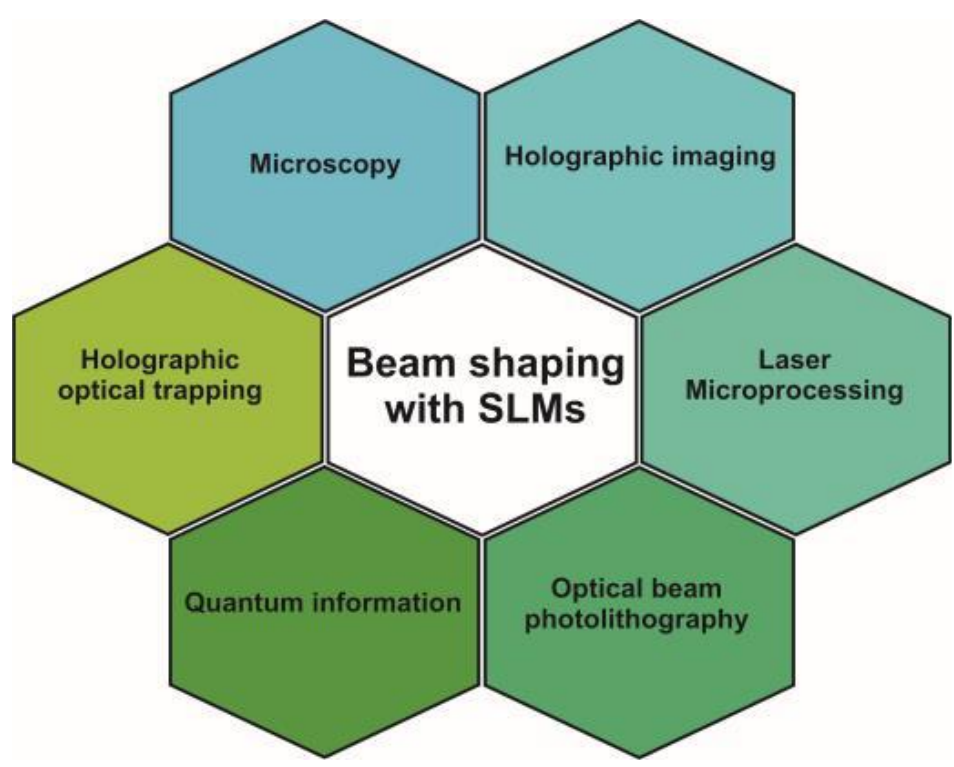

Fig. I.4 - Applications of beam shaping with SLMs.

To achieve these multiple objectives it is necessary to manipulate the laser beams in an appropriate manner. In that sense, we talk about amplitude modulation, phase modulation, and simultaneous amplitude and phase modulation (or complex modulation). Depending on the device used, each modulation may be more or less simple to obtain. For example, obtaining binary amplitude modulation with a DMD is trivial, it is enough to send a binary pattern to this device and form an image of the DMD plane. However, this procedure has important limitations. For high-power demanding application (e.g., laser micro-processing) it is better to confine all available light in one or more foci rather than getting them by image formation, since most of the light would be lost. In these situations, wavefront modulation obtained with a phase-only SLM is a higher versatile tool. For this reason, it is necessary to use techniques that allow us to obtain not only the desired patterns, but also the parameters that each application requires [17].

A classic example of that is the encoding of DOEs into SLMs to obtain arbitrary light distributions reconfigurable in real-time. This allows to simulate the behavior of classic diffractive optical elements. Another example is holography, where the hologram can be optically captured in a traditional way or computer generated. Furthermore, computer generated holograms (CGHs) can be encoded into a SLMs to manipulate laser beams. The common procedure is to define the requested amplitude or phase pattern on the target 
plane and calculate (e. g. by means of the Fourier transform) the hologram that reconstruct the desired pattern. Among other methods, iterative algorithms are widely employed for that purpose. Gerchberg-Saxton (GSA) [18] and Yang-Gu [19] algorithms are two examples of classical iterative methods to get approximate solutions to the desired pattern. Other techniques owing to achieve an accurate amplitude pattern have been developed. This is the case of generalized phase contrast (GPC) [20], which is basically an interferometric method employed to visualize phase variations as intensity changes at an output plane.

\section{3 - Beam shaping of femtosecond pulses}

A femtosecond laser is a light source that emits electromagnetic pulsed radiation with durations on the domain of femtoseconds $\left(10^{-15} \mathrm{~s}\right)$. Therefore, it falls into the category of ultrashort pulsed lasers. Femtosecond pulses are extremely short events; in fact, they are among the shortest processes created by humans. Some natural processes also happen in very short periods of time (e.g. synaptic transmissions). To time-resolve these kind of events, a faster or similar procedure is needed. To generate femtosecond pulses, multiple waves of different frequencies are coherently summed. Thus, the resulting wave has a broad spectral bandwidth. The general rule is, the shorter the pulse the larger the spectral bandwidth. A femtosecond pulse can have a spectral width of dozens of nanometers while the spectrum of a continuous laser is almost monochromatic. The addition of the multiple waves in a short pulse also results on the addition of their low average energies into a high peak power. Amplified laser systems can reach peak powers of the order of gigawatts (GW). Finally, the coherence length of femtosecond pulses is short compared to conventional laser sources. Some applications like optical coherence tomography are benefited from the reduction of this parameter [21].

Similar to conventional laser beams, femtosecond pulses increase their possibilities of use when being manipulated to meet user-defined specifications. Due to their nature, the femtosecond beams can be modified in the spatial [17], the spectral [22] and/or the temporal domain [23] to fulfill the particular requirements of an application. In this thesis, we focus on programmable spatial beam shaping. Among the most noticeable applications of femtosecond spatial beam shaping, one can find optogenetics [24], ultrafast processing of materials [25], microscopy [26], non-linear optics [27], and more. 


\section{I.4 - Objectives and thesis outline}

The proposed research is driven after the observations of shortcomings in current state-of-the-art. Over the past decades, powerful optical waveform synthesis methods have been developed and demonstrated a strong impact as experimental tools providing unprecedented control over ultrafast laser waveforms. However, spatial optical manipulation of ultrashort pulses according to user specifications is usually achieved only over the output irradiance or over the full complex field thorough approximated solutions. We have detected that there is a lack of simple and versatile encoding techniques to precisely manipulate complex ultrafast beams. In this thesis, we address this challenge under several perspectives, starting from a simple method to calibrate an SLM and finishing with real applications of shaped ultrafast beam. Our main aim is to develop versatile tools based on phase-only SLMs that widespread the powerful optical waveform synthesis of femtosecond beams and extend its applications. Specifically, the objectives of this thesis are the following:

1. Implement a method to encode complex fields from a single phase mask sent to a phase-only SLM.

2. Apply this encoding method in real applications that may involve tangible improvements over existing techniques.

3. Compensate for possible experimental problems of phase-only SLMs that reduce the efficiency and accuracy of the developed method.

4. Introduce alternative encoding strategies for modifying laser beam parameters.

This memory is structured as follows:

In chapter 2, an analysis on the different types of devices capable of modifying the properties of light is carried out, going deeper into the devices based on liquid crystal displays that only modulate the spatial phase. In order to understand the real behavior of a laser beam after impinging onto a SLM, some of the unwanted effects that can occur when working with these devices will be described. Next, it is explained how to mitigate, some of these problems. With that in mind, it is explained why it is important to periodically calibrate a device, and some examples of how to do it are presented. Lastly, it includes a new method to calibrate an SLM in an easy way and with an extremely simple setup is presented. 
In chapter 3, the concept of beam shaping and how to obtain an arbitrary irradiance pattern over a certain plane is introduced. In that context, a brief introduction is included containing some of the most used techniques to generate an arbitrary irradiance pattern: iterative algorithms, phase contrast methods, and focusing diffractive elements. Finally, a method able to generate three-dimensional distributions of foci in arbitrary positions is described.

Chapter 4 takes another step forward and deepens into the techniques capable of shape not only the irradiance, but the complete complex field. Special emphasis is placed on the so-called double phase methods, including an indetail description of the theory related with the technique. To conclude, a new version of a well-known double-phase method [28], is presented, demonstrating its usefulness with pulsed infrared illumination, and handling of common experimental issues to maximize efficiency.

Chapter 5 summarizes the most common applications of the encoding methods that allow controlling the phase and amplitude simultaneously. Subsequently, two applications of the encoding method are detailed. The first one uses Fresnel holograms to generate a set of foci with micrometric precision. The encoding technique ensures an optimal codification of both amplitude and phase of the complex field, allowing to generate an illumination pattern with full control over the transversal and axial positions. The second application employs the encoding method to generate wide-field arbitrary light distributions, controlling amplitude and phase of pre-defined regions-ofinterest. In both cases, the illumination patterns have been used to generate non-linear effects with potential application to non-linear microscopy.

Chapter 6 sets out the conclusions of this thesis. It summarizes the contents of this manuscript, list the main contributions of this work, and detail some of the future perspectives of double-phase methods. 



\section{Chapter II - Programmable spatial light modulators}

In this chapter, a brief review about some spatial light modulating devices is presented. Then, we focus on phase-modulation devices, describing their characteristics, some of their recurring problems and how to calibrate them to solve part of these problems. Finally, we present a novel technique to do this calibration with a remarkably simple method.

A device that is able to modulate amplitude, phase or polarization of light waves spatially and/or temporally is considered an SLM. There is a wide variety of different devices depending on the technology with which they are manufactured. According with this criterion, we can classify SLMs on different groups. In this thesis, we will focus on the devices based on micro-electromechanical-systems and especially the devices based on liquid crystal screens. In the next paragraphs, we will see how do they work and some of their most typical uses.

\section{II.1 - Technologies}

\section{MEMS}

MEMS is an incredibly general term that includes a lot of different devices, commonly assembled with moving parts, developed for applications including biomedical [29], inkjets [30], microphones [31], gyroscopes and accelerometers [32], and of course, optics.

For optics both single-mirror and multi-mirror arrangements can be found [33]. Included in the single-mirror group, galvanometric mirrors are among the most common micro-devices. They are made of an optical mirror mounted over a galvanometer-based scanning motor and a sensor that gives feedback about the position of the motor to the controller. This arrangement allows one-dimensional scanning. Another widespread configuration consists of two mirrors mounted over two different motors allowing two-dimensional scanning.

Galvanometric mirrors are considered as single mirrors as the whole beam is reflected following the same trajectory. On the other hand, other devices as deformable mirrors and arrays of mirrors own a large number of actuators allowing individual control of each of them. In that way, it is possible to apply a different angle of reflection on each one of these mirrors/actuators. Deformable mirrors are reflectors with a membrane whose shape can be 
conveniently deformed by using an array of actuators. Each actuator covers a region of the membrane, so that the more quantity of them, the higher resolution they afford. These devices are widely used in wavefront shaping applications due to their versatility and high resolution wavefront correction [34].

There are other kind of active matrix-based devices based on different technologies. Probably one of the most successful is the digital micromirror device from Texas Instruments, which is basically an array of millions of highly reflective mirrors that swap between two tilted positions $\left(+12^{\circ}\right.$ and $\left.-12^{\circ}\right)$ with respect to the normal surface of the DMD. A complementary metal-oxidesemiconductor (CMOS) board controls all mirrors.

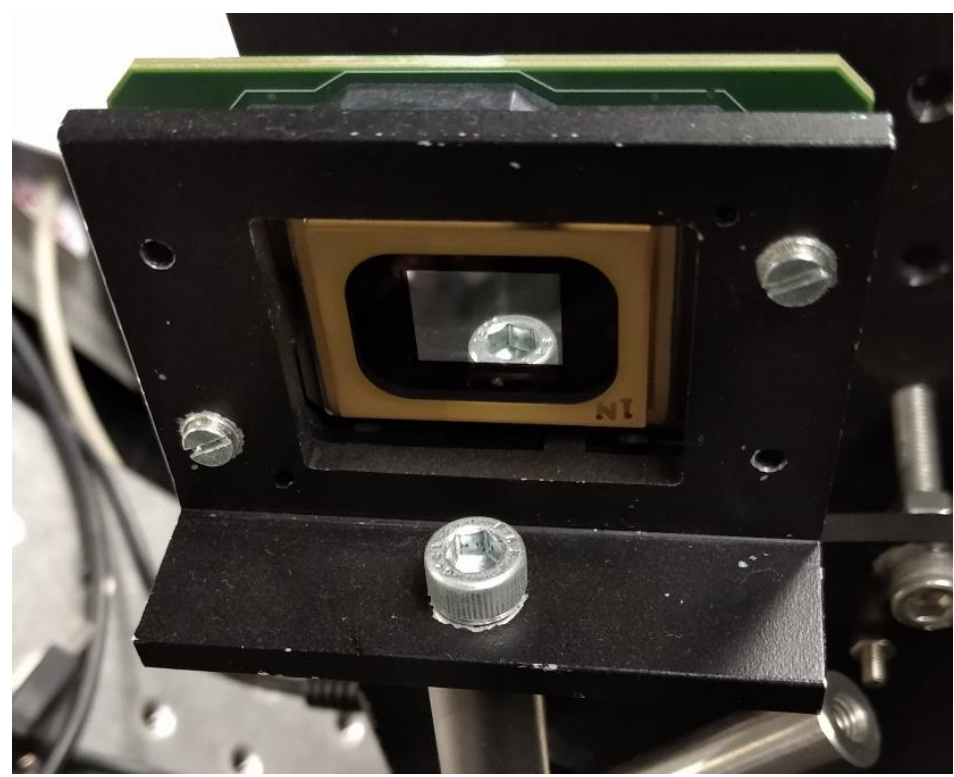

Fig. II.1 - Picture of the screen of a DMD.

Thanks to their relative fast pattern rates (in the order of tens of $\mathrm{kHz}$ ), polarization independence and the large spectral response of the mirrors, DMDs shine on certain applications like laser beam shaping [35] or pattern representation. Using its ability to shape light, different works aimed to generate Laguerre-Gaussian modes [36] or non-diffractive beams [37] have been presented.

Prior to discussing about LC SLMs, it is important to comment about other devices not included on these groups, but still very relevant in some fields. This is the case of acousto-optic modulators (AOM). AOMs are devices based on 
the acousto-optic effect. Light is propagated through a transparent crystal (or glass) attached to a piezoelectric transducer. This piezo generates a strong oscillating electrical signal and excites a sound wave, which travels through the crystal leading to a change of the refractive index of the medium. This device can be employed for several purposes: for instance, as an intensity regulator, or as a deflector able to quickly change the position of a beam in one direction. In that way, it is possible to employ them to scan one sample over two dimensions by employing two of these devices [38,39]. Recently, it has been demonstrated its utility as a two-dimensional spatial wave front shaper [40].

\section{Liquid crystal on silicon}

Some materials present a behavior halfway between conventional liquids and solid crystals. Their molecules are oriented according to one direction (like a crystal) but they have the ability to move or rotate like a liquid. They are known as liquid crystals. Among other applications, these materials are widely used in video projection and optoelectronics. A liquid crystal display (LCD) contains a $\mathrm{LC}$ layer that is employed as a birefringent material with the option of changing the birefringence index depending on the voltage applied to the LC cells. A birefringent material is an element whose refractive index depends on the polarization and propagation direction of light. In case of crystals with a single optic axis, the ordinary axis (perpendicular to the optic axis) presents a certain refractive index whereas the extraordinary axis, which is in turn perpendicular to the ordinary axis, presents a different one. Liquid crystal on silicon (LCoS) SLMs take advantage of birefringence to provide an electrically variable index of refraction for light polarized along the extraordinary axis, while light polarized along the ordinary one is not changed.

Liquid crystals can be found on three different phases: smectic, nematic, and cholesteric. Here we are going to focus on nematic LCS, since they are the most relevant for achieving the goals of this thesis. In nematic LCs, molecules are oriented in a certain way, but the positions they occupy within the volume of the material are random. There are different ways to modulate light depending on the structure of the LC layer. Several structures are employed in nematic LCoS devices, such as twisted nematic (TN), vertical aligned nematic (VAN), and parallel aligned (PA), among others. On the TN mode, molecules are typically aligned on a $90^{\circ}$ twist helical structure. This means that the orientation of the molecules varies $90^{\circ}$ between the bottom and the top of the LC cell. This structure can be altered by applying an external electric field, so that a controlled change can be generated both in the birefringence of the LC and in the polarization of the incident light (Fig. II.2). 

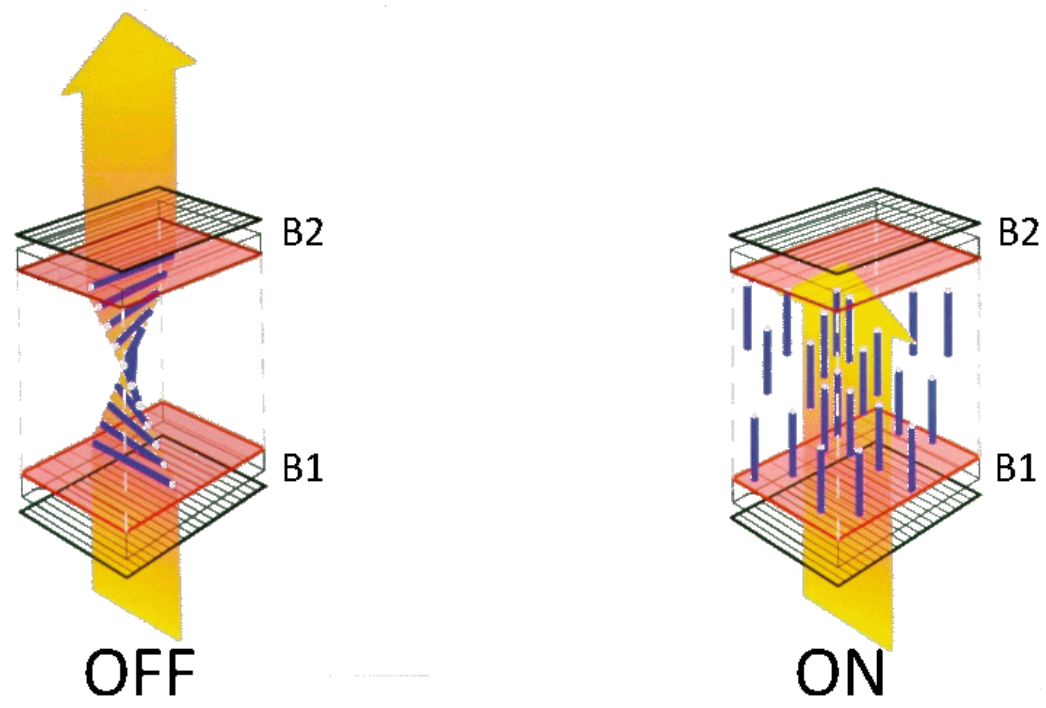

Fig. II.2 - Example of a LC TN cell. Left part shows the alignment of the molecules (blue lines) where no field is applied. Right part shows the alignment where an electric field is applied. B1 and B2 represent the borders of the cell. The yellow line represents light. As two crossed polarizers are placed in B1 and B2, in left image light is able to pass by the second polarizer thanks to the change in the polarization caused by the molecules. In right image, no change in the polarization is caused and light is unable to go through the second polarizer. Image adapted from [41].

In VAN mode, LC molecules are aligned perpendicular to the surface of the alignment layer. In this way, when no field is applied, no changes in the direction of the polarization are originated. Placed between polarizers, almost all light is blocked by the analyzer resulting in a quasi-pure black color. For that reason, this mode is widely used in displays, obtaining a high contrast ratio. When an electric field is applied, molecules tend to align following it changing the polarization of light and allowing passing through the polarizers. In PA mode, molecules remain parallel to each other and to the alignment layer if no electrical field is applied. When the field is applied, the molecules tend to align in the same direction causing a change in the birefringence of the medium. A relevant difference between VAN and PA modes is the slower response time and higher threshold voltage needed in VAN to obtain the same phasemodulation [42]. Hence, PA mode is widely used in phase-only devices. Finally, 
ferroelectric LCDs (FLCD) are also employed. These devices use molecules in the smectic phase instead of the nematic one. In this case, the position of the molecules is not entirely random, but they line up forming layers. Typically, FLCDs allow positioning the molecules in two stable states extremely fast compared to the previous types. In this way, they offer a fast but only binary modulation. Nevertheless, to achieve non-binary modulation some strategies have been developed [43].

The first devices based on LCD were passive matrix displays, with the capacity to control dozens of cells, and were used to design low-power watches and calculators. This evolved to active matrix systems, since they allow to control millions of pixels independently, what made them very useful for front- and rear- projection systems. LCoS devices were also originally created as displays for video projection. Reflective LCoS technology is an active matrix LCD that uses a liquid crystal screen placed over a silicon backplane. This backplane applies an electric current on the liquid crystal molecules to control their orientation, allowing the device to modulate the phase and polarization of light directly. The backplane also has a high reflectivity surface, so light that passes through the screen impinges onto the surface and is reflected back.

These kind of cells can modulate amplitude by using a couple of polarizers and a polarizing beam-splitter cube. The first polarizer is employed to linearly polarize the incident light field, while the second one is used to analyze it after passing through the LC cell. This second polarizer should be oriented perpendicular to the initial polarization of light. The polarizing beam-splitter cube is employed to separate the incident light from the reflected light. To achieve the amplitude modulation, the LC cell rotates the polarization of the incident light allowing it to be transmitted through the second polarizer. Thus, by controlling the polarization it is possible to define the level of light attenuation produced by the second polarizer. To modulate amplitude with PA cells, the extraordinary axis of the LC molecule should be aligned $45^{\circ}$ to the direction of the light polarization, allowing a maximum polarization rotation of $90^{\circ}$. Phase modulation can also been performed. For instance in PA, it can be achieved if the extraordinary axis and the direction of the light polarization are aligned. In this case, the LCD acts as a voltage controlled variable retarder. This is commonly known as ECB (electrically controlled birefringence). Further information can be found in $[42,44-46]$.

Although it is possible to modulate amplitude and phase with an LCoS device, one can notice that each modulation requires a mutually exclusive alignment 
of the polarization over the extraordinary axis. Therefore, since both modulations are not independent, it is not possible to do it directly with a single LC device. This limitation makes necessary to develop some approaches to achieve full complex modulation with only one device, as will be shown in next chapters.

All devices based on the technologies shown before have their own advantages and disadvantages. LCoS SLMs are widely used for optical modulation due to their high optical efficiency and high number of independent pixels at the price of a low refresh-rate (typically from 10 to 200 $\mathrm{Hz}$ ). On the other hand, DMDs offer a solution to this problem with devices operating in the order of $\mathrm{kHz}$; however, the disadvantage in this case comes from the binary modulation on the amplitude provided by them. Most LCoS SLMs are managed by a controller that supports 8-bit phase images, allowing 256 gray levels. This means that the device is capable of applying 256 different states. DMDs, in contrast, operate as purely binary amplitude modulators being more suitable for amplitude-only modulation. Other MEMS based devices as deformable mirrors share some characteristics with DMDs, for instance its frame rate is similar to the one mentioned for DMDs. Its main drawback lies in the number of actuators. Mid-range deformable mirrors have hundreds of actuators, which implies a considerably lower number of degrees of freedom with respect to the other technologies, which offer millions of them.

So far, several alternatives have been described. During this thesis, phase-only LCOS SLMs have been selected for being the ideal device to complete the proposed objectives. If we are looking for complex modulation with a single device, LCOS SLMs are the ideal devices because they allow achieving this objective with efficiencies higher than other devices. Additionally, our interest is focused on encoding the complex field with the highest possible efficiency, accuracy and resolution, and that is achieved using LCOS SLMs. As these kind of devices are selected for this thesis, we will go deeper on some of the most common experimental troubles that one faces when working with them. 


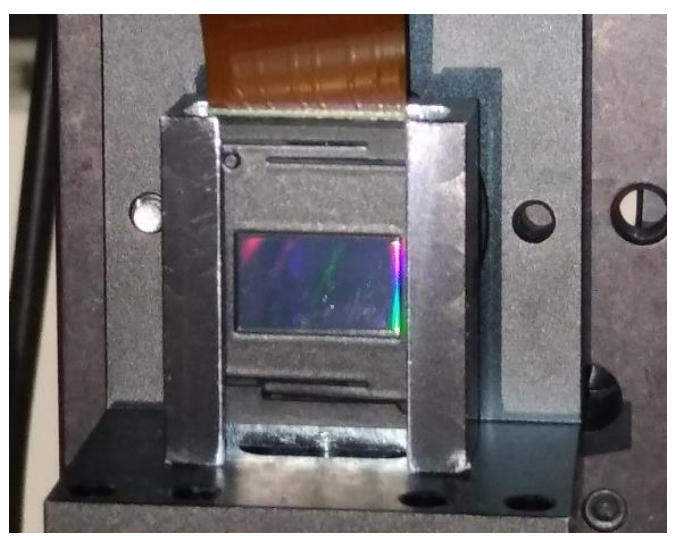

Fig. II.3 - Picture of the screen of a phase-only SLM.

\section{II.2 - Common experimental issues}

Although LCoS SLMs allow complete control over the phase of a light wave, they are not ideal devices and show some unwanted effects that can slightly modify the desired phase. Here we will discuss two fairly recurring problems: pixel cross-talk and phase flicker, which are responsible of reducing the phase modulation response.

\section{Pixel cross-talk}

It is usually assumed that the phase generated by each of the pixels of the LC is independent of the neighboring pixels. Actually, this is not the case for two reasons. First, each one of those pixels receives a different voltage to obtain the desired phase retardation, but as these devices suffer from crosstalk between contiguous pixels, gradual voltage changes appear at the frontier between them. This is caused because the electric field generated by each pixel is not limited to the area of that pixel, and the molecules of contiguous pixels are influenced by it. Secondly, the molecules present in the LC layer generate elastic forces that prevent quick spatial variations in the phase response of the SLM [47].

To compensate these effects, several techniques have been developed. For instance, one proposal lies in the use of polymer walls to isolate each pixel and separate them, keeping each electric field into the pixel area and eliminating gradual changes in the field [48]. Another possibility is to try to minimize the effect by varying the voltage profile applied to the cells [49]. In holography, some works have been reported using optimization algorithms considering these effects to try to improve the overall result [50]. We have studied this 
problem and proposed an alternative strategy to mitigate its effects. Further details about this work can be found in [51].

\section{Phase flicker}

One may think that during the entire time that a frame is active in, for example, a phase-only LCOS SLM, the encoded phase is constant. Actually, during that time several phenomena happen making it not so. First, it is important to explain how the gray level control is performed. Assuming that a model with an 8-bit resolution is used, the user has a range of 256 gray levels that he/she can use. The device receives an input with those 256 gray levels encoded in a signal that can be analog or digital. In the first case, that signal is used directly for the representation of the level of gray in each of the LC cells. In the second case, things are more complicated. The gray level is encoded in a binary signal using a technique called pulse width modulation (PWM). This technique generates, for each analog gray level of the signal, a binary train of pulses with a frequency in the order of the $\mathrm{KHz}$. The pixel of the $\mathrm{LC}$ screen interacts with each one of these pulses, causing the molecules thereof to rotate. Due to the viscosity of the material, the molecules are not able to complete the rotation before a new pulse arrives and, therefore, a time-averaged voltage is observable for LC molecules; however, that pulse train produces certain fluctuation in the orientation of the LC molecules. This effect generates temporal fluctuations over the phase modulation on the order of a few milliseconds. Measurements performed with substantially higher integration times are less affected by the phase flicker. However, using a fast detector operating at high frequencies, the effect may alter the results and be harmful [52]. In addition, a reduction of the diffraction efficiency has been demonstrated when DOEs are encoded onto LCoS SLMs due to this effect [53].

On the other hand, when working with parallel-aligned devices there is a risk that a certain hysteresis will be generated in the molecules by applying a field continuously to them. For this reason, field inversion signals are generated, which alternate positive and negative values and which are added to the original pulse train. This effect happens both in analog and digital devices, adding an alternative source of flicker effect to them.

\section{II.3 - Characteristics of spatial light modulators}

Manufacturers are striving to improve the features of their devices continuously [54]. In general, a list of the most relevant characteristics of this type of modulators should include, among others, parameters like phase shift, 
spatial resolution, diffraction efficiency, refresh rate, reflectivity, flatness, and damage threshold.

The maximum phase shift indicates the maximum modulation that the SLM can perform over the 256 gray levels available on a common SLM (8-bit pixel values). It is advisable that the devices are capable of modulating at least a phase shift of $2 \pi$ to cover the entire range of phases. However, some devices are capable of modulating beyond that range. Please, see Fig. II.4. This could be useful to perform multiple tasks simultaneously with the modulator.

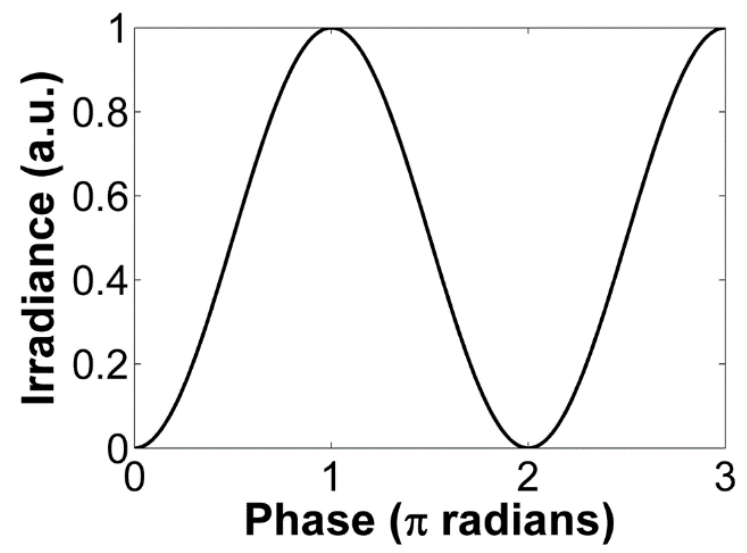

Fig. II.4 - Example of phase modulation. Measured intensity (in arbitrary units) is shown as a function of the phase. The measurement shows a phase shift of $3 \pi$ for the employed wavelength.

Spatial resolution and pixel size are two synonym terms. One could confuse spatial resolution with the number of pixels of the device (which is also usually known as resolution), nevertheless they are different terms. As SLMs are pixelated devices trying to encode continuous $2 \mathrm{D}$ patterns, what they are really doing is encoding an approximation of the desired pattern. By reducing the pixel size, this approximation would be more similar to the continuous pattern. The number of pixels multiplied by the pixel size results in the active area (or screen size). Obviously, it is possible to increase the active area of the screen by increasing the number of pixels. However, this only gives a larger region to work with, which can be useful in case of large beams. Nevertheless, the most common situation is to employ beam expanders to increase the beam width to fit the actual size of the SLM so, in principle, this is not a common problem. Reducing the pixel size while maintaining the active area would lead to an interesting increase in resolution. 
The response time is the average time that the SLM needs to change its phase from zero to $2 \pi$ (or vice versa). That is, the time it takes to make a phase change effective. Note that, a common protocol to measure this value consists in using a setup with the SLM placed between crossed polarizers. In this way, the SLM output can be obtained as intensity modulation. The response time is defined as the transition time from $10 \%$ to $90 \%$ of the final intensity. Therefore, this measure is only affected by the liquid crystal layer and not by other factors as the data transmission from the computer.

The frame rate indicates how many patterns is able to encode the SLM each second. Most common value is $60 \mathrm{~Hz}$. The refresh rate indicates how often the voltage applied over the liquid crystal layer is updated.

The flatness of the device is a fairly self-explanatory term. It is a measurement of the depth differences among the different parts of the surface of the SLM. Resistance is important where a high power laser beam is irradiating the SLM. It shows how much power by unit area is the SLM able to handle without physical damage or irreversible changes.

The fill factor is a measure of the size of the gaps between the mirrors or pixels of the device. The higher this value, the less gap between mirrors and the greater the amount of light reflected back to the system, directly influencing the modulator's efficiency. Reflectivity is the estimated percentage of light at the output of the modulator with respect to the input light.

\begin{tabular}{l|l|}
\hline \multicolumn{2}{|l|}{ PLUTO-2 Spatial Light Modulator - Microdisplay Features } \\
\hline Display Type: & Reflective LCOS (Phase Only) \\
\hline Resolution: & $1920 \times 1080$ \\
\hline Pixel Pitch: & $8.0 \mu \mathrm{m}$ \\
\hline Fill Factor: & $93 \%$ \\
\hline Active Area & $15.36 \times 8.64 \mathrm{~mm}$ (0.7" Diagonal) \\
\hline Addressing & $8 \mathrm{Bit}(256 \mathrm{Grey}$ Levels) \\
\hline Signal Formats & $\mathrm{DVI}-\mathrm{HDTV}$ Resolution \\
\hline Input Frame Rate & $60 \mathrm{~Hz}$ \\
\hline
\end{tabular}

Fig. II.5 - Characteristics of an SLM display. SLM Pluto - Holoeye. Obtained from [55]. 
Finally, diffraction efficiency is a very important keyword in the SLM field. It indicates how much light is affected by the action of the modulator. It is defined as the quotient of the first-order diffraction light level divided by the zero-order diffraction light level when a blazed grating is encoded into the screen of the SLM [56]. Several technical aspects play a role in this factor (fill factor, reflectivity...). All these characteristics as well as the problems discussed above are relevant when it comes to obtaining an optimized value of efficiency, and neglecting one of them would entail an important reduction of it.

But not only manufacturers are trying to increase that parameter by improving the related features, also researchers are developing technics that reduce the influence of these kind of harmful effects [52]. Among those efforts, one part focuses on making sure that there is a correct relation between the desired phase and the addressed value for that pixel. That is the goal of calibration of SLMs: adjust precisely the experimental response of the modulator to the theoretical curve that relates the addressed gray levels with the modulated phase.

\section{II.4 - Calibration of spatial light modulators}

Calibration of SLMs is almost a mandatory task when an SLM is employed within an optical system. Otherwise, discrepancies may appear between the desired phase pattern and the computer generated one. Usually manufacturers include look-up-tables (LUTS) to supply an initial curve function. However sometimes it is not provided, and even when they do, it may be not too precise due to several factors: first, the effect produced by the LC cell over the incident light depends on its wavelength. Secondly, the response of the modulator may change after a period, so it is advisable to re-calibrate the SLM from time to time or when a new light source with a different wavelength will be employed. 


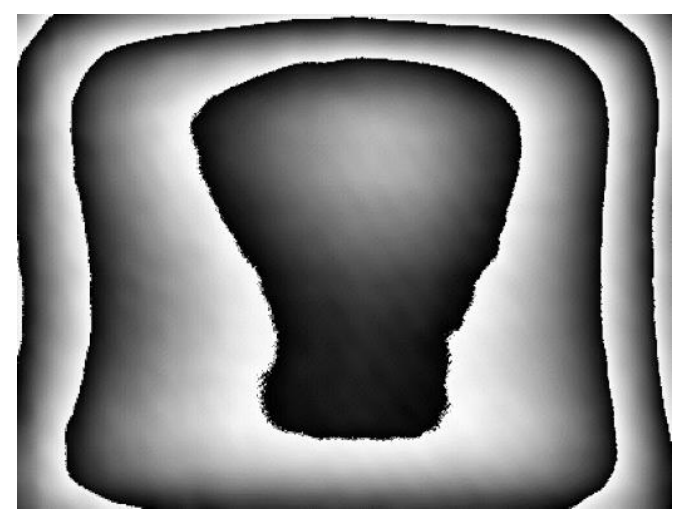

Fig. II.6 - Example of calibration mask. Hamamatsu. $840 \mathrm{~nm}$

Among the techniques proposed in the literature to calibrate this type of devices, two main groups can be distinguished: Interferometric-based methods and diffractive techniques.

The techniques included in the first group are characterized by performing the calibration generating interference patterns. These patterns are analyzed to obtain the phase response curve/calibration. Among other architectures, both Michelson [57] and Mach-Zehnder [58] interferometers have been successfully employed to carry out this task. Moreover, calibration techniques based on single-path interferometers have also been demonstrated as reliable methods to perform this task [59].

Methods based on diffraction usually encode DOEs that interact with light and generate a diffraction field. This field can be used to retrieve the phase shift that the light undergoes due to the action of the modulator. Sometimes these DOEs can be used just to measure one parameter (e.g. intensity) in the far field [60]. Finally, there are some techniques that exploits the capacity of the phaseonly SLM to be converted into an amplitude modulator by setting it between two crossed polarizers [61].

\section{Spatial calibration}

In general, techniques explained above are enough to properly calibrate an SLM, improving the efficiency of the modulator. In these techniques it is assumed that the phase response of a pixel is the same for each pixel of the SLM regardless of its position. However, some other methods suppose that local variations may appear. These variations are mainly attributed to differences in the thickness of the LC layer, and other causes such as local heating produced by the incident light or by inhomogeneities on the electric 
drive scheme [62]. Several works have been done to characterize the spatial response of these devices by defining space dependent LUTs. For instance in Engström et al. [62] a three-dimensional LUT is proposed, where the two first coordinates define the position of the pixel, while the last one is used for the desired phase. In Reichelt [58], a polynomial expression defines the behavior of the SLM depending on the position and the desired phase value. In this case, a polynomial evaluation must be carried out each time that a value is sent. This type of calibration is more complex and time-consuming, so it is advisable to do them only if necessary.

\section{II.5 - Diffraction-based phase calibration of SLM with binary phase Fresnel lenses}

We proposed a phase calibration diffractive technique. Interferometric techniques involve a very precise alignment and suffer from sensibility to vibrations. Moreover, the methods shown above require a large number of optical elements. In [58], polarizing beam splitters, linear polarizers and twolens Kepler type are included, apart from other common optical elements; while in [57] two polarizers, a beam splitter, a piezo mirror, a shutter, and a camera are required. On the other hand, diffractive-based methods may be affected by non-diffracted light, which introduces a harmful effect at the zero diffraction order and by residual intensity modulation. In our proposal just a single beam-splitter and an intensity detector is enough to get the calibration. As it does not use an interferometric arrangement, it is very insensitive to vibrations or other environmental fluctuations unlike two-arm interferometers.

The main idea of this technique lies in the encoding of a set of binary phase Fresnel lenses (BPFLS) into the phase-only SLM and measure the resulting irradiance foci with the detector. In this case, a charge-coupled device (CCD) camera is employed to measure the foci but another intensity-recording device (i.e. a bucket detector) can be used. A schematic of the setup is shown in Fig. II.7. 


\section{SLM}

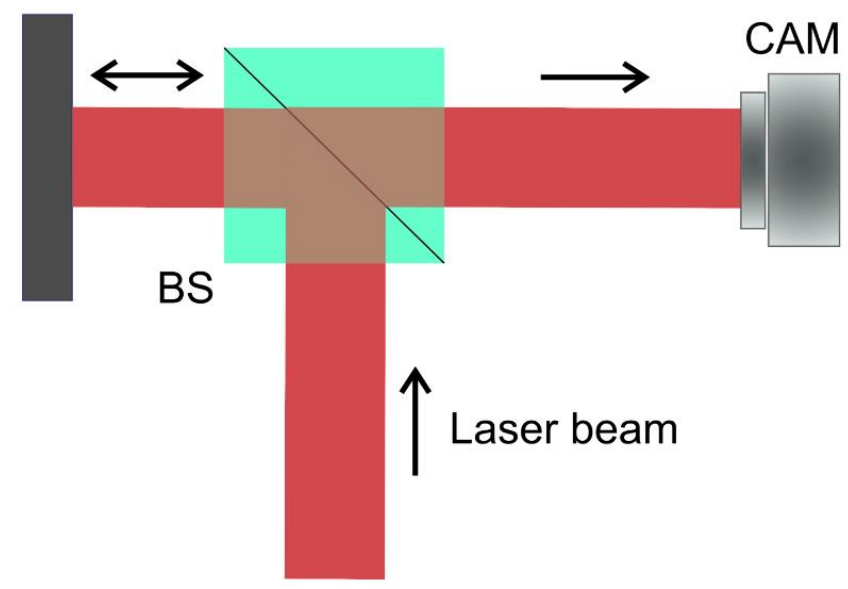

Fig. II.7-Experimental setup employed for phase calibration of the phase-only SLM. Only a camera and a beam-splitter cube are needed in addition to the SLM.

The BPFL is a lens made of a set of concentric rings that alternate their phase between two values $\left(\vartheta_{1}\right.$ and $\left.\vartheta_{2}\right)$. The diffraction efficiency $\eta_{m}$ can be expressed in mathematical terms as

$$
\eta_{m}=\left(\frac{2}{\pi m}\right)^{2} \sin ^{2}\left(\frac{\vartheta_{2}-\vartheta_{1}}{2}\right)
$$

where $m=1$ represents the selected focus. Each lens generates several foci located at different axial positions, and $m=1$ denotes the main focus or the one with the highest intensity. According to Eq. (I.1), it is evident that the diffraction efficiency of the lens depends on the difference $\vartheta_{2}-\vartheta_{1}$ between the two phase levels. 


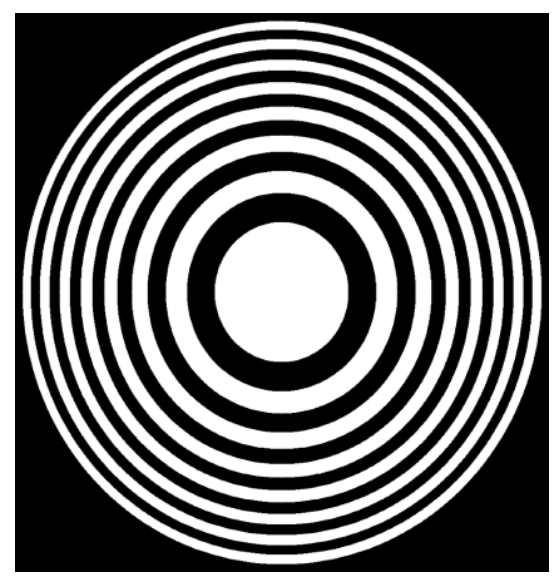

Fig. II.8-BPFL example. The concentric rings alternate their phase values between $\vartheta 1$ and $\vartheta 2$

To perform the calibration, two curves must be compared: an experimental one, which relates the level of gray sent to the device with the irradiance of the foci measured by the CCD, and another theoretical one, which relates that same irradiance to the phase, according to the Eq. (I.1). To obtain the experimental calibration curve, a series of steps, which are detailed below must be carried out. The objective is to obtain a curve that allows to know precisely $\vartheta_{2}$ in the generation of BPFL affects to the intensity of the measured focus.

The first step consists in the generation of the BPFLs that have to be sent to the modulator. In all of them, $\vartheta_{1}$ will take the value 0 , while $\vartheta_{2}$ will take a different value for each BPFL. To optimize the final result and obtain a calibration curve as accurate as possible, it is advisable to generate 255 BPFLs from 1 to 255; however, a faster calibration can be obtained by generating a reduced number of lenses. In this way, fewer points will be enough to obtain the gray level - radiance curve and less time is needed to make the measurements.

The second step is to synchronize the modulator with the camera (or the device used to measure the irradiance). Then, it is possible to send the BPFLs generated in the previous step to the modulator. As the spot widths remains the same for all BPFLs, the criteria used to measure diffraction efficiency is unique for all measurements. In our case, the $1 / \mathrm{e}^{2}$ criterion was employed to define the beam width. 
Once the experimental curve has already been achieved (Fig. II.9.a), the theoretical one can be obtained from Eq. (I.1). This curve is represented in Fig. II.9.b. The main difference between both figures is that the first relates the irradiance and the gray level while the second one links the irradiance with the phase. The comparison between both curves allows the correlation of the gray levels with the phase. To fit both curves, it must be taken into account that for each gray level sent to the SLM, a phase value associated to that gray level is encoded at the LC layer. In that sense, and thanks to the theoretical basis explained before, the point of maximum irradiance can be related to a phase difference between $\vartheta_{1}$ and $\vartheta_{2}$ of $\pi$, and the minimum with a phase difference of 0 or a multiple of $2 \pi$. Please, note that depending on the modulator model, the phase shift that the modulator can encode may be greater than $2 \pi$. One should also keep in mind that the experimental curve should be normalized to improve the result as the irradiance values are measured in arbitrary units.

(a)

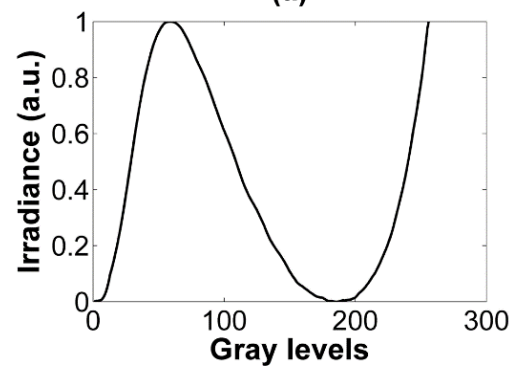

(b)

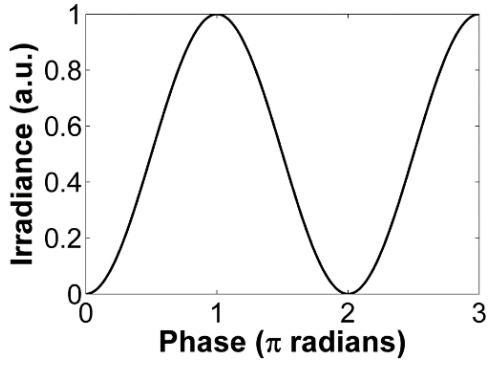

(c)

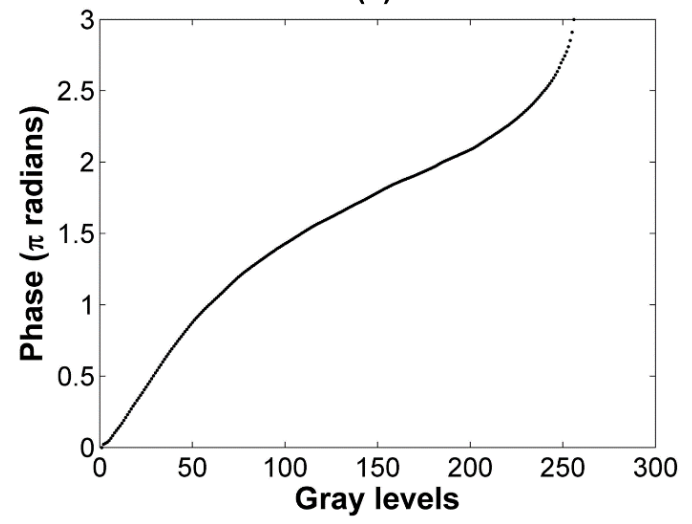

Fig. II.9 - Experimental results. a) experimental curve obtained by sending 255 BPFLs. b) theoretical curve obtained. c) calibration curve obtained fitting the gray levels and the phase values. 
The next step consists of separating each of the minimum-maximum or maximum-minimum sections, in the experimental curve, as they are related to phase shifts of $\pi$ (from 0 to $\pi$, from $\pi$ to $2 \pi$, and so on). The irradiance values of those sections are substituted into Eq. (I.1) linking the gray level used to obtain a certain irradiance value with the phase. The reason to split in sections is because a real function must be obtained. At this point, the resulting function of joining the different sections accurately indicates the phase encoded in the modulator according to the level of gray sent to it, which is precisely the curve that we want to obtain as a result of the calibration, and can be employed as a LUT function (Fig. II.9.c). From Fig. II.9.b one can realize that the phase-only SLM calibrated here is able to encode a phase shift of $3 \pi$.

To test the validity of the presented method, a comparison (Fig. II.10) with one of the best-known techniques [63] has been made. In this technique, 255 Ronchi grating patterns, designed in a similar way to the BPFLs, are sent to the modulator; the setup is almost identical to the one employed in our experiment but including an extra refractive lens. The irradiance is also measured analogously to our experiment, using the same $1 / \mathrm{e}^{2}$ criterion.

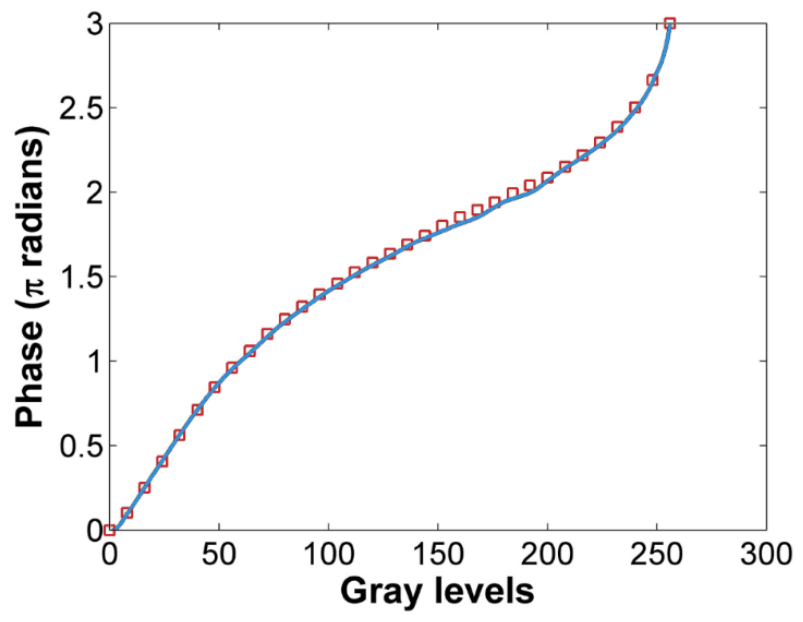

Fig. II.10 - Comparison between the proposed technique using BPFLS (red dots) with a well-stablished calibration method using Ronchi gratings (blue line).

At a glance, one can realize that both results agree satisfactorily. In detail, the calculated root mean squared error between the two methods is approximately $6 \%$. Differences are mainly produced due to laser fluctuations 
in both position and energy. These results provide a clear proof that the method offers reliable data for phase calibration purposes.

In order to prove the utility of the technique in other experimental conditions, other tests has been performed with different specifications: further periods for the BPFLs, a different criterion for the beam width (instead of the $1 / \mathrm{e}^{2}$ ), and selecting a different focal plane $(m \neq 1)$ for the focal measurements. The results obtained, in all cases, yield root mean squared errors less than $5 \%$. Moreover, to predict how different amplitude distributions affects the technique, simulations have been made. The result of the simulations confirms that this parameter does not influence the calibrations function, which is something expected as relative irradiance measurements are performed.

This method has some restrictions: the pixel size of the SLM limits the minimum size of the rings that make up the BPFLs. This prevents the generation of a main focus very close to the SLM, since rings smaller than the pixel size of the SLM are needed. This technique requires at least two pixels to properly encode a ring, leading to a closest distance of, approximately, 150 $\mathrm{mm}$. On the other hand, in this technique all the pixels of the modulator are involved in the calibration. That means that the LUT obtained is global and is used equally in all pixels, regardless of their spatial position on the screen; however, as discussed before, it is possible that not all pixels respond in the same way. For most applications and devices this is not a problem, since the decrease in diffraction efficiency is not significant. More information about the results obtained with this experiment can be found in [64]. 


\section{Chapter III - Beam shaping using phase-only SLMs}

The action of modifying the amplitude and phase of the complex field that describes the electromagnetic wave nature of laser beams is known as beam shaping. For some applications it is usually enough to take care of the irradiance distribution at a certain plane. In these cases, the effects of the phase can be disregarded, simplifying in that way the beam shaping process. In this chapter most relevant techniques used to spatially manipulate the amplitude of a laser beam with phase-only SLMs are summarized. After that, an own novel method to do that is described. It allows generating arbitrary three-dimensional distributions of foci with high accuracy setting over fundamental laser beam parameters e. g., focal positions, peak energy, etc. Such energy distributions were tested as useful illumination patterns to investigate multifilamentation processes in fused silica.

\section{III.1 - Conventional encoding techniques}

\section{Computer-generated holography}

Thanks to holography, it is possible to record and later reconstruct a certain irradiance distribution or even the complex field of a light beam by the interference of two coherent light beams. One of the biggest advantages of holography is that it is capable of capturing the 3D geometry of a scene and reconstruct it as a $3 \mathrm{D}$ image. For this reason, it has become one of the most used techniques when desired distributions of light are requested.

Computer-generated holography is a technique based on holography. Instead of optically capturing the hologram, a computer is used to numerically calculate it. The calculated hologram can be encoded in a digital device to reconstruct the desired distribution. Therefore, the idea is quite easy: define a target complex field and then apply the two-dimensional Fourier transform. Nevertheless, the full complex field is required to perform this task because the two-dimensional Fourier transform is complex. To overcome above limitation several encoding methods have been proposed. Among those techniques, some of them perform transformations before the generation of the hologram. The idea is to encode the original pattern with only one phase element. This is the case, for instance, of a technique known as one step phase retrieval [65]. It is based on the addition of a phase term to the source image before the generation of the hologram to obtain a hologram formed by a single phase term. Similarly, the sampled phase-only hologram method [66], employs 
a lattice to down-sample an image to obtain a hologram with uniform amplitude. Therefore, only the phase component contains information about the image and can be directly encoded into a phase-only SLM. Although these methods offer acceptable results, the transformations performed to the original image make them not optimal, but an approximation. Something similar occurs with one of the classic methods to solve this problem: iterative Fourier transform algorithms (IFTA).

\section{Iterative Fourier transform algorithms}

Beam propagation is affected by the phase modulation of the SLM, since the interference of the different parts of the beam influences the intensity profile at a certain distance. The main issue is that only one phase modulation is not enough to obtain an exact beam shaping. So iterative algorithms employ inverse Fourier transforms to calculate the best phase pattern at the SLM that reproduces the desired target intensity at the sample plane.

Among the most relevant algorithms for beam shaping are the classical Gerchberg-Saxton [18] and Yang-Gu [19] algorithms. The GSA and similar ones work in an iterative procedure; that is, they start with a certain phase $\varphi_{0}$, which is Fourier transformed to get a complex field $\left|A_{0}\right| e^{i \theta_{0}}$. The amplitude $\left|A_{0}\right|$ is compared with the target amplitude $|B|$. If the difference between both is less than the defined error, the algorithm ends. Otherwise, $\left|A_{0}\right|$ is replaced by $|B|$, getting the complex field $|B| e^{i \theta_{0}}$. This result is inversely transformed, obtaining the complex field $\left|a_{1}\right| e^{i \varphi_{1}}$. Then, the input beam function $|b|$ is imposed at the result, resulting in $|b| e^{i \varphi_{1}}$. At that point, the algorithm repeats the steps already discussed. This loop is repeated $n$ times, until the algorithm converges or the maximum number of iterations $N$ defined by the user is reached. This limit prevents the algorithm to be blocked when convergence is not reached, possibly because the algorithm tends to fall in local minimums or due to a too strict convergence threshold. Another classical iterative algorithm is known as direct binary search [67]. This approach employs an IFTA-based algorithm to minimize the mean squared error between a binary hologram and the reconstructed image.

The GSA was originated in the 70's and has some drawbacks that have led researchers to develop improved versions of it. One of the biggest disadvantages of this technique is precisely that convergence is not guaranteed [68]. One way to prevent the algorithm from falling into a local minimum lies in varying the starting point since the result depends, to a large extent, on the initial phase $\varphi_{0}$. In fact, the most advisable thing is to try to 
approximate that value as much as possible to the final value. This is only possible from an approximate solution or from a solution to a similar problem. Otherwise, an estimation or insertion of a random value should be carried out. Another drawback of this technique is that, in principle, only the desired intensity can be defined at the output plane and, therefore, the obtained phase is random. This deficit of phase control generates speckle noise due to the destructive interaction of contiguous patterns with different phases, forming high contrast regions and degrading the quality of the projected pattern. This is negative for most applications but is especially harmful in certain cases such as multiphoton microscopy, where two-photon excitation depends on the square of the light intensity and, therefore, these irregularities may cause image blurring.

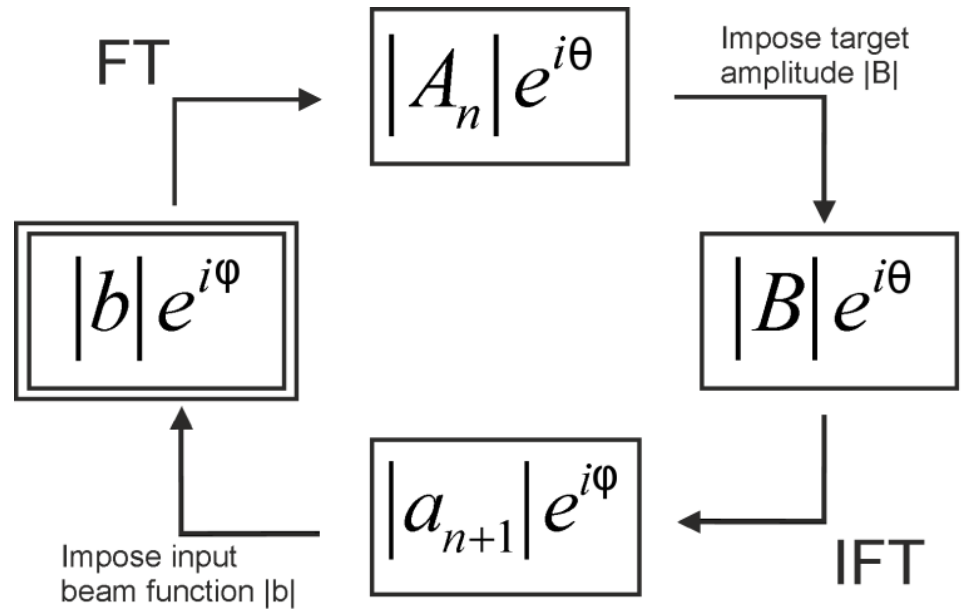

Fig. III.1 - Schematic of an IFTA. The squares represent the states while the arrows mean transitions. The double-lined state is the starting point.

Another problem generally associated with the GSA is its inability to generate multiple intensity levels within the different parts of the pattern due to the impossibility of manipulating the amount of energy put into them. Due to the above-mentioned drawbacks, numerous improvements have been made to the original algorithm. For example, the Yang-Gu algorithm is a generalization of the GSA. While GSA is able to solve the amplitude-phase retrieval problem using linear unitary transform systems. Yang-Gu includes a non-unitary optical system remaining identical for a unitary transform system. Hence, the former algorithm is able to converge in cases in which the GSA is not capable, and is more stable in the presence of random noise [19]. 
Other examples include the weighted Gerchberg-Saxton (WGS) [69], which introduces a new parameter referred as weight, that allows to reduce the amplitude deviations from the average amplitude of the electric field. Additionally, some techniques have been developed to add phase control to WGS, resulting in an algorithm known as weighted Gerchberg-Saxton with phase control (WGS-PC) [70]. This algorithm has been probed to simultaneously control both the phase and amplitude of the generated patterns with almost perfect uniformity over the amplitude of the field. Previous algorithms typically constraint only the amplitude whereas WGS-PC also constraints the phase of the projected pattern. The main limitation of this technique lies in the ability of generate only 1-D curved patterns dropping dramatically its efficiency when 2-D patterns are employed. In addition, techniques able of generating multiple energy levels have been reported [71]. By adding a weighting parameter to define an objective intensity to the GS algorithm, it is possible to obtain a hologram with different relative intensities that allows, for example, generating a uniform light distribution on the irradiance pattern regardless of the distribution of the light source or the position-dependent diffraction efficiency of the LC-SLM. Finally, it is possible to independently manipulate multiple incoherent light beams with different wavelength by using a single phase element [72]. In this method, each $2 \pi$ hologram is calculated separately and, by using an SLM with a high modulation range $(\approx 10 \pi)$, these holograms are combined on a multi-level hologram. In that way, the final multi-level hologram reconstructs the original patterns at preselected wavelengths, at the cost of losing diffraction efficiency.

\section{Generalized-phase contrast}

To measure the intensity of light it is enough to use wavelength sensitive detectors that are capable of quantifying the amount of energy received. A CCD sensor would be an example of one of these devices. However, measuring the spatial phase of an incident light beam is more complicated. CCD detectors are not sensitive to phase variations so it is necessary to employ techniques that generate phase-dependent intensity variations. For example, by using interferometric methods to measure unknown phase distributions [73]. The Zernike phase contrast technique is a method to measure phase perturbations based on the use of a Fourier plane phase-shifting filter.

Generalized phase contrast is the generalization of the Zernike's method. It is a similar technique, also based on interferometry, but not limited by the operational constraints of Zernike's method which provides an extended range of phase contrast [20]. In short, GPC generates an amplitude pattern that is 
sensitive to the phase distortions given at the input plane of an optical imaging system. Hence, it can be used to measure these phase distortions by recording irradiance patterns at the output plane. Also, for beam shaping purposes: instead of measuring a phase from an irradiance pattern, encoding a phase mask on a spatial light modulator will produce an arbitrary intensity distribution at the output plane. In particular, an on-axis spatial filtering operation in the Fourier plane of a $4 \mathrm{f}$ imaging system is employed to convert a phase disturbance at the input of the system into an irradiance distribution at the output plane.

Laser beam

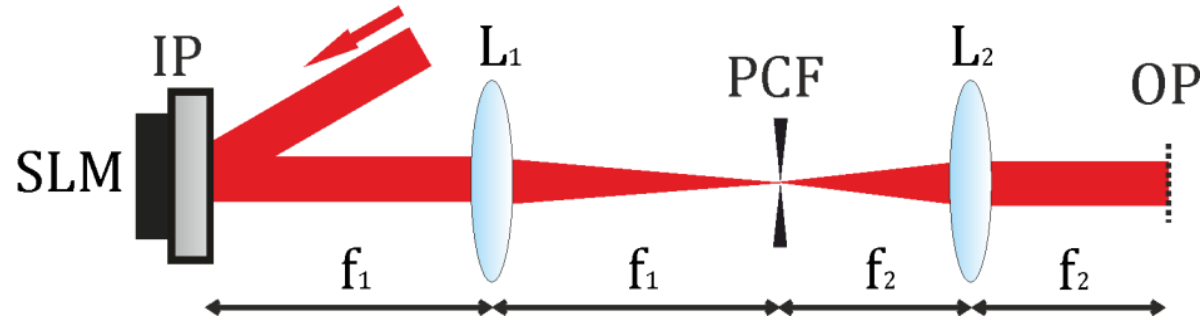

Fig. III.2 - Typical GPC setup. At the input plane (IP) an SLM is placed encoding

an input phase mask. L1 and L2 form a 4 f imaging system. At the output plane (OP) an intensity pattern formed by the interference of the nondiffracted light and the image of the object placed at the IP.

A typical system used in this method is shown in Fig. III.2. Basically two lenses $\left(L_{1}\right.$ and $\left.L_{2}\right)$ form a $4 f$ optical system. At the input plane of the system, an SLM is placed. At the Fourier plane, a phase contrast filter (PCF) is introduced. The PCF introduces a phase shift within a radius located in its central part, while in the rest of its surface it allows the passage of light without being modified. The phase mask encoded into the SLM is employed to control the distribution of light among the surface of the PCF. Light is focused by the first lens $\left(L_{1}\right)$ at the on-axis (central) region of the PCF is considered as focused light. Light distributed along the rest of the surface of the filter is considered as scattered light. The focused light acts as the reference wave of a common-path interferometer (CPI), coherently mixed with the scattered light to generate the arbitrary amplitude distribution at the output plane of the imaging system [74].

Unlike the aforementioned CGHs, where each point of the hologram receives contributions from the entire input pattern, here the output image is formed by point-to-point mapping, commonly reducing the efficiency of the method. 
This technique is suitable for CPI due to the capacity of this type of systems to increase tolerance to vibrations and air turbulences with respect to systems in which both arms travel in different ways. From a general point of view, GPC has several applications besides synthesizing gray-level light patterns [75], including the generation of multiple optical tweezers [76] or optical security [77].

\section{III.2 - Shaping techniques for focusing diffractive elements}

Another way to obtain arbitrary irradiance patterns is by using a specifically designed DOE. In particular, Davis et al. [78] used an SLM to encode a grating with variable depth. In that way, they are able to control the diffraction efficiency of the grating, allowing for the amplitude modulation of either the first order or the zero order. While this kind of methods successfully modulate the amplitude of a light beam, the diffraction efficiency is low because they require to send a large part of the light to the remaining diffraction order.

Among DOEs, focusing diffractive elements (FDEs) can be considered as simple Fresnel holograms that generate controlled irradiance spots at a certain distance from the reconstruction plane. Encoding two-dimensional and threedimensional arrays of FDEs in a single Fresnel hologram is interesting because it allows to generate complex irradiance distributions useful for multiple applications, including formation of Bessel beam arrays [79] or femtosecond laser processing [80]. Also parallel generation of non-linear effects in both the axial [27] and transversal direction [81] enables the application of this type of techniques to fields such as multiphoton microscopy [82] or filamentation [83]. A wide number of encoding methods has been reported recently to generate the appropriate set of parameters for each application. For instance, in [80] a set of Fresnel lenses is spatially multiplexed onto a single DOE to perform three-dimensional laser processing. In this manuscript, an algorithm aimed to equalize the intensities of all spots is included due to the relevance of this parameter for material processing applications. The algorithm is dedicated to finding the optimal combination of central focal points for a set of lenses. Another approach implements shifted phase vortices to generate arrays of diffraction-limited non-Airy spots [84]. Similar techniques have demonstrated their usefulness for the formation of multifocal arrays of non-diffractive beams. For instance in [79], a hologram made up of multiple overlapped axicons is employed to generate an array of Bessel beams. In [82], a twodimensional array of foci is formed by the combination of a microscope objective and a pre-calculated phase pattern encoded into an SLM. The SLM is 
placed at the conjugated plane of the back aperture of the microscope objective. This method allows increasing the scan speed of the microscope by using multiple foci simultaneously. In addition, the combination of similar strategies with axial-confinement methods has been reported [85], demonstrating the ability of the method to temporarily separate the spots.

\section{III.3 - Diffractive control of 3D multifilamentation}

In 2016, we presented a work where the ability to encode diffractive phase elements (DPEs) capable of generating three-dimensional filament distributions with separations of the order of a few micrometers was demonstrated [86]. Filamentation is a phenomenon by which a beam is able to propagate a distance greater than the Rayleigh distance associated with that pulse. Two main processes influence this phenomenon, first the plasma tends to generate a defocus in the beam, and on the other hand, the Kerr effect tends to focus it. Generation of multiple filaments in a fused silica crystal has been investigated in several articles $[87,88]$ in which arrays of filaments are generated using arrays of lenses; however, these systems may not be the most suitable for certain applications for several reasons. First, it is not capable of generating filaments in certain positions. For example, the distance between two filaments cannot be less than the sum of their radii. It is possible to use additional optics to solve this issue, but that implies using an unnecessarily complex setup. Secondly, the energy of each filament depends directly on the distribution of the beam energy in the plane of the lens. This is because each filament is generated by a lens located in a different spatial position. Lastly, the implementation of the lens array causes a reduction of the numerical aperture due to the reduction of the lens diameter.

In this contribution, a diffractive technique capable of generating threedimensional filament distributions, which does not suffer from the aforementioned problems, has been proposed. A DPE, that contains the information of a set of spatially multiplexed diffractive lenses, is generated and encoded in a phase-only SLM. A femtosecond laser impinges the SLM and the diffracted light is focused inside a fused silica. In that way, each encoded lens generates a filament inside the fused silica with a control over its position of the order of a few micrometers. To obtain similar irradiance levels for each filament, all lenses are spatially multiplexed and distributed uniformly along the SLM surface. In that way, it is possible to obtain similar amounts of energy at the focal points of the encoded Fresnel lenses even if the laser irradiance is not uniform at the SLM plane. 
Other techniques based on spatially multiplexed Fresnel lenses have been reported previously. For example, in $[89,90]$ the encoded pattern is generated by the random sampling of several lenses. The lenses are created in advance, and an algorithm is responsible for assign randomly each pixel (or group of pixels) to a different lens. Energy can be controlled by increasing or reducing the number of pixels employed for each lens. A similar approach been reported in [91]. In this case, the final result is optimized by applying the WGS algorithm. However, these methods are based on iterative algorithms that obtains an approximate solution rather than the ideal solution or suffer a quadratic reduction of diffraction efficiency by increasing the number of lenses.

In our proposal, to encode the phase element in the SLM, it is necessary to calculate $\mathrm{N}$ off-axis lenses and $\mathrm{N}$ complementary binary masks $M_{n}(x, y)$, where $x$ and $y$ represent the coordinates of a Cartesian coordinate system, that meet the condition $\sum_{n=1}^{N} M_{n}(x, y)=1$, where $n=1 \ldots N$ represents each lens. These masks are used to sample the different lenses, defined by $\varphi_{n}=\bmod _{2 \pi}\left\{\pi\left[\left(x-x_{0}\right)^{2}+\left(y-y_{0}\right)^{2}\right] /\left(f_{n} \lambda_{0}\right)\right\}$, in such a way that pixels that encode each lens do not overlap on the spatial domain. The function $\bmod _{2 \pi}$ yields modulo $2 \pi, f_{n}$ is the focal length for the $n$th lens, and $\lambda_{0}$ is the central wavelength of the employed light beam. Finally, $x-x_{0}$ and $y-y_{0}$ represent the arbitrary displacement of the center of the lens that produces a dependent displacement of the focused light that generates the filament.

Multiplying the lenses $\varphi_{n}$ by the masks $M_{n}$, that we must remember that do not overlap, we multiplex them spatially. In this way, the DPE contains the information corresponding to all the lenses. Therefore, the DPE is the result of sampling the binary masks to the different lenses and adding the result, as is shown in expression (II.1)

$$
\operatorname{DPE}(x, y)=\sum_{n=1}^{N} \varphi_{n}(x, y) * M_{n}(x, y)
$$

To define the masks $M_{n}$, the easiest way is to divide the surface of the modulator in superpixels formed by $m * m=N$ pixels. For example to generate $N=4$ filaments, each superpixel would be made up of $2 * 2$ pixel cells. (See Fig. III.3.a). It is not necessary $N$ to be power of another number, it is possible to generate any number of filaments using asymmetric masks; however, for this example it is easier to represent it with a number $N$ power 
of $m$. Then, once the surface of the modulator is divided into super pixels, it is time to generate the different masks $M_{n}$. For example, in Fig. III.3.b, mask $M_{1}$ is shown, all pixels corresponding to the first row and the first column of each super pixel has been assigned the value 1, while all other pixels remain with the value 0 .
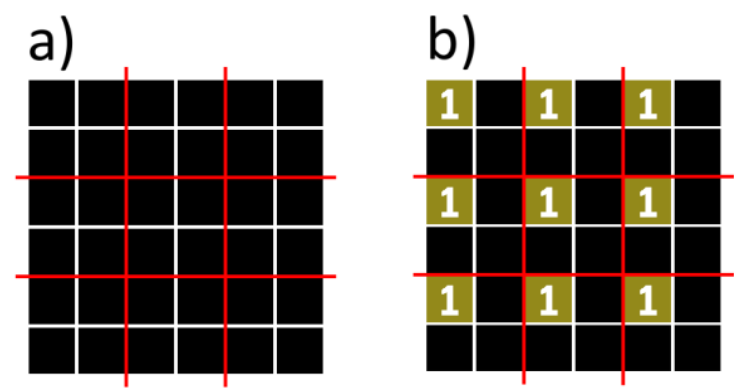

Fig. III.3 - a) Example of a mask formed by $2 * 2$ superpixels. b) Pixels located in the first row and first column of each superpixel are set to 1.

Each other mask $\left(M_{2}, M_{3}\right.$, and $\left.M_{4}\right)$ is generated analogously, using one of the other pixels of each super pixel for each one of these masks. For example for $M_{2}$, the pixel set to 1 can be the one corresponding to the first row but second column in this case. Then, each lens is multiplied by its corresponding mask; that is: $\varphi_{1} * M_{1}, \varphi_{2} * M_{2}$ and so on. Since the value in the masks is always 1 or 0 , the value in the auxiliary pattern will be either 0 (when the value in the mask is 0 ) or the same value as in the phase element $\varphi_{n}$ (when the value in the mask is 1 ). The result in the example will be four auxiliary patterns shown in Fig. III.4.

a)

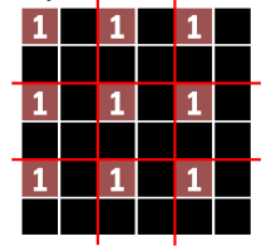

b)

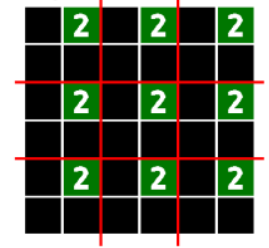

c)

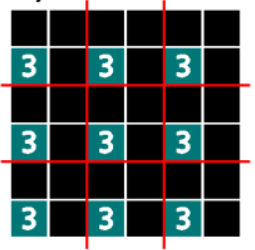

d)

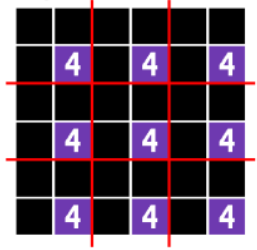

Fig. III.4 - a) Example of the four auxiliary patterns generated for a $2 * 2$ superpixel arrangement.

a), b), c), and d) represent the result of $\varphi_{1} * M_{1}, \varphi_{2} * M_{2}, \varphi_{3} * M_{3}$, and $\varphi_{4}$

* $\mathrm{M}_{4}$ respectively. The values 1, 2, 3, and 4 inside the matrices represent that this phase value comes from $\varphi_{1}, \varphi_{2}, \varphi_{3}$, and $\varphi_{4}$ respectively. 
Pixels shown in black contain the value 0 , while colored pixels now contain the phase value of the corresponding lens. For example, in Fig. III.4.a each pixel represented as 1 does not necessarily contain the value 1 , but rather the value contained in $\varphi_{1}(x, y)$, where $x$ and $y$ indicate the position of that pixel into the matrix.

The last step to obtain the final DPE is to sum the auxiliary patterns between them, as indicated in the Eq. (II.1). As can be seen in the example shown in Fig. III.4.a, each pixel only contains phase information of one of the lenses and, therefore, it is obvious that each Fresnel lens is encoded by a different set of pixels of the SLM. This means that the control of the different parameters of each lens (position, focal distance, efficiency, etc.) can be carried out independently.

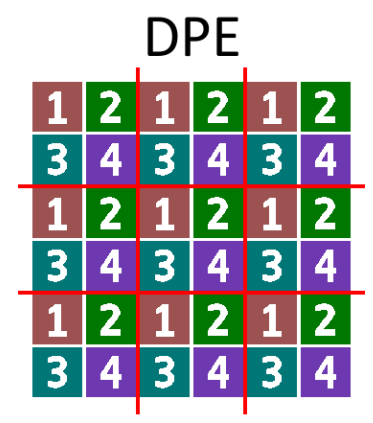

Fig. III.5 - Final DPE obtained from the sum of the auxiliary patterns shown in Fig. III.4.

The number of lenses that can be encoded in a single DPE is strongly related to the pixel size of the SLM. This is because the $\varphi(x, y)$ lens is calculated as a continuous function but when it is encoded into the modulator, it is discretized. Therefore, the smaller the pixel size, the smaller the error and the greater the efficiency of the lens and the energy at the focal point. On the other hand, the more lenses encoded in a single DPE, the fewer pixels are used to encode it and, therefore, the lower the efficiency of it. In this case, with a pixel size of $8 \mu \mathrm{m}$, up to nine lenses can be encoded simultaneously. Note that to generate the non-linear effect present in the filamentation process it is necessary to reach a certain energy threshold.

Several experiments were performed to show the potential of the developed method. First, the ability to control at will various parameters such as the position and number of filaments, distribution of energy, etc. was demonstrated. Secondly, the system was used to study the mutual coherence 
between filaments developed in fused silica. In these experiments, the optical system used is shown in Fig. III.6. It basically consists of a $4 f$ imaging system, composed by a couple of lenses with focal lengths $f_{1}=300 \mathrm{~mm}$ and $f_{2}=$ $150 \mathrm{~mm}$, in which an SLM has been placed on the input plane. To send properly the incident beam emitted by a Ti:Sapphire femtosecond laser to the SLM, the beam is appropriately enlarged using beam expanders to fit the whole SLM surface. Moreover, to impinge the SLM with normal incidence, a pellicle beam splitter is placed so that it reflects light towards the SLM, but allowing the passage of the reflected light. In the SLM, the DPEs described above are projected. An image of those DPEs is formed in the output plane of the $4 f$ system. This allows us to get access to positions near the DPE. Finally, a fused silica sample is placed at the central focal distance of the different lenses encoded into the DPE in such a way that all the filaments are generated inside the sample. Moreover, the DPE has been generated with specific focal lengths allowing all the filaments to remain inside the fused silica. Nondiffracted light does not focus by the lenses and, therefore, does not contribute to the filament formation. To record the images used in these experiments, two CCD cameras are located on one side and behind the fused silica. By using different optical elements (lenses, microscope objectives), images of the filaments are formed onto the CCD cameras. The first one was placed beside the fused silica, parallel to the propagation axis of the filaments, and it was used to record the images of the filaments inside the sample. The second camera was placed after the rear face of the fused silica, perpendicular to the propagation axis, and it was employed to record images originated by the interference of several filaments. The magnification of these images has been adjusted to cover the entire surface of the CCD.

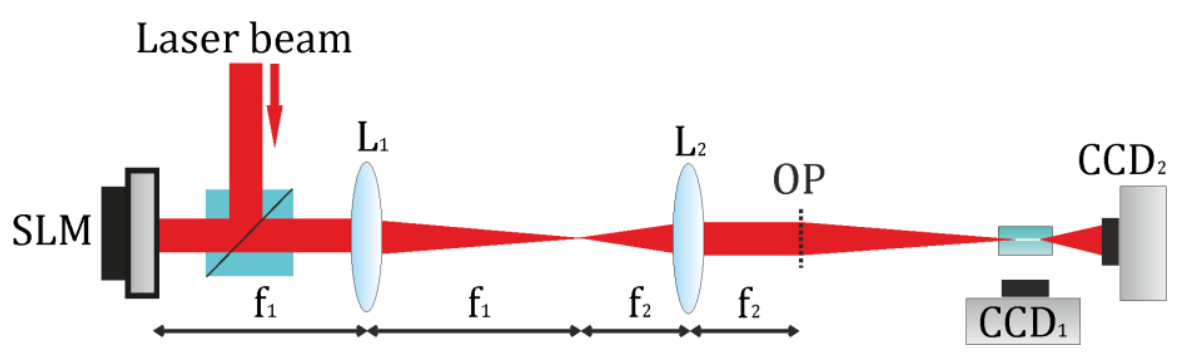

Fig. III.6-Optical setup employed for the generation of multiple filaments. $\mathrm{L}_{1}$ and $\mathrm{L}_{2}$ : lenses. $\mathrm{f}_{1}$ and $\mathrm{f}_{2}$ : focal distances of $\mathrm{L}_{1}$ and $\mathrm{L}_{2}$. OP: Output plane of the 4f imaging system. FS: Fused silica.

The objective of the first experiment is to demonstrate the validity of the method to generate multiple filaments in fused silica with arbitrary spatial 
distribution and controlled energy. Specifically, experiments that demonstrate the ability to generate different filament distributions, varying both the position and the number of filaments and the ability to equalize the amount of energy of each filament independently have been carried out.

Fig. III.7 shows four different spatial distributions of filaments. Each distribution has been achieved using a different DPE, encoded in the SLM as mentioned above. These DPEs focus the light inside the fused silica generating the non-linear effect that gives rise to the filament. Fig. III.7.a shows the starting point: nine filaments separated $128 \mu \mathrm{m}$ between them are generated by a DPE composed of nine converging lenses with the same focal length $f=$ $245 \mathrm{~mm}$. To test the ability of the method to modify the number of filaments generated, a distribution of five filaments is shown in Fig. III.7.b. In this case, when generating the DPE, four of the nine convergent lenses have been changed by divergent ones $(f=-500 \mathrm{~mm})$. In this way, the energy associated with these lenses can be neglected since it is far from reaching the threshold necessary to generate non-linear effects inside the fused silica. Similarly, in Fig. III.7.c and 3.9.d, different distributions have been generated by changing convergent lenses for divergent ones. Specifically in Fig. III.7.c it is shown that the distance between filaments can be different. Separation between the upper and the nearest filament is $384 \mu \mathrm{m}$, while the separation between the central filament and the nearest ones is $128 \mu \mathrm{m}$. In Fig. III.7.d it is shown that the distribution does not have to be symmetric.
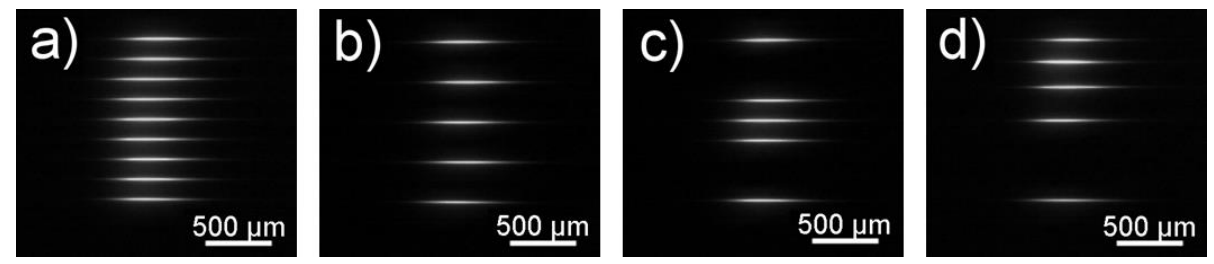

Fig. III.7-Recorded images corresponding to the first experiment: four different distributions of filaments generated in fused silica

In all four images, filaments are in almost the same axial positions. Variances are in the order of the pixel size of the employed SLM $(8 \mu \mathrm{m})$. Really to obtain this result it is necessary to adjust the focal lengths of the different lenses that make up the DPE. Due to several factors including a slightly different optical path and small misalignments of the beam onto the SLM plane, each filament is generated in a different axial position if all the lenses are encoded with the same focal length. To correct it and obtain the desired result, the focal distances are modified experimentally in real time, achieving a uniform result. 
Something similar happens with energies. In principle, all lenses should be equally efficient; however, due to the experimental conditions it is possible to find that some filaments are more intense than others are. The encoding technique allows to control the energy of each filament independently, thus achieving that the energy variances on the filaments of Fig. III.7 are smaller than $8 \%$. For this purpose, the diffraction efficiency of each lens can be reduced to obtain a similar amount of energy for each filament by introducing a new parameter $\alpha$. This parameter, which ranges from zero to one, modulates the quadratic phase $\left(\varphi_{n}\right)$ of the lens when multiplied by it. In that way, when $\alpha$ takes the value one, the lens remains unchanged but when it takes a lower value, the lens becomes less efficient generating a less energetic filament.

In addition, should be mentioned that the broadband spectral illumination employed $(760 \mathrm{~nm}-840 \mathrm{~nm}$ ) significantly influences the results obtained. Diffractive lenses focuses each wavelength of the ultrashort pulse at a different axial position. All lenses used here are optimized for the central wavelength $(800 \mathrm{~nm})$, but only for that wavelength. In the temporal domain, due to the difference of propagation time between pulses coming from the different parts of the lens, an increase in the temporal duration of the pulse is expected. On the other hand, the same thing should not happen using refractive lenses, where the Rayleigh range is supposed to be shorter.
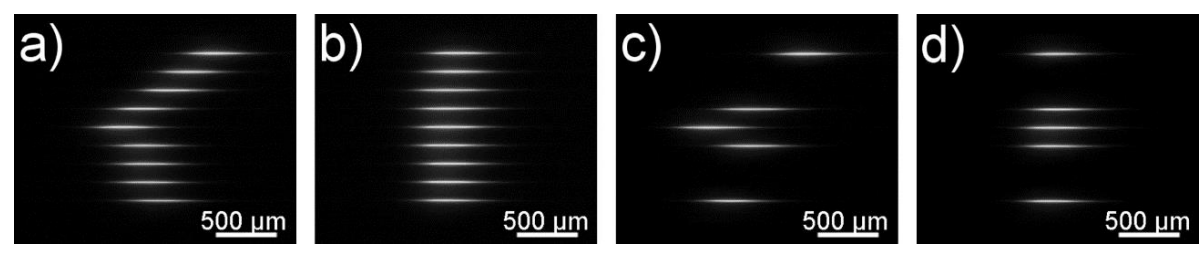

Fig. III.8 - Images of filaments demonstrating the correction ability of the encoding technique. a) and c) images without axial position correction. b) and d) images with position correction.

Fig. III.8.a and Fig. III.8.c show two sets of nine and five filaments respectively without correction. In both cases, the focal length of all the lenses that make up the DPEs used to generate these filaments is the same $(f=245 \mathrm{~mm})$. To obtain the images 3.10.b and 3.10.d, the focal lengths of each lens have been modified individually to correct it and to harmonize the axial positions of the filaments. In particular for Fig. III.8.d, the focal lengths used are, from top to 
bottom: $f_{1}=243.7 \mathrm{~mm}, f_{2}=245.0 \mathrm{~mm}, f_{3}=246.0 \mathrm{~mm}, f_{4}=245.0 \mathrm{~mm}$, and $f_{5}=245.6 \mathrm{~mm}$.

Once the ability of the method to generate a given number of filaments with arbitrary energies and positions has been demonstrated, the optical system has been used to investigate the mutual coherence between filaments in fused silica. When two or more filaments are close enough, interference effects may appear between them. In the next experiment, and due to the ability of the method to place the filaments in the desired position with pixel-size precision, the effect of different filament distributions over the interference pattern has been studied.

The optical setup used in this experiment is the same as in the previous one (shown in Fig. III.6) but adding a bandpass filter suited to allow the passage of light from the non-linear generation and block non-converted infrared light. The two cameras located next to and behind the fused silica sample allow recording images of the filaments and the interference pattern simultaneously.

Fig. III.9 shows the interference pattern generated by two filaments for four different lateral separations: $80 \mu \mathrm{m}, 128 \mu \mathrm{m}, 256 \mu \mathrm{m}$, and $512 \mu \mathrm{m}$. In the upper part of the figure, the filament distributions within the fused silica recorded by the lateral CCD are shown. In the upper right corner of each image, separation $I$ between the filaments displayed in that image is shown. In the bottom part of the figure, the interference patterns generated by the corresponding filament distributions are shown. These patterns correspond to the typical longitudinal fringe patterns. In addition, the period of these fringes is related to the distance between filaments, as expected: the longer the separation, the shorter the period and vice-versa.

Furthermore, interference patterns contain more information. For example, although the separation between filaments affects the period of the fringes, it is apparent that the same is not the case with the visibility of the pattern. In Fig. III.9 one can see that in all the images, the visibility of the interference pattern is very similar, so it is apparent that this parameter is not dependent on the lateral distance between filaments, which guarantees a high coherence between them. 

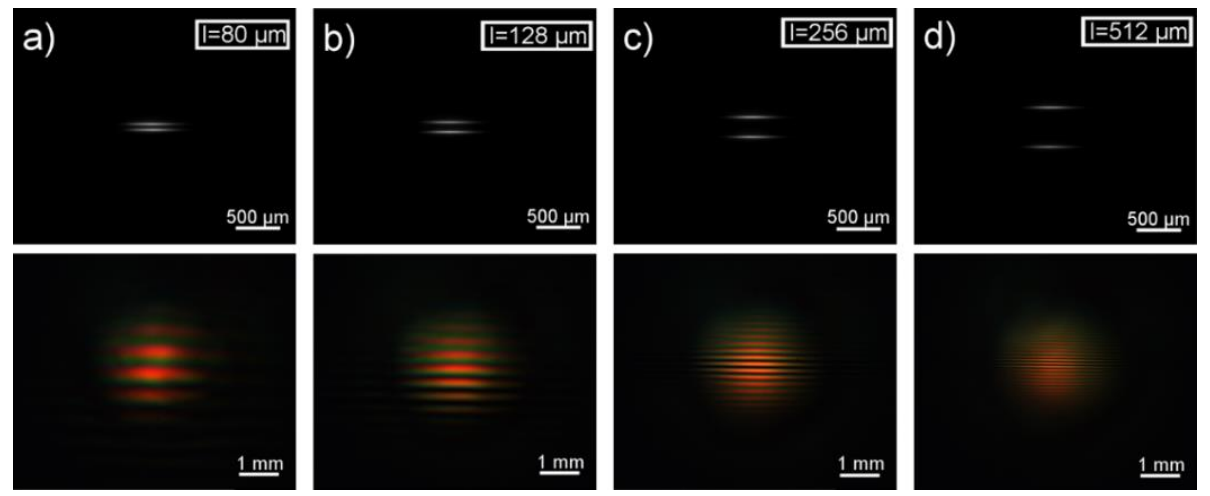

Fig. III.9 - Two filaments located at the same plane and their corresponding interference patterns with increasing lateral separations.

An arbitrary 3D distribution of filaments generates more complex interference patterns. In Fig. III.10, images of three filaments distributed as the vertices of an equilateral triangle are shown; therefore, the distance between filaments is the same. Similarly to Fig. III.9, pairs of images filaments-interference patterns are shown in the top and lower part of Fig. III.10 respectively. In addition, distances among filaments are the same as before but following the $3 D$ distribution. .From the perspective of the lateral CCD, the filaments are generated in different planes, so it seems that apparent variations of intensity in the filaments appear. However, all filaments have the same energy and this effect is produced by the different location of the filaments.
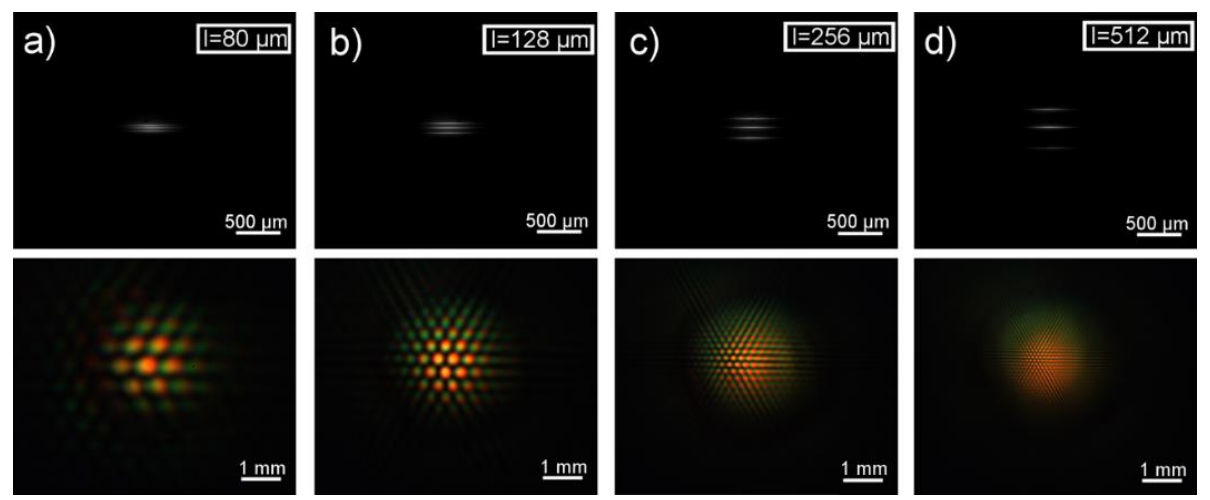

Fig. III.10 - Three filaments generated on a 3D distribution and their corresponding interference patterns with increasing lateral separations.

In this case, the interference pattern is different: instead of horizontal fringes, there is now a hexagonal-like distribution of spots. However, the correlation between the lateral separation of the filaments and the period of the 
interference pattern is similar to before. In both cases, one can see that interference patterns are color-dependent. During the experiments, it became clear that the distribution of colors in the patterns is dependent on the axial plane of generation of the filaments due two main reasons: a different penetration depth, which leads to a change on the dispersion introduced by the fused silica and different chromatic aberrations at the focal regions, obtained for different focal lengths.

It was previously mentioned that the lateral distance between filaments does not affect the visibility of the interference pattern. The next experiment is aimed to demonstrate that the axial separation of two filament, on the other hand, does affect that parameter. For this, several different images were taken using a DPE to generate two filaments in different axial positions. Specifically, five different images were recorded. In them, the axial distance $d$ between the two filaments varies from $-800 \mu \mathrm{m}$ to $+750 \mu \mathrm{m}$ thanks to the variation of the focal distance of the lens associated with the lower filament from $241.8 \mathrm{~mm}$ to $248.0 \mathrm{~mm}$. Note that the set of lenses that make up the imaging system decreases by a factor of two the transversal extension of the DPE at the output plane of the optical system. Consequently, the axial magnification of the system is $1 / 4$. For this reason, a variation in the focal distance of approximately $6 \mathrm{~mm}$ entails a variation in the axial position of the filament of approximately $1.5 \mathrm{~mm}$.
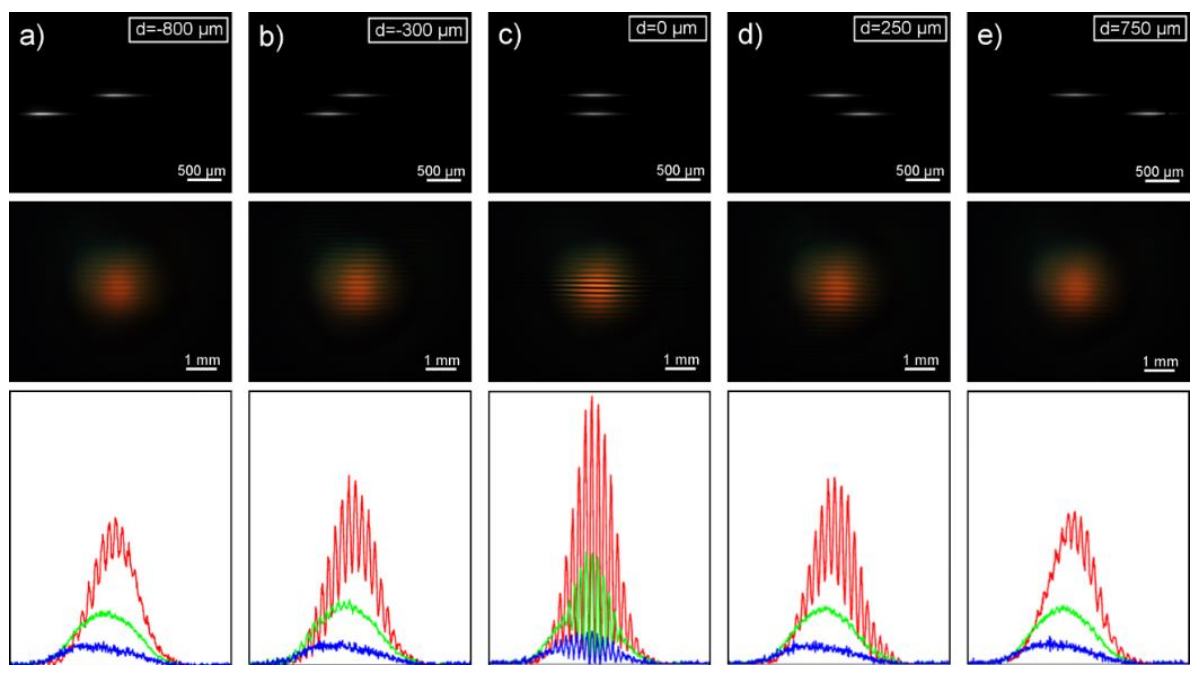

Fig. III.11 - Comparison between the interference patterns generated (middle row) by two filaments with different axial separations (top row). Additionally, $R G B$ irradiance profiles are included (bottom row). 
The results of that experiment are shown in Fig. III.11. Recorded images of the filaments are shown at the top part of the figure. The axial separation between the filaments is in accordance with the distances mentioned above. In the central row of the figure, the interference patterns generated by the corresponding filaments are shown. In order to easily compare the interference patterns, irradiance profiles taken at the central part of the interference patterns have been included.

One can notice that a different profile has been generated for each channel (red, green and blue) of the camera. This is because the interference patterns have a different behavior for each color. In addition, to improve this comparison, the values of the profiles have been normalized to the highest value, which corresponds to the red profile of figure $c$ ). This proves a higher visibility for this case, when there is no axial distance between the two filaments. Additionally, this is confirmed when the visibilities are calculated with the profiles shown in the figure, resulting in the values $0.15,0.29,0.95$, 0.35 , and 0.19 from a) to e) respectively for the red channel. Again, the maximum value corresponds to that of figure c) confirming that the visibility is reduced as the distance between filaments increases. Something similar happens with the other channels (green and blue) where, in all cases, the highest visibility is determined when both filaments are as close as possible. From a) to e), obtained results are $0.04,0.05,0.88,0.05$, and 0.06 for the green channel and $0.06,0.10,0.68,0.12$, and 0.07 for the blue one.

These results show additional information: they not only indicate the dependence of the visibility with the separation between filaments, but they also show a different visibility behavior for each RGB band. Taking Fig. III.11.C as an example, and seeing the visibility values for the red (0.95) and green (0.88) channels, it seems clear that the red channel is less affected by variations on the axial position of the filaments than the green channel, since in Fig. III.11.d the red value has dropped to 0.35 while the green one has decreased much more (0.05). When the axial position of both filaments is modified to increase the penetration depth of them within the fused silica, the visibility of each RGB channel varies in a different way. This may be produced, among other reasons, by the visible change in the azimuthal distribution of colors (conical emission) in the interference pattern caused by the variation of the penetration depth. When filaments are generated at different penetration depths, the intensity distribution of the spectral components varies. As the visibility of the interference patterns is related to the spatial and temporal 
overlapping conditions, it is affected by the modification of the penetration depth of the filaments within the fused silica.

To demonstrate this effect, Fig. III.12 shows the interference patterns generated by three distributions of filaments at three different axial positions. In all three cases, both lateral and axial separations remain unchanged, being $216 \mu \mathrm{m}$ the lateral one while there is no axial separation.
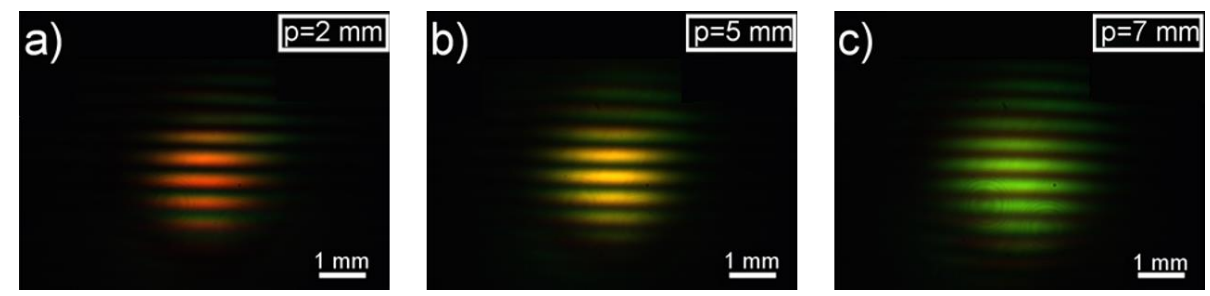

Fig. III.12 - Interference patterns generated by two filaments with no axial separation at different penetration depths within the fused silica: a) $2 \mathrm{~mm}$, b) $5 \mathrm{~mm}$, c) $7 \mathrm{~mm}$.

According to the results obtained in Fig. III.12, it is clear that the predominant wavelength in the interference pattern is dependent on the penetration depth, since the main color of the image varies as this parameter is modified. This dependency is influenced by several factors: material dispersion, initial pulse chirp, or extension of the focal length [83]. As an example, in the time domain, changes in the material dispersion entail alterations in the temporal width of the pulse. Undoubtedly, this affects the way that the filaments are generated within the fused silica, and thus modifying the associated interference patterns. Finally, it is important to remark that, to avoid a significant difference of chromatic aberrations, the focal length of the lenses used to generate the filaments is the same for all three measurements, and the moving element is the fused silica placed over a motorized stage. 


\section{Chapter IV - Complex field encoding}

In the previous chapter, a brief study on different methods to control the amplitude of a beam in a given plane was carried out. This chapter is focused on the methods that allow controlling amplitude and phase of laser beams simultaneously, especially on those techniques based on double phase method, or double phase hologram.

\section{IV.1 - General overview}

Above-mentioned techniques are limited to the control of the irradiance at the output plane. However, for some applications, it would be interesting to control not just the amplitude, but also the phase of the light wave. As reviewed on the previous chapter to reconstruct an irradiance pattern, one of the most popular solutions relies on the encoding of complex holograms. Nevertheless, complex modulation in a single phase-only SLM is not trivial. Therefore, it is necessary to use some method to modulate both the phase and the amplitude of the light wave. The first idea is to use two devices, one that modulates amplitude, while the second is used for the phase. For instance, in [92], two liquid-crystal televisions were combined with an afocal imaging system and aligned for a pixel-to-pixel matching. Tudela et al. reported a similar technique employing two ferroelectric LC SLMs to encode a complex Fresnel hologram [93]. Subsequently, other more sophisticated methods that also employ two modulators to shape arbitrary complex fields appeared. For example, in [94] two nematic SLMs are employed in a lensless configuration to shape simultaneously amplitude (using optical polarizing elements) and phase. In [95], two DOEs are encoded on conjugated Fourier planes. The first one is employed to redistribute the intensity of an incoming light beam to an arbitrary pattern by on-axis diffraction (using the GSA). This pattern is projected to a second SLM, placed at the Fourier plane of a 4 f imaging system, which is employed to perform the phase modulation of the Fourier components. In the same manuscript [95] the authors propose an alternative arrangement using just one SLM. They suggest encoding the two masks side by side in the same SLM. For this, they substitute the first lens by a slightly tilted concave mirror that reflects the light back to the SLM; the mirror sends the beam to the area of the SLM that encodes the phase. Zhu et al. reported another example of complex encoding with two SLMs in 2014 [96]. Again, one SLM is conjugated with a second one by an imaging system. By using a set of 
polarizers, they are able to control the polarization direction of the input beam throughout the entire system. In that way, the first SLM controls the amplitude of the light at the output plane of the system, while the second one only affects the phase. In all these cases, two identical devices have been used.

Single-phase SLMs are perfect for easily encoding phase patterns, while DMDs are basically aimed to manipulate the amplitude. The combination of these two elements in the same optical setup can create a fully complex modulated wavefront [97]. Several disadvantages are implicit with these strategies. First, the economic cost of two optical devices makes it advisable to use another technique that only requires a single device. Moreover, when one device should be imaged onto a second one, there is a high dependence between the alignment precision and the quality of the reconstruction. In particular, the corresponding pixels of both devices must be coupled to avoid unwanted problems.

Complex modulation with a single DMD has also been probed successfully [98]. A method called superpixel employs this kind of devices combined with a spatial filter to independently modulate amplitude and phase at a very high frame rate, since DMDs are comparatively much faster than SLMs. The main idea is to merge groups of micromirrors into superpixels and use a $4 \mathrm{f}$ imaging system made up of two slightly off axis lenses. The spatial filter, placed at the Fourier plane of this $4 f$ system, is employed to block the high spatial. In that way, the phase response of each micromirror on a superpixel is dependent on its position within the $\mathrm{DMD}$, and the response of a superpixel at the output plane can be obtained from the sum of the micromirrors. The efficiency is the main drawback of this kind of methods, since reported values are around $5 \%$.

Compared to DMDs, SLMs are slower devices; however, multiple techniques developed to encode complex fields in phase-only devices are more efficient than their counterparts for DMDs. Some of the iterative techniques discussed in the previous chapter are able to have certain control over the phase of the complex field. This is the case of the aforementioned WGS-PC, an IFTA with phase control. In a similar way, another iterative method, but this time based on a conjugate gradient minimization technique has also been reported as alternative to generate patterns with independent control over the phase and the amplitude of light [99]. Basically, this algorithm defines a cost function based on the difference between the calculated electric field and the desired pattern. Unlike IFTA based algorithms, which are based on Fourier transforms, this is a minimization algorithm that calculates the error for each pixel. A point 
in favor to this technique is that the minimization approach facilitates convergence. In general iterative algorithms, present some notable disadvantages. Specifically, obtained results are only an approximation of the target complex field. In addition, compared to other techniques more computing time is necessary for reliable results.

Another approach proposed a spatial cross-modulation method (SCMM) [100]. SCMM uses a phase-only SLM and a random phase diffuser to encode a complex object as a scattered phase image by letting the object to transmit through the random diffuser. Basically the technique is executed in two steps: digital encode step and optical decode step. The first one is developed completely by computer algorithms and its objective is to obtain a phase pattern to be encoded into the SLM. To do this, they arbitrarily define both the amplitude and phase of a complex field, which is Fourier transformed. Then the result is multiplied by the spatial phase distribution of a random phase diffuser. Through an inverse Fourier transform of the above result, a diffusion image is obtained at the output plane of the system. Finally, the amplitude of the diffusion image is ignored whereas the phase term is conjugated to obtain an image referred to as cross-modulated image. This cross-modulated image is the final element encoded into the SLM. The second step is intended to be experimental. The SLM is placed at the input plane of a $4 \mathrm{f}$ imaging system with the random diffuser placed at the Fourier plane. In that way, the desired complex field is retrieved at the output plane of the $4 \mathrm{f}$ imaging system. As one can notice, this method is quite similar to phase conjugated techniques. Some advantages of this method are the suppression of speckle noise and a high diffraction efficiency. As the encoded pattern lacks of the abrupt phase discontinuities typical of other methods, most of the light is confined to a single diffraction order and there is no need to filter out other orders. There is a compromise between the quality of the reconstruction and the pixel size of the object. The higher the ratio between the spatial resolution of the diffuser and the desired complex object, the greater the quality of the reconstruction. In order to increase that ratio, it is possible to reduce the resolution of the target object, despite this is generally not desirable.

A theoretical exact solution was proposed by Bolduc et al. [101]. However, computational cost was so intensive that, in practice, they needed an approximation for experimental realization. This finally results in an adjustment of [78], with an additional phase term, allowing the method to control both phase and amplitude. Nevertheless, this adjustment no longer provides an exact solution. More recently, a random technique was employed 
to sample two functions [102]. The first function contains the phase of the desired complex field, whereas the second one is a diverging optical element aimed to control the amplitude by redirecting undesired light out of the optical axis. Two complementary random binary patterns are generated to choose, for each pixel, which function is encoded. This technique does not require iterative algorithms or computational costly calculus making it suitable for certain applications, such as visual optics [103].

\section{IV.2 - Double phase techniques}

A large part of techniques developed to encode complex fields are based on the well-known double phase hologram (DPH) theory. First proposed by Hsueh et al. in 1978 [104], basically describes how to perform the decomposition of a complex hologram into two phase terms. This method will be explained in more detail later, but for now, we will review some techniques, reported in recent years, based on this strategy.

Among the different options, one possibility is based on the encoding of the two phase terms in two spatially separated areas of the SLM, which are superposed using a grating filter at the Fourier plane of a $4 \mathrm{f}$ imaging system [105]. This technique is based on the splitting of the SLM surface in two symmetrical regions. Something similar was proposed by Liu et al. [106]. They suggested to encode two position-shifted amplitude holograms in a single SLM, and couple them by adding a grating at the Fourier plane. It displays a complex Fresnel hologram at the output plane. Other techniques sample the phase terms to encode both phase terms simultaneously along the full surface of the modulator. For instance, one reported technique consists in the alternative encoding of the two phases in a single spatial light modulator. In that way, two adjacent pixels contain information about the two phase terms. Then, by using polarization sensitive components, these pixels are superposed for interference [107]. This solution provides a direct optical transform of pure phase values into complex values with high diffraction efficiency. A similar approach employs an additional carrier spatial frequency and a spatial filter [108]. On the same direction, but this time combining four neighboring pixels into one superpixel, it is possible to decouple the modulation of the phase and the amplitude to be able to modulate them independently [109]. These pixels are aligned in a way that their phases are shifted $\pi / 2$. Two of them are employed to construct the amplitude term whereas the remaining two modulate the phase. A spatial filter at the Fourier plane of a lens is employed to superpose the fields from the individual pixels. In general, this pixel 
combining method entails a loss of spatial resolution by half of the pixels of the SLM. Arrizón also reported a modification of the double-phase technique, also based on encoding of complex values over two neighboring pixels. It allows to perform the complex modulation with twisted-nematic LCD, but it requires at least a $2 \pi$ range of modulation [110].

As one can guess from the previous references, the encoding of DPH theory can be done in several ways: using two cascading modulators, using two SLMs properly placed in a two-arm interferometer, or by encoding both terms onto the same SLM to make them latter interfere as a CPI. This last option has several advantages over the other alternatives: first, it is more cost-effective, as long as the additional elements needed to make the interference are cheaper than a second SLM. Secondly, a CPI has some clear benefits over a two-arm arrangement, as it is more robust against mechanical vibrations, temperature changes, or optical misalignments.

Double phase methods has been successfully used for different applications including generation of speckleless holographic displays [111], optical trapping of micro-particles by generating controllable light capsules [112], demonstrate the Talbot self-imaging in the azimuthal angle [113], shaping threedimensional vector beams with arbitrary intensity distribution and controllable polarization [114], and generation of specifically designed Airy beams like elliptical Airy beams [115] or multifocus autofocusing Airy beams [116].

\section{IV.3 - Shaping the amplitude and phase of laser beams}

Our contribution [117] combines double-phase hologram theory with a spatially filtered $4 \mathrm{f}$ optical system. To fully understand how the method works, it is important to go deeper into the double phase hologram theory. It states that any complex field $\mathrm{U}(x, y)=A(\mathrm{x}, \mathrm{y}) \mathrm{e}^{i \varphi(x, y)}$ can be rewritten as:

$$
U(x, y)=B e^{i[\varphi(x, y)+\theta(x, y)]}+B e^{i[\varphi(x, y)-\theta(x, y)]}
$$

where

$$
\theta(x, y)=\cos ^{-1}\left[A(x, y) / \mathrm{A}_{\max }\right]
$$

$A_{\max }$ is the maximum of $A(x, y)$ and $B=A_{\max } / 2$. Hence, for $A_{\max }=2$ and $B_{\text {max }}=1$, the complex field $U(x, y)$ can be obtained from the coherent sum of the uniform waves $e^{i \vartheta_{1}(x, y)}$ and $e^{i \vartheta_{2}(x, y)}$, where: 


$$
\vartheta_{1}(x, y)=\varphi(x, y)+\theta(x, y)
$$

and

$$
\vartheta_{2}(x, y)=\varphi(x, y)-\theta(x, y)
$$

Note that, the desired complex field only depends on two phase terms $\vartheta_{1}(\mathrm{x}, \mathrm{y})$ and $\left.\vartheta_{2}(\mathrm{x}, \mathrm{y})\right)$, which is easier to achieve with phase-only modulators.

In our proposal, to get a single phase mask, both uniform waves $e^{i \vartheta_{1}(x, y)}$ and $e^{i \vartheta_{2}(x, y)}$ are spatially multiplexed with the help of two checkerboard patterns $M_{1}(x, y)$ and $M_{2}(x, y)$ (Fig. IV.1). These patterns can be regarded as complementary binary gratings. It means that the condition $M_{1}(x, y)+M_{2}(x, y)=1$ holds.
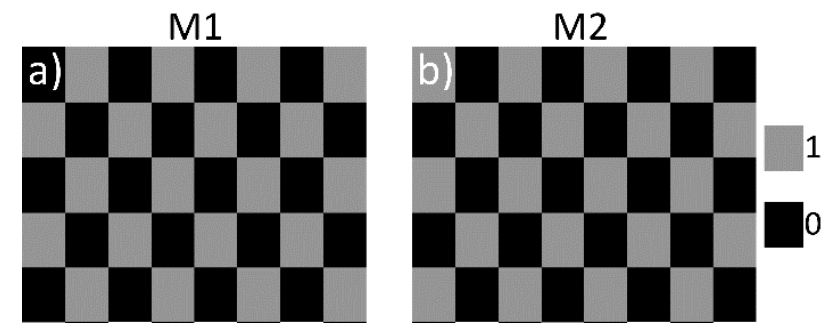

Fig. IV.1 - Example of two complementary checkerboard patterns employed to sample $\vartheta_{1}$ and $\vartheta_{2}$ phase patterns.

a) represents $M_{1}$ whereas $\left.b\right)$ represents $M_{2}$.

$$
M_{1}(x, y) \mathrm{e}^{\mathrm{i} \vartheta_{1}(x, y)}+M_{2}(x, y) \mathrm{e}^{\mathrm{i} \vartheta_{2}(x, y)}=e^{i \alpha(x, y)}
$$

In this way, a single phase element $\alpha(x, y)$ is obtained:

$$
\alpha(x, y)=M_{1}(x, y) \vartheta_{1}(x, y)+M_{2}(x, y) \vartheta_{2}(x, y)
$$

However, at this point, information of both uniform waves, contained in the phase element $\alpha(x, y)$, is not mixed but spatially separated. To force the interference to take place, a spatial filter able to block all diffraction orders but the zeroth one is employed. After the spatial filter, the spectrum of the 
encoded phase $H(u, v)=F\left\{e^{i \alpha(x, y)}\right\}$ and the spectrum of the complex field $F\{U(x, y)\}$ are linked by the expression:

$$
H(u, v) P(u, v)=\frac{F\{U(x, y)\}}{2}
$$

where $F\{\}$ represents the Fourier transform operation, $(u, v)$ refers to coordinates on the frequency domain and $P(u, v)$ holds for the spatial filter placed at the Fourier plane of the imaging system. Then, at the output plane of this imaging system, the retrieved complex field $U_{R E T}(x, y)$ is obtained by the convolution of the defined complex field $U(x, y)$ with the Fourier transform of the filter mask:

$$
U_{R E T}=U\left(\frac{-x}{m a g}, \frac{-y}{m a g}\right) \otimes \mathrm{F}\{P(u, v)\}
$$

mag is the magnification of the imaging system. Finally, $\otimes$ represents the convolution operation. This demonstrates that, apart from a slight loss of spatial resolution caused by the convolution operation, the complex field $U(x, y)$ is fully retrieved at the output plane of the imaging system.

The experimental setup designed to generate arbitrary complex fields is shown in Fig. IV.2. As previously mentioned, the base of the setup is a $4 \mathrm{f}$ imaging system. The beam of a Ti: sapphire laser is conveniently attenuated with neutral filters and magnified with a beam expander (both elements are not shown in the figure) to fit the size of the SLM screen and avoid any potential damage. A beam splitter (BS) is used to send the laser beam to the SLM with normal incidence. The SLM, which is encoding the phase element $\alpha(x, y)$, reflects back the beam towards the $4 \mathrm{f}$ imaging system. In addition, the SLM position coincides with the input plane of the system. Two lenses $L_{1}=300 \mathrm{~mm}$ and $L_{2}=150 \mathrm{~mm}$ make up the imaging system, resulting in a magnification in the output plane of $m a g=1 / 2$. 


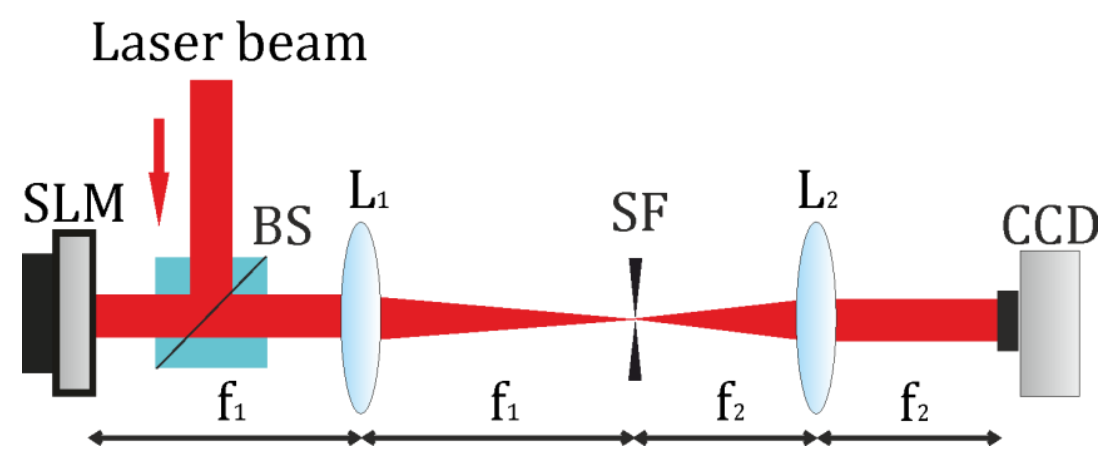

Fig. IV.2 - Experimental setup employed to generate an arbitrary complex field. The SLM, placed at the input plane of a 4 f imaging system is encoding a phase mask that generates the complex field at the output plane, where a CCD camera is placed to record the amplitude.

At the Fourier plane of $L_{1}$, a spatial filter is placed to filter all diffraction orders but the zeroth one. In our setup, a circular iris is employed for this purpose. It is advisable to adjust the aperture of the iris experimentally: once an arbitrary complex field has been defined, and the corresponding phase element $\alpha(x, y)$ has been encoded, one should vary the diameter of the aperture until the best image is obtained at the CCD camera. To measure the phase several options can be considered, including using a specific device (for instance: a Shack-Hartmann sensor). In our case, the CCD is employed to not only record the amplitude, but also the phase. In this regard, a polarizationbased phase shifting technique is employed [118]. As this technique requires two additional polarizers, both elements has been added to the experimental setup. The first one should be located before the SLM, whereas the second one is placed just before the CCD. The setup employed to simultaneously measure amplitude and phase is shown in Fig. IV.3. 


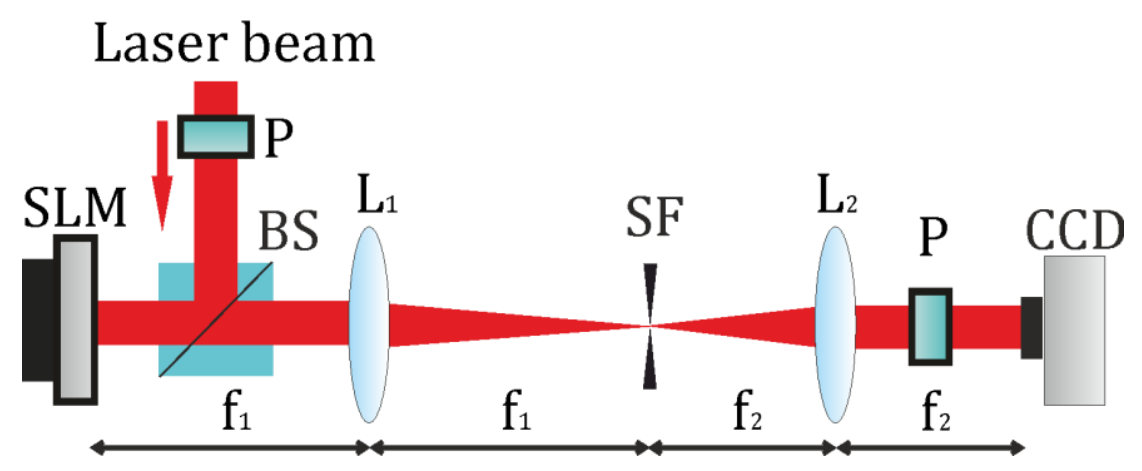

Fig. IV.3 - Experimental setup employed to measure the complex field generated by the method. In addition to the previous setup, two polarizers ( $P$ ) are included. The first one is located before the beam splitter (BS), and the second one is situated just before the CCD.

Basically, the idea around the phase shifting technique lies in recording four different interferograms with different phase shifts between the light diffracted by the SLM and the zero order (or non-diffracted light). The first polarizer allows passing a similar amount of both vertical and horizontal polarized light. In the case of our SLM, only horizontally polarized light is diffracted by it, so increasing the amount of vertically polarized light also increases non-diffracted light. As the final objective is to record the interferograms, the first polarizer is rotated to equalize both signals and thus, maximize the quality of the interferograms. The second polarizer is employed to mix both horizontally and vertically polarized signals in such a way to get interference. For this purpose, the polarizer is also rotated to equalize the two beams. To record the first interferogram, the phase mask $\alpha(x, y)$ is sent to the SLM and the CCD registers the received intensity. For obtaining the other three measurements, the phase masks $\alpha(x, y)+\pi / 2, \alpha(x, y)+\pi$, and $\alpha(x, y)+3 \pi / 2$, are sent to the SLM. Finally, by using Eq. $\mathrm{X}$ and $\mathrm{X}$, amplitude and phase of the generated complex field are retrieved.

$$
\begin{gathered}
A(x, y)=\sqrt{\left[I_{3}(x, y)-\mathrm{I}_{1}(x, y)\right]^{2}+\left[I_{4}(x, y)-\mathrm{I}_{2}(x, y)\right]^{2}} \\
\varphi(x, y)=\arctan \left[\frac{I_{4}(x, y)-\mathrm{I}_{2}(x, y)}{I_{3}(x, y)-\mathrm{I}_{1}(x, y)}\right]
\end{gathered}
$$

where $I_{1}, I_{2}, I_{3}$, and $I_{4}$ are the four interferograms recorded following the previously mentioned procedure. 
In Fig. IV.4, an example of an experimental result is shown. On the top part of the image (Fig. IV.4.a and 4.4.b), defined amplitude and phase of the complex field are respectively shown. The amplitude image is the well-known Lenna image (which was not so controversial at the time this experiment was performed), whereas the phase image is the picture of a little girl. In Fig. IV.4.C, appear the four interferograms $I_{1}, I_{2}, I_{3}$, and $I_{4}$, employed to retrieve the complex field using Eq. (III.9) and Eq. (III.10). Fig. IV.4.d and 4.4.e are the obtained amplitude and phase at the output plane of the system.

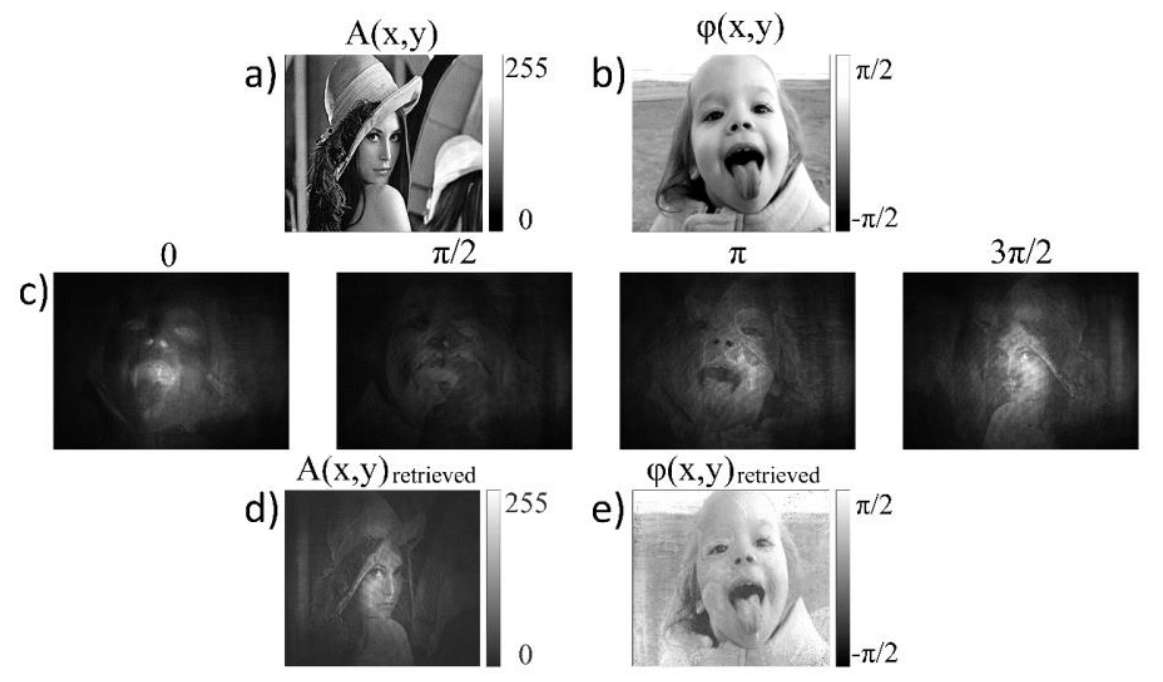

Fig. IV.4 - Experimental results using quasi-monochromatic illumination. a) Desired amplitude of the complex field. b) Desired phase of the complex field.

c) Interferograms $I_{1}, I_{2}, I_{3}$, and $I_{4}$ recorded with the phase shifting technique [118]. d) retrieved amplitude of the experimental complex field. e) retrieved phase of the experimental complex field.

For this experiment, to emit a quasi-monochromatic laser radiation, the Ti:Sapphire laser is employed out of the mode locked condition. In particular, the measured full width half maximum of the intensity is about $10 \mathrm{~nm}$ (centered at $800 \mathrm{~nm}$ ).

The quality of the obtained images is determined by the similarity between the desired and the retrieved images. The difference between the defined phase and the experimental one in the central region of the image is about $7 \%$ on average. The normalized amplitude is also compared, resulting in a measured error close to $8 \%$. The main reasons behind these discrepancies are the non- 
uniform intensity of the incident laser beam and the distortion given by the loss of spatial resolution due to the filtering process. One can expect a more accurate reconstruction of the complex field if the complete surface of the SLM is filled with uniform light.

Spatial resolution is affected by the pixel pitch of the SLM. Obviously, the smaller the pixel size of the SLM, the better the spatial resolution of the obtained amplitude and phase patterns. However, reducing the pixel size is not trivial because reducing the spatial separation among them can increase the effect of an electric field addressed to a pixel over the contiguous pixels. This inconvenience will be less and less important when SLMs with smaller pixel sizes appear. In another vein, a known and recurring problem in this type of methods is related to pixel cross-talk. Basically, it should be remembered that abrupt phase changes between contiguous pixels of the SLM can generate unexpected phase responses. The use of checkerboard patterns to intercalate the phase terms $\vartheta_{1}$ and $\vartheta_{2}$ generates the abrupt variations that favor this effect. To minimize this issue, pixels of each checkerboard pattern are grouped in superpixels or pixel cells (Fig. IV.5).

According to Eq. (III.6), phase mask $\alpha(\mathrm{x}, \mathrm{y})=\vartheta_{1}(x, y)$ if $M_{1}(\mathrm{x}, \mathrm{y})=1$, and $\alpha(\mathrm{x}, \mathrm{y})=\vartheta_{2}(x, y)$ if $M_{1}(\mathrm{x}, \mathrm{y})=0$ (please, note that checkerboards patterns $M_{1}$ and $M_{2}$ are complementary).
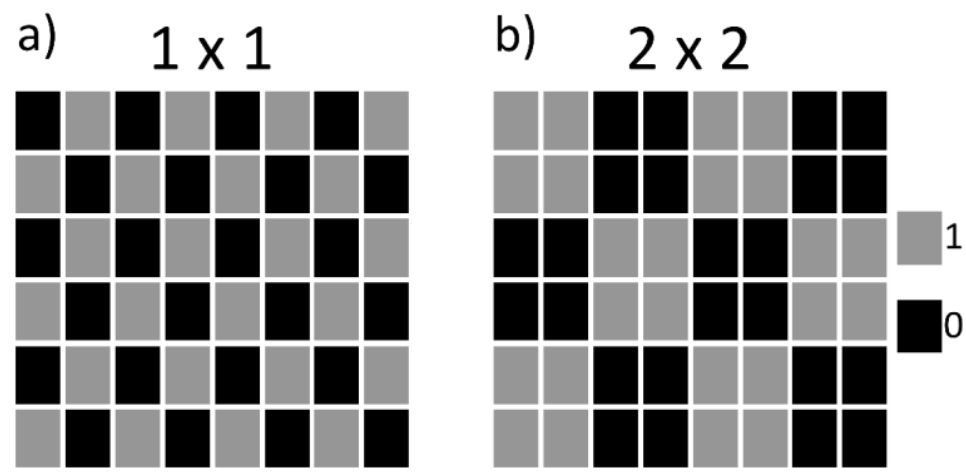

Fig. IV.5 - Example of grouping pixels into superpixels. Two $M_{1}$ checkerboard patterns are shown: a) pixel cell $=1 \times 1$. b) pixel cell $=2 \times 2$.

Checkerboard patterns shown in Fig. IV.5.a and 4.5.b represent different options for the mask $M_{1}$. In the first case (Fig. IV.5.a), no superpixel grouping is employed, so two contiguous pixels would always have different values. This 
is, the first pixel (the one at the top left corner) has a value $0: M_{1}(0,0)=0$, and then, because both masks are complementary: $M_{2}(0,0)=1$. As the pixel cell is $1 \times 1$, all their adjacent pixels will take the value 1 . For instance, the pixel $M_{1}(0,1)=1$. According to Eq. (III.6), then $\alpha(0,0)=\vartheta_{2}(0,0)$ while $\alpha(0,1)=\vartheta_{1}(0,1)$. The phase variation between both pixels be abrupt resulting in pixel cross-talk effects. The phase variation would be almost certainly less if $\alpha(0,0)=\vartheta_{1}(0,0)$ and $\alpha(0,1)=\vartheta_{1}(0,1)$, as phase variations among contiguous pixels of the same phase mask tend to be smoother.

Instead of using 1x1 checkerboard patterns, what we proposed is to group those pixels into superpixels. In that way, a group of nearby pixels belongs to the same pixel cell. For instance, in Fig. IV.5.b, the pixels of the SLM has been clustered into $2 \times 2$ superpixels. The first pixel (the one at the top left corner) has one pixel on its right and another one below that pertains to the same superpixel. In this example, $\mathrm{M}_{1}(0,0)=1$ and $\mathrm{M}_{1}(0,1)=1$, as both belong to the same superpixel. Therefore, in Eq. (III.6) $\alpha(0,0)=\vartheta_{1}(0,0)$ and $\alpha(0,1)=\vartheta_{1}(0,1)$ which commonly results in a smaller phase change.

On the one hand, we have found that above process effectively reduces the harmful effect of pixel cross-talk. On the other hand, from Eq. (III.8) increasing the size of the superpixel decreases the spatial resolution of the retrieved amplitude and phase patterns. Therefore, the choice of superpixel size implies a compromise between alleviating cross-talk effects and reducing the spatial resolution of the complex field generated. An SLM with a lower pixel width would allow reducing the pixel cross-talk effect, without a significant loss of spatial resolution.

Another important parameter related with the SLM is the phase range. The available range could limit the capability of the SLM to encode the desired amplitude $A(x, y)$ or phase $\varphi(x, y)$. In our case, the phase of the complex field is defined from $-\pi / 2$ to $\pi / 2$, which implies an absolute phase range of $\pi$. The same happens with the amplitude, defined by an arccosine function, and therefore in the range $(0, \pi / 2)$. The final mask is calculated by adding and subtracting the arccosine of the amplitude to the phase terms (Eq. (III.3) and Eq. (III.4)) resulting in a final total range of $2 \pi$. In short, that means that a minimum phase shift of $2 \pi$ is needed to obtain the results presented here. However, if only a device with lower phase range is available, it is possible to apply some constraints during the encoding process. In the opposite case, if 
an SLM with a higher range is available, one can encode the phase with greater precision.

A second experiment was performed, but this time, instead of using quasimonochromatic illumination, ultrashort pulsed illumination was employed. In a very similar way to the previous results, only one single phase mask $\alpha(x, y)$ is employed to modulate the different spectral components of the broadband light source. Although the laser radiation remains centered at 800 $\mathrm{nm}$, this time the spectral bandwidth is about $100 \mathrm{~nm}$ full width half maximum.

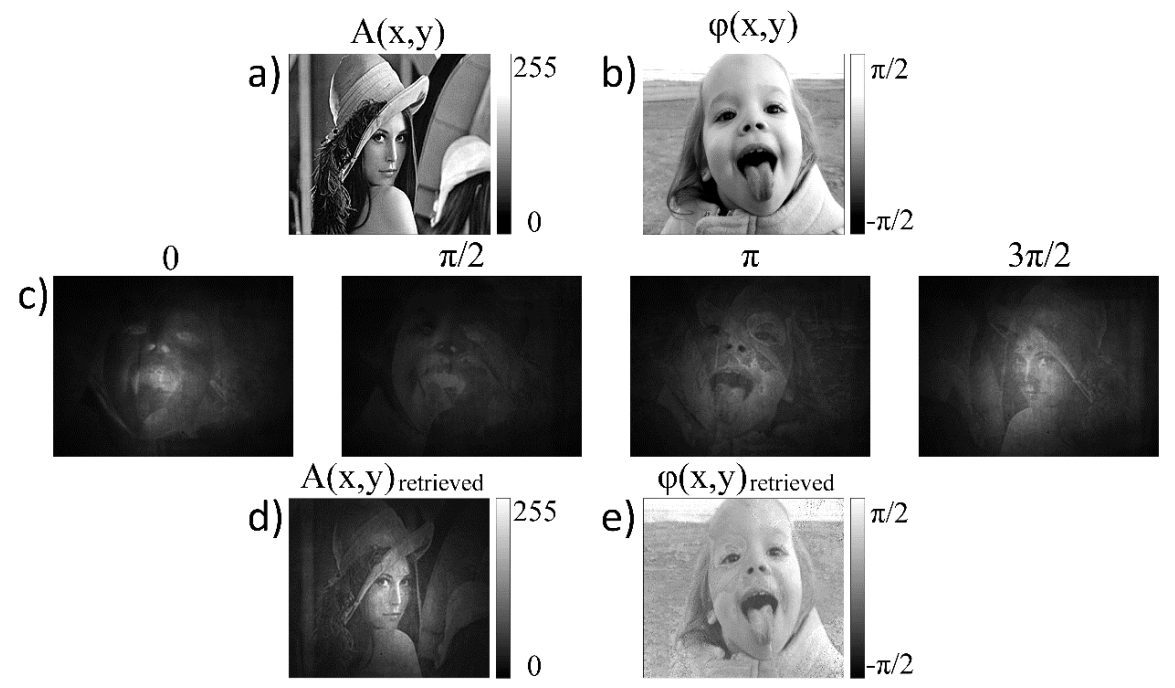

Fig. IV.6 - Experimental results using ultrashort pulses. a) Desired amplitude of the complex field. b) Desired phase of the complex field. c) Interferograms

$I_{1}, I_{2}, I_{3}$, and $I_{4}$ recorded with the phase shifting technique [118]. d) retrieved amplitude of the experimental complex field. e) retrieved phase of the experimental complex field.

At a glance, one can notice that the results are quite similar besides of the different illumination employed. Obviously, minor differences can be observed on both amplitude and phase images with respect to the previous experiment. The metrics show a slight reduction of quality compared to the previous measurements. The measured gray levels differ about a $9 \%$ of the theoretical values (versus a 7\% in Fig. IV.4). The difference among the expected and the reconstructed phase of the complex field is also moderately higher in this case. The comparison results in a bigger difference using ultrashort pulses (about $10 \%)$ than quasi-monochromatic illumination (8\%). However, the small 
difference reported by these results suggests that the proposed technique is also able to manipulate ultrashort pulses.

A very interesting topic related with this kind of techniques is the management of the zero order. As it was previously mentioned, the zero order can introduce some unwanted distortions to the recorded complex field since it contains non-diffracted light. In that way, it is not possible to control with the SLM neither the amplitude nor the phase of this type of light. Commonly, when using on-axis optical systems, non-diffracted light remains spatially distributed along the whole image. In any case, there are options to try to minimize this harmful effect. Some approaches take advantage of the inalterability of that light to remove it from the system. For instance, it is possible to separate the zero order noise from the zero order information by designing an off-axis optical system [111]. In this case, a blazing grating is added to the mask $\alpha(x, y)$ for this purpose and, once the noise and the information are separated, the noisy part is blocked by a spatial filter at the Fourier plane of the $4 \mathrm{f}$ imaging system commonly employed on this type of systems. Although it is true that the non-diffracted light is significantly reduced and the diffraction efficiency should be, theoretically, the same, in practice there is a reduction of this parameter. The problem lies on the pixelated structure of the SLM. An ideal grating can maintain a diffraction efficiency of almost $100 \%$. However, the ideal grating is a continuous function that ranges from 0 to $2 \pi$ with perfect precision. The SLM can only approach this function since it cannot encode this continuous profile over such small intervals due to the pixel size of the SLM. The smaller the pixel size, the better the approximation made since the diffraction efficiency of the grating depends largely on the number of levels that used to approach a phase shift of $2 \pi$. On the -1st order, a two level grating produces diffraction efficiencies about 40\%; four levels produce diffraction efficiencies about 81\%, whereas eight levels can produce diffraction efficiencies about 95\% [119].
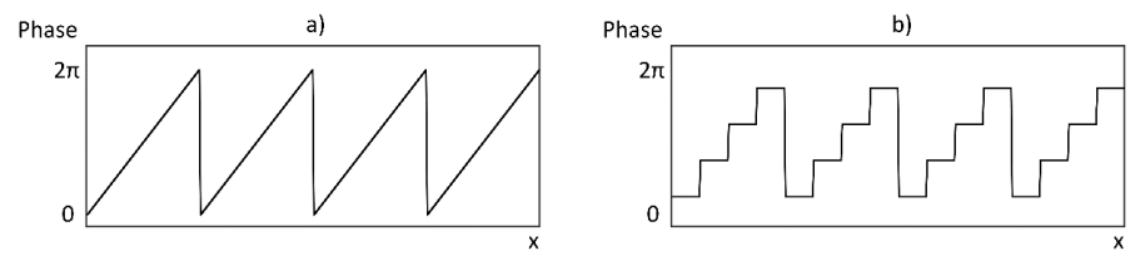

Fig. IV.7-Ideal grating (a) vs four levels discretized grating (b).

In Fig. IV.7 a comparison between the phase profile of an ideal grating (continuous) and the phase profile of a multi-level phase structure, in this 
case a four level profile, is shown. In this figure, it is visible why the number of levels is so relevant in the diffraction efficiency of the grating: the more levels, the more accurate is the approximation to the ideal grating. So, to sum up: this technique is advisable if at least eight phase levels are available to encode each $2 \pi$ range, otherwise one should assume that there would be a relevant intensity reduction. 



\section{Chapter V - Applications of complex field encoding}

This chapter describes some of the applications in which modulators are an important part to achieve the proposed objectives. Specifically, those applications resulting from complete control of the complex field are especially relevant for the purpose of this thesis. First, many of the applications of the spatial complex beam shaping are revised. After that, we focus on microscopy since several efforts done during this thesis have been focused on that field. Finally, two published manuscripts resulting from that work are presented.

The first one generates an arbitrary distribution of three-dimensional foci. In this case, Fresnel lenses are coherently added resulting in a complex field. The distribution of foci was used to generate non-linear effects in arbitrary positions within a three-dimensional volume. That could be used, for instance, in the illumination path of a microscope. In the second manuscript, the double phase method is employed to generate complex illumination patterns to trigger non-linear effects in extended ROls. Additionally, control over the phase is used for various purposes, including correction of aberrations and interference analysis.

\section{V.1 - Main applications}

Over the last decades, SLMs have been used extensively in many fields. These devices have been included to improve many topics and have allowed the development of new techniques that broaden the number of achievable applications. Two examples are aberration correction and optical pulse shaping. Commonly, optical setups introduce a certain distortion in the phase of the beam. Small misalignments or imperfections on the surfaces of the different optical elements are usually behind these types of aberrations, reducing the performance of the designed setup. An SLM can be used to precompensate some optical aberrations. For instance, if the phase-only SLM is imaged onto a certain plane, it can correct the aberrations at this plane by encoding the conjugated phase of the measured aberration (see. Fig. V.1). 

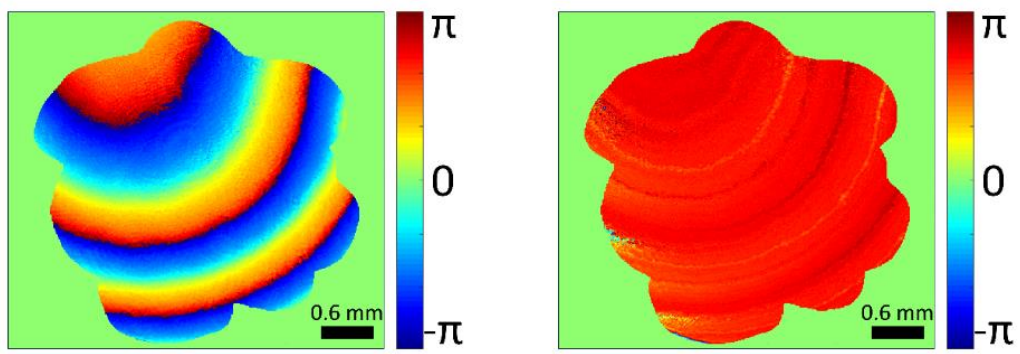

Fig. V.1-Recorded phase of a laser beam without (left) and with aberrations correction (right).

Moreover, the same SLM can be used to solve this problem while performing another task simultaneously (encoding a lens, generating a $\mathrm{CGH}$, etc.). This is especially useful for certain purposes including focusing systems, where the aberrations can cause the beam to not focus to a sharp spot, or hologram projection, where sometimes it is difficult to reconstructing sharply focused images. In addition, when the beam is expected to pass through an object, the SLM can be employed to correct the optical aberration introduced in the object. In this way, one can correct the aberrations in the human eye [120] or improve laser machining inside of an object [121].

Pulse shaping is also benefited from SLMs. The pulses emitted by a femtosecond light source are extremely short events. In fact, there is no electronic device able to directly manage them because there is no device able to work with response times on the order of the femtoseconds. This leads to the development of techniques that allow the control of these pulses in the time domain. Thanks to SLMs, it is possible to achieve programmable compression of chirped optical pulses, arbitrary pulse retardation and other desirable effects. To do that, the pulses can be transformed to the frequency domain by means of Fourier transforms, then it is possible to filter the desired frequencies, and finally brought back to the temporal domain with an inverse Fourier transform [122]. Although this can be done without phase control, independent control of both amplitude and phase on the frequency domain can enhance the control over the waveform.

In addition to the applications just mentioned, arbitrary manipulation of the complex field of a wavefront has opened up new avenues for several applications. Probably one of the most intuitive is holographic display [123]. An arbitrary scene is defined and then, using diffraction theory, the complex field at the hologram plane is calculated. This complex field can be encoded into the 
SLM to reconstruct the three-dimensional scene by using the techniques mentioned on the previous chapter. Other relevant application is the generation of holographic tweezers $[124,125]$. In this case, the precise handling ensures fine control over scattering and gradient forces with very high efficiencies. In optical information processing, the SLM is employed to modulate a two-dimensional optical signal that becomes an information carrier [126]. Arbitrary spatially variant polarization modulation of a light wave can be obtained by dividing the surface of the SLM on two, and by doublepassing a beam through these two areas [127]. Moreover, material processing is another interesting application. If there is enough energy available it is possible to process a material once the shape of the laser beam can be controlled in an optical setup. This can be done for instance with a focusing system and a scanning device. Unlike scanning methods that are more timeconsuming, an SLM can speed up the procedure since it can process a wide region simultaneously. In addition, the use of the SLM can be combined with those scanning methods to increase the processing area while still reducing the temporal requirements of the algorithm [128]. Here, the SLM is employed to dynamically generate many lower energy beams from a single laser beam. Multiple spots can be independently manipulated allowing to carry out precise micro-structuring processes with increased throughput and efficiency. For instance in Fig. V.2, an example of laser material processing is shown. In this case, the ablation process of each pattern is performed simultaneously in the whole surface in a single-shot regime.

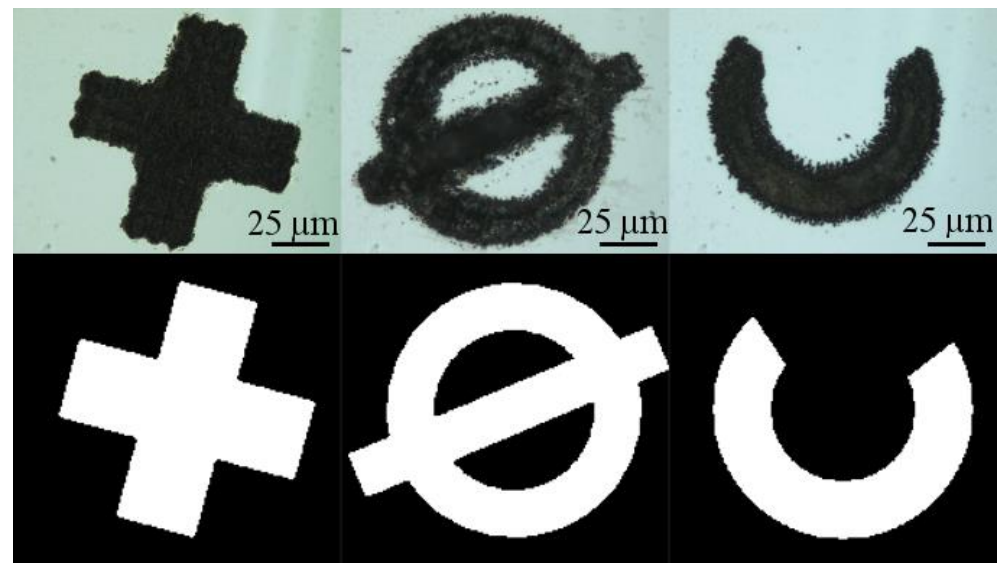

Fig. V.2 - Example of three laser-processed patterns. Top row shows the experimental results whereas the low row shows the desired pattern. Image adapted from [129]. 


\section{V.2 - Microscopy}

Finally, it is truly relevant the contribution of this type of techniques to microscopy due to its versatility [11]. In microscopy, SLMs can serve several purposes: they can be employed to control the illumination over the sample or in the imaging path, even both simultaneously. As this thesis is aimed to the generation of arbitrary complex fields, here we focus deeply on the use of SLMs for this purpose.

But prior to investigate different ways to take advantage of the complex field encoding on microscopy, it is worth commenting on some other uses where SLMs are employed to enhance the images recorded by a microscope or even to develop some new techniques. For instance, the ability of phase-only SLMs of controlling the phase at will enables the use of techniques that increases the image contrast. The main idea lies on the use of SLMs as Fourier filters. By placing a modulator properly in a certain configuration (i.e. at the Fourier plane of a $4 \mathrm{f}$ imaging system), it is possible to control the spatial frequencies, enabling the use of different contrast enhancing techniques. It is not difficult to find reports where a modulator is employed to improve the contrast of the images. Existing techniques can be adapted to be used with SLMs. This is the case of differential interference contrast [130]. Previously, this method use to employ Wollaston prisms to record two slightly displaced coherent images. These images are overlapped in a certain plane generating an interference pattern. The SLM substitutes these prisms to generate and overlap the two images of a sample. The ability of the SLM to vary dynamically the encoded masks allows changing the orientation, displacement, phase shift between images, and other parameters, enabling a fast acquisition of the interference patterns. In a similar way, under some conditions, placing the SLM in the Fourier plane of a focusing system allows to directly control the Fourier components of the beam. Blocking or shifting the zeroth-order Fourier component gives rise to different methods known as dark field microscopy or phase contrast microscopy, enabling several ways to obtain enhanced images [131].

Focused on situations in which SLMs are employed into the illumination path, but for tasks other than performing an irradiance shaping, we can find techniques like optical diffraction tomography (ODT) [132]. ODT is a technique based on illuminate a certain sample under several angles of incidence and detect the intensity of the diffracted far field from different points of observation. From this set of bi-dimensional images, it is possible to 
reconstruct the three-dimensional distribution of the sample after a proper numerical procedure. To vary the inclination angle of the illumination over the sample, one can choose among different options including DMDs [133], galvanometric mirrors [134] or LC-SLMs [135].

Another improvement is related to aberrations correction. Several effects produced by the optical system can deform the point spread function (PSF) of the objective. Slight misalignments, dirt on the surface of the optical elements, or other unwanted situations can produce defocus or displacements on the focal spot, preventing from obtaining an ideal PSF for the illumination of a microscope. SLMs can correct it by encoding a phase mask designed to correct those aberrations $[136,137]$. Thus, they allow to generate arbitrary illumination patterns and even to change them dynamically to perform sequential measurements. Simultaneously, the beam shaping can be complemented with the correction of the aberrations to improve the irradiance pattern generated over the sample. Nevertheless, this ability to change the PSF not only can be employed to obtain an ideal focus, but also to gain advantage of specifically designed ones. For instance, a double helix PSF was demonstrated as a useful tool to obtain three-dimensional singlemolecule fluorescence images beyond the optical diffraction limit [138].

Finally, due to the capability of modulators to control both amplitude and phase, SLMs are an excellent tool to control the irradiance (and phase) profile of the illumination over the sample. In this way, SLMs can be employed to encode DOEs able to perform some tasks. For instance, the precise control that SLMs provide about some physical parameters (e.g. amplitude and phase) allows controlling light propagation inside scattering media. This kind of materials generates a random scattering effect to the light that passes through them. This is mainly produced by non-uniformities present inside the whole volume of the medium. Experimentally, this effect hinders taking images of objects when a turbid media is involved. Recently, SLMs have been employed to pre-compensate the mentioned optical distortions improving the results obtained under these conditions. One of the first moves in that direction was done when focalization of coherent light through a deep layer of turbid media was demonstrated [139]. The main idea was to employ an SLM and a photodetector in closed-loop configuration to find the phase mask that optimizes the desired output. Later, a crucial step was taken when it was demonstrated the possibility of measuring the transmission matrix of any turbid medium [140], enabling not only the spatial correction, but also the temporal distortions induced by scattering [141], and the polarization state of the light 
after the medium [142]. The ability to generate a focal spot through these media is extremely relevant to achieve certain targets. For instance, if one is able to generate and displace a focus over a certain sample, it is possible to scan it. In that way, non-linear microscopy can employ these kind of techniques to recover images of a sample placed inside a turbid media [143].

But DOEs cannot only shape a single spot, but a set of them, even in different planes [144]. For instance, in this case the combination of Dammann lenses with gratings are employed to generate several 2D distributions in different planes, resulting in a 3D distribution of focus. A similar result can be obtained using a tuned version of the above-mentioned GSA. In [145], a hologram is designed to obtain multiple irradiance distributions located at different axial positions. These foci arrangements may be suitable to generate fluorescent effects to probe a 3D medium.

However, scanning techniques are known for their limited temporal resolution. In a single spot configuration, for each position of the focus, only one "pixel" of the retrieved image is obtained. Thus, to obtain the full image, a large amount of measurements should be performed. In fact, the higher the resolution, the higher the number of measurements needed to obtain it. The techniques reviewed in the previous chapters are perfect candidate to generate targeted illumination over a sample, speeding up the process. In last years, several techniques have been reported demonstrating, for instance, their usefulness to obtain fluorescence images of neurons, $[26,146]$.

A different approach to obtain three-dimensional microscopic images is based on stereoscopy. The SLM is employed to generate a fringe pattern over the sample, and a detector is used to measure the deformed pattern caused by the surface of the sample $[147,148]$. Then, usually a computer is employed to calculate the image of the sample. However, unlike other techniques, this one relies on the surface of the sample to obtain the image. On the positive side, this allows to retrieve not only the image but also the topography of the sample. By contrast, this technique cannot deep into the sample. Moreover, as just a mere fringe pattern is needed on the illumination side, other amplitude-only systems as DMDs are being employed to fulfill the same function [149].

There are more applications in which SLMs are involved in one way or another to achieve a proposed objective or to improve the results but a complete list them all is beyond the scope of this thesis. 
Finally, it is important to remark a couple of contributions published during the completion of this thesis. The first one proposes an arbitrary generation of multiple foci using the complex field encoding technique discussed in the previous chapter [117]. The second one employs the same technique to demonstrate, among other things, the possible application of the technique to nonlinear microscopy applications.

\section{V.3 - Generation of 3D foci distributions with complex Fresnel holograms}

Our first proposal uses Fresnel holograms to generate multiple arbitrary foci with micrometrical precision [150]. The main idea beyond this work is to generate a hologram from the coherent sum of multiple Fresnel lenses and encode the amplitude and phase of the complex field obtained from this sum. Thanks to the encoding technique, that ensures an optimal codification of both amplitude and phase of the complex field, the hologram is able to generate an irradiance pattern with full control over its transversal but also the axial positions. Other techniques can be employed for the same purpose: for instance, an array of lenses can be encoded into the SLM to obtain an array of foci. In addition, other techniques able to generate an arbitrary distribution of amplitude can also be employed to generate the foci distribution, as the foci is essentially an amplitude spot. However, as far as I know, none of these techniques truly encodes the complete complex field necessary to generate multifocal irradiance patterns, but obtain acceptable approximations of it. Those techniques that employ lens arrays or that multiplex them spatially generally are based on encoding only the phase information of the lenses. In the case where iterative algorithms are used, they only obtain an approximate pattern of the ideal solution. Compared to the techniques mentioned above, one able to reconstruct both amplitude and phase with pixel-level precision could increase the accuracy of the position and the quality of the generated foci.

Specifically, the reported technique proposes a three-step process to obtain the hologram that generates the desired three-dimensional distribution of foci. The first step is aimed to obtain a complex field $U(x, y)$ from the coherent sum of $N$ weighted lenses:

$$
\sum_{n=1}^{N} K_{n}(x, y) e^{i \gamma_{n}(x, y)}
$$


In that way, each lens function is described as $K_{n}(x, y) e^{i \gamma_{n}(x, y)}$ where $K_{n}(x, y)$ represents the amplitude of the lens and $\gamma_{n}(x, y)$ is the phase that can be defined by this expression:

$$
\gamma_{n}(x, y) \equiv \bmod \left\{\pi\left[\left(x-\xi_{n}\right)^{2}+\left(y-\eta_{n}\right)^{2}\right] /\left(\lambda f_{n}\right), 2 \pi\right\}
$$

where mod holds for the modulo function, $\xi_{n}$ and $\eta_{n}$ are the spatial coordinates of the center of the lens, $f_{n}$ represents the focal length of the lens, and $x$ and $y$ are the transversal coordinates. At this point, it is trivial to encode just the phase term onto the phase-only SLM. However, this would result in a loss of information leading to an inaccurate foci generation. The optimal reconstruction is obtained when the full complex field is encoded into the SLM. For that reason, in the second step the previously described technique [117] is employed. As one can know at this point, this technique is suitable to encode the full complex field. The second and third steps are related to the technique: while the second step rewrites the complex field to obtain the two phase terms (as expressed in Eq. (IV.1)), the third one uses the above-mentioned binary masks $M_{1}$ and $\boldsymbol{M}_{2}$ to spatially sample the two phase terms $\varphi(x, y)+\theta$ and $\varphi(x, y)-\theta$ (see Eq. (IV.2)).

The experimental setup designed to test this technique is conditioned by the encoding technique. Note that, to mix the two sampled phase terms from Eq. (IV.2), a $4 \mathrm{f}$ imaging system is needed. At the Fourier plane of the imaging system, a spatial filter should be placed to coherently sum the two phase terms, forming a CPI. Finally, at the output plane of the imaging system, the complex Fresnel hologram is retrieved.

As two different experiments were planned, two different experimental setups were needed. The first experiment was aimed to test the capabilities of the technique. For that reason, a phase element $\alpha$ containing a set of threedimensional distributed foci was generated. The foci were positioned in such a way that they form the characters of the word UJI (the acronym of the university). All foci were distributed among three different planes by setting different focal lengths on the defined lenses ( $f_{1}=400 \mathrm{~mm}, f_{2}=600 \mathrm{~mm}$, and $\left.f_{3}=800 \mathrm{~mm}\right)$. Thus, all the foci corresponding to the character $U$ were generated using the focal length defined by $f_{1}$, whereas the corresponding to the characters $J$ and $/$ were generated by using the focal lengths defined by $f_{2}$ and $f_{3}$, respectively. The phase element $\alpha$ was obtained following the three- 
step process described previously, resulting in the desired pattern that generates the foci distribution. The phase element is shown in Fig. V.3.

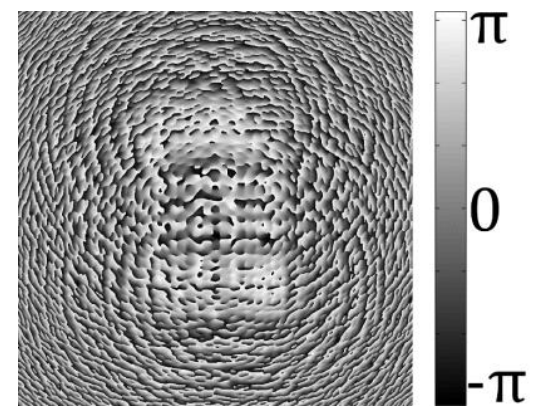

Fig. V.3-Phase element $\alpha$ encoded into the SLM to generate the threedimensional distribution of foci.

The setup is shown in Fig. V.4.

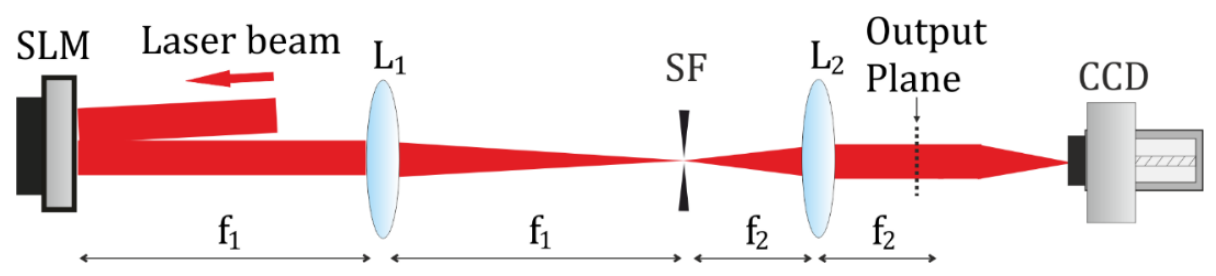

Fig. V.4 - Optical setup used to generate a three-dimensional distribution of points from a single phase element employing a $800 \mathrm{~nm}$ wavelength centered laser source.

SF: Spatial filter. L1 and L2: lenses. M1 and M2: mirrors.

In all experiments the beam of a Ti:Sapphire femtosecond laser centered at $800 \mathrm{~nm}$ is used as the illumination source. The original bandwidth is about \pm 40 $\mathrm{nm}$; however, the beam passes through a bandpass filter to reduce it to \pm 10 $\mathrm{nm}$ before impinging onto the SLM surface. By reducing the bandwidth to quasi-monochromatic, possible unwanted effects generated by the diffraction and propagation of the different wavelengths of the illumination source are avoided. Additionally, a set of neutral filters are also included within the setup to control the energy. As it was described above, a $4 \mathrm{f}$ imaging system is mandatory to obtain the complex field. For that reason, the SLM is placed at the input plane of a $4 \mathrm{f}$ imaging system formed by lenses $L_{1}$ and $L_{2}$ with focal lengths $L_{1}: \mathrm{f}=1000 \mathrm{~mm}$ and $L_{2}: f=500 \mathrm{~mm}$. The reason for employing these long focal length lenses is to reduce the angle of incidence of the beam with 
respect to the SLM display (less than 4 deg). At the output plane, the complex Fresnel hologram is obtained. Taking in account the focal lengths of the lenses (400 mm, $600 \mathrm{~mm}$, and $800 \mathrm{~mm}$ ), and the axial demagnification produced by the $4 \mathrm{f}$ imaging system (1/4), these foci can be found $100 \mathrm{~mm}, 150 \mathrm{~mm}$, and $200 \mathrm{~mm}$ after the complex Fresnel hologram. To be able to record images at the three planes, a CCD camera is mounted over a motorized stage. The stage allows displacing the camera over the three positions along the axial axis. Finally, at the Fourier plane of the imaging system, the spatial filter (a hard iris) is shown. Note that this element is essential to carry out the coherent mix between the both waves sampled onto the SLM, as a CPI.

The images recorded at the three planes are shown in Fig. V.5.
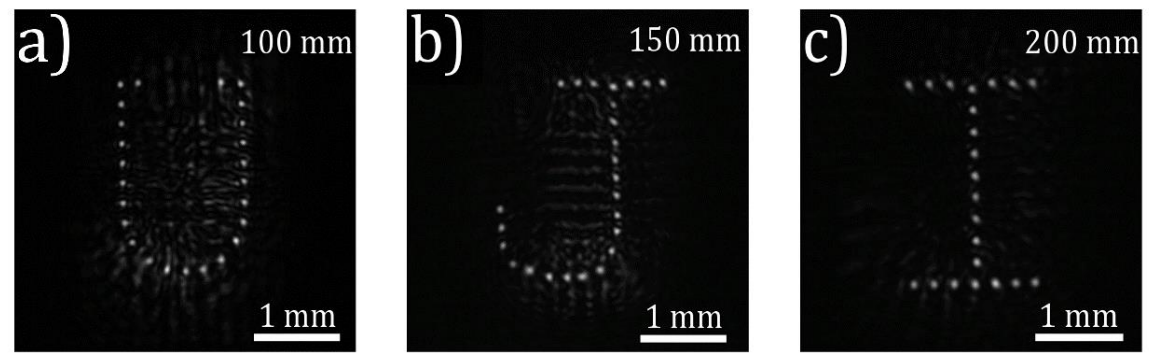

Fig. V.5 - Arbitrary three-dimensional distribution of foci obtained with a single phase element. Each image is obtained at a different axial plane. a) shows the image recorded $100 \mathrm{~mm}$ after the reconstruction plane. b) and c) show the recorded images after $150 \mathrm{~mm}$ and $200 \mathrm{~mm}$, respectively.

Fig. V.5.a shows the character $U$, recorded at the first plane $(d=100 \mathrm{~mm})$ whereas in Fig. V.5.b and Fig. V.5.c are represented the characters $J$ and $I$, on images recorded at distances $d=150 \mathrm{~mm}$ and $d=200$, respectively. At a first glance, the results reproduce properly the desired distribution in all three images. To measure the quality of our result, the following metrics will be used: Precision in the position of the foci, uniformity in the intensity of the foci, and size of the foci. The expected position and the measured position match correctly. To compare these values, the expected position is estimated by the corresponding position within the mask $\alpha$ encoded into the SLM. According with Eq. (IV.2), $\xi_{n}$ and $\eta_{n}$ represent the coordinates of the center of the lens. These data are modified according to the demagnification of the $4 \mathrm{f}$ imaging system. Finally, these values are compared with the recorded images confirming that both are very similar. In all foci, the error between the 
expected and the measured position is less than three pixels. The uniformity of the intensity of the foci can be easily measured by comparing the images recorded by the camera. The higher the measured gray level, the brighter the focus. The maximum intensity difference measured between two foci in these images is $20 \%$, which is in accordance with our target. The final metric is the size comparison between the spots. To measure the size of the foci, the $1 / e^{2}$ criterion is employed. The results obtained indicate that the measured size is different for each one of the images. In Fig. V.5.a, the foci that make up the $U$ character have an average radius of $35 \mu \mathrm{m}$. In Fig. V.5.b and 5.5.c, the average radius of the spots are $48 \mu \mathrm{m}$ and $59 \mu \mathrm{m}$, respectively. However, one must take into account that each image has been taken in a different plane. Thus, according to the equation $\Delta \omega=\lambda M^{2} \Delta f /\left(\pi \omega_{0}\right)$, the focus size depends, among other factors, on the focal length of the lens. In particular: the increment on the radius $(\Delta \omega)$, depends on the central wavelength ( $\lambda=800 \mathrm{~nm})$ and the quality factor of the beam $\left(M_{2}=2\right)$, on the radius of the beam at the Fresnel hologram plane $\left(\omega_{0}=2.16 \mathrm{~mm}\right)$, and finally by the focal length difference among planes (in this case $\Delta f=50 \mathrm{~mm}$ ). By using this expression, based on the first measurement, the expected increment on the size of the foci for the other planes have been calculated ( $\Delta \omega \simeq 12 \mu \mathrm{m}$ ). Finally, this value indicates that the expected sizes on the other planes (150 $\mathrm{mm}$ and $200 \mathrm{~mm}$ ) are $45 \mu \mathrm{m}$ and $57 \mu \mathrm{m}$, which highly agrees with the measured values (48 $\mu \mathrm{m}$ and $59 \mu \mathrm{m})$. To sum up, 70 spots are generated in three different axial planes with high accuracy and uniformity. Although the results are positive, some minor variations between the theoretical and the experimental results are found. These variations can be caused by several reasons: the incident beam is not quite uniform and the optical system can introduce unwanted aberrations. In addition, the SLM can also introduce some error due to the problems discussed in chapter 2: pixelated surface, pixel cross-talk, etc. Finally, the recording device has also a pixelated structure, leading to an additional source of inaccuracies.

The aim of the second experiment is to demonstrate the ability of the method in certain applications. Particularly, it could be employed on laser material processing or non-linear microscopy. As an initial proof-of-concept, the purpose of this experiment is to generate non-linear effects in a suitable material. To perform it, the setup shown previously on Fig. V.4 is slightly modified to include a second $4 \mathrm{f}$ imaging system to reduce the size of the reconstructed complex field to a micrometric scale. Specifically, an additional 
lens $\left(L_{3}, f=400 \mathrm{~mm}\right)$ and two microscope objectives $\left(M O_{1}\right.$ and $\left.M O_{2}\right)$ are placed after the output plane of the first imaging system, where the complex Fresnel hologram is reconstructed. To do that, a phase pattern is encoded into the SLM again. Nevertheless, in this case, all lenses are designed with the same focal length, so in this time all foci are generated at the same axial position. This position is taken as the input plane of the second imaging system, which is formed by lens $L_{3}$ and a 20x microscope objective $\left(M O_{1}\right)$, and leads to a final demagnification of the beam of $1 / 73$. At the output plane of the system, a Type $1 \beta-\mathrm{BaB}_{2} \mathrm{O}_{4}$ (BBO) crystal is placed. A second microscope objective ( $\mathrm{MO}_{2}, 50 \mathrm{x}$ ) is placed after the BBO to collect all light: second-harmonic generated and fundamental light. In order to filter the non-converted light, a blue filter is introduced into the setup just before the CCD camera. Additionally, the bandpass filter employed on the previous experiment to reduce the bandwidth of the laser is removed. The new setup can be seen in Fig. V.6.

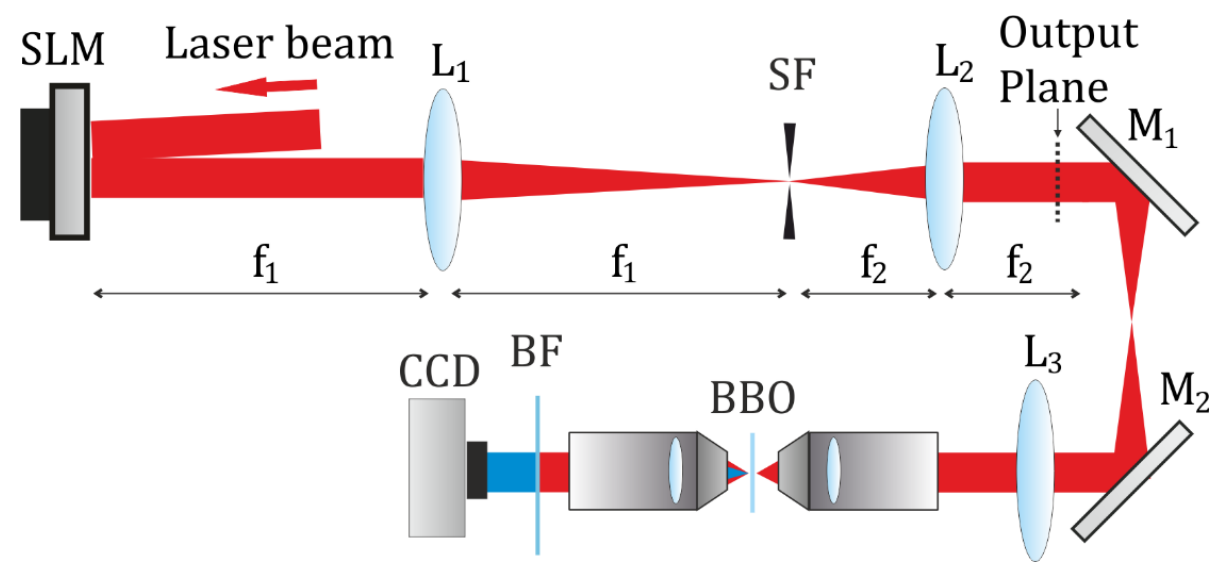

Fig. V.6 - Optical setup used to generate an arbitrary distribution of points over a non-linear crystal. Wavelength of recorded light is centered on $400 \mathrm{~nm}$. SF: Spatial filter. L1 - L3: lenses. M1 and M2: mirrors. BBO: non-linear crystal. BF: blue filter.

As it was mentioned above, all foci are generated at the same plane. The main reason is because in this experiment we wanted to show a fundamental arbitrary pattern and then, thanks to the BBO crystal, the same pattern but with second-harmonic light. To do it, the same procedure explained and used in the first experiment was employed to generate a 30 foci distribution similar to the structure of a common neuron. The generated foci distribution is 
displayed at Fig. V.7.a. Note that, in this image, fundamental light (800 nm) and not second-harmonic is shown.

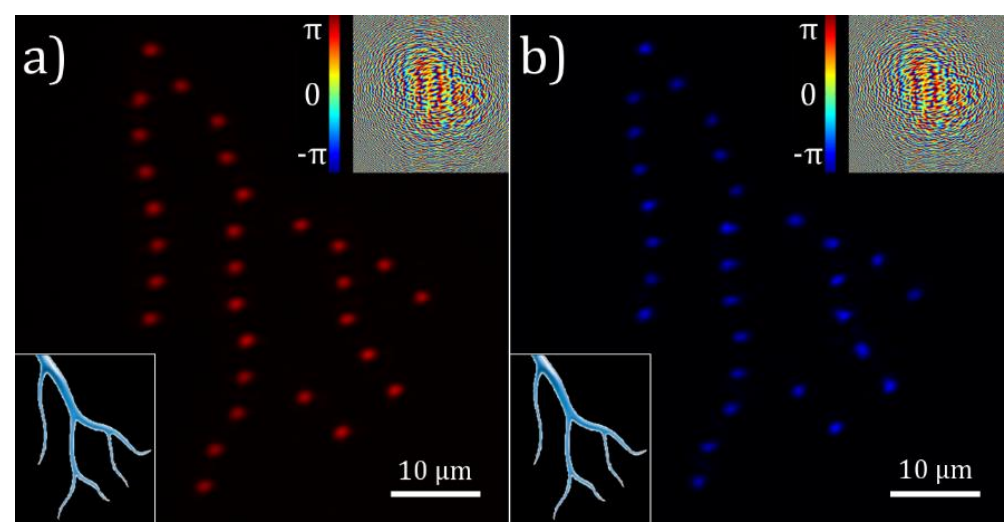

Fig. V.7-Images obtained for the second experiment. a) shows the obtained distribution using fundamental light. b) shows the same distribution using second-harmonic light, after placing a BBO crystal.

In the left-bottom part of both images, the original neuron image is included as a sample of how the foci distribution should be. In the top-right part, the alpha mask encoded into the SLM is also incorporated. In line with the abovementioned results, the foci that make up this distribution have relative intensity discrepancies less than $20 \%$ and transversal displacements below three pixels.

In the next step, a BBO crystal (0.01 mm thickness) has been added over the showed distribution of foci, generating the light distribution shown in Fig. V.7.b. In this case, the second-harmonic light depends on the square of the incident light and, therefore, the intensity variations are increased. In particular, the maximum irradiance disparity is now 30\% compared to the previous $20 \%$. In addition, other circumstances can help to raise this value; for instance, the different phase matching conditions related to the generation of the foci or the use of a laser with a large amount of spectral components. On the other hand, the positions of the foci are not affected by the BBO crystal, and show a similar behavior as in the previous experiment. Finally, size of all the foci following the $1 / e^{2}$ criterion are less than $2 \mu \mathrm{m}$, and all of them are contained within a transversal region of $55 x 55 \mu \mathrm{m}$, which is quite similar to the size of a large amount of cells. (rat hepatocytes $\approx 20 \mu \mathrm{m}$ [151], skin cells diameter $\approx 30 \mu \mathrm{m}$ [152], etc.). 
This proof-of-concept experiment shows that this method could be used at the illumination path of a non-linear microscope used to excite specific micrometric regions of a suited biological sample. One relevant point of this method is the ability to encode as many as foci as the available energy allows. This is, the number of foci is not restricted by the encoding method or the lens generation function. Moreover, their positions are also not limited by the technique, as two spots can be as close as one wishes. The only limitation is the interference produced by two overlapping foci. However, in other methods, an increase on the number of lenses entails a reduction of their radius with the consequences associated with it.

\section{V.4 - Micrometric complex illumination patterns for non-linear microscopy applications}

Our second proposal was published in 2019 and includes several different aspects [153]. The main idea is the use of the complex field encoding technique to generate an arbitrary amplitude and phase distribution able to trigger nonlinear effects. The intensity pattern reconstructed by the presented technique has been demonstrated as more uniform, in comparison with other wide-field techniques as CGH or IFTA-based algorithms. As previously stated, there is a quadratic dependency between the incident light and the non-linear excitation. Therefore, the uniformity of the irradiance pattern is extremely important to obtain a high-quality image based on the non-linear signal. In that vein, a proof-of-concept experiment was carried out to show the capabilities of this idea, demonstrating both single-photon and second-harmonic generation (SHG) with structured illumination over large-area regions of interest (ROIs). Additionally, other experiments were performed to demonstrate the usefulness of controlling not only the irradiance but also the phase at the sample plane.

Specifically four experiments were completed. The first one was realized to show the ability of the method to generate a high-uniformity long-region irradiance pattern, the ability to encode a multi-level gray image, and finally to define the region of interest for all experiments. For that reason, only singlephoton images were recorded in this first step. The second experiment was aimed to show the ability of generating second-harmonic light on a large region. In comparison with the previous manuscript, where SHG was carried out on an arbitrary selection of spots, here it is performed on the whole ROI simultaneously. The purpose of the final two experiments is to show the possibilities unlocked by the phase control. First, it has been used to 
compensate for possible aberrations introduced by the optical system or the beam itself. Secondly, we exploit the encoding technique to obtain an interference pattern generated by the illumination and the sample. In that way, it is possible to use the reported method to extract useful information about the sample.

In all four experiments, the employed setup is very similar, although there are some minor differences among them. For instance, for the SGH experiment, a second-harmonic crystal is employed, which is not needed on the single photon experiment. The setup is basically formed by two 4 f imaging systems, and is quite similar to the one shown in Fig. V.6. Lenses $L_{1}$ and $L_{2}$ form the first $4 \mathrm{f}$ imaging optical system, which objective is to generate the arbitrary complex field at the output plane of the imaging system. As in previous setups, the SLM is placed at the input plane, whereas at the Fourier plane, a spatial filter is required to filter all diffraction orders but the zeroth one and to coherent sum the two phase terms resulting on the double phase equations. The second imaging system is employed to arbitrarily demagnify the size of the pattern to fit the sample or the selected ROI. It is formed by lens $L_{3}$ and a 20x microscope objective $\left(M O_{1}\right)$, and forms a reduced image of the generated complex field (input plane) over the sample plane (output plane). Depending on the requirements of the experiment, additional elements would be placed at the sample plane. For instance, in our second test, a BBO crystal is placed to trigger SHG. Finally, a second microscope objective $\left(50 \mathrm{x}, \mathrm{MO}_{2}\right)$ is used to form an image of the sample plane over a CCD. In the non-linear experiments, a band-pass filter is placed after the BBO to let pass by the second-harmonic light and filter the fundamental one. The setup is shown in Fig. V.8. 


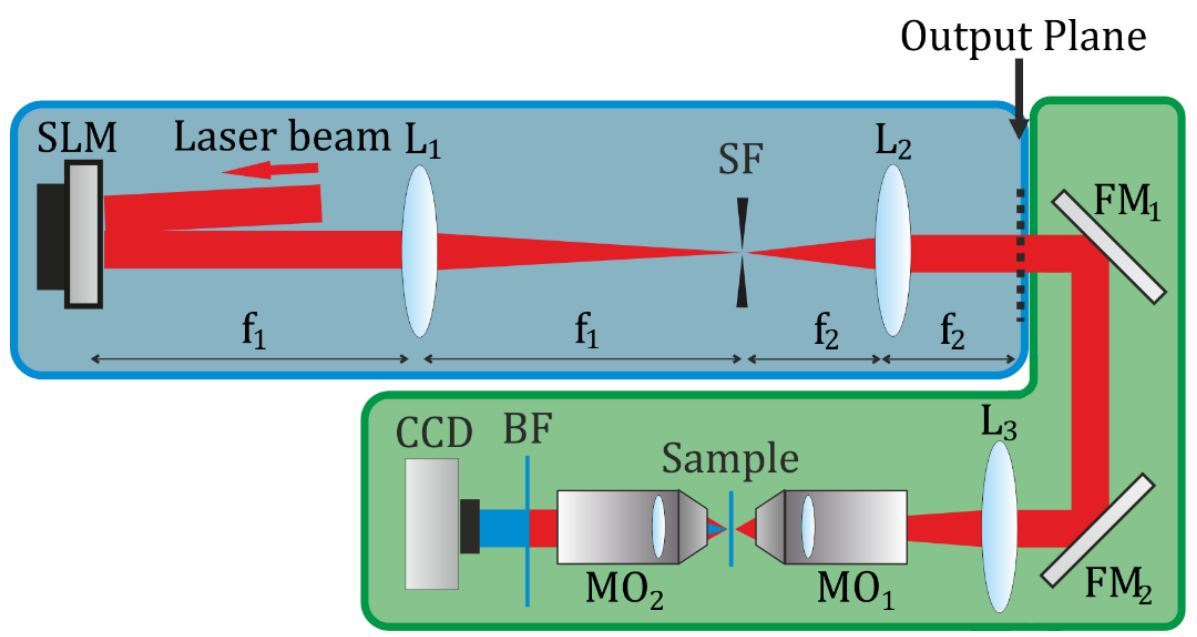

Fig. V.8-Optical setup employed to obtain the desired complex field using the proposed method. The blue area is a 4 f imaging system used to generate the complex field at the PhPx plane. The green region is another $4 f$ imaging system employed to reduce the size of the pattern to the microscopic scale. L1

- L3: Lenses. SF: Spatial filter. FM1 - FM2: Flip mirrors. MO1 - MO2: Microscope objectives. BF: Blue filter.

The image clearly distinguishes the two imaging systems. The blueish part is the one addressed to generate the arbitrary complex field. This part is formed by several optical elements, including $L_{1}$ and $L_{2}$. As the focal lengths of these elements are $\mathrm{f}\left(L_{1}\right)=1000 \mathrm{~mm}$ and $f\left(L_{2}\right)=500 \mathrm{~mm}$, the complex field $U(x, y)$ reconstructed at the output plane of this system has a transversal magnification of $1 / 2$. On the other hand, the green background shows the demagnifying section. The magnification of this part is given by the optical elements $L_{3}$ and $M O_{1}$, but these elements can be exchanged depending on the requirements of the experiment, in particular: the size of the examined sample. In our case, the focal length of $L_{3}$ is $400 \mathrm{~mm}$. Finally, other elements are employed within the setup but are not included in the figure. This is the case, for instance, of neutral filters, which were used to reduce the intensity of the light, avoiding harmful effects over the SLM surface or over the sample. Note that, the final demagnification of the system is $1 / 73$, which means that all final energy is confined into a micrometric region.

As discussed below, another method has also been used to compare the quality of our technique. In this case, an IFTA-based CGH was employed to assess the performance of the reported method in comparison with a well- 
stablished technique. For that reason, the experimental setup was slightly modified when the CGH experiments were carried out. These modifications can be observed in Fig. V.9.

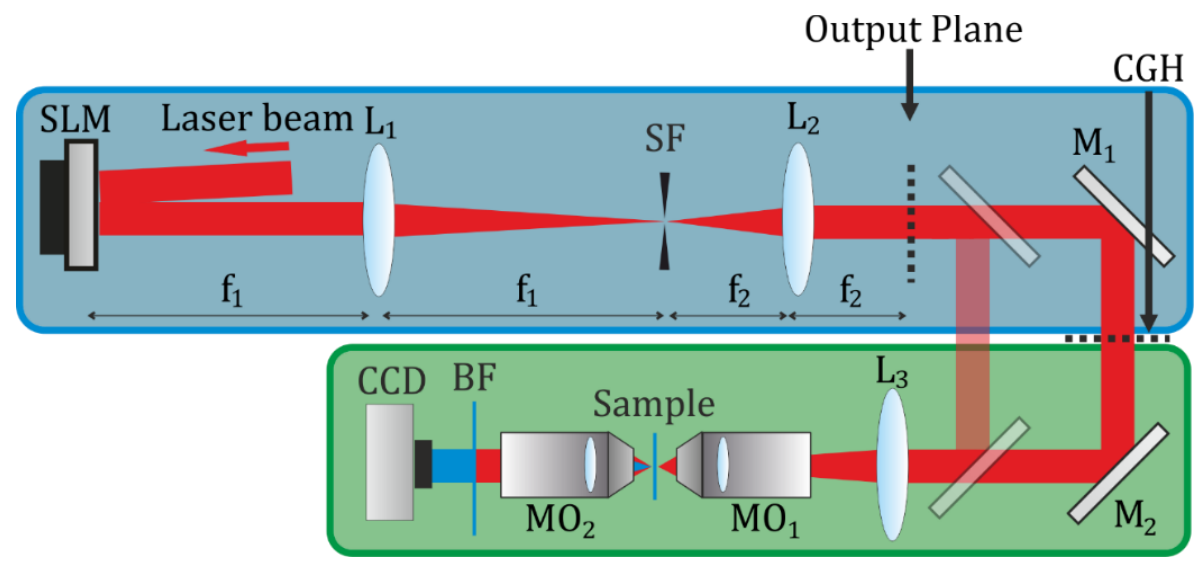

Fig. V.9-Optical setup employed to obtain the complex field using a hologram generation technique. The blue area here reconstructs a hologram over the CGH plane. The green region accomplish the same objective as in the previous setup. The shaded elements represent the elements removed from the system with respect to the previous one. L1 - L3: lenses. SF: Spatial filter.

M1 - M2: Mirrors. MO1-MO2: Microscope objectives. BF: Blue filter.

The flip mirrors (now shaded) were removed from the system (flipped) allowing the light to pass by. In this way, light impinges onto the mirrors ( $M_{1}$ and $\boldsymbol{M}_{2}$ ) increasing the optical path of the setup. Note that, the $\mathrm{CGH}$ is reconstructed at a certain distance after the output plane of the first imaging system. Due to the addition of this delay line, the distance between the CGH and $L_{3}$ is the same than the previous distance from $L_{3}$ to the complex field $U(x, y)$, maintaining the magnification of the system.

The selection of the optical components on the magnifying system is essential to achieve a rough arrangement of the illumination pattern. However, the fine adjustment is performed with the SLM, not only to shape the beam, but also to reduce the size to precisely fit the sample. For that reason, the first step should be the selection of the ROI, and only after that, the complex field $U(x, y)$ can be defined.

In our case, an exemplar of a Daphnia was chosen for this purpose. In Fig. V.10, an image of the selected specimen taken in a conventional microscope is seen. 


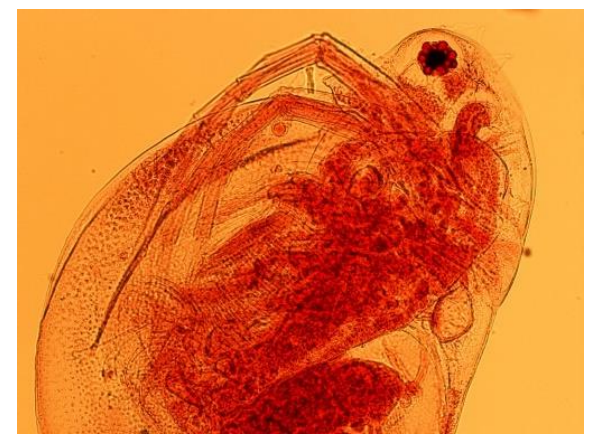

Fig. V.10 - Image of the specimen employed to select the ROI.

However, as one can see at the scale bar, the size of the sample is a little bit large, reaching almost the micrometric scale. As our method is capable of generate a micrometric complex field and this experiment tries to push the limits of it, we have adapted by choosing just the eye of this exemplar (Fig. V.11.a) and selecting a ROI that fits this area (Fig. V.11.b). Once the shape of the pattern is defined, it is possible to start the experiments.

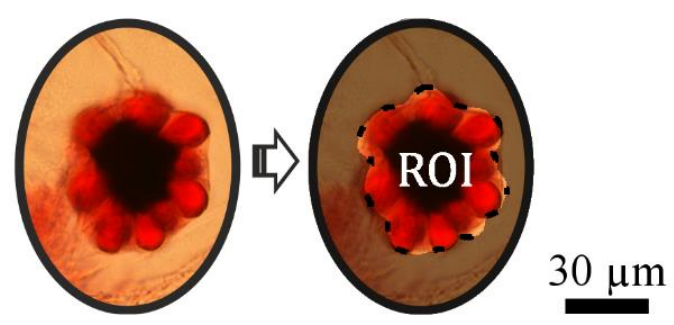

Fig. V.11 - Left part: zoom at the eye of the Daphnia. Right part: the brightest part shows the selected ROI for the experiments.

To start with, a single photon experiment was performed. The main objective of it is to show briefly the precision of the arbitrary irradiance pattern generated. In that way, a high quality shaped illumination could be employed in a microscope, but also to trigger non-linear effects over an extended area. In this regard, the uniformity of the pattern is extremely important due to the quadratic dependence between the incoming irradiance and the output intensity. An irregular original pattern leads to a very uneven harmonic second signal. An example of this will be shown later. In addition to the generation of the arbitrary irradiance pattern, the ability to manipulate at will the phase within this pattern is also shown. 


\section{Theory}
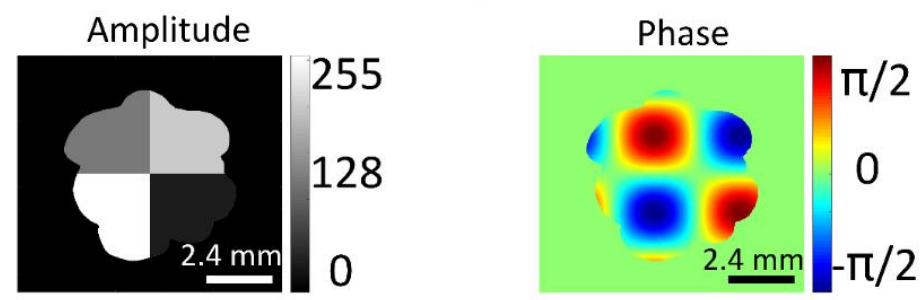

\section{Experiment}
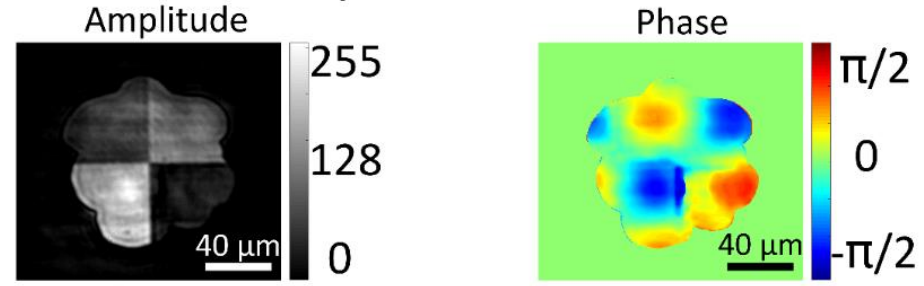

Fig. V.12 - Example of an encoded complex field (top part) and the recorded amplitude and phase for the generated phase mask (bottom part).

Fig. V.12 shows the result of this test. In the top part of the figure (named as theory), both the amplitude and phase of the desired complex field is shown. The amplitude pattern is shaped as the ROI defined previously. Moreover, it is composed by four different energy levels distributed among four similar size quadrants inside the ROI. This intends to show not only the ability to shape at will the pattern, but also the possibility of spatially control the irradiance or the "gray-levels" within the beam. The phase is defined by an arbitrary function. Actually, any phase could have been chosen but in this case, it is given by the analytical function $\varphi(x, y)=\pi / 2 \sin [2 \pi x /(5 \lambda)] \cos [2 \pi y /(5 \lambda)]$.

The recorded experimental complex field is shown in the low part of Fig. V.12 (named as experiment). Note that according the scale bar, the selected ROI dimensions are about $60 \times 60 \mathrm{~mm}^{2}$. In the upper image of Fig. V.12, the scale bar represents the size of the pattern at the SLM plane. Both experimental amplitude and phase results quite agree with the theoretical ones, however small discrepancies are found. In particular, the root-mean-square error (RMSE), which is obtained from the square root of the mean squared difference between the expected values and the measured ones, yields results of $6.8 \%$ for the amplitude and $14 \%$ for the phase measurement. Several aspects contribute to increase this error. First, the transversal profile of the 
employed laser beam irradiance is not homogenous. Secondly, as one can see in the experimental setup (Fig. V.9), this laser beam is not impinging the SLM parallel to the normal incidence, but forming a small angle instead (about 3 degrees). Finally, and related with the encoding technique, the spatial filter placed at the Fourier plane of the first imaging system slightly reduces the spatial resolution. The reason is that at the output plane of the imaging system, instead of the complex field, we can find the convolution of the complex field (reduced and spatially reversed) with the Fourier transform of the spatial filter.

To record these images (Fig. V.12 - experiment) a polarization-based phase shifting technique [118] was employed. This technique allows reconstructing amplitude and phase at the sample plane by the measurement of four interferograms but requires the use of additional optical elements. Specifically, two polarizers are enough to achieve it. The first one is a halfwave plate employed to rotate the original direction of polarization before the SLM. To correct align it, it should rotate all frequency components 45으 with respect to the SLM director orientation. A broadband halfwave plate is recommended due to that reason. The SLM only modulates incident light polarized parallel to its director orientation. By rotating the beam 45을 we ensure that about half of the light is being diffracted by the SLM while the other half is not. As mentioned previously, this technique is based on the measurement of four interferograms, and these are generated by the interference of the diffracted (object beam) and the non-diffracted light (reference beam). Taking advantage of the SLM, uniform phases with equidistant steps between them are to the phase element $\alpha$. In that way, for the first interferogram $\boldsymbol{I}_{1}$, no phase is added, but for the other three $\left(\boldsymbol{I}_{2}, \boldsymbol{I}_{3}\right.$, and $\left.\boldsymbol{I}_{4}\right)$, phases $\pi / 2, \pi$, and $3 \pi / 2$ are added, respectively. To generate the interference between the object and the reference beams, a second polarizer is used. In this case, a mere broadband linear polarizer is needed after the lens $L_{3}$ to recombine both beams. At this point, the diffracted and non-diffracted beams should propagate with perpendicular polarization directions. This polarizer regulates the amount of light that passes through it. In order to optimize the interferogram, the proper position of the polarizer should be 450 with respect to both beams so that they have similar intensity after it. As an example, the four interferograms recorded for these experimental results are shown in Fig. V.13. 


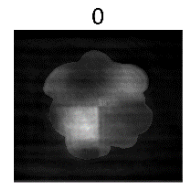

Phase-shifted interferograms
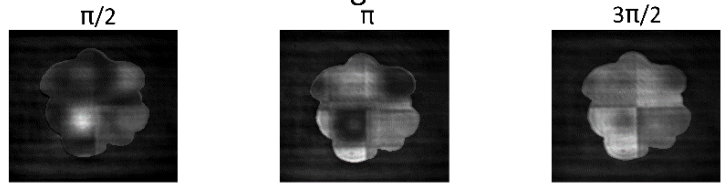

Fig. V.13 - Four interferograms recorded by using the phase shifting technique. Above each image, the phase shift added by the SLM is displayed.

This procedure has demonstrated the ability to generate an arbitrary amplitude and phase complex field. The phase pattern could range continuously from $-\pi$ to $\pi$. This means that a wide series of different phases can be found in the image. On the other hand, the amplitude pattern is made up of only four different energy levels or "gray-levels" although the technique theoretically allows up to 256. In order to test that, a second single photon test was carried out, but this time with a flat phase. Following with the ROI defined previously, a gray levels image of a mushroom with the silhouette of the ROI was encoded into the SLM. In addition, as one of the most commonly demanded patterns for certain applications is a uniform pattern, it was also performed a test with a flat amplitude pattern. Both results are shown in Fig. V.14.

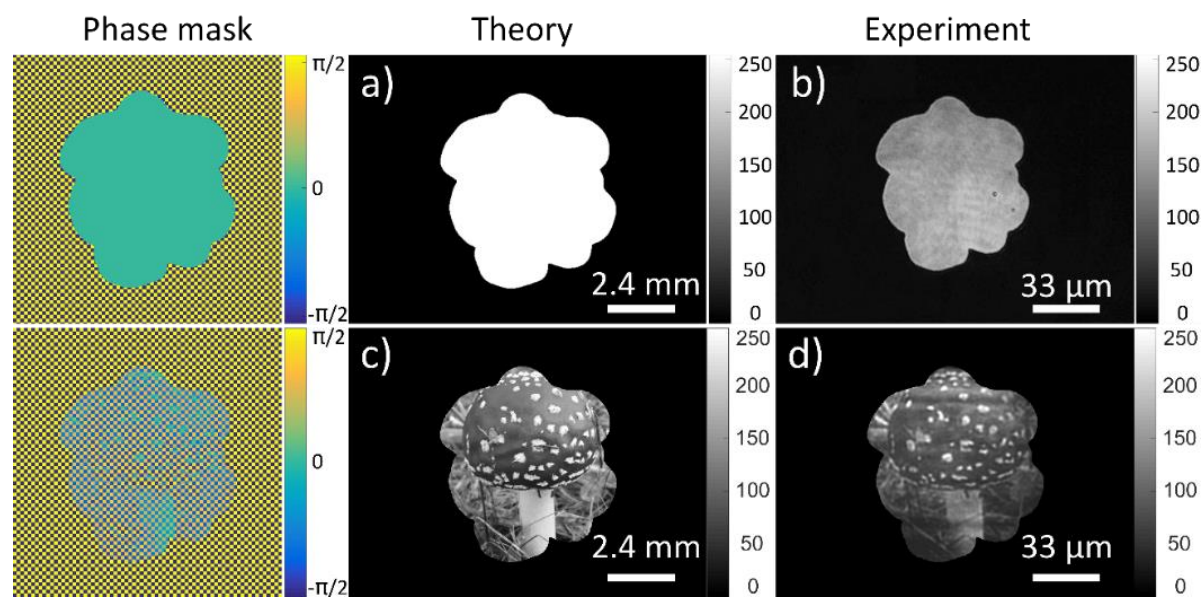

Fig. V.14 - Example of a couple of desired (central column) and obtained (right column) amplitude patterns. The left column displays the $\alpha$ mask employed to generate the pattern. The top row shows a uniform pattern using a single intensity level whereas the bottom row employs 256. 
The first row shows the irradiance pattern that we want to obtain at the sample plane (left) and the one we recorded with our experimental system (right). This pattern corresponds to the shape of the Daphnia's eye with a uniform intensity. At a first glance, it seems that both images are very similar because the experimental image has high contrast, a well-defined edge, and quite uniform filling. The RMSE confirms that, yielding a 7\% difference among them. However, this pattern is free from coherent noise since it is constructed by conjugating the SLM plane with the sample plane, so other issues cause these discrepancies. Specifically, the main sources of the error are aberrations caused by the experimental setup (dust found on the surface of the optical elements, slight misalignments, etc.) and the irradiance profile of the illumination at the SLM plane, as non-uniformities at this plane will result in non-uniformities at the sample plane. The second row tries to implement a 256 gray-level image that requires full control over the reconstructed amplitude at the sample plane. As an example, the ROI shape resulting in the image shown in Fig. V.14.c has trimmed the image of a mushroom. In Fig. V.14.d one can see the recorded image. In this case, the reconstruction is moderately weaker than the previous example, as the RMSE parameter indicates (10\%), which is still a very acceptable result. Note that the size of this image is about $60 x 60 \mu^{2}$, so the system is able to resolve very small details as the white dots present in the cap of the mushroom (about $5 \mu \mathrm{m}$ ). Taking in account the pixel size of the SLM $(8 \mu \mathrm{m})$ and the magnification of our system, the minimum feature size that is able to resolve is $0.87 \mu \mathrm{m}$. To sum up, both results reach a desirable result. The first one could be easily employed on any system that only cares about a single level of excitation whereas the second one open new paths to some useful applications on different fields. For instance, it allows performing simultaneous micro-processing of different materials with diverse ablation thresholds, or also allows to excite non-linear effects over several fluorophores with different excitation thresholds.

In the next step, the non-linear experiment was performed. Basically, it is just a proof-of-concept test to show non-linear excitation using the reported method. The main purpose is to generate second-harmonic light in a controlled region defined by the ROI employed in all the experiments shown here. In this way, the irradiance pattern is the one shown in Fig. V.14.a, that is, a uniform irradiance pattern with the shape of the ROI.

In left part of Fig. V.15, we show the experimental results of generating the desired amplitude, which is (obviously) quite similar to the result shown in Fig. 
V.14.b. In addition, at the right part, the second-harmonic signal generated using this pattern is shown.

Fundamental illumination pattern
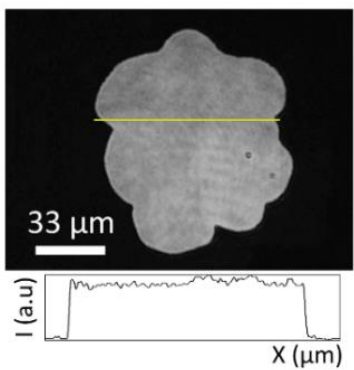

\section{Measured pattern with SHG light}

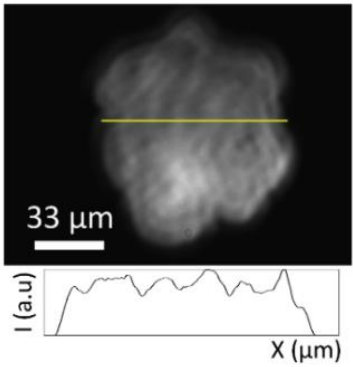

Fig. V.15 - Example of a SH image (right) generated by a BBO crystal illuminated by the irradiance pattern showed at the left part of the image, and generated using the proposed technique. Below the images, the intensity profiles measured along the yellow lines are included.

The image is almost fully flat and free from speckle noise, however, as one might expect, the acquired SHG pattern is slightly worse than the fundamental result. This occurs because of the non-linear crystal amplifying the nonuniformities of the incoming light. To better illustrate the uniformity of both patterns, the irradiance profile is included at the bottom part of each image. These irradiance profiles shows the measured intensity level along the yellow lines displayed more or less at the central part of the ROIs. In this case, the RMSE along these profiles yields a $12 \%$ error on the fundamental pattern and $23 \%$ with SHG.

We think that our method shines when compared to other techniques used to generate amplitude illumination patterns. With this purpose on mind, the same measurement was performed but this time with a CGH synthetized by a Gerchberg-Saxton IFTA-based algorithm [18]. As the algorithm, needs a certain propagation distance to achieve the reconstruction, a delay line was included in the setup to control distance between the second imaging system and the reconstructed $\mathrm{CGH}$. In this way, the IFTA algorithm has enough space to obtain the hologram while the magnification of the system is not affected. This setup is shown in Fig. V.9. The design of the holograms was made following a twostep procedure. In the first step, the phase was the only free parameter during the iterations performed by the IFTA algorithm. Giving full freedom to the phase results in a better approximation. However, as the SLM is a digital device 
that can only encode a certain number of phase levels, a soft restriction is applied in the second step to limit the amount of phase levels that make up the hologram. As all IFTA-based algorithms, a convergence threshold should be defined to stop the execution of the program. In this case, the execution finishes when the RMSE resulting of an iteration enhance the previous result by less than $10^{-8}$. The recorded hologram and the second-harmonic image obtained with the CGH illumination are shown in Fig. V.16.

\section{Fundamental illumination pattern}

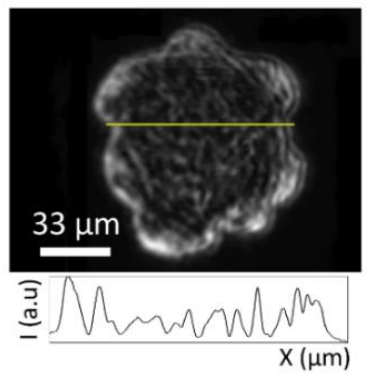

\section{Measured pattern with SHG light}

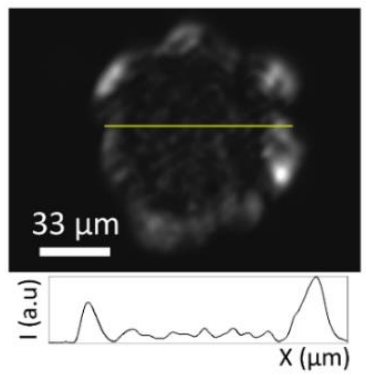

Fig. V.16 - Example of a SH image (right) generated by a BBO crystal illuminated by the irradiance pattern showed at the left part of the image, and generated with a standard CGH algorithm. Below the images, the intensity profiles measured along the yellow lines are included.

At first sight, both results are clearly worse than the ones obtained using the proposed technique. The hologram resembles the defined ROI but is far from being uniform. The most noteworthy feature is that there has been a clear reinforcement of the edges, but the coherent noise prevents the filling to be free from speckle. The second-harmonic image is still worse due to the mentioned quadratic dependence between the incident light and the SHG. In this case, not only the borders are reinforced, also the central part shows almost no light. The transversal profiles are also included in these images, displaying the irradiance measured along the yellow line. These profiles confirm that the result obtained with our technique is better than with the CGHs. If the RMSEs previously measured between the profiles shown and a flat profile was $12 \%$ and $23 \%$ for the single-photon and SHG, respectively, the RMSEs calculated here are $62 \%$ and $77 \%$ (single-photon and SHG). Just a naked-eye exploration is enough to determine that these latter profiles are noticeably irregular and, especially the second one lacks of intensity in the central part. 
Although only amplitude results are shown, the full complex field $U(x, y)$ was encoded into the $\alpha$ phase mask. In particular, the phase was selected to compensate the possible optical aberrations introduced by our system or by other means. For this, the phase was measured in the sample plane (after encoding a flat phase onto the SLM), conjugated and then introduced as the phase term of the complex field $U(x, y)$.

Finally, just comment that to obtain the second-harmonic images, a BBO crystal was placed at the output plane of the second $4 \mathrm{f}$ imaging system (shown in Fig. V.8 and 5.9). The dimensions of the crystal are $5 \mathrm{~mm} * 5 \mathrm{~mm} * 0.02 \mathrm{~mm}$, and after this crystal, a blue filter was placed to remove all light but that converted by the BBO.

Up to now, we have just briefly mentioned the control over the phase enabled by the proposed technique. However, it allows plenty of different possibilities. Probably, the most obvious may be the aberration correction. The optical elements that make up the setup or the laser source itself may introduce some unwanted phase aberrations that can be potentially harmful in some applications. Just measuring the phase profile at the sample plane and adding a phase term to the generation of the complex field $U(x, y)$ is enough to correct them. To perform this measurement, the phase shifting technique [118] employed in other experiments is useful here too. However, in this case, a two-arm interferometer (Michelson type) is designed and implemented instead of the one-arm employed before. This type of interferometer allows the detection of the optical aberrations introduced by the optical system. Modified setup is shown in Fig. V.17.

The most noticeable difference is the addition of a broadband beam splitter that divides the beam into the mentioned two arms. The object beam is sent from the beam splitter to the SLM, reflected back and reaches again the beam splitter, but this time passing through it. At the same time, the reference beam is reflected by a silver mirror placed at the same distance of the beam splitter as the SLM. The silver mirror is placed over a precise micrometric displacer to adjust the position to match both optical paths. Note also that in this setup, the laser beam impinges perpendicular to the LC display. This setup is developed to complete a proof-of-concept test. Here, the interferometer is able to measure aberrations introduced by the SLM, as this is the only element present on the test path not found on the reference path. In this way, once the aberrations are retrieved, it is possible to correct them by simply encoding the conjugate of this phase with the proposed method. 


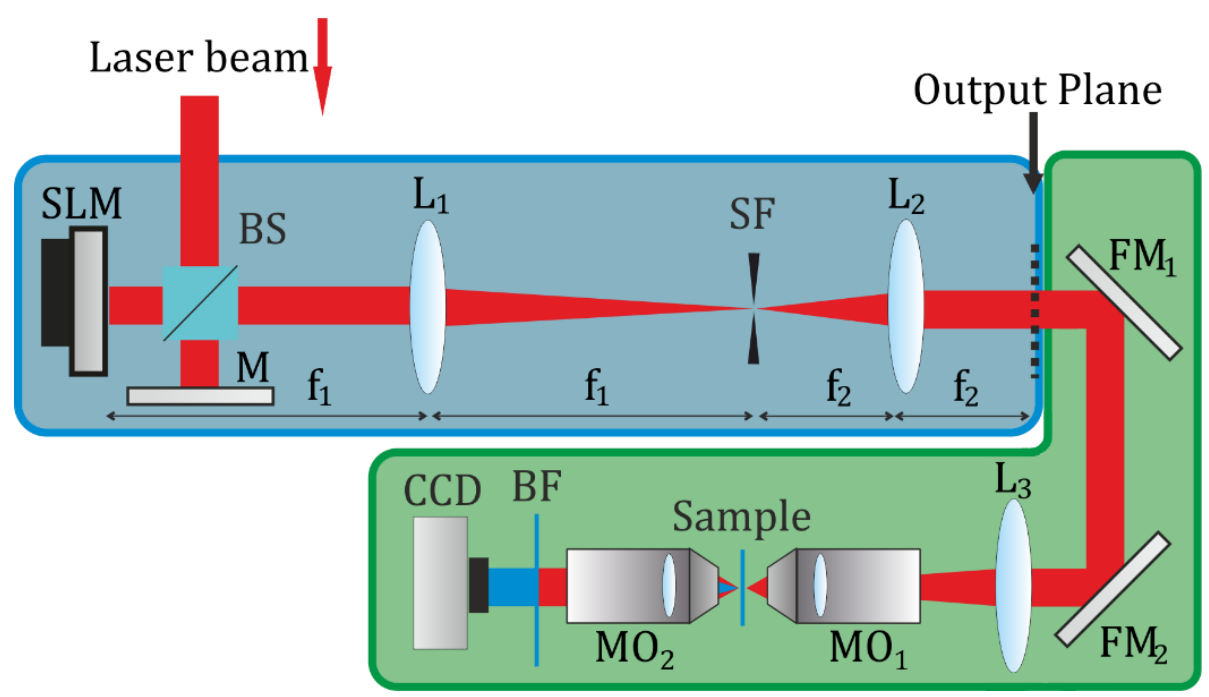

Fig. V.17-Optical setup including a Michelson type interferometer. Setup is designed to form the desired pattern over the sample using the proposed technique.

BS: Beam Splitter. M: Mirror. L1 - L3: Lenses. SF: Spatial filter. FM1 - FM2:

Flip mirrors. MO1 - MO2: Microscope objectives. BF: Blue filter.

In Fig. V.18 two examples performed with this arrangement are shown. The first one simply does what has just been said: measure the phase in the sample plane and correct it to obtain a flat profile. Fig. V.18.a shows the wanted phase, Fig. V.18.b shows the measurement without correction, and after the correction, we obtain the pattern shown in Fig. V.18.c. The second one is more complex: the objective is to correct these aberrations while encoding an arbitrary phase pattern. Prior to the measurement, the phase function displayed at Fig. V.18.d was encoded into the SLM. After that, without phase correction, the phase recorded presents some unwanted aberrations (Fig. V.18.e). Finally, after the proper adjustment, the final measured phase is shown (Fig. V.18.f). The amplitude pattern is the one employed in previous experiments. It is shown at the bottom-left part of the images of the first column. In addition, the retrieved amplitude image is also displayed at the bottom-left part of all four phase measurements.

This figure just indicates how important the phase correction is to better approximate the desired phase pattern. Additionally, it also shows the possibilities that our method offers, being able of encoding a phase function and correcting the aberrations simultaneously. 

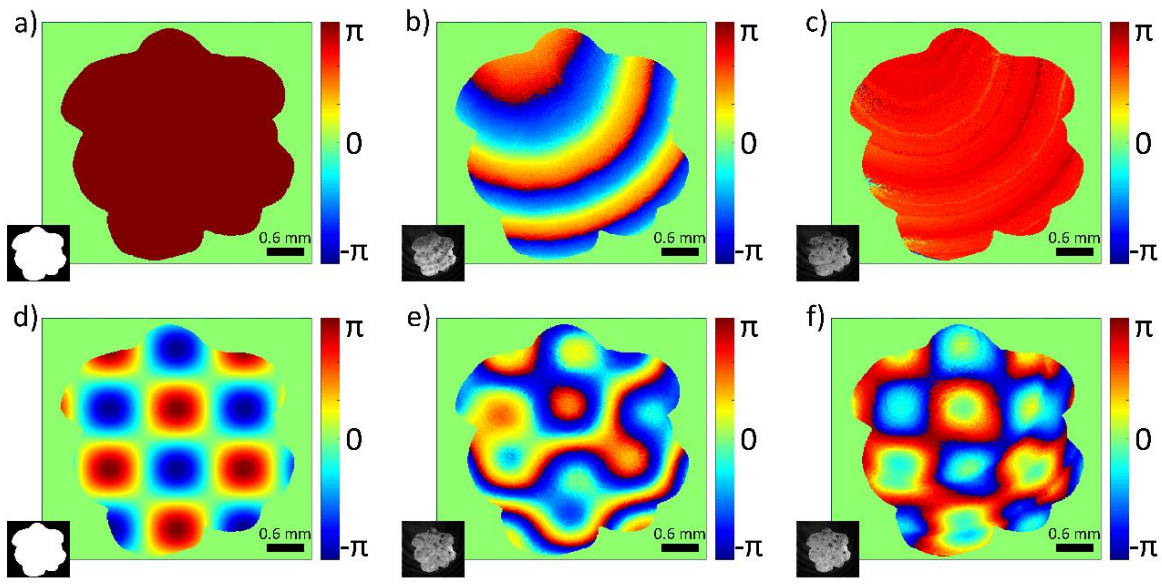

Fig. V.18-Example of phase compensation. Top row (a-c) displays a desired uniform phase pattern (a), the measured phase without compensation (b), and the measured phase after compensation (c). Bottom row ( $d-f$ ) displays an arbitrary phase pattern (d), the measurement before compensation (e), and after compensation (f).

The fourth and last experiment is aimed to demonstrate some applications of the phase control. For instance, in non-linear excitation of large area ROIs, the possibility of arbitrarily modify the phase can enable the gathering of additional information about the sample. With that on mind, we designed an experiment where two adjacent ROls were defined to excite simultaneously second-harmonic signal. Then, after a certain defocusing distance, an interference pattern produced by the signals that come from the two ROls is recorded [154]. The purpose of this experiment is to analyze and demonstrate the dependence on the phase shift of the ROls over the interference pattern, and then claim the utility of the reported method to generate the interference patterns at will due to the possibility of independently control both amplitude and phase.

The experiment results are shown in Fig. V.19. Here, four images were recorded for four different phase shifts between the two ROls. At the top-right part of the image, small representations show the shape and the phase of both ROls. The green color represents a phase value of 0 , whereas the yellow, orange, and red represent $\pi / 2, \pi$, and $3 \pi / 2$, respectively. The inner ROI remains green in all pictures, while the outer one varies with $\pi / 2$ steps. This means that the phase shift in Fig. V.19.a between both ROIs is 0, in Fig. V.19.b is $\pi / 2$, in Fig 5.19.c is $\pi$, and in the last one is $3 \pi / 2$. Although here only four 
different phase shifts have been recorded, the technique allows encoding a much wider range, only restricted by the gray levels addressed to the display of the SLM.

a)

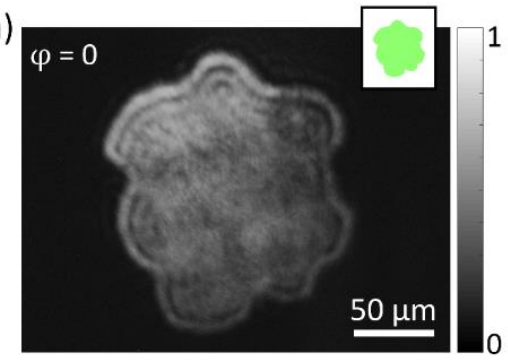

c)

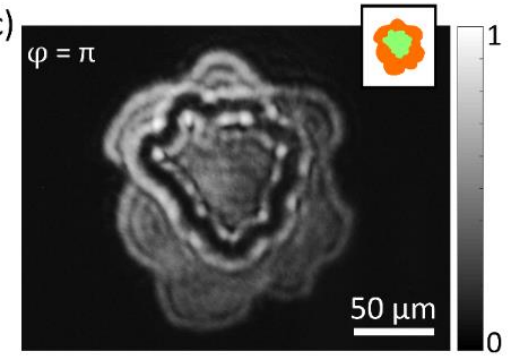

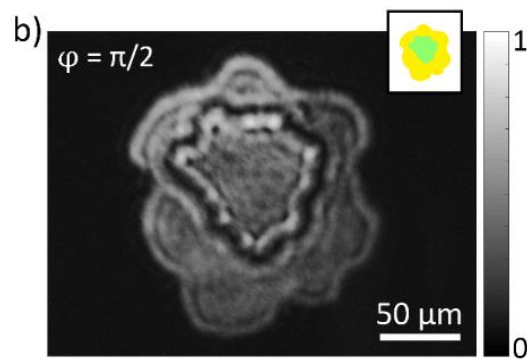

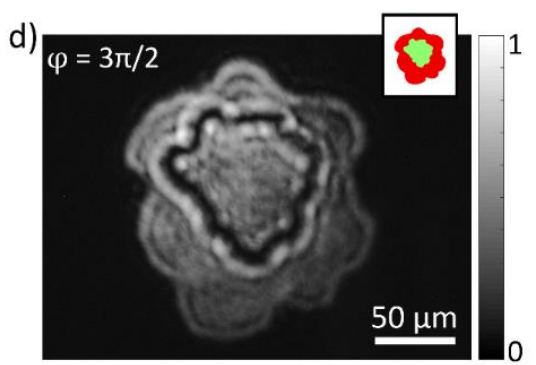

Fig. V.19-Phase-dependent measured interferences. Four different images were recorded with four different phase shifts between two ROIs. Phase shifts are displayed at the upper-left part of each image. ROIs are included at the top-right corners.

As it might be expected, Fig. V.19.a shows only a defocused image of the amplitude pattern generated by the addition of the two ROIs and no interference between them. This is typical since both ROIs have the same phase, so there is not phase shit between them. In practical, we are just encoding one ROI with a flat phase. Things change when the phase of the inner $\mathrm{ROI}$ is modified. The top-left part of the four images indicate the phase shift $\varphi$ among the two regions. The most relevant fact of this experiment is that, as one can see in Fig. V.19.b-d, the structure of the interference pattern depends on the phase shift between ROls. Obviously, the closer to the border between the two ROls, the greater the effect of the interference and, therefore, the greater the difference between images in that area. In this experiment, the camera was placed $115 \mathrm{~mm}$ away from the sample plane, letting the interference to happen, but not deforming significantly the shape of the ROls. 
At this point, interference between both ROls has been demonstrated. However, one may think that the results shown in Fig. V.19 are not caused by the interference effect that we claim, but by a border effect. In order to avoid it, the last experiment is aimed to ensure that the amplitude differences are undoubtedly produced by the interference between the two ROls and can be controlled with the phase shift. The main difference in comparison with the previous test is that there is now a small ring separating both ROIs. In that way, the interference pattern can only be generated by the propagation of both ROls and not by a border effect at the sample plane. The separation between the ROIs has an additional effect: the propagation distance needed to obtain the interference is increased. Then the defocused image does not fit the size of the CCD. To solve this issue, a convergent lens $(f=100 \mathrm{~mm})$ was placed after the reconstruction plane to adjust the image size over the CCD. The camera was placed in an arbitrary position where the image meet all size and propagation requirements.

A set of images were recorded with the same procedure as before: one ROI remains with an invariant phase whereas the other takes different values. Four of these images are shown in Fig. V.20. Following the same scheme as before, the inner ROI remains with a uniform phase taking the value zero while the outer region takes several different values. In the figure, we show the values 0 (Fig. V.20.a), $\pi / 2$ (Fig. V.20.b), $\pi$ (Fig. V.20.c), and $3 \pi / 2$ (Fig. V.20.d).

Fig. V.20 clearly shows the presence of interference fringes in the region comprised between both ROls. Now, these fringes cannot be generated by border effects so it demonstrates the interference due to the propagation. Moreover, as one might expect, this pattern is dependent on the phase shift. The top-left part of each image shows the phase shift encoded in this image. The top-right part shows the encoded pattern following the color rules described for the previous figure. The main conclusion extracted from this experiment is the possibility of employing this technique to control of the generated complex field over the sample plane for several applications: not only to correct aberrations, but also to extract phase information from a real sample (i.e. a biological one). 
a)
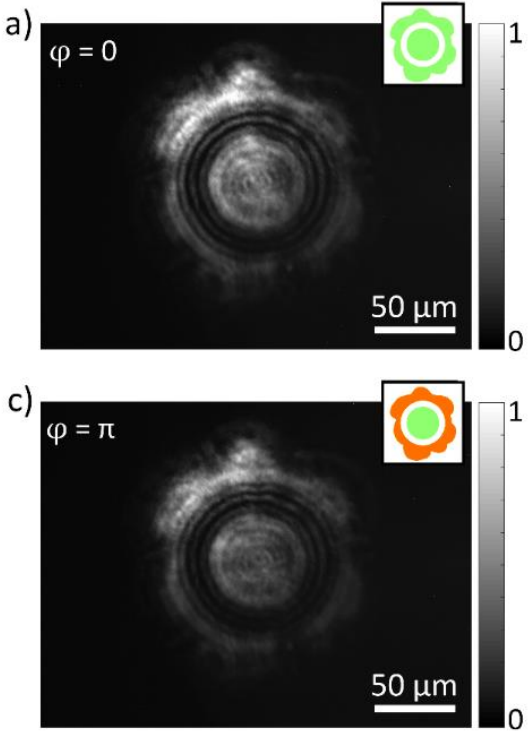
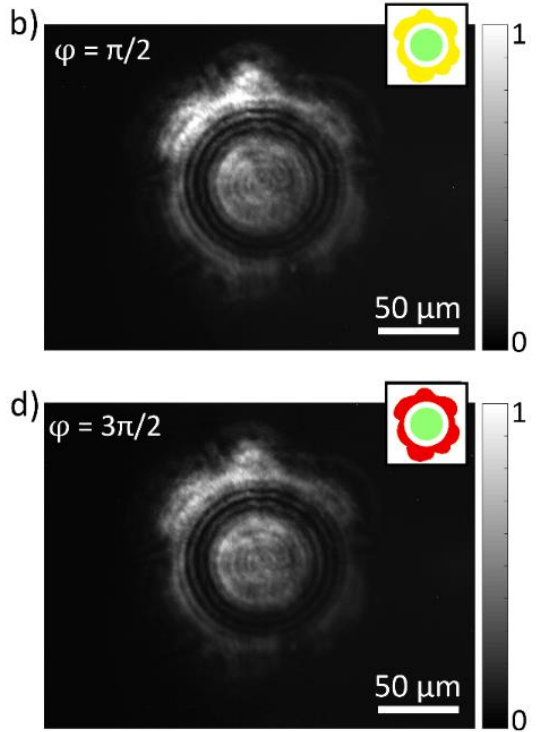

Fig. V.20 - Example of interference between two ROls spatially separated. Different phase shifts between ROls are employed for each image (displayed at the upper-left part of each image). ROls are also included at the top-right corners.

Once the experiments have been performed and reviewed, is time to extract some conclusions from them and make some considerations about the system. The first one should be the efficiency of the method, and the comparison with the one obtained with the IFTA-based CGH. This gives an idea of the position of our method with respect to other techniques. However, losses of energy are not only caused by the encoding method, but also by other different factors. For instance, beam splitters are a main source of energy reduction if they are employed to impinge the SLM perpendicular to the display surface. Otherwise, an obliquus orientation also entails a minor but also noticeable reduction. The SLM itself is probably the major origin of issues. The manufacturer usually notifies certain parameters related with this topic. For instance, the reflectivity indicates the ideal amount of light thrown back by the mirror of the SLM. In our case is 65-75\%; however, this parameter depends on different conditions as the angle of incidence mentioned just a few lines above or the employed wavelength. This basically means that if the wavelength of the laser beam employed doesn't fit the conditions for which the coating is prepared, then the reflectivity drops. Fig. V.21 and 5.22 shows the different versions of two different SLMs models. Each version of each 
model has the same chipset, communications interface, and LCD, offering thus the same spatial resolution but the mirror they use to reflect the light back (note that these are not transmissive SLM but reflective ones) are different. Most common mirrors are dielectric or aluminum, and the coating employed on them is different according to the version. In addition, this parameter affects on other characteristics of the SLM as the maximum incident power allowed, since a high reflectivity implies a lower absorption.

\section{PLUTO-2 Phase Modulator Versions}

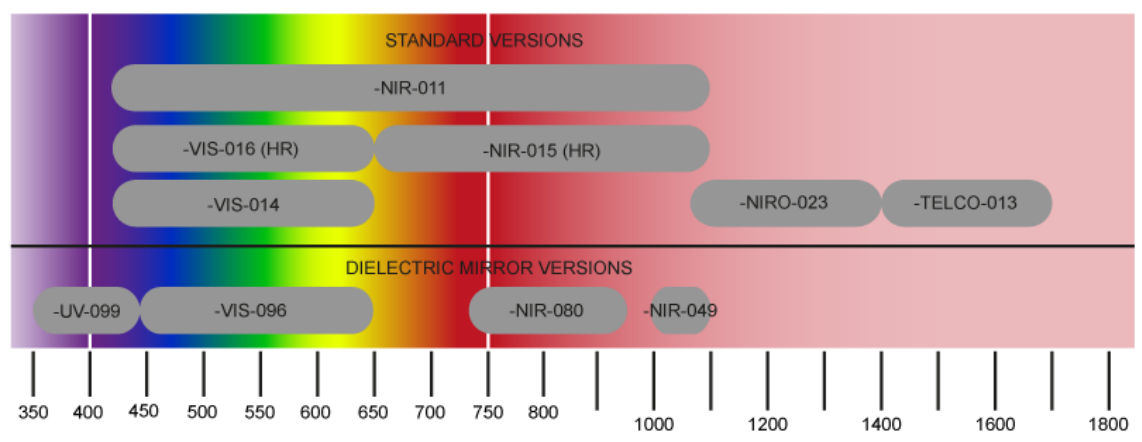

Fig. V.21 - Versions of the SLM Pluto-2 from Holoeye. Image obtained from [55].

I Hamamatsu phase spatial light modulator LCOS-SLM

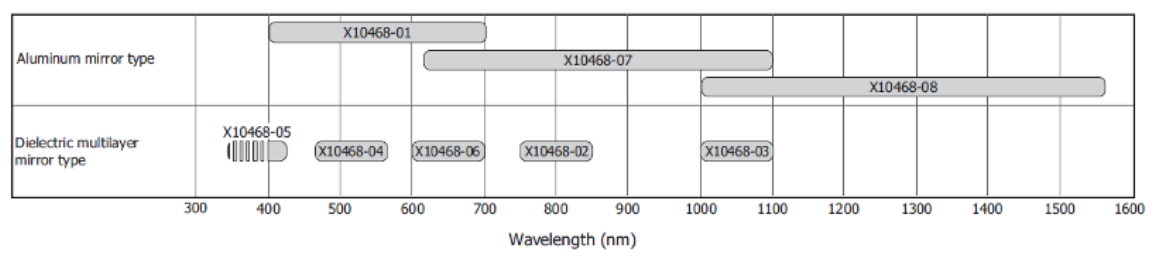

Fig. V.22 - Versions of the X10468 series from Hamamatsu. Image obtained from [56].

The fill factor is another parameter that influences the efficiency of the method. Represents the capacity of the SLM to modulate all light reflected. For instance, the part of the light that passes through the gaps between the pixels of the SLM is not affected by it and cannot be controlled by the technique, becoming non-desired light. The fill factor is very high (93\%) for our device. Finally, the diffraction efficiency is between $65 \%$ and $95 \%$ according to the manufacturer specifications. More information about these parameters can be found in chapter 2. To sum up, putting all these numbers together, we get a final light efficiency of about $50 \%$ just for using the SLM. 
To obtain the real efficiency of our method, we have measured the average power of the beam before the SLM and in the sample plane. The first result, with the patterns employed in left parts of Fig. V.15 and Fig. V.16, reports a $32 \%$ efficiency of our method in comparison with a $23 \%$ obtained with the $\mathrm{CGH}$. However, our method is size-dependent: a bigger pattern leads to a greater efficiency of our method. This is caused because the SLM plane is conjugated with the sample plane, so the bigger the pattern at the sample plane, the more available light at the sample plane. For that reason, a second measurement was performed but only using a very reduced region of the SLM (less than $10 \%$ of the total pixels). In this case, the hologram remains with the same efficiency $23 \%$, but our method has dropped until $18 \%$. Nevertheless, this value is quite similar even in an almost extreme situation, as employing less than $10 \%$ of the available pixels represents an unnecessary loss of spatial resolution.

In the next step, we try to determine the contribution of the diffracted and non-diffracted light to the final images. There is an uncertain amount of light not affected by the SLM phase modulation. In some cases, the non-diffracted light is extremely helpful and enables some possibilities as we have seen with the phase shifting measuring technique [118]. However, generally the effect it produces is detrimental to the experiments, since it only reduces the quality of the images. There are several ways to deal with this zero order. The first one is to try to reduce it as much as possible. As it was previously discussed, part of this light comes from a wrong polarization direction of the beam impinging in the SLM. This contribution can be greatly reduced by simply adding a halfwave plate to set the polarization direction of the beam parallel to the SLM director orientation. Unfortunately, part of the zero order comes from the contribution of non-avoidable sources, i.e. the fill factor. In that regard, one possibility is to design an off-axis optical system, encode a blazing grating at the SLM and filter all but the first diffraction order at the Fourier plane. This solution removes the zero order reducing the efficiency of the method (see chapter 3 for more details). Other possibilities rely on an alternative design of the optical setup to minimize the impact of the non-diffracted light. In our setup, we have opted for this latter possibility. Specifically, we have designed the optical system to force the beam enter the microscope objective $\left(\mathrm{MO}_{1}\right)$ under low-focusing conditions. The common arrangement, where the beam enters the objective under plane-wave configuration, causes the objective to focus non-diffracted light on one intense spot. In our setup, non-diffracted light exits the microscope parallel to the optical axis and not focusing. In this 
way, this light is distributed among the surface of the sample. This results in a quasi-uniform increment of light in all pixels of the image. In the next step, we tried to determine the contribution of the diffracted and non-diffracted light in our patterns. The procedure was to measure the average energy with and without zero order (by using the aforementioned off-axis design). In principle, the difference among both value should correspond to the contribution of the zero order. However, as the off-axis measurement entails a loss of energy (because the encoded blazed grating is not ideal), the contribution of the zero order is overestimated in these results. This calculus indicates that the contribution of the diffracted light is $41 \%$ for the reported technique and $44 \%$ for the CGH measurement. 



\section{Chapter VI - Conclusions}

Along this thesis, a complete overview about encoding techniques employed to spatially manipulate the complex wavefront of femtosecond pulses has been presented. In particular, it is focused on those techniques developed for phase-only SLMs. These devices have been demonstrated as a very powerful tool for a wide range of applications. They are able to control the amplitude or phase of a light wave, but not both simultaneously. Multiple techniques have been reported to take advantage of these devices to achieve complete control over the complex field of laser beams. In particular, a brief discussion about the different technologies to build SLMs has been carried out. Some of the most common technical problems that one can find when working with phaseonly SLM have been investigated. Additionally, why and how to calibrate an SLM is reported. Furthermore, a list of techniques employed to generate arbitrary amplitude patterns is included. For some applications, it is essential to control the entire complex field, not just the amplitude. For this reason, several encoding techniques that allow manipulating the amplitude and phase of laser beams are also included. Finally, one can find in this thesis a list of some relevant applications of beam shaping with SLMs.

\section{VI.1 - Summary of contributions}

The main contributions of this thesis can be divided into three main branches. First, a phase-only SLM is calibrated using a simple but novel technique. The proposed calibration method is based on the encoding of a set of binary Fresnel lenses onto the SLM. A detector is placed at the focal length of these lenses to measure the irradiance of each focus. This calibration was used to optimize the phase response of the device in further experiments. Main advantages of this technique are the lack of an interferometric optical setup, thus avoiding the typical experimental problems associated to these kind of arrangements (sensitivity to environmental fluctuations, need to an extremely precise alignment...) and the reduction of optical components employed in comparison with other calibration methods. In our method, the optical system is quite simple and fast to align. Lastly, as data is measured near focal points, the harmful effect of the zero-order is greatly reduced due to a major contribution of diffracted light.

In a second group, some encoding techniques were developed to arbitrarily control the wave beam. First, I show a technique to generate a three dimensional distribution of foci within a fused silica sample able to carry out 
filamentation inside the crystal. The foci are produced by a DPE made up of a set of spatially multiplexed lenses. The possibility of independently controlling each of these lenses allows us to precisely managing the position of each focus. The main benefit relies in the small separation between filaments that one can achieve with this method. Such separations permit the study of the mutual coherence among filaments due to the interference among each other. In this group, based on the double phase hologram theory, an approach to generate arbitrary complex fields on a certain plane is demonstrated. It employs binary amplitude and complementary masks to perform a spatial sample of the two pre-calculated phase elements. This sampling process allows the encoding of these phase elements onto the SLM. With respect to previous implementations of this method, its application to spatially modify ultrashort pulses is demonstrated for the first time in this thesis. This method presents some advantages derived from the double-phase theory. Among them, the most relevant is that instead of other techniques like IFTAs, we fully encode the complex field. This leads to an effective reduction of speckle noise and spatial irregularities on the illumination pattern. Moreover, the phase control enables some interesting additional features of the system. Apart from the correction of possible aberrations introduced by the optical system, it can be an important tool in nonlinear experiments.

Finally, the proposed techniques are used to achieve promising results in experimental applications. In particular, it is included a contribution where we generate an arbitrary micrometric complex field by using one of the reported methods. This complex field is very convenient for being used within the illumination path of microscopes due to the full control achieved on complex field. In this context, some results that includes second-harmonic generation and two-photon excitation are shown. Additionally, another report presented generates a three dimensional array of foci using this technique. In this case, the phase element encoded into the SLM is calculated using the double phase hologram theory, achieving a very precise control of the focal distributions at the desired planes. Here, experimental results demonstrate that the arbitrary pattern can be used to generate second-harmonic effects on selected regions. By illuminating two spatially separated regions of interest within a sample, it is possible to measure the interference pattern of both regions after light propagation to an arbitrary defocusing plane. This interference pattern is phase-dependent and can be controlled by means of the proposed technique. 


\section{VI.2 - Future work}

Despite all the advances presented in this manuscript, the topic is still a long way ahead. During the development of this thesis, some interesting ideas have emerged and can be realized in the near future. In this section, potential future challenges and improvements in this field are presented to help, as far as possible, future research. One of those ideas lies in the combination of the SLM with a DMD. When performing non-linear experiments, the quality of an image resulting from illuminating a large region of a sample can be seriously affected by the scattering introduced by the sample itself. Scanning systems are less sensitive to this effect because the illuminated region is smaller and it is easier to determine the source of the light; however, the temporal resolution of scanning systems is poor in comparison with our proposal. Since phase-only SLMs have a frame rate of $60 \mathrm{~Hz}$, these devices are not the most appropriate for this task. A faster device would enable the real time acquisition of those processes; however, in the meanwhile we should develop new strategies to achieve faster measurements. Since some processes can only be measured in real time (e.g. synaptic transmissions), improving scan speed is a topic of interest. The addition of a DMD to our system would allow the development of new innovative techniques combining the complex spatial shaping performed by the phase-only SLM with the fast frequency refresh times (dozens of $\mathrm{kHz}$ ) of DMDs. In that sense, for example, the combination of high accuracy multifocal distributions generated by our encoding method with the micrometric spatial modulation due to DMDs can be highly appropriate. For instance, it would allow to reduce the speckle in the recorded images as well as processing information in real time. Once a DMD is included in the system, the element that limits the acquisition speed is the camera. In single-pixel systems, it is common to combine DMDs with point detectors, much faster than conventional cameras [155].

Another idea arisen during this thesis is the application of some of the reported techniques over real biological samples. The developed strategies have been tested here with non-linear crystals but, in principle, after proper preparation similar results should be obtained over biological specimens. In particular, nonlinear microscopy still faces multiple challenges that limit quality of retrieved images e.g., increase the depth of penetration in biological tissue, reduce the scattering, avoid the white noise and improve optical sectioning etc. In addition, targeted illumination allows not only to image samples, but also to trigger trendy strategies like photoactivation of neural circuits [156]. 
Finally, some promising results were obtained in micro-processing of materials [129]. The application of complex field shaping techniques to this topic can enhance it significantly by speeding up the processing of materials. The first steps taken in this regard had develop several ideas to obtain further results. Specifically, the work done proposes a single-shot processing system that allows rapid surface micro-processing of materials using a technique based on the double-phase theory. One of these ideas proposes a different approach. Instead of generating a wide-field pattern, the objective is to generate a threedimensional array of foci to perform a volumetric processing of a material. That way, instead of processing the surface of the object, its interior could also be processed. The same procedure could be employed to generate nanoparticles with laser ablation. In this case, the foci distribution could be generated on the surface of the desired material to achieve the ablation of the material in the form of nano-sized particles. 


\section{Appendix A: Challenges and recent hints of phase- only SLMs}

Predictions about the future of the SLMs are optimistic: the market is growing [157] driven by industrial and scientific topics and, therefore, it is expected that companies will continue to improve the devices they launch for sale. Manufacturers have to face some challenges to improve their products. For instance, laser induced damage threshold is a crucial parameter for applications with high-energy requirements. Recently, some water cooled devices have appeared [158], leading to a dramatic increase of the energy safely handled by the surface of the SLM: from $2 \mathrm{~W} / \mathrm{cm}^{2}$ in the past up to more than $50 \mathrm{~W} / \mathrm{cm}^{2}$ [159]. In any case, these values are achieved only in watercooled devices, with the added complexity of introducing the cooling system within the setup. While it is true that for some applications such as material processing this is enormously relevant, there is still work ahead to increase the damage threshold for all devices. Another parameter that can bring relevant improvements is the resolution, as the more resolution and the smaller the pixel size, the better the approximation of the encoded phase to the theoretical one. Actually there are available on the market $4 \mathrm{~K}$ devices with a pixel pitch smaller than $4 \mu \mathrm{m}$ and a very high fill factor (about 90\%) [160]. Increasing the resolution has some tricky limitations: first, obviously, it entails a considerable increase in the production cost, but also the reduction of the pixel size is limited by the influence of contiguous electric fields. Speed is probably the weakest spot of LCOS SLMs. Recent MEMS-based SLMs are able to achieve $2 \pi$ phase modulation at $10 \mathrm{kHz}$, almost 100 times faster than LCbased SLMs [161]. However, this device lacks the resolution the LC-SLMs have. Increasing the speed of LCoS SLMs is going to be a tough task. The video interface employed in the vast majority of SLMs is either digital visual interface (DVI) or high-definition multimedia interface (HDMI). The main difference between the two is that while the former limits the maximum resolution to $1920 * 1200$, the latter can even distribute a $4 \mathrm{~K}(4096 * 2160)$ signal on the $\mathrm{HDMI} 2.0$ version. These interfaces ensure a frame rate of $60 \mathrm{~Hz}$ for the higher available resolution and the technology seems to continue to improve without problems: The HDMI 2.1 version guarantees $120 \mathrm{~Hz}$ for $4 \mathrm{~K}$ signals [162]. As 4K images are almost four times bigger than the maximum resolution available for the DVI interface. That means that an HDMI cable is capable of sending those images almost four times faster. Finally, other options such as preloading 
the images on the device to show them sequentially later could be explored. That is the good news. The bad news: This is not the only bottleneck. In fact, it might not even be the most restrictive. The response time of the modulator, this is, the time it takes for the LC panel to reach the desired modulation could be the real problem. In Fig. A.1, one can see the response time of the Hamamatsu X10468 series (obtained from [56]).

\begin{tabular}{|c|c|c|c|c|c|c|c|c|}
\hline \multirow{2}{*}{ Type no. } & \multicolumn{8}{|c|}{$\mathrm{X} 10468$} \\
\hline & -01 & -02 & -03 & -04 & -05 & -06 & -07 & -08 \\
\hline Light source wavelength $(\mathrm{nm})$ & 633 & 785 & 1064 & 532 & 407 & 633 & 1064 & 1064 \\
\hline Rise time (ms) & 5 & 30 & 18 & 10 & 6 & 9 & 9 & 11 \\
\hline Fall time (ms) & 26 & 81 & 76 & 23 & 21 & 26 & 81 & 92 \\
\hline
\end{tabular}

Fig. A.1 - Response time of the Hamamatsu X10468 series SLMs. Obtained from [158].

As discussed above, the response is defined as the time needed to switch from $10 \%$ to $90 \%$ of a $2 \pi$ phase shift (rise time) or from $90 \%$ to $10 \%$ (fall time), and it is usually determined by the properties of the LC material, the voltage applied to the cells, temperature, and thickness of the LC layer. There are several conclusions that can be drawn from the figure. First, the rise time and the fall time are completely different, being greater the fall time in all cases. Secondly, some values are higher than desired. Assuming the best case, a rise time of $5 \mathrm{~ms}$ for the first column ( $\mathrm{v}-01$ ). Even in that case, the modulator could encode a maximum of 200 patterns every second, which is clearly inferior to the speed reached by its binary competitors. But not all is lost. Recent studies have reported new panels with sub-millisecond response times [163], and in the market one can already find devices that use alternative backplanes and try to fit the optimal parameters to achieve a reduction on the response time [164]. In addition to the mentioned relevant parameters (LC material, thickness, temperature, and voltage), there seems to be a strong dependence on the response time with the wavelength (see Fig. A.1 and [163]. This can hinder the task of manufacturing faster modulators for some applications that need certain wavelengths. A different approach is explored in [165]. It exploits the transient nematic effect, where the response of the molecules of the $L C$ in a phase change from $\varphi_{0}$ to $\varphi_{1}$ is similar to a logarithmic curve (growths quickly at first, and then it relaxes). To benefit from it, a two-step phase change from $\varphi_{0}$ to $\varphi_{2}$ to $\varphi_{1}$ (where $\varphi_{2}>\varphi_{1}$ ) can be defined. In that way, it takes advantage of the higher initial growth and saturates at the truly desired phase level $\varphi_{1}$, avoiding the final part where the molecules relax. Another novelty reported in [165] is based on the idea that $\varphi_{1}$ and $\varphi_{1}+2 \pi$ are equivalent, and 
sometimes the phase shift between $\varphi_{0}$ and $\varphi_{1}+2 \pi$ is faster than the transition from $\varphi_{0}$ to $\varphi_{1}$.

To sum up, there is still room for improvement in the characteristics of SLMs. The manufacturers continue to improve their devices periodically and, therefore, it is expected that the technology keep improving. As faster, more efficient, more resilient and higher resolution devices appear, new horizons will be explored: new possibilities will appear on the current topics, but also new fields can be interested in include SLMs within their setups. Therefore, it is important to keep abreast of the new developments that occur in this field. 



\section{Resumen en castellano}

Modificar a voluntad las distintas propiedades de la luz ha permitido el avance y aparición de numerosos campos de la investigación científica. La posibilidad de definir, de forma arbitraria, parámetros como el patrón de irradiancia o la fase de un haz láser ha contribuido a adecuar las fuentes de luz a los requisitos concretos de cada usuario para sus distintas aplicaciones. Como resultado, el desarrollo de nuevas técnicas que permitan realizar este tipo de control se ha convertido en un tema esencial que beneficia a gran cantidad de campos, que se pueden aprovechar de estas nuevas mejoras para incrementar el control sobre la fuente de luz empleada en sus investigaciones y procesos industriales.

Los moduladores espaciales de luz (SLMs por sus siglas en inglés) son dispositivos que permiten cierto control directo de estas propiedades. Generalmente, los SLMs son capaces de modular en tiempo real y de forma programable uno de estos parámetros (amplitud o fase). No obstante, para controlar los dos parámetros al mismo tiempo es necesario el empleo de varios dispositivos, o en su defecto el desarrollo de alguna técnica que permita realizar este control con un solo dispositivo.

\section{Objetivos}

Esta tesis está enfocada a revisar las técnicas más notables que permiten realizar una manipulación completa (amplitud y fase) de un haz láser empleando solamente un SLM capaz de controlar la fase. Además, se pretende introducir nuevos métodos que mejoren de alguna forma las técnicas ya existentes. Por otra parte, se resumen algunas de las aplicaciones más notables de este tipo de técnicas, demostrando así la relevancia de la manipulación de haces láser. Por último, para aumentar el control sobre el proceso de manipulación, se pretende estudiar también el comportamiento de los SLMs con el fin de adecuar, en la medida de lo posible, el comportamiento experimental de estos dispositivos a la codificación teórica. De esa manera, es posible mejorar la eficiencia y la resolución del patrón generado por el modulador. 
En concreto los objetivos de esta tesis son los siguientes:

1. Implementar un método capaz de manipular el campo complejo de un haz a partir de una única máscara de fase codificada en un SLM de sólo fase.

2. Emplear este método de codificación para obtener resultados experimentales en aplicaciones novedosas.

3. Estudiar el comportamiento de los SLMs de sólo fase con el objetivo de comprender su funcionamiento experimental y poder compensar los posibles problemas experimentales que pueden reducir la precisión y la eficiencia del método desarrollado.

4. Estudiar métodos alternativos de codificación, tanto para la manipulación de campos complejos como para la manipulación del patrón de irradiancia, con el objetivo de aumentar el conocimiento general sobre el campo y ayudar a la comparación del método desarrollado con técnicas similares. 


\section{References}

1. European Commission, "Key Enabling Technologies | Internal Market, Industry, Entrepreneurship and SMEs," https://ec.europa.eu/growth/industry/policy/key-enablingtechnologies_en.

2. European Technology Platform Photonics21, "A Photonics Private Public Partnership in Horizon2020," https://www.photonics21.org/download/about-us/photonicsppp/photonics-ppp_proposal.pdf?m=1513605498\&.

3. European Technology Platform Photonics21, "Europe's age of light! How photonics will power growth and innovation," https://www.photonics21.org/download/pppservices/photonics-downloads/Europes-age-of-light-PhotonicsRoadmap-C1.pdf.

4. M. Duocastella and C. B. Arnold, "Bessel and annular beams for materials processing," Laser Photon. Rev. 6(5), 607-621 (2012).

5. R. Lu, W. Sun, Y. Liang, A. Kerlin, J. Bierfeld, J. D. Seelig, D. E. Wilson, B. Scholl, B. Mohar, M. Tanimoto, M. Koyama, D. Fitzpatrick, M. B. Orger, and N. Ji, "Video-rate volumetric functional imaging of the brain at synaptic resolution," Nat. Neurosci. 20(4), 620-628 (2017).

6. M. Mazilu, D. J. Stevenson, F. Gunn-Moore, and K. Dholakia, "Light beats the spread: "non-diffracting" beams," Laser Photon. Rev. 4(4), 529-547 (2009). 
7. C. López-Mariscal, J. C. Gutiérrez-Vega, and S. Chávez-Cerda, "Production of high-order Bessel beams with a Mach-Zehnder interferometer," Appl. Opt. 43(26), 5060 (2004).

8. Z. Jaroszewicz, A. Burvall, and A. T. Friberg, "Axicon - the Most Important Optical Element," Opt. Photonics News 16(4), 34 (2005).

9. J. H. McLeod, "The Axicon: A New Type of Optical Element," J. Opt. Soc. Am. 44(8), 592 (1954).

10. S. Logothetidis, P. Delaporte, D. Karnakis, and I. Zergioti, "Laser processing of flexible organic electronic materials," Handb. Flex. Org. Electron. 285-313 (2015).

11. C. Maurer, A. Jesacher, S. Bernet, and M. Ritsch-Marte, "What spatial light modulators can do for optical microscopy," Laser Photon. Rev. 5(1), 81-101 (2011).

12. W. Osten, A. Faridian, P. Gao, K. Körner, D. Naik, G. Pedrini, A. K. Singh, M. Takeda, and M. Wilke, "Recent advances in digital holography [Invited]," Appl. Opt. 53(27), G44 (2014).

13. Y. Hayasaki, T. Sugimoto, A. Takita, and N. Nishida, "Variable holographic femtosecond laser processing by use of a spatial light modulator," Appl. Phys. Lett. 87(3), 031101 (2005).

14. J. Lutkenhaus, D. George, M. Moazzezi, U. Philipose, and Y. Lin, "Digitally tunable holographic lithography using a spatial light modulator as a programmable phase mask," Opt. Express 21(22), 26227 (2013). 
15. B. Jack, J. Leach, H. Ritsch, S. M. Barnett, M. J. Padgett, and S. Franke-Arnold, "Precise quantum tomography of photon pairs with entangled orbital angular momentum," New J. Phys. 11(10), 103024 (2009).

16. M. Padgett and R. Bowman, "Tweezers with a twist," Nat. Photonics 5(6), 343-348 (2011).

17. C. Rosales-Guzmán, A. Forbes, and Society of Photo-optical Instrumentation Engineers, How to Shape Light with Spatial Light Modulators (n.d.).

18. G. R. W. and S. W. O., "A Practical Algorithm for the Determination of Phase from Image and Diffraction Plane Pictures," Optik (Stuttg). 35(2), 237-246 (1972).

19. G. Yang, B. Dong, B. Gu, J. Zhuang, and O. K. Ersoy, "GerchbergSaxton and Yang-Gu algorithms for phase retrieval in a nonunitary transform system: a comparison," Appl. Opt. 33(2), 209 (1994).

20. J. Glückstad and D. Palima, Generalized Phase Contrast: Applications in Optics and Photonics (Springer, 2009).

21. M. R. Hee, E. A. Swanson, J. A. Izatt, J. M. Jacobson, and J. G. Fujimoto, "Femtosecond transillumination optical coherence tomography," Opt. Lett. 18(12), 950 (1993).

22. O. Mendoza-Yero, G. Mínguez-Vega, J. Lancis, E. Tajahuerce, and V. Climent, "Spectral analysis of femtosecond pulse diffraction through binary diffractive optical elements: theory and experiment," Opt. Express 16(4), 2541 (2008). 
23. A. M. Weiner, "Femtosecond optical pulse shaping and processing," Prog. Quantum Electron. 19(3), 161-237 (1995).

24. N. C. Pégard, A. R. Mardinly, I. A. Oldenburg, S. Sridharan, L. Waller, and H. Adesnik, "Three-dimensional scanless holographic optogenetics with temporal focusing (3D-SHOT)," Nat. Commun. 8(1), 1228 (2017).

25. S. Torres-Peiró, J. González-Ausejo, O. Mendoza-Yero, G. Mínguez-Vega, P. Andrés, and J. Lancis, "Parallel laser micromachining based on diffractive optical elements with dispersion compensated femtosecond pulses," Opt. Express 21(26), 31830 (2013).

26. V. Nikolenko, B. O. Watson, R. Araya, A. Woodruff, D. S. Peterka, and R. Yuste, "SLM microscopy: scanless two-photon imaging and photostimulation using spatial light modulators," Front. Neural Circuits 2, 5 (2008).

27. J. P. Vizcaíno, O. Mendoza-Yero, R. Borrego-Varillas, G. MínguezVega, J. R. Vázquez de Aldana, and J. Láncis, "On-axis non-linear effects with programmable Dammann lenses under femtosecond illumination," Opt. Lett. 38(10), 1621 (2013).

28. O. Mendoza-Yero, G. Mínguez-Vega, and J. Lancis, "Encoding complex fields by using a phase-only optical element," Opt. Lett. 39(7), 1740 (2014).

29. W. Wang, S. A. Soper, and S. A. Soper, Bio-MEMS (CRC Press, 2006). 
30. M. Murata, T. Kondoh, T. Yagi, N. Funatsu, K. Tanaka, H. Tsukuni, K. Ohno, H. Usami, R. Nayve, N. Inoue, S. Seto, and N. Morita, "High-Resolution Piezo Inkjet Printhead Fabricated by Three Dimensional Electrical Connection Method using through Glass VIA," in 2009 IEEE 22nd International Conference on Micro Electro Mechanical Systems (IEEE, 2009), pp. 507-510.

31. J. W. Weigold, T. J. Brosnihan, J. Bergeron, and X. Zhang, "A MEMS Condenser Microphone for Consumer Applications," in 19th IEEE International Conference on Micro Electro Mechanical Systems (IEEE, n.d.), pp. 86-89.

32. V. Kaajakari, Practical MEMS (Small Gear Pub, 2009).

33. H. Schenk, A. Wolter, U. A. Dauderstädt, A. Gehner, and H. K. Lakner, "Micro-opto-electro-mechanical systems technology and its impact on photonic applications," J. Micro/Nanolithography, MEMS, MOEMS 4(4), 041501 (2005).

34. T. G. Bifano, T. G. Bifano, B. M. Levine, M. N. Horenstein, J. A. Perreault, T. G. Bifano, B. M. Levine, and M. N. Horenstein, "Adaptive optic correction using microelectromechanical deformable mirrors," Opt. Eng. 41(3), 561 (2002).

35. Y.-X. Ren, R.-D. Lu, and L. Gong, "Tailoring light with a digital micromirror device," Ann. Phys. 527(7)-(8), 447-470 (2015).

36. V. Lerner, D. Shwa, Y. Drori, and N. Katz, "Shaping LaguerreGaussian laser modes with binary gratings using a digital micromirror device," Opt. Lett. 37(23), 4826 (2012). 
37. L. Gong, Y.-X. Ren, G.-S. Xue, Q.-C. Wang, J.-H. Zhou, M.-C. Zhong, Z.-Q. Wang, and Y.-M. Li, "Generation of nondiffracting Bessel beam using digital micromirror device," Appl. Opt. 52(19), 4566 (2013).

38. M. Gottlieb, C. L. M. Ireland, and J. M. Ley, Electro-Optic and Acousto-Optic Scanning and Deflection (M. Dekker, 1983).

39. G. R. B. E. Römer and P. Bechtold, "Electro-optic and Acoustooptic Laser Beam Scanners," Phys. Procedia 56, 29-39 (2014).

40. W. Akemann, J.-F. Léger, C. Ventalon, B. Mathieu, S. Dieudonné, and L. Bourdieu, "Fast spatial beam shaping by acousto-optic diffraction for 3D non-linear microscopy," Opt. Express 23(22), 28191 (2015).

41. M. Schadt, "Construction of a TN-cell and operation of the twisted nematic field effect," https://commons.wikimedia.org/wiki/File:TN-LCD-schematicMS-208kB.png.

42. Z. Zhang, Z. You, and D. Chu, "Fundamentals of phase-only liquid crystal on silicon (LCOS) devices," Light Sci. Appl. 3(10), e213e213 (2014).

43. P. Birch, R. Young, C. Chatwin, M. Farsari, D. Budgett, and J. Richardson, "Fully complex optical modulation with an analogue ferroelectric liquid crystal spatial light modulator," Opt. Commun. 175(4)-(6), 347-352 (2000).

44. D.-K. Yang and S.-T. Wu, Fundamentals of Liquid Crystal Devices (John Wiley, 2006). 
45. V. Durán Bosch, "Optimización del funcionamiento de un modulador espacial de luz de cristal líquido mediante el modelo retardador-rotor. Aplicaciones en óptica adaptativa.," (2007).

46. G. Lazarev, A. Hermerschmidt, S. Krüger, and S. Osten, "LCOS Spatial Light Modulators: Trends and Applications," in Optical Imaging and Metrology (Wiley-VCH Verlag GmbH \& Co. KGaA, 2012), pp. 1-29.

47. B. Apter, U. Efron, and E. Bahat-Treidel, "On the fringing-field effect in liquid-crystal beam-steering devices," Appl. Opt. 43(1), 11 (2004).

48. J. E. Stockley, D. Subacius, and S. A. Serati, "\&lt;title\&gt; Influence of the interpixel region in liquid crystal diffraction gratings\&lt;/title\&gt;", in R. Shashidhar, ed. (International Society for Optics and Photonics, 1999), 3635, pp. 127-136.

49. X. Wang, B. Wang, P. J. Bos, P. F. McManamon, J. J. Pouch, F. A. Miranda, and J. E. Anderson, "Modeling and design of an optimized liquid-crystal optical phased array," J. Appl. Phys. 98(7), 073101 (2005).

50. M. Persson, D. Engström, and M. Goksör, "Reducing the effect of pixel crosstalk in phase only spatial light modulators," Opt. Express 20(20), 22334 (2012).

51. M. Carbonell-Leal and O. Mendoza-Yero, "Effects of mitigation of pixel cross-talk in the encoding of complex fields using the double-phase method," Opt. Eng. 59(04), 1 (2019). 
52. J. García-Márquez, V. López, A. González-Vega, and E. Noé, "Flicker minimization in an LCoS spatial light modulator," Opt. Express 20(8), 8431 (2012).

53. A. Lizana, I. Moreno, A. Márquez, C. lemmi, E. Fernández, J. Campos, and M. J. Yzuel, "Time fluctuations of the phase modulation in a liquid crystal on silicon display: characterization and effects in diffractive optics," Opt. Express 16(21), 16711 (2008).

54. W. P. Bleha and L. A. Lei, "Advances in Liquid Crystal on Silicon (LCOS) spatial light modulator technology," in D. D. Desjardins and K. R. Sarma, eds. (2013), p. 87360A.

55. HOLOEYE Photonics AG, "PLUTO-2 Phase Only Spatial Light Modulator (Reflective)," https://holoeye.com/spatial-lightmodulators/sIm-pluto-phase-only/.

56. Hamamatsu Photonics, "Phase spatial light modulator LCOS-SLM I Chapter 12," https://www.hamamatsu.com/resources/pdf/ssd/e12_handbo ok_lcos_slm.pdf.

57. X. Xun and R. W. Cohn, "Phase calibration of spatially nonuniform spatial light modulators," Appl. Opt. 43(35), 6400 (2004).

58. S. Reichelt, "Spatially resolved phase-response calibration of liquid-crystal-based spatial light modulators," Appl. Opt. 52(12), 2610 (2013). 
59. H. Zhang, A. Lizana, C. lemmi, F. A. Monroy-Ramírez, A. Márquez, I. Moreno, and J. Campos, "LCoS display phase self-calibration method based on diffractive lens schemes," Opt. Lasers Eng. 106, 147-154 (2018).

60. L. Martínez-León, Z. Jaroszewicz, A. Kołodziejczyk, V. Durán, E. Tajahuerce, and J. Lancis, "Phase calibration of spatial light modulators by means of Fresnel images," J. Opt. A Pure Appl. Opt. 11(12), 125405 (2009).

61. I. Moreno, P. Velásquez, C. R. Fernández-Pousa, M. M. SánchezLópez, and F. Mateos, "Jones matrix method for predicting and optimizing the optical modulation properties of a liquid-crystal display," J. Appl. Phys. 94(6), 3697-3702 (2003).

62. D. Engström, M. Persson, J. Bengtsson, and M. Goksör, "Calibration of spatial light modulators suffering from spatially varying phase response," Opt. Express 21(13), 16086 (2013).

63. Z. Zhang, G. Lu, and F. T. S. Yu, "Simple method for measuring phase modulation in liquid crystal televisions," Opt. Eng. 33(9), 3018 (1994).

64. O. Mendoza-Yero, G. Minguez-Vega, L. Martinez-Leon, M. Carbonell-Leal, M. Fernandez-Alonso, C. Donate-Buendia, J. Perez-Vizcaino, and J. Lancis, "Diffraction-Based Phase Calibration of Spatial Light Modulators With Binary Phase Fresnel Lenses," J. Disp. Technol. 12(10), 1027-1032 (2016).

65. E. Buckley, A. Cable, N. Lawrence, and T. Wilkinson, "Viewing angle enhancement for two- and three-dimensional holographic displays with random superresolution phase masks," Appl. Opt. 45(28), 7334 (2006). 
66. P. W. M. Tsang, Y.-T. Chow, and T.-C. Poon, "Generation of phase-only Fresnel hologram based on down-sampling," Opt. Express 22(21), 25208 (2014).

67. M. A. Seldowitz, J. P. Allebach, and D. W. Sweeney, "Synthesis of digital holograms by direct binary search," Appl. Opt. 26(14), 2788 (1987).

68. F. M. Dickey, Laser Beam Shaping: Theory and Techniques (CRC, 2014).

69. R. Di Leonardo, F. Ianni, and G. Ruocco, "Computer generation of optimal holograms for optical trap arrays," Opt. Express 15(4), 1913 (2007).

70. T. Aharoni and S. Shoham, "Phase-controlled, speckle-free holographic projection with applications in precision optogenetics," Neurophotonics 5(02), 1 (2018).

71. R. Conti, O. Assayag, V. de Sars, M. Guillon, and V. Emiliani, "Computer Generated Holography with Intensity-Graded Patterns.," Front. Cell. Neurosci. 10, 236 (2016).

72. A. Jesacher, S. Bernet, and M. Ritsch-Marte, "Colour hologram projection with an SLM by exploiting its full phase modulation range," Opt. Express 22(17), 20530 (2014).

73. F. Zernike, "How I Discovered Phase Contrast," Science (80-. ). 121(3141), 345-349 (1955).

74. J. Glückstad and P. C. Mogensen, "Optimal phase contrast in common-path interferometry," Appl. Opt. 40(2), 268 (2001). 
75. J. Glückstad, D. Palima, P. J. Rodrigo, and C. A. Alonzo, "Laser projection using generalized phase contrast," Opt. Lett. 32(22), 3281 (2007).

76. R. L. Eriksen, P. C. Mogensen, and J. Glückstad, "Multiple-beam optical tweezers generated by the generalized phase-contrast method," Opt. Lett. 27(4), 267 (2002).

77. D. Palima and J. Glückstad, "Diffractive generalized phase contrast for adaptive phase imaging and optical security," Opt. Express 20(2), 1370 (2012).

78. J. A. Davis, D. M. Cottrell, J. Campos, M. J. Yzuel, and I. Moreno, "Encoding amplitude information onto phase-only filters," Appl. Opt. 38(23), 5004 (1999).

79. R. Bowman, N. Muller, X. Zambrana-Puyalto, O. Jedrkiewicz, P. Di Trapani, and M. J. Padgett, "Efficient generation of Bessel beam arrays by means of an SLM," Eur. Phys. J. Spec. Top. 199(1), 159-166 (2011).

80. S. Hasegawa, Y. Hayasaki, and N. Nishida, "Holographic femtosecond laser processing with multiplexed phase Fresnel lenses," Opt. Lett. 31(11), 1705 (2006).

81. R. Borrego-Varillas, J. Perez-Vizcaino, O. Mendoza-Yero, G. Minguez-Vega, J. R. V. de Aldana, and J. Lancis, "Controlled Multibeam Supercontinuum Generation With a Spatial Light Modulator," IEEE Photonics Technol. Lett. 26(16), 1661-1664 (2014). 
82. S. P. Poland, N. Krstajić, S. Coelho, D. Tyndall, R. J. Walker, V. Devauges, P. E. Morton, N. S. Nicholas, J. Richardson, D. D.-U. Li, K. Suhling, C. M. Wells, M. Parsons, R. K. Henderson, and S. M. Ameer-Beg, "Time-resolved multifocal multiphoton microscope for high speed FRET imaging in vivo," Opt. Lett. 39(20), 6013 (2014).

83. R. Borrego-Varillas, C. Romero, O. Mendoza-Yero, G. MínguezVega, I. Gallardo, and J. R. Vázquez de Aldana, "Femtosecond filamentation in sapphire with diffractive lenses," J. Opt. Soc. Am. B 30(8), 2059 (2013).

84. H. Lin and M. Gu, "Creation of diffraction-limited non-Airy multifocal arrays using a spatially shifted vortex beam," Appl. Phys. Lett. 102(8), 084103 (2013).

85. B. Sun, P. S. Salter, C. Roider, A. Jesacher, J. Strauss, J. Heberle, M. Schmidt, and M. J. Booth, "Four-dimensional light shaping: manipulating ultrafast spatiotemporal foci in space and time," Light Sci. Appl. 7(1), 17117 (2018).

86. O. Mendoza-Yero, M. Carbonell-Leal, C. Doñate-Buendía, G. Mínguez-Vega, and J. Lancis, "Diffractive control of 3D multifilamentation in fused silica with micrometric resolution," Opt. Express 24(14), 15307 (2016).

87. G. Mínguez-Vega, J. Lancis, J. Pérez-Vizcaíno, J. R. V. de Aldana, O. Mendoza-Yero, and R. Borrego-Varillas, "Dynamic Control of Interference Effects BetweenOptical Filaments through Programmable Optical PhaseModulation," J. Disp. Technol. Vol. 12, Issue 6, pp. 589-593 12(6), 589-593 (2016). 
88. A. Camino, Z. Hao, X. Liu, and J. Lin, "Control of laser filamentation in fused silica by a periodic microlens array," Opt. Express 21(7), 7908 (2013).

89. J. A. Davis and D. M. Cottrell, "Random mask encoding of multiplexed phase-only and binary phase-only filters," Opt. Lett. 19(7), 496 (1994).

90. C. Iemmi, J. Campos, J. C. Escalera, O. López-Coronado, R. Gimeno, and M. J. Yzuel, "Depth of focus increase by multiplexing programmable diffractive lenses," Opt. Express 14(22), 10207 (2006).

91. C. Maurer, S. Khan, S. Fassl, S. Bernet, and M. Ritsch-Marte, "Depth of field multiplexing in microscopy," Opt. Express 18(3), 3023 (2010).

92. L. G. Neto, D. Roberge, and Y. Sheng, "Full-range, continuous, complex modulation by the use of two coupled-mode liquidcrystal televisions," Appl. Opt. 35(23), 4567 (1996).

93. R. Tudela, E. Martin-Badosa, I. J. I. Labastida, E. Pleguezuelos, S. Vallmitjana, I. P. Juvells, and A. Carnicer, "Full complex Fresnel holograms displayed on liquid crystal devices," in G. C. Righini and A. Consortini, eds. (International Society for Optics and Photonics, 2003), 4829, p. 458.

94. A. Kolodziejczyk, A. Siemion, A. M. Siemion, D. Wojnowski, I. Ducin, J. Suszek, K. Kakarenko, M. Makowski, M. Sypek, and Z. Jaroszewicz, "Complex light modulation for lensless image projection," Chinese Opt. Lett. Vol. 9, Issue 12, pp. 1200089(12), 120008 (2011). 
95. A. Jesacher, C. Maurer, A. Schwaighofer, S. Bernet, and M. Ritsch-Marte, "Near-perfect hologram reconstruction with a spatial light modulator," Opt. Express 16(4), 2597 (2008).

96. L. Zhu and J. Wang, "Arbitrary manipulation of spatial amplitude and phase using phase-only spatial light modulators," Sci. Rep. 4(1), 7441 (2015).

97. V. Parthiban, R. N. Kohn, J. Liang, and M. F. Becker, "Experimental demonstration of precise holograms using complex light modulation," in M. R. Douglass, P. S. King, and B. L. Lee, eds. (International Society for Optics and Photonics, 2016), 9761, p. 97610M.

98. S. A. Goorden, J. Bertolotti, and A. P. Mosk, "Superpixel-based spatial amplitude and phase modulation using a digital micromirror device," Opt. Express 22(15), 17999 (2014).

99. D. Bowman, T. L. Harte, V. Chardonnet, C. De Groot, S. J. Denny, G. Le Goc, M. Anderson, P. Ireland, D. Cassettari, and G. D. Bruce, "High-fidelity phase and amplitude control of phase-only computer generated holograms using conjugate gradient minimisation," Opt. Express 25(10), 11692 (2017).

100. A. Shibukawa, A. Okamoto, M. Takabayashi, and A. Tomita, "Spatial cross modulation method using a random diffuser and phase-only spatial light modulator for constructing arbitrary complex fields," Opt. Express 22(4), 3968 (2014).

101. E. Bolduc, N. Bent, E. Santamato, E. Karimi, and R. W. Boyd, "Exact solution to simultaneous intensity and phase encryption with a single phase-only hologram," Opt. Lett. 38(18), 3546 (2013). 
102. J. Luis Martínez Fuentes and I. Moreno, "Random technique to encode complex valued holograms with on axis reconstruction onto phase-only displays," Opt. Express 26(5), 5875 (2018).

103. N. Suchkov, E. J. Fernández, J. L. Martínez-Fuentes, I. Moreno, and P. Artal, "Simultaneous aberration and aperture control using a single spatial light modulator," Opt. Express 27(9), 12399 (2019).

104. C. K. Hsueh and A. A. Sawchuk, "Computer-generated doublephase holograms," Appl. Opt. 17(24), 3874 (1978).

105. H. Song, G. Sung, S. Choi, K. Won, H.-S. Lee, and H. Kim, "Optimal synthesis of double-phase computer generated holograms using a phase-only spatial light modulator with grating filter," Opt. Express 20(28), 29844 (2012).

106. J.-P. Liu, W.-Y. Hsieh, T.-C. Poon, and P. Tsang, "Complex Fresnel hologram display using a single SLM," Appl. Opt. 50(34), H128 (2011).

107. S. Reichelt, R. Häussler, G. Fütterer, N. Leister, H. Kato, N. Usukura, and Y. Kanbayashi, "Full-range, complex spatial light modulator for real-time holography," Opt. Lett. 37(11), 1955 (2012).

108. V. Bagnoud and J. D. Zuegel, "Independent phase and amplitude control of a laser beam by use of a single-phase-only spatial light modulator," Opt. Lett. 29(3), 295 (2004).

109. E. G. van Putten, I. M. Vellekoop, and A. P. Mosk, "Spatial amplitude and phase modulation using commercial twisted nematic LCDs," Appl. Opt. 47(12), 2076 (2008). 
110. V. Arrizón, "Complex modulation with a twisted-nematic liquidcrystal spatial light modulator: double-pixel approach," Opt. Lett. 28(15), 1359 (2003).

111. Y. Qi, C. Chang, and J. Xia, "Speckleless holographic display by complex modulation based on double-phase method," Opt. Express 24(26), 30368 (2016).

112. L. Gong, W. Liu, Q. Zhao, Y. Ren, X. Qiu, M. Zhong, and Y. Li, "Controllable light capsules employing modified Bessel-Gauss beams," Sci. Rep. 6(1), 29001 (2016).

113. J. Hu, C.-S. Brès, and C.-B. Huang, "Talbot effect on orbital angular momentum beams: azimuthal intensity repetition-rate multiplication," Opt. Lett. 43(16), 4033 (2018).

114. C. Chang, Y. Gao, J. Xia, S. Nie, and J. Ding, "Shaping of optical vector beams in three dimensions," Opt. Lett. 42(19), 3884 (2017).

115. Y. Zha, K. Huang, B. Liu, M. Sun, H. Hu, N. Li, X. Zhang, B. Zhu, and X. Lu, "Elliptical Airy beam," Appl. Opt. 57(23), 6717 (2018).

116. T. Li, F. Zi, K. Huang, and X. Lu, "Multifocus autofocusing Airy beam," J. Opt. Soc. Am. A 34(9), 1530 (2017).

117. M. Carbonell-Leal and O. Mendoza-Yero, "Shaping the Amplitude and Phase of Laser Beams by Using a Phase-only Spatial Light Modulator," J. Vis. Exp. (143), e59158 (2019). 
118. I. Yamaguchi and T. Zhang, "Phase-shifting digital holography," Opt. Lett. 22(16), 1268 (1997).

119. S. Sinzinger and J. Jahns, Microoptics (Wiley, 2003).

120. F. Vargas-Martín, P. M. Prieto, and P. Artal, "Correction of the aberrations in the human eye with a liquid-crystal spatial light modulator: limits to performance," J. Opt. Soc. Am. A 15(9), 2552 (1998).

121. P. S. Salter, M. Baum, I. Alexeev, M. Schmidt, and M. J. Booth, "Exploring the depth range for three-dimensional laser machining with aberration correction," Opt. Express 22(15), 17644 (2014).

122. A. M. Weiner, "Femtosecond pulse shaping using spatial light modulators," Rev. Sci. Instrum. 71(5), 1929-1960 (2000).

123. J.-H. Park, "Recent progress in computer-generated holography for three-dimensional scenes," J. Inf. Disp. 18(1), 1-12 (2017).

124. A. Jesacher, C. Maurer, A. Schwaighofer, S. Bernet, and M. Ritsch-Marte, "Full phase and amplitude control of holographic optical tweezers with high efficiency," Opt. Express 16(7), 4479 (2008).

125. K. Dholakia and T. Čižmár, "Shaping the future of manipulation," Nat. Photonics 5(6), 335-342 (2011).

126. M. A. Karim and A. A. S. Awwal, "Electrooptic displays for optical information processing," Proc. IEEE 84(6), 814-827 (1996). 
127. I. Moreno, J. A. Davis, T. M. Hernandez, D. M. Cottrell, and D. Sand, "Complete polarization control of light from a liquid crystal spatial light modulator," Opt. Express 20(1), 364 (2012).

128. Z. Kuang, W. Perrie, J. Leach, M. Sharp, S. P. Edwardson, M. Padgett, G. Dearden, and K. G. Watkins, "High throughput diffractive multi-beam femtosecond laser processing using a spatial light modulator," Appl. Surf. Sci. 255(5), 2284-2289 (2008).

129. O. Mendoza-Yero, M. Carbonell-Leal, G. Minguez-Vega, C. Donate-Buendia, and J. R. V. de Aldana, "Single-shot femtosecond laser ablation of wide-field irradiance patterns onto a silicon sample," in 2017 Conference on Lasers and ElectroOptics Europe \& European Quantum Electronics Conference (CLEO/Europe-EQEC) (IEEE, 2017), pp. 1-1.

130. T. J. Mclntyre, C. Maurer, S. Fassl, S. Khan, S. Bernet, and M. Ritsch-Marte, "Quantitative SLM-based differential interference contrast imaging," Opt. Express 18(13), 14063 (2010).

131. M. P. Lee, G. M. Gibson, R. Bowman, S. Bernet, M. Ritsch-Marte, D. B. Phillips, and M. J. Padgett, "A multi-modal stereo microscope based on a spatial light modulator," Opt. Express 21(14), 16541 (2013).

132. E. Wolf, "Three-dimensional structure determination of semitransparent objects from holographic data," Opt. Commun. 1(4), 153-156 (1969). 
133. S. Shin, K. Kim, T. Kim, J. Yoon, K. Hong, J. Park, and Y. Park, "Optical diffraction tomography using a digital micromirror device for stable measurements of 4D refractive index tomography of cells," in G. Popescu and Y. Park, eds. (International Society for Optics and Photonics, 2016), 9718, p. 971814.

134. Y. Sung, W. Choi, C. Fang-Yen, K. Badizadegan, R. R. Dasari, and M. S. Feld, "Optical diffraction tomography for high resolution live cell imaging," Opt. Express 17(1), 266 (2009).

135. K. Kim, J. Yoon, S. Shin, S. Lee, S.-A. Yang, and Y. Park, "Optical diffraction tomography techniques for the study of cell pathophysiology," J. Biomed. Photonics Eng. 2(2), 020201-1020201-16 (2016).

136. R. K. Tyson, Principles of Adaptive Optics (n.d.).

137. M. J. Booth, "Adaptive optics in microscopy," Philos. Trans. R. Soc. A Math. Phys. Eng. Sci. 365(1861), 2829-2843 (2007).

138. S. R. P. Pavani, M. A. Thompson, J. S. Biteen, S. J. Lord, N. Liu, R. J. Twieg, R. Piestun, and W. E. Moerner, "Three-dimensional, single-molecule fluorescence imaging beyond the diffraction limit by using a double-helix point spread function.," Proc. Natl. Acad. Sci. U. S. A. 106(9), 2995-9 (2009).

139. I. M. Vellekoop and A. P. Mosk, "Focusing coherent light through opaque strongly scattering media," Opt. Lett. 32(16), 2309 (2007). 
140. S. Popoff, G. Lerosey, M. Fink, A. C. Boccara, and S. Gigan, "Image transmission through an opaque material," Nat. Commun. 1(1), 81 (2010).

141. O. Katz, E. Small, Y. Bromberg, and Y. Silberberg, "Focusing and compression of ultrashort pulses through scattering media," Nat. Photonics 5(6), 372-377 (2011).

142. Y. Guan, O. Katz, E. Small, J. Zhou, and Y. Silberberg, "Polarization control of multiply scattered light through random media by wavefront shaping," Opt. Lett. 37(22), 4663 (2012).

143. V. Ntziachristos, "Going deeper than microscopy: the optical imaging frontier in biology," Nat. Methods 7(8), 603-614 (2010).

144. J. A. Davis, I. Moreno, J. L. Martínez, T. J. Hernandez, and D. M. Cottrell, "Creating three-dimensional lattice patterns using programmable Dammann gratings," Appl. Opt. 50(20), 3653 (2011).

145. G. Sinclair, J. Leach, P. Jordan, G. Gibson, E. Yao, Z. J. Laczik, M. J. Padgett, and J. Courtial, "Interactive application in holographic optical tweezers of a multi-plane Gerchberg-Saxton algorithm for three-dimensional light shaping," Opt. Express 12(8), 1665 (2004).

146. D. Tanese, J.-Y. Weng, V. Zampini, V. De Sars, M. Canepari, B. Rozsa, V. Emiliani, and D. Zecevic, "Imaging membrane potential changes from dendritic spines using computer-generated holography," Neurophotonics 4(3), 031211 (2017). 
147. K.-P. Proll, J.-M. Nivet, K. Körner, and H. J. Tiziani, "Microscopic three-dimensional topometry with ferroelectric liquid-crystalon-silicon displays," Appl. Opt. 42(10), 1773 (2003).

148. A. Li, X. Peng, Y. Yin, X. Liu, Q. Zhao, K. Körner, and W. Osten, "Fringe projection based quantitative 3D microscopy," Optik (Stuttg). 124(21), 5052-5056 (2013).

149. Y. Yin, M. Wang, B. Z. Gao, X. Liu, and X. Peng, "Fringe projection $3 \mathrm{D}$ microscopy with the general imaging model," Opt. Express 23(5), 6846 (2015).

150. O. Mendoza-Yero, M. Carbonell-Leal, G. Mínguez-Vega, and J. Lancis, "Generation of multifocal irradiance patterns by using complex Fresnel holograms," Opt. Lett. 43(5), 1167 (2018).

151. S. Katayama, C. Tateno, T. Asahara, and K. Yoshizato, "Sizedependent in vivo growth potential of adult rat hepatocytes.," Am. J. Pathol. 158(1), 97-105 (2001).

152. P. F. Millington and R. Wilkinson, Skin (Cambridge University Press, 2009).

153. M. Carbonell-Leal, G. Mínguez-Vega, J. Lancis, and O. MendozaYero, "Encoding of arbitrary micrometric complex illumination patterns with reduced speckle," Opt. Express 27(14), 19788 (2019).

154. R. Bäumner, L. Bonacina, J. Enderlein, J. Extermann, T. FrickeBegemann, G. Marowsky, and J.-P. Wolf, "Evanescent-FieldInduced Second Harmonic Generation by Noncentrosymmetric Nanoparticles," Opt. Express 18(22), 23218 (2010). 
155. H. González, L. Martínez-León, F. Soldevila, M. Araiza-Esquivel, J. Lancis, and E. Tajahuerce, "High sampling rate single-pixel digital holography system employing a DMD and phase-encoded patterns," Opt. Express 26(16), 20342 (2018).

156. V. Szabo, C. Ventalon, V. De Sars, J. Bradley, and V. Emiliani, "Spatially Selective Holographic Photoactivation and Functional Fluorescence Imaging in Freely Behaving Mice with a Fiberscope," Neuron 84(6), 1157-1169 (2014).

157. Reuters, "Spatial Light Modulator Market 2019 Size, Share, Trends, Demand, Historical Analysis, Gross Margin, Industry Growth by Regional Forecast to 2023," https://www.reuters.com/brandfeatures/venturecapital/article id=86403.

158. Hamamatsu Photonics, "LCOS-SLM X10468/X13267/X13138 series technical specifications," https://www.hamamatsu.com//resources/pdf/ssd/x10468_seri es_etc_kacc1172e.pdf.

159. G. Lazarev, P.-J. Chen, J. Strauss, N. Fontaine, and A. Forbes, "Beyond the display: phase-only liquid crystal on Silicon devices and their applications in photonics [Invited]," Opt. Express 27(11), 16206 (2019).

160. HOLOEYE Photonics AG, "GAEA-2 10 Megapixel Phase Only LCOS-SLM," https://holoeye.com/gaea-4k-phase-only-spatiallight-modulator/.

161. B. Blochet, L. Bourdieu, and S. Gigan, "Focusing light through dynamical samples using fast continuous wavefront optimization," Opt. Lett. 42(23), 4994 (2017). 
162. I. HDMI Licensing Administrator, "HDMI 2.1 technical specifications,"

https://www.hdmi.org/manufacturer/hdmi_2_1/index.aspx.

163. J.-P. Yang, H.-M. P. Chen, Y. Huang, Y.-C. Chang, F.-W. Lai, S.-T. Wu, C. Hsu, R. Tsai, and R. Hsu, "66-3: Submillisecond-Response 10-Megapixel 4K2K LCoS for Microdisplay and Spatial Light Modulator," SID Symp. Dig. Tech. Pap. 50(1), 933-936 (2019).

164. Meadowlark Optics, "Meadowlark Optics SLM Selection Guide," https://photonlines-recherche.fr/wpcontent/uploads/2018/12/selection_guide_slm.pdf.

165. A. M. Linnenberger, "Advanced SLMs for microscopy," in Adaptive Optics and Wavefront Control for Biological Systems IV, T. G. Bifano, S. Gigan, and J. Kubby, eds. (SPIE, 2018), 10502, p. 3. 
Publications 


\title{
Effects of mitigation of pixel crosstalk in the encoding of complex fields by using double-phase method
}

\author{
Miguel Carbonell-Leal, ${ }^{a}$ and Omel Mendoza-Yero, ${ }^{a}, *$ \\ ${ }^{a}$ GROC•UJI, Institute of New Imaging Technologies, UniversitatJaume I, Castelló, Spain, Postal Code 12071
}

\begin{abstract}
In this contribution we report on the unwanted effects of pixel crosstalk and its mitigation on the experimental realization of double-phase method with phase-only spatial light modulators. We experimentally demonstrate that a generalized sampling scheme can reduce non-uniform phase modulation due to the pixel crosstalk phenomenon, and consequently improve the quality of amplitude and phase images obtained with this encoding method. To corroborate our proposal, a couple of experiments addressed to reconstruct amplitude-only as well as fully independently amplitude and phase patterns under different spatial sampling schemes were carried out. In this context, we also show how a novel implementation of the well-known polarization-based phase shifting technique can be employed to measure the encoded complex field using only a conventional CMOS camera.
\end{abstract}

Keywords: diffractive optics, beam control, phase modulation, interferometry, liquid crystal devices, pixel crosstalk.

*Omel Mendoza-Yero, E-mail: omendoza@fca.uji.es

\section{Introduction}

At present, there are a wide variety of reported optical methods addressed to optically manipulate the complex field of laser beams by using spatial light modulators ${ }^{1-14}$. Among them, those ones based on the use of parallel aligned liquid crystal on silicon (PA-LCoS) SLMs have gain special attention not only because of their relative high efficiency, but also due to their proved ability to accurately modify the physical behavior of laser beams by using just a single phase element encoded into a phase-only $\mathrm{SLM}^{3,4,7,8-14}$. In this context, among many other applications and/or signal displaying methods, computer generated-holograms have been synthetized by means of conventional iterative Fourier transform algorithms ${ }^{15}$, employing a search, also iterative, algorithm able to generate binary holograms ${ }^{16}$, by using a complex amplitude modulation method for 3D dynamic holographic display ${ }^{17}$, by the application of the one step phase retrieval approach which allows a rapid computation of phase-only holograms ${ }^{18}$, by down-sample the intensity image with 
uniform grid-cross lattices ${ }^{19}$, or more recently by adding a periodic phase pattern to the source image $^{20}$. Here, we deal with a particular interferometric method ${ }^{21}$ aimed to encode and retrieve the complex field of coherent laser beams. This method has been widely and successfully employed in several experimental tasks including, but not limited to, demonstrate the Talbot self-imaging in the azimuthal angle ${ }^{22}$, generate speckleless holographic displays ${ }^{23}$, trap magnetic microparticles employing Bessel-Gauss beams $s^{24}$, shape optical vector beams ${ }^{25}$, or experimentally investigate the propagation and focusing characteristics of Airy beams ${ }^{26,27}$. In all these applications the implementation of the above-mentioned encoding method ${ }^{21}$ was carried out with the help of a commercially available PA-LCoS SLM. So, light undergoes phase modulation due to a change in the refractive index of the nematic liquid crystal (LC) material. Specifically, the phase shift is associated with the tilt of the SLM molecules when a signal voltage is applied between the front and the back faces of each LC cell. In addition, main design features of these devices ensure, in principle, that the phase modulation process is done with almost no coupling of amplitude modulation or change in the polarization state of the incident light, which is highly desirable for applications involving interference or diffraction phenomena.

However, PA-LCoS SLMs are not ideal devices but show some effects that cause degradation of the phase modulation response. For instance, temporal fluctuations of the LC molecular orientation as a function of time causes depolarization effects, deteriorating the diffraction efficiency of $\mathrm{SLM}^{28}$. Another harmful effect is related to the Fabry-Perot multiple beam interference generated by the intrinsic layer structure of the LC device ${ }^{29}$ that may originate non-linear phase modulation or even some coupling of amplitude modulation. In this context, there is a particular unwanted effect that becomes critical for applications that require encoded patterns with abrupt phase discontinuities, e.g., diffraction gratings with few phase levels or phase distributions associated 
with high scattering media. In the literature, this widely-studied phenomenon ${ }^{30-33}$ known as fringing field effect (or pixel crosstalk effect) can produce variations in the orientation of LC molecules at adjacent cells, and consequently modify its expected phase response. Hence, as in the double-phase method ${ }^{21}$ the encoded element is generated by the spatial multiplexing of two phase patterns at Nyquist limit, one might expect that its experimental realization with PA-LCoS SLMs does not lack from pixel crosstalk effects.

In this contribution we experimentally show that pixel crosstalk effects do deteriorate the amplitude and phase patterns obtained due to the application of the double-phase method ${ }^{21}$. To alleviate these problems, a generalized sampling scheme able to significantly reduce non-uniform phase modulation without compromising the accuracy of the method is proposed. In order to avoid the influence of the zero order coming from the SLM on the measurements done throughout this work, a fixed blazed grating was added to each phase element sent to the SLM, in the same manner as proposed $\mathrm{in}^{23}$.

\section{Fundamentals of the proposal}

The theory underlying double-phase method ${ }^{21}$ can be briefly described as follow. Any complex field represented in the form $U(x, y)=A(x, y) e^{i \varphi(x, y)}$ can be also rewritten as:

$$
U(x, y)=e^{i \theta(x, y)}+e^{i \vartheta(x, y)},
$$

where,

$$
\begin{aligned}
& \theta(x, y)=\varphi(x, y)+\cos ^{-1}\left[A(x, y) / A_{\text {max }}\right], \\
& \vartheta(x, y)=\varphi(x, y)-\cos ^{-1}\left[A(x, y) / A_{\text {max }}\right],
\end{aligned}
$$

In Eqs. 1-3, the amplitude and phase of the two-dimensional complex field $U(x, y)$ is given by $A(x, y)$ and $\varphi(x, y)$, respectively. In addition, $A_{\max } \equiv 2$ holds for the maximum of $A(x, y)$. From 
Eq. (1), it is apparent that $U(x, y)$ can be obtained from the coherent sum of the uniform waves $e^{i \theta(x, y)}$ and $e^{i \vartheta(x, y)}$. To do that using just a phase-only SLM, above uniform waves are spatially multiplexed with two-dimensional complementary binary gratings (please, see Fig. 1). This allows us to get a single phase element $\alpha(x, y)$ as follows:

$$
M_{1}(x, y) e^{i \theta(x, y)}+M_{2}(x, y) e^{i \vartheta(x, y)}=e^{i \alpha(x, y)},
$$

where,

$$
\alpha(x, y) \equiv M_{1}(x, y) \theta(x, y)+M_{2}(x, y) \vartheta(x, y)
$$

At this point, the interference of above-mentioned uniform waves cannot happen if we do not mix the information contained in the phase element $\alpha(x, y)$. This is carried out by using a spatial filter able to block all diffraction orders but the zero one. Here, it should be mentioned that for the off axis setup employed in this work the filtering process is done around the order at which blazed grating achieves its maximum diffraction efficiency.

It can be shown that, after this filtering process, the spectrum of the original complex field at the Fourier plane can be exactly retrieved. Consequently, at the output plane of the imaging system, the retrieved complex field $U_{R E T}(x, y)$, (without considering constant factors), is given by the convolution of the magnified and spatially reversed complex field $U(x, y)$ with the Fourier transform of the filter mask, that is:

$$
U_{R E T}(x, y)=U(-x / M a g,-y / M a g) \otimes F\{P(u, v)\}
$$

In Eq. (6), the convolution and Fourier transform operations are denoted by the symbols $\otimes$ and $F\{\}$, respectively. Additionally, the term Mag represents the magnification of the imaging system, whereas $P(u, v)$ is the filter function with coordinates $u$ and $v$ in the frequency space. So, from Eq. (6), in theory, the amplitude and phase of the original complex field is fully retrieved, except for some loss of spatial resolution due to the convolution operation. 
In practice, the real physical behavior of phase-only SLM devices under extreme pixel-to-pixel phase modulation conditions can originate discrepancies between the theory and experiment. This mainly happens because the phase information associated with each uniform wave is spatially multiplexed at the Nyquist limit. However, if the period of the binary gratings is not taken at the Nyquist limit, but the spatial frequency separations of diffraction orders are great enough to avoid overlapping among them, the Whittaker-Shannon sampling theorem ensures that, for bandlimited functions, the reconstruction of the spectrum at the Fourier plane is still accomplished exactly. Hence, the utilization of phase elements $\alpha(x, y)$ computer generated from binary gratings with more than 1 pixel per cell should alleviate unwanted crosstalk effects.

\section{Experimental corroboration of the proposal}

In order to show the effects of pixel crosstalk on the retrieved complex field and how mitigatethem we carry out a couple of experiments. The optical setup used for both experiments is shown in Fig. 2. As light source, we use a quasi-monochromatic laser beam of $10 \mathrm{~nm}$ spectral width and centered at $800 \mathrm{~nm}$.Before it impinges onto a reflective phase-only PA-LCoS SLM (Holoeye Pluto optimized for $700 \mathrm{~nm}-1000 \mathrm{~nm}$, resolution $1920 \times 1080$ pixels, pixel pitch $8 \mu \mathrm{m}$, and phase range $3 \pi$ ), the beam is conveniently attenuated with neutral filters (NF), and spatially magnified by using a commercial (BE06R) reflective 6X beam expander (BE). Then, the beam is sent to the SLM forming a small angle with the normal to the LC surface of about 4 degrees, and is back reflected towards the entrance of a $4 \mathrm{f}$ imaging system. The input plane of this optical system coincides with the SLM plane. The $4 \mathrm{f}$ imaging system is made up of a couple of refractive lenses $\left(\mathrm{L}_{1}\right.$ and $\mathrm{L}_{2}$ ) with focal lengths of $1 \mathrm{~m}$ and $0.5 \mathrm{~m}$, respectively. This pair of lenses gives a transversal demagnification of $1 / 2$ at the output plane of the imaging system. This reduction allows us to directly measure the irradiances with a CMOS camera (Ueye UI-1540M, $1280 \times 1024$ pixel resolution and 
5.2 pixel pitch). Non-diffracted light was removed from the setup by using a blazed grating having a fixed period $\mathrm{p}=160 \mu \mathrm{m}$, and encoded from $-\pi$ to $\pi$ with 20 phase levels per period. At the Fourier plane, the beam is transmitted through a low-pass spatial filter that consists of a hard circular iris (ID12Z/M). The iris size was adjusted each time to fulfill the filtering condition.

In the first experiment, an amplitude-only pattern given by a mushroom image is encoded with double-phase method ${ }^{21}$ into the SLM by using three different sampling gratings. This encoding process is exemplified in Fig. 1 with a trivial example. For this experiment we employ binary gratings of 1x1, 4x4, and 10x10 pixels per cell. The measured amplitude images are shown in Fig. 3.

If we compare the original image given in Fig. 3(a) with the remaining ones, it is clear that image quality is affected by the non-uniform phase response of the SLM. At the Nyquist limit (see Fig. 3(b)), the recorded image has the best resolution, but its brightness is so high that contrast becomes really poor. When pixel-cell size is increased up to 16 pixels per cell, the image contrast and sharpness are greatly improved at expense of a little decrease of resolution, please see Fig. 3(c). This can be regarded as an optimal situation, because crosstalk effects are mitigated and image resolution is still acceptable. However, if pixel-cell is increased too much, like in Fig. 3(d) with 100 pixels per cell, problems related to the loss of resolution predominate over any potential improvement in the image quality.

In the second experiment, we use double-phase method ${ }^{21}$ to reconstruct a non-trivial complex field under the sampling configuration of $1 \times 1,2 \times 2$, and $5 \times 5$ pixels per cell. In this case, an original implementation of the polarization-based phase shifting technique ${ }^{34}$ is applied to measure the retrieved complex field. This implementation can be explained by the following three steps. In the first one, after multiplying the phase element $\alpha(x, y)$ by an additional two-dimensional binary 
grating $M_{3}(x, y)$, some pixel-cells of $\alpha(x, y)$ are periodically eliminated. Particularly, $M_{3}(x, y)$ has double the period of $M_{1}(x, y)$ or $M_{2}(x, y)$. In Fig. 4, this first step is shown by means of two examples corresponding to trivial cases given in Fig. 1.

In the second step the blazed grating is added to the modified phase element $\alpha_{M}(x, y)$. Finally, in the last step the interferograms are determined with the help of four uniform phases with values of $0, \pi / 2, \pi$, and $3 \pi / 2$ radians, previously multiplied by the complementary of $M_{3}(x, y)$, that are also added to $\alpha_{M}(x, y)$ before sending it to the SLM. The interferograms are formed after recombining both the light coming from the eliminated pixel-cells (reference beam), and the light modulated by the SLM at the remaining pixel-cells (object beam). The main advance of this procedure is that there is no need of extra optical elements (like polarizers) to obtain the interferograms. However, this is done at expense of a loss of resolution of the retrieved images because now the filtering process is more severe. That is; the filtering condition should be accommodated to the period of $M_{3}(x, y)$ instead of that corresponding to $M_{1}(x, y)$ or $M_{2}(x, y)$.

For this experiment, the amplitude and phase of the complex field are given by images of a young boy and girl, respectively, see Figure 5(a) and (b). Additionally, in Fig. 5(c), (e), and (g) appear the recovered amplitude images when employing binary gratings of $1 \times 1,2 \times 2$, and $5 \times 5$ pixels per cell, whereas the corresponding phase images are given in Fig. 5(d), (f), and (h).

From the experimental results shown in Fig. 5, one can confirm again that pixel crosstalk produces negative effects in the quality of the recorded complex field. In particular, the contrast of amplitude patterns are clearly deteriorated when decreasing the pixel-cell size whereas, phase patterns seem to be poorly changed by the same reason. This last fact can be better undertook if we rewrite the phase element as:

$$
\alpha(x, y)=\varphi(x, y)+\Theta(x, y),
$$


where,

$$
\Theta(x, y)=M_{1} \cos ^{-1}\left[\frac{A(x, y)}{A_{\max }}\right]-M_{2} \cos ^{-1}\left[\frac{A(x, y)}{A_{\max }}\right],
$$

From Eq. (7) and (8) one can realize that, in the double-phase method ${ }^{21}$ the original phase $\varphi(x, y)$ is fully encoded into the SLM. In addition, the employed optical imaging system ensures a replica of $\varphi(x, y)$ at the output plane. So, any loss of resolution of phase images should be mainly caused by the filtering process. However, the term $\Theta(x, y)$ is directly related to the encoding of amplitude information, which can only be retrieved by means of the interference among nearby pixels. So, as the interference process strongly depends on the phase values of these pixels, the amplitude images are definitively more spoiled by the pixel crosstalk effects than phase ones. These conclusions were supported by the calculus of the rmse between the original and measured patterns. It yields rmse of $23.5 \%, 21.6 \%$ and $19.5 \%$ for the amplitude patterns encoded with binary gratings of $1 \times 1,2 \times 2$, and $5 \times 5$ pixels per cells, whereas for the corresponding phase patterns, the numbers were $7.9 \%, 8.2 \%$ and $9.1 \%$.

\section{Conclusions}

In this manuscript we have discussed and partially compensated the effects of pixel crosstalk on double-phase method ${ }^{21}$ implemented with phase-only PA-LCoS SLMs. Our experiments show that non-uniform phase modulation due to pixel crosstalk may deteriorate the quality of reconstructed images which basically reaching less sharpness and contrast than expected. Hence, to mitigate unwanted crosstalk effects we propose and experimentally demonstrate a generalized sampling scheme which preserves the accuracy of the original double-phase method. We found that a slight increase of the grating's period beyond the Nyquist limit significantly reduces crosstalk effects. In addition, light efficiency should be also benefited from the better phase response of the liquid 
crystal display through the availability of more diffracted light. However, this is always happen at expense of decreasing the spatial resolution of the reconstructed hologram in the manner described by Eq. (6). In this contribution, the measurements of the amplitude and phase patterns associated with the complex field were carried out with a novel implementation of the polarization-based phase shifting technique ${ }^{34}$.

We believe that results shown here can be useful to improve next experimental realizations of the double-phase method ${ }^{21}$ with phase-only PA-LCoS SLMs. At this point, it is apparent that degree of damage in the reconstructed images should depend on the employed SLM and its technical specifications. That is why, the selection of an optimum period for the binary gratings can vary from one SLM to another, and it is clearly influenced by the final application.

\subsection{Disclosures}

There are no conflicts of interest to declare.

\subsection{Acknowledgments}

The authors are also very grateful to the SCIC of the Universitat Jaume I for the use of the femtosecond laser.

\subsection{References}

1. V. Arrizón, G. Méndez and D. Sánchez-de-La-Llave, "Accurate encoding of arbitrary complex fields with amplitude-only liquid crystal spatial light modulators," Opt. Express 13(20), $7913-7927$ (2005).

2. E. Ulusoy, L. Onural, and H. M. Ozaktas, "Full-complex amplitude modulation with binary spatial light modulators," J. Opt. Soc. Am. A. 28(11), 2310-2321 (2011). 
3. H. Songet al., "Optimal synthesis of double-phase computer generated holograms using a phase-only spatial light modulator with grating filter," Opt. Express 20(28), 29844-29853 (2012).

4. S. Reichelt et al., "Full-range, complex spatial light modulator for real-time holography," Opt. Lett. 37(11), 1955-1957 (2012).

5. S. A. Goorden, J. Bertolotti, and A. P. Mosk, "Superpixel-based spatial amplitude and phase modulation using a digital micromirror device," Opt. Express 22(15), 17999-18009 (2014) [doi:10.1364/OE.22.017999].

6. L. Zhu and J. Wang, “Arbitrary manipulation of spatial amplitude and phase using phase-only spatial light modulators," Sci. Rep.4, 7441, (2014) [doi: 10.1038/srep07441].

7. L. Wu, S. Cheng, and S. Tao, "Simultaneous shaping of amplitude and phase of light in the entire output plane with a phase-only hologram," Sci. Rep. 5, 15426, (2015) [doi: 10.1038/srep15426].

8. T. W. Clarket al., "Comparison of beam generation techniques using a phase only spatial light modulator," Opt. Express 24(6), 6249-6264 (2016) [doi:10.1364/OE.24.006249].

9. J. A. Daviset al., "Encoding amplitude information onto phase-only filters," Appl. Opt. 38(23), 50045013 (1999).

10. V. Arrizón, U. Ruiz, R. Carrada, and L. A. González, "Pixelated phase computer holograms for the accurate encoding of scalar complex fields," J. Opt. Soc. Am. A. 24(11), 3500-3507 (2007).

11. T. Sarkadi, Á. Kettinger, and P. Koppa, "Spatial filters for complex wavefront modulation,” Appl. Opt. 52(22), 5449-5454 (2013).

12. E. Bolducet al., "Exact solution to simultaneous intensity and phase encryption with a single phaseonly hologram,” Opt. Lett. 38(18), 3546- 3549 (2013).

13. T. Nobukawa, and T. Nomura, "Linear phase encoding for holographic data storage with a single phase-only spatial light modulator," Appl. Opt. 55(10), 2565-2573 (2016) [http://dx.doi.org/10.1364/AO.55.002565]. 
14. J. L. Martínez-Fuentes, and I. Moreno, "Random technique to encode complex valued holograms with on axis reconstruction onto phase-only displays," Opt. Express 26(5), 5875- 5893 (2018) [https://doi.org/10.1364/OE.26.005875].

15. R. W. Gerchberg and W. O. Saxton, "A Practical Algorithm for the Determination of Phase from Image and Diffraction Plane Pictures" Optik (Jena) 35, 237-246 (1972).

16. M. A. Seldowitz, J. P. Allebach, and D. W. Sweeney, "Synthesis of digital holograms by direct binary search," Appl. Opt. 26(14), 2788-2798 (1987) [https://doi.org/10.1364/AO.26.002788].

17. X. Li, J. Liu, J. Jia, Y. Pan, and Y. Wang, "3D dynamic holographic display by modulating complex amplitude experimentally," Opt. Express 21(18), 20577-20587 (2013) [https://doi.org/10.1364/OE.21.020577].

18. A. J. Cable, E. Buckley, P. Marsh, N. A. Lawrence, T. D. Wilkinson, and W. A. Crossland, "Realtime binary hologram generation for high-quality video projection applications," SID International Symposium Digest of Technical Papers, 35, 1431-1433 (2004).

19. P.W.M. Tsang, Y.-T. Chow, and T. -C. Poon, "Generation of phase-only Fresnel hologram based on down-sampling," Opt. Express 22(21), 25208-25214 (2014) [https://doi.org/10.1364/OE.22.025208].

20. P.W.M. Tsang, Y.T. Chow, and T.-C. Poon, "Generation of patterned-phase-only holograms (PPOHs)," Opt. Express 25(8), 9088-9093 (2017) [https://doi.org/10.1364/OE.25.009088].

21. O. Mendoza-Yero, G. Mínguez-Vega, and J. Lancis, "Encoding complex fields by using a phaseonly optical element," Opt. Lett. 39(7), 1740-1743 (2014) [http://dx.doi.org/10.1364/OL.39.001740].

22. J. Hu, C.-S. BRÈS, and C.-B. Huang, "Talbot effect on orbital angular momentum beams: azimuthal intensity repetition-rate multiplication," Opt. Lett. 43(16), 4033-4036 (2018) [https://doi.org/10.1364/OL.43.004033].

23. Y. Qi, C. Chang, and J. Xia, "Speckleless holographic display by complex modulation based on double-phase method," Opt. Express 24(26), 30368- 30378 (2016) [http://dx.doi.org/10.1364/OE.24.030368]. 
24. L. Gonget al., "Controllable light capsules employing modified Bessel-Gauss beams," Sci. Rep.6, 29001, (2016) [doi: 10.1038/srep29001].

25. C. Changet al., "Shaping of optical vector beams in three dimensions," Opt. Lett. 42(19), 3884-3887 (2017) [https://doi.org/10.1364/OL.42.003884].

26. T. Liet al., "Multifocus autofocusing Airy beam," J. Opt. Soc. Am. A. 34(9), 1530-1534 (2017) [https://doi.org/10.1364/JOSAA.34.001530].

27. Y. Zhaet al., "Elliptical Airy beam,” Appl. Opt. 57(23), 6717-6720 (2018) [https://doi.org/10.1364/AO.57.006717].

28. A. Lizanaet al., "Time fluctuations of the phase modulation in a liquid crystal on silicon display: Characterization and effects in the diffractive optics," Opt. Express 16(21), 16711-16722 (2008).

29. J. L. Martínezet al., “Analysis of multiple internal reflections in a parallel aligned liquid crystal on silicon SLM," Opt. Express 22(21), 25866-25879 (2014) [doi:10.1364/OE.22.025866].

30. S. Reichelt, "Spatially resolved phase-response calibration of liquid crystal-based spatial light modulators," Appl. Opt. 52(12), 2610-2617 (2013).

31. C. Lingel, T. Haist, and W. Osten, "Optimizing the diffraction efficiency of SLM-based holography with respect to the fringing field effect," Appl. Opt. 52(28), 6877-6883 (2013) [http://dx.doi.org/10.1364/AO.52.006877].

32. E. Ronzittiet al., "LCoSnematic SLM characterization and modeling for diffraction efficiency optimization, zero and ghost orders suppression," Opt. Express 20(16), 17843-17855 (2012).

33. J. E. Stockley, D. Subacius and S. A. Serati, "The Influence of the Inter-pixel Region in Liquid Crystal Diffraction Gratings," Liquid Crystal Displays II, SPIE 3635, (1999).

34. I. Yamaguchi, and T. Zhang, "Phase-shifting digital holography," Opt. Lett. 22(16), 1268-1270 (1997). 
Omel Mendoza-Yero is professor and researcher at the University Jaume I (UJI) in Spain. He is member of the Photonics Group (GROC-UJI) at UJI. He received his BS and PhD degrees in Physics from the University of Havana in 1998 and 2005, respectively. He is the author of more than 60 journal papers as well as several contributions to photonics congresses. His current research interests include ultrafast optics, diffractive optics, and beam shaping.

Miguel Carbonell-Leal received his BS degree in computer engineering and MS degree in artificial intelligence from the University Jaume I (UJI) in 2014 and 2015, respectively. Since 2015, he has been working toward PhD degree with the Photonics Group (GROC-UJI) at UJI. He is author of 7 research papers, and about 20 contributions to scientific congresses. 


\section{Caption List}

Fig. 1Examples of the design process of trivial phase elements $\alpha(x, y)$ obtained with sampling gratings having pixel-cells of $1 \times 1,2 \times 2$, and $4 \times 4$ pixels, respectively.

Fig. 2Off-axis optical setup used to investigate the effects of pixel crosstalk in double-phase method. Acronyms of included elements are NF (neutral filters), BE (beam expander), SLM (spatial light modulator), and L1 and L2 (refractive lenses).

Fig. 3Original amplitude pattern (a) and corresponding ones (b), (c), and (d) recorded with sampling gratings of $1 \times 1,4 \times 4$, and $10 \times 10$ pixels per cell, respectively.

Fig. 4Examples of two modified phase elements $\alpha_{M}(x, y)$ obtained after a convenient sampling process with a binary grating $M_{3}(x, y)$.

Fig. 5Original amplitude (a) and phase (b) images, and corresponding amplitude (c), (e), and (g) and phase (d), (f), and (h) images recorded for sampling gratings of $1 \times 1,2 \times 2$, and $5 \times 5$ pixels per cell, respectively. 


\title{
Diffraction-Based Phase Calibration of Spatial Light Modulators With Binary Phase Fresnel Lenses
}

\author{
Omel Mendoza-Yero, Gladys Mínguez-Vega, Lluís Martínez-León, Miguel Carbonell-Leal, \\ Mercedes Fernández-Alonso, Carlos Doñate-Buendía, Jorge Pérez-Vizcaíno, and Jesús Lancis
}

\begin{abstract}
We propose a simple and robust method to determine the calibration function of phase-only spatial light modulators (SLMs). The proposed method is based on the codification of binary phase Fresnel lenses (BPFLs) onto an SLM. At the principal focal plane of a BPFL, the focal irradiance is collected with a single device just able to measure intensity-dependent signals, e.g., CCD camera, photodiodes, power meter, etc. In accordance with the theoretical model, it is easy to extract the desired calibration function from the numerical processing of the experimental data. The lack of an interferometric optical arrangement, and the use of minimal optical components allow a fast alignment of the setup, which is in fact poorly dependent on environmental fluctuations. In addition, the effects of the zero-order, commonly presented in the diffraction-based methods, are drastically reduced because measurements are carried out only in the vicinity of the focal points, where main light contributions are coming from diffracted light at the BPFL. Furthermore, owing to the simplicity of the method, full calibration can be done, in most practical situations, without moving the SLM from the original place for a given application.
\end{abstract}

Index Terms-Calibration, diffractive optical elements (DOEs), spatial light modulator (SLM).

\section{INTRODUCTION}

$\mathbf{S}$ PATIAL light modulators (SLMs) can be regarded as excellent tools to manipulate at will the amplitude and phase of laser beams. They have been widely used to encode diffractive optical elements (DOEs) and to manipulate a variety of light properties, with important roles in linear/nonlinear microscopy [1], micro-processing of materials [2], spatial beam shaping and optical tweezers [3], wavefront sensors and adaptive optics [4], or pulse shaping [5], among many other applications.

At present, spatial light modulation can be carried out with the help of different devices. For instance, by using parallel aligned liquid crystal on silicon (LCoS) SLMs, with refresh rates in the order of few tens of $\mathrm{Hz}$ and phase-only modulation mode, the required dynamic range of most applications is reached [6]. Other devices, such as the digital micromirrors devices

Manuscript received September 30, 2015; revised January 8, 2016; accepted June 7, 2016. Date of publication June 14, 2016; date of current version September 13, 2016. This work was supported in part from MINECO under Grant FIS2013-40666-P, Generalitat Valenciana under Grants PROMETEO2012-021 and ISIC 2012/013, and Universitat Jaume I (P1-1B2012-55).

O. Mendoza-Yero, G. Mínguez-Vega, L. Martínez-León, M. CarbonellLeal, C. Doñate-Buendía, J. Pérez-Vizcaíno, and J. Lancis are with the Institute of New Imaging Technologies, Universitat Jaume I, Castelló 12071, Spain (e-mail: omendoza@uji.es; gminguez@ fca.uji.es; lluis.martinez@uji.es; a1095690@alumail.uji.es; cdonate@uji.es; jvizcain@uji.es; lancis@uji.es).

M. Fernández-Alonso is with the Department of Physics, University Jaume I, Castellón de la Plana, Spain (e-mail: fernande@fca.uji.es).

Color versions of one or more of the figures are available online at http://ieeexplore.ieee.org.

Digital Object Identifier 10.1109/JDT.2016.2580902
(DMDs), with refresh rates up to tens of $\mathrm{kHz}$ and amplitudemodulation mode, may approach real-time responses [7]. In addition, deformable mirrors offer the possibility to correct the wavefront of light beams. The calibration method proposed in this paper will be applied to phase-only SLMs, such as the commercially available parallel aligned LCoS modulators.

Former devices usually required complex calibration procedures. In the case of liquid crystal SLMs, complete calibration may consider the own SLM as a retarder-rotator system [8], which often exhibited a coupled phase and polarization modulation. In this context, theoretical expressions for the eigenvalues and the eigenvectors for twisted-nematic liquid-crystal displays as a function of the twist angle and the birefringence have been derived [9]. In this manuscript, authors also discuss techniques for achieving amplitude-only modulation, and coupled amplitude and phase modulation. Using another technique in which Jones matrices describing their polarization, a characterization of a reflective Holoeye LC-R 2500 SLM was also carried out [10], and applied in a holographic optical tweezers setup. Furthermore, a full characterization of an LCoS SLM, for phase-mostly modulation has been done, showing that polar decomposition of Muller matrices determines the polarization properties of the device [11].

The calibration process determines the phase response of liquid crystal SLMs as a function of a certain control parameter, e.g., the voltage signal applied to each pixel of the device. The relation between the output phase values and the input signal e.g., 256 grey levels contained in the displayed image, is the so-called calibration curve/function. The calibration process is a mandatory task before carrying out most practical implementation of SLMs within optical systems. Now, several types of calibration methods have been reported. In general, they can be separated into two main groups. One of them are the interferometric methods whereas, on the other part, we can find the diffractive-based ones. However, there are a remaining, but less widespread, set of calibration methods that cannot be included into the above groups.

The first group includes, but is not limited to, methods like the Young's fringes-derived phase characterization method [12]. In this case, the interference pattern is achieved by using a mask with two pinholes at the SLM plane, being the translation of the fringes at the Fourier plane affected by the phase modulation at each pinhole. Furthermore, the contrast of the fringe patterns depends on the similarity degree between the two pinholes. When a SLM shows nonuniform spatial response in its phase-versus-voltages curve, relatively complex interferometric setups are applied for calibration purposes. This is the case of 
the optical setup shown in [13], where a Michelson architecture, is composed, among other elements, of two polarizers, a piezo mirror, a beam splitter, and a CCD camera. The position dependent phase response of a transmissive SLM is measured and corrected in [14] with a Mach-Zehnder interferometer, including optical elements like polarizing beam splitters or two-lens Kepler-type imaging optics. A method, based on a shear interferometer, able to simultaneously measure the amplitude and phase modulation of a twisted liquid crystal display, in a relative simple manner, is shown in [15]. Here, it should be noted that apart from the inherent drawbacks related to the alignment of interferometers, as well as their high sensitivity to mechanical vibrations or air turbulences, above-mentioned calibration setups required a large number of optical elements.

Instead of interferometric, diffractive-based methods can be alternatively implemented for obtaining the calibration curves. In general these methods rely on the analysis of the diffraction field due to the interaction of light with certain multi-phase DOEs, previously encoded into the SLM [16]. To get the calibration curve, they employ phase retrieval algorithms. Other approaches simply quantify one of the parameters of the corresponding DOE in the far field, such as the intensity of the diffraction orders created by a two-level grating [17] or the visibility of a Fresnel image pattern [18]. Diffraction-based methods are in principle less sensitive to environmental disturbances, but in contrast they may be affected by residual intensity modulation, and/or by the discrepancies introduced by the zero order of diffraction associated with the non-diffracted light.

Other types of calibration methods, not included into the above-mentioned groups, use polarization set-ups. Basically, to obtain the calibration function, intensity measurements along cross-polarizers are performed [14]. The first polarizer rotates the input beam polarization at $45^{\circ}$ with respect to the aligned axis of the liquid crystal molecules. Then, a second polarizer is set at $-45^{\circ}$ with respect to the input polarization plane. As phase variations are converted into intensity fluctuations/phase shifts for calibration purposes, above configuration is known as intensity modulation. A complete characterization of a liquid crystal SLM is possible with a system including additional polarization elements [8], [19].

At this point, it is important to mention that accuracy of most phase characterization methods can be significantly influenced by unwanted physical phenomena associated with the performance of SLMs. For instance, it is well-known that temporal fluctuations of the liquid crystal molecular orientation as a function of time causes depolarization effects, deteriorating the diffraction efficiency of SLM [20]. In fact, when temporal fluctuations occur, the hypothesis of constant phase during measuring time is no longer valid. Another harmful effect that should originate e.g., non-linear phase modulation or coupled amplitude modulation, is related to the Fabry-Perot multiplebeam interference generated by the intrinsic layer structure of the liquid crystal device [21]. In this work, authors pointed out that, in cases of illumination of LCoS SLMs under angles other than normal incidence or/and phase modulation regimes higher than $2 \pi$, multiple reflection interference is increased. These drawbacks, as well as many other problems, including non-uniformities in the backplane [14] or fringing field effects [22], should be taken into account to a greater or lesser extent depending on the accurate measurements required for a given application. Furthermore, detailed studies of their consequences on some interferometric or diffraction-based phase calibration techniques have been reported [20]. Hence, the study of abovementioned effects is beyond of the scope of the present work.

In this manuscript we introduce an extraordinary simple and compact diffractive-based method for calibrating the phase response of a liquid crystal phase-only SLM. It is based on the measurement of the focal irradiance of binary phase Fresnel lenses (BPFLs) encoded into a phase-only SLM. Owing to circular symmetry of BPFLs, the measurement of the focal irradiance is carried out on-axis, which is usually of great convenience. Additionally, the influence of the zero order on the focal irradiance measurements is quite mitigated because of the preponderant diffraction nature of the collected light. Note that, in comparison with a Fourier hologram that uses a lens to focus the whole incoming light (modulated or not), Fresnel holograms given by BPFLs originate diffraction orders for which contribution of non-modulated light is negligible. On the other hand, the proposed diffraction-based optical setup can be less affected by environmental fluctuations than other two-arms interferometric arrangements used for the same purpose, with the exception of methods based on in-line interferometric setups that do not suffer from vibrations. As our method needs only to record the irradiance of a well spatially localized focal point, in principle, there is no need to use a measuring device with spatial resolution. That is, light intensity changes at the focal point can be collected with single-pixel detectors, e.g., photodiodes, power meters or even common spectrometers. Hence, the optical system is composed, apart from the SLM itself, only of a beam splitter and a light intensity-dependent measuring device. Furthermore, owing to the fact that intensity measurements are relative our calibrating method is generally quite forgiving to non-uniformity irradiance profiles, especially slowly varying changes.

The content of the manuscript is organized as follows. In the first section, we develop the theoretical model that supports the phase calibration method. In the second section, a commercially available SLM is employed to demonstrate the usefulness of the proposed phase calibration method. In the third section, a comparison of the obtained results with a similar off-axis method, characterized by the use of binary phase gratings, is carried out. Finally, in the last section, the main conclusions of our work are presented.

\section{Theory Underlying the CALIBRATION METHOD}

In this section, the theoretical basis of our method is demonstrated. In particular, we will show that the diffraction efficiency of a BPFL depends on the difference between its two constituent phase levels. A BPFL consists of a set of radically symmetric phase rings, which interchange their phase values between two possible levels $\phi_{1}$ and $\phi_{2}$. The rings are designed in such a way that optical path lengths of rays diffracted from adjacent zones differ by an integral multiple of a design wavelength $\lambda$. Within 
the framework of the paraxial approximation, the radius $r_{n}$ for the $n$th zone is given by $r_{n}^{2} \approx 2 n \lambda f$ [23]. Hence, BPFLs are optical elements periodic in $r^{2}$, with period $r_{1}^{2}$, and transmittance function $t(r)$ described by the Fourier series [24],

$$
\begin{aligned}
t(r)= & \frac{\exp \left(i \phi_{1}\right)+\exp \left(i \phi_{2}\right)}{2}+ \\
& +\sum_{\substack{m=-\infty \\
\text { modd }}}^{m=\infty} \frac{i\left[\exp \left(i \phi_{2}\right)-\exp \left(i \phi_{1}\right)\right]}{\pi m} \exp \left(\frac{i \pi m}{\lambda f} r^{2}\right)
\end{aligned}
$$

After comparing the phase given in Eq. (1), with the quadratic phase introduced by a well-known spherical lens, $\pi r^{2} /(\lambda f)$, one can realize that incident plane waves are diffracted in form of spherical waves with foci located at the axial positions $f_{m}=$ $r_{1}^{2} /(2 m \lambda)$. From Eq. (1), it is also apparent that the intensity of each focus can be calculated through the squared modulus of the corresponding coefficient in the Fourier series.

Neglecting reflection losses, we define the diffraction efficiency of a BPFL as the ratio between the integrated irradiance in the focus, and that of whole incident light. In mathematical terms, diffraction efficiency $\eta_{m}$ yields

$$
\eta_{m}=\left|\frac{i\left[\exp \left(i \phi_{2}\right)-\exp \left(i \phi_{1}\right)\right]}{\pi m}\right|^{2}=\left(\frac{2}{\pi m}\right)^{2} \operatorname{sen}^{2}\left(\frac{\phi_{2}-\phi_{1}}{2}\right) .
$$

From Eq. (2), it is apparent that the diffraction efficiency will depend on the selected focus, whereas the highest focal intensity (main focus) is obtained in the case $m=1$. In addition, the maximum focal efficiency is achieved when $\phi_{2}-\phi_{1}$ is equal to multiples of $\pi$. Equation (2) is the main theoretical result that supports our phase calibration method.

Owing to the fact that each gray level sent to the SLM is transformed into a given phase value, a complete set of BPFLs can be always constructed by selecting pairs of gray levels, e.g., the first one is zero and the second is set within the range from 1 to 255. In our implementation, the gray level 0 (corresponding to the phase value $\phi_{1}$ ) is fixed for all BPFLs. The combinations of the gray level 0 with the remaining ones, allow us to define 255 different BPFLs. Then, measurements of the integrated irradiance at a given focal plane, for the whole set of BPFLs is enough to obtain the experimental curve of the diffraction efficiency against gray level shifts. From the theory discussed here, it might be clear that above experimental curve is similar to the one predicted by Eq. (2), but now in terms of the phase shifts. To obtain the phase calibration curve e.g., the dependence of the gray levels with phase values, a suited fitting between experiment and theory is done.

\section{EXPERIMENTAL IMPLEMENTATION OF THE METHOD}

The phase calibration method proposed in this manuscript was tested by using the optical setup shown in Fig. 1. Before it impinges onto the beam splitter, the light beam emitted by a He-Ne laser (model 1135P from UNIPHASE, emitting at $632.8 \mathrm{~nm}$, and maximum output power of $10 \mathrm{~mW}$ ) is conveniently expanded with a commercial telescope (model

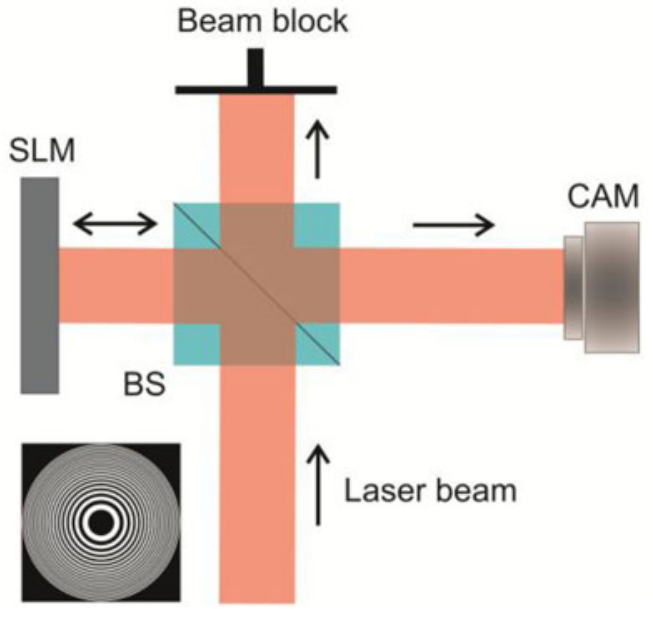

Fig. 1. Optical setup used for phase calibration of a phase-only SLM. The light from a He-Ne laser impinges onto the SLM after being reflected in the beam splitter. The camera collected the diffracted light at the focal plane of BPFLs encoded into the SLM.

EL-25-5X-B from THORLABS). This allows filling the whole area of the SLM display (Reflective PLUTO Phase Only SLM from HOLOEYE) which has a resolution of $1920 \times 1080$ pixels, with 8 micrometers of pixel pitch. The light beam reflected from the SLM is then transmitted through the beam splitter and sent to a conventional CCD camera (model UI-3480CP-M-GL from UEYE). Here, the use of a measuring device with spatial resolution is only motivated by the comparison (in the next section) of our method with an already established one. The intensity of the light at the CCD plane is always kept under the saturation level of the camera thanks to a previous attenuation of the light with neutral filters (not shown in the optical setup). In left bottom part of Fig. 1, the shape of a BPFL is also shown as an inset.

We encode a set of 255 different BPFLs into the SLM, constructed following the criteria described in the previous section. In our experiment, the position of the camera coincides with the main focal plane of BPFLs, which is located at $300 \mathrm{~mm}$ from the SLM plane. The focal images due to the diffraction of light by BPFLs are recorded with the CCD camera. The experiment is controlled with a Matlab software that takes less than 5 minutes to collect and process the data. We realize that this time duration is mainly limited by the refresh rate of the SLM (up to $60 \mathrm{~Hz}$ ), and the integration time set in the camera, whereas in less extent by the computer hardware.

From the recorded images, it can be obtained a signal proportional to the integrated irradiance of each focus. Hence, we sum the intensity of all pixels contained within the focal spot of the images. The results are normalized and shown in Fig. 2(a).

As the focal widths do not change when varying the phase of the BPFLs, both the number of pixels used to define the focal regions, and their locations are always the same. The focal region is defined by the $1 / e^{2}$ criterion for the beam width. The experimental curve of Fig. 2(a) shows a normalized integrated irradiance (or diffraction efficiency) behavior similar to the one described by Eq. (2), and represented in Fig. 2(b). Notice that, in Fig. 2(a) the irradiance depends on the different gray levels used 


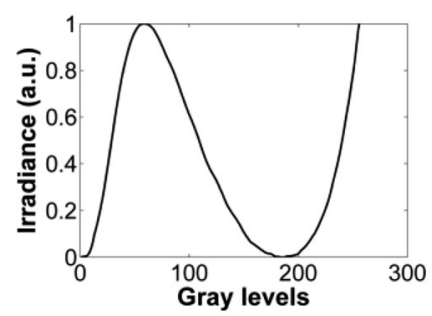

(a)

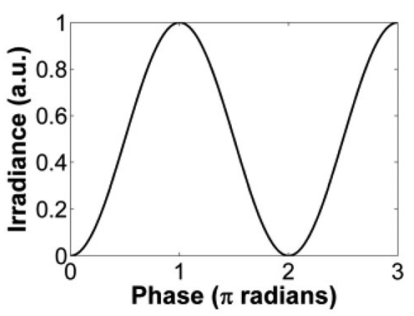

(b)
Fig. 2. Integrated irradiance at main focus plane of BPFLs in the region defined by the criterion, (a) experiment and (b) theoretical expression given in Eq. (2).

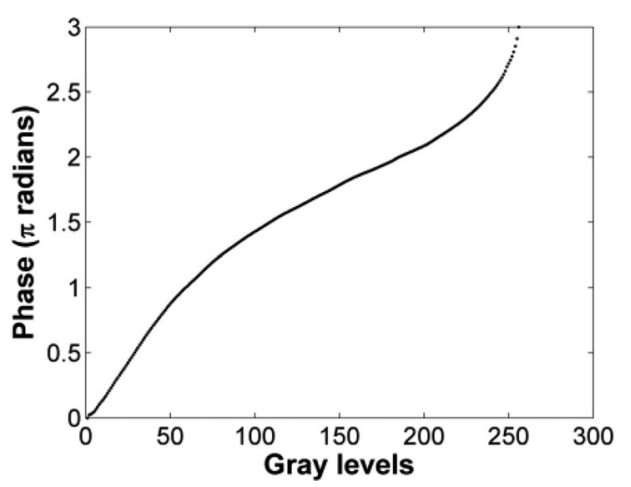

Fig. 3. Phase calibration function obtained with the proposed method, which is based on a set of BPFLs encoded into the SLM.

to encoded the BPFLs, whereas in Fig. 2(b) the irradiance varies with the phase values. The discrepancies between both curves will allow us to extract the information regarding the phase calibration. Here it should be mentioned that some extreme points in the original experimental curve did not coincide with the values 0 and/or 1 . This usually occurs, among other factors, due to non-homogeneities in the spatial phase response of the SLM or in the linear polarization of the laser beam. By using a discrete uniform distribution, we simulate the effect of nonuniform phase encoding of BPFLs on the integrated irradiance. Our simulation reproduced what happened in the experiment with a root mean square error less than $14 \%$. Therefore, to obtain the experimental curve shown in Fig. 2(a), the original raw data curve was numerically processed, following a standard procedure e.g., see section 5.2 of manuscript [25].

From the results of Fig. 2 one can determine the phase calibration curve of the SLM, that is, the dependence of phase values with the gray levels. To do that, phase shifts in Eq. (2) are expressed as a function of the normalized irradiance values. Then, normalized irradiance values, now taken from the experimental curve, are substituted into Eq. (2). Owing to the fact that the gray levels and phase values are linked by a common irradiance value, the phase calibration function can be generated. Note that, to get a real function the above process is carried out separately for each section of the curve defined between two local minima and maxima or vice versa. The phase calibration function is shown in Fig. 3. From this figure, it is clear that phase range of the SLM is extended approximately from 0 to $3 \pi$. The information extracted from Fig. 3 can be useful for

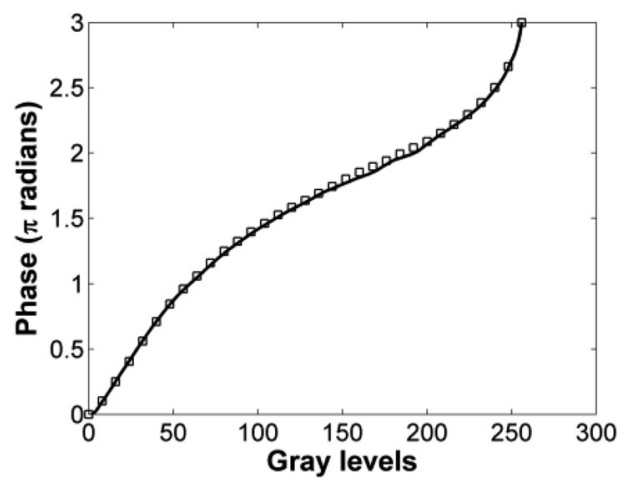

Fig. 4. Comparison between two phase calibration functions, (a) curve plotted with thick lines, obtained with the Ronchi grating method introduced in [17], (b) curve plotted with square symbols, obtained with our proposed method using BPFLs.

conveniently changing the look-up table, for example, to get a linear dependence between phase values and gray levels.

\section{VAlidation of The Method and Discussions}

In this section we use a well-established phase calibration method [17] to validate our proposal. For the experiment, we took advantage of the optical setup described in Fig. 1, including the same optical components. However, this time instead of BPFLs, a set of 255 Ronchi grating patterns are encoded into the SLM. They were designed with the equal gray level distribution as used before for BPFLs. The corresponding diffraction pattern at the Fourier plane is recorded again with the CCD camera, but including now an extra refractive lens of $380 \mathrm{~mm}$ focal length. The integrated irradiance was measured and numerically processed [25] for all diffraction gratings. To define the integration region, the $1 / e^{2}$ criterion for the beam width is applied. Then, following a similar procedure as being described in section III, the phase calibration function is determined. In Fig. 4, this curve is represented with thick solid lines. In addition, with square symbols, but using only 33 points, is plotted again corresponding phase calibration curve obtained with our method, i.e., equal to the one shown in Fig. 3.

After a visual inspection of Fig. 4 one can realize that phase calibration functions achieved with both methods are very similar. The root mean square error yields $6.2 \%$. Small discrepancies between both results can arise due to fluctuations in the laser beam position and/or energy, as well as differences caused by the spatial shape of focal points.

The last aspect may be better understood with the help of Fig. 5, where typical foci recorded with both methods are shown. In the method based on the implementation of Ronchi gratings, the focus is off-axis, because one looks for the first diffraction order of each grating, which typically have non circular symmetric spatial shape. In contrast, by using the method based on BPFLs, all axial diffraction orders (or foci) are on-axis, and consequently approach better to a circular symmetric pattern. Hence, the selection of the integration region may be slightly affected by the spatial shape of the focus.

So, from curves shown in Fig. 4 it is clear that our method offers reliable data for phase calibration purposes. In the labora- 


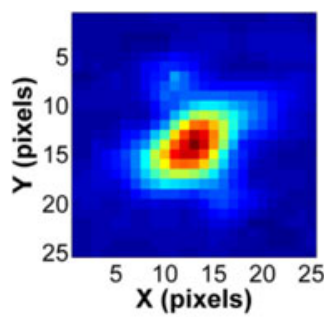

(a)

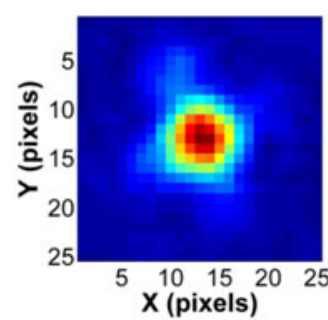

(b)
Fig. 5. Focal irradiance distribution of a pair of foci used for phase calibration, (a) with the method based on Ronchi gratings [17], (b) with the method based on BPFLs proposed in this manuscript.

tory, it has been also tested for different design parameters and conditions. In particular, for other periods of BPFLs, for measurements of the integrated irradiance at secondary focal planes, and for additional criteria regarding the definition of the integration region around a given focus. In all cases, the experiment gives results poorly dependent on the variable analyzed, with root mean square errors always less than $5 \%$. On the other hand, by means of numerical simulations we also tested the method for laser beams with Gaussian, and in general arbitrary, amplitude distributions at the SLM plane. The simulations basically show that phase calibration function does not change when modifying the amplitude of laser beams at the SLM plane. This conclusion might be expected since, from a practical point of view, relative and not absolute irradiance measurements are carried out.

The proposed method is mainly limited by the following aspects. We cannot correctly encode BPFLs with a main focal plane located below certain distance from the SLM. This critical distance can be calculated from the sampling criterion used to encode the minimum feature size of BPFLs. In our case, we assume that minimum feature size will be encoded with at least two pixels, which let us to a critical distance of approximately $150 \mathrm{~mm}$. Additionally, the proposed calibration method can give only a global value of the calibration function, which is unable to detect irregular phase responses associated with unexpected pixel behaviors or a non-uniform linear polarization of the laser beams, among other factors.

However, we believe that above-mentioned restrictions do not introduce severe problems in most applications, ranging from beam shaping to material processing or optical microscopy, being perhaps more critical for those situations where high quality spatial or temporal resolutions are needed. In these cases, the use of an SLM with increased spatial resolution, or the implementation of arrays of BPFLs may soften limitations.

\section{CONCLUSION}

In this manuscript, we propose an extremely compact phase calibration method based on irradiance measurements of the diffraction pattern originated by BPFLs at their focal planes. The simplicity of the method mainly relies on the small number of optical components needed for the calibration, which are limited to a common beam splitter, and an optical device able to measure light intensity changes. In fact, for optical layouts characterized by the use of reflective SLMs, having certain tilt with respect to the laser beam, the beam splitter for the calibration is also unnecessary. Hence, in many cases, the proposed method allows carrying out the calibration task without moving the SLM from the original position chosen for the application. In addition, due to its diffractive-based nature, the method is less affected by environmental changes than other calibration methods based on interferometric setups. For on-axis calibrations, the method generates focal irradiance distributions with circular symmetry, which can be convenient not only for phase calibration under quasi-monochromatic radiation, but also for simultaneously multi-wavelength calibration of broadband light sources by using an adequate spectrometer.

\section{REFERENCES}

[1] C. Maurer, A. Jesacher, S. Bernet, and M. Ritsch-Marte, "What spatial light modulators can do for optical microscopy," Laser Photon. Rev., vol. 5, pp. 81-101, 2011.

[2] Z. Kuang et al., "High throughput diffractive multi-beam femtosecond laser processing using a spatial light modulator," Appl. Surf. Sci., vol. 255, pp. 2284-2289, 2008.

[3] G. Sinclair et al., "Interactive application in holographic optical tweezers of a multi-plane Gerchberg-Saxton algorithm for three-dimensional light shaping," Opt. Express, vol. 12, pp. 1665-1670, 2004.

[4] J. Arines et al., "Measurement and compensation of optical aberrations using a single spatial light modulator," Opt. Express, vol. 15, pp. 1528715292,2007

[5] A. M. Weiner and I. Introduction, "Femtosecond pulse shaping using spatial light modulators," Rev. Sci. Instrum., vol. 71, pp. 1929-1960, 2000.

[6] G. Lazarev, A. Hermerschmidt, S. Krüger, and S. Osten, "LCOS spatial light modulators: Trends and applications," in Optical Imaging and Metrology: Advanced Technologies, W. Osten and N. Reingand, Eds. Hoboken, NJ, USA: Wiley, 2012.

[7] N. Savage, "Digital spatial light modulators," Nature Photon., vol. 3, pp. 170-172, 2009.

[8] V. Durán, J. Lancis, E. Tajahuerce, and Z. Jaroszewicz, "Equivalent retarder-rotator approach to on-state twisted nematic liquid crystal displays," J. Appl. Phys., vol. 99, 2006, Art. no. 113101.

[9] J. A. Davis, I. Moreno, and P. Tsai, "Polarization eigenstates for twistednematic liquid-crystal displays," Appl. Opt., vol. 37, pp. 937-945, 1998.

[10] J. Andilla, E. Martín-Bardosa, and S. Vallmitjana, "Prediction of phasemostly modulation for holographic optical tweezers," Opt. Commun., vol. 281, pp. 3786-3791, 2008.

[11] P. Clemente, V. Duran, Ll. Martínez-León, V. Climent, E. Tajahuerce, and J. Lancis, "Use of polar decomposition of Muller matrices for optimizing the phase response of liquid-crystal-on-silicon display," Opt. Express, vol. 16, pp. 1965-1974, 2008.

[12] A. Bergeron, J. Gauvin, F. Gagnon, D. Gingras, H. H. Arsenault, and M. Doucet, "Phase calibration and applications of liquid-crystal spatial light modulator," Appl. Opt., vol. 34, pp. 5133-5139, 1995.

[13] X. Xun and R. W. Cohn, "Phase calibration of nonuniform spatial light modulators," Appl. Opt., vol. 35, pp. 6400-6406, 2004.

[14] S. Reichelt, "Spatially resolved phase-response calibration of liquidcrystal-based spatial light modulators," Appl. Opt., vol. 52, pp. 2610-2617, 2013.

[15] F. P. Ferreira and M. S. Belsley, "Direct calibration of a spatial light modulator by lateral shearing interferometry," Opt. Express, vol. 18, pp. 7899-7904, 2010.

[16] D. Engstrom, G. Milewski, J. Bengtsson, and S. Galt, "Diffraction-based determination of the phase modulation for general spatial light modulators," Appl. Opt., vol. 45, pp. 7195-7204, 2006.

[17] Z. Zhang, G. Lu, and F. S. Yu, "Simple method for measuring phase modulation in liquid crystal televisions," Opt. Eng., vol. 33, pp. 30183022, 1994.

[18] Ll. Martinez-León, Z. Jaroszewicz, A. Kolodziejczk, V. Durán, E. Tajahuerce, and J. Lancis, "Phase calibration of spatial light modulators by means of Fresnel images", J. Opt. A: Pure Appl. Opt., vol. 11, p. $125405,2009$. 
[19] I. Moreno, P. Velásquez, C. R. Fernández-Pousa, M. M. Sánchez-López, and F. Mateos, "Jones matrix method for predicting and optimizing the optical modulation properties of a liquid-crystal display," J. Appl. Phys., vol. 94, pp. 3697-3702, 2003.

[20] A. Lizana et al., "Time fluctuations of the phase modulation in a liquid crystal on silicon display: Characterization and effects in the diffractive optics," Opt. Express, vol. 16, pp. 16711-16722, 2008.

[21] J. L. Martínez, I. Moreno, M. Sanchéz-López, A. Vargas, and P. GarcíaMartínez, "Analysis of multiple internal reflections in a parallel aligned liquid crystal on silicon SLM," Opt. Express, vol. 22, pp. 25866-25879, 2014.

[22] C. Lingel, T. Haist, and W. Osten, "Optimizing the diffraction efficiency of SLM-based holography with respect to the fringing field effect," Appl. Opt., vol. 52, pp. 6877-6883, 2013.

[23] S. Sinzinger and J. Jahns, Microoptics, 2nd ed., Hoboken, NJ, USA: Wiley, 2003, pp. 152-155.

[24] V. Moreno, J. F. Román, and J. R. Salgueiro, "High efficiency diffractive lenses: Deduction of kinoform profile," Amer. J. Phys., vol. 65, pp. 556$562,1997$.

[25] D. Engstrom, M. Persson, J. Bengtsson, and M. Goksor, "Calibration of spatial light modulators suffering from spatially varying phase response," Opt. Express, vol. 21, pp. 16086-16103, 2013.

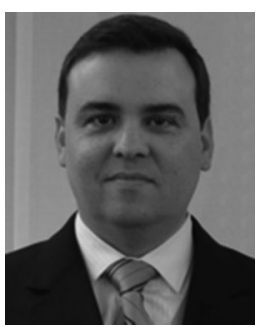

Omel Mendoza-Yero received the B.S. and Ph.D. degrees in physics from the University of Havana, Havana, Cuba, in 1998 and 2005, respectively.

From 1999 to 2002, he has been with the Optoelectronics Group, National Centre for Scientific Research in Cuba, working mainly on laser medicine projects. In 2003, he moved to the Laboratory of Laser Technology, Havana University to investigate on topics related to the development of equipments and technologies for laser processing of materials. Since 2005, he has been developing research activities on Ultrafast Optics at the Photonics Research Group, University Jaume I, Castellon, Spain. His current research interests include, but are not limited to pulse and beam shaping with spatial light modulators, non-linear effects induced by ultrashort pulses, and non-linear microscopy. $\mathrm{He}$ is author or co-author of more than 40 papers in peer reviewed scientific journals, as well as above 100 contributions to scientific congresses.

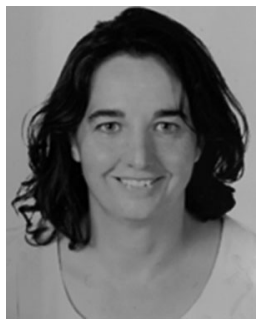

Gladys Mínguez-Vega received the B.Sc. and Ph.D. degrees in physics from the University of Valencia, Valencia, Spain, in 1997 and 2002, respectively.

She is an Associate Professor of Optics in the Universitat Jaume I of Castellón, Spain.

Her research interests include ultrafast optics, mainly in management of femtosecond beams with DOEs, both static and dynamic ones, for applications in nonlinear optics, material microprocessing, highspeed microscopy, and nanoparticle fabrication. She has developed part of her research as a Visiting Scientist in the Purdue University, Fernuniversität in Hagen, Germany, and Applied Optics Institute, Poland. She has published two book chapters, more than 50 journal articles, and more than 80 conference papers ( 10 of them by invitation).

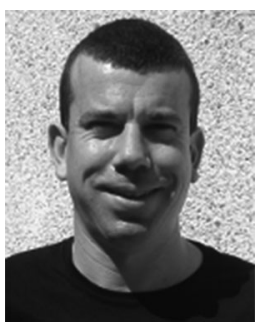

Lluís Martínez-León received the B.Sc. and Ph.D. degrees in physics from the University of Valencia, Valencia, Spain, in 1997 and 2002, respectively.

$\mathrm{He}$ is an Assistant Professor at Universitat Jaume I in Castelló (Spain), where he is a Member of the Photonics Research Group, GROC•UJI. His current research interests lie in the areas of single-pixel cameras, interferometry and digital holography, microscopy, super-resolution, image processing, whitelight systems, spatial-light modulators, and femtosecond optics.

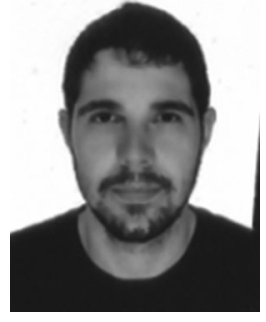

Miguel Carbonell-Leal received the B.S. degree in computer engineering and the M.S. degree in artificial intelligence, both from the University Jaume I of Castellon in 2014 and 2015, respectively. Since 2015, he has been working toward Ph.D. degree with Group GROC•UJI, Department of Physics, University Jaume I of Castellon.

$\mathrm{He}$ is co-author of 1 research journal, and 12 contributions to scientific congresses.

Mr. Carbonell-Leal is a Member of the Optical Society of America and the international society for optics and photonics. He is the President of Group of Optics Castellón SPIE student chapter.

Mercedes Fernández-Alonso received the degree in Physics (specialty of Fundamental Physics) from the University of Cantabria, Santander, Spain, in 1982. She received the doctorate degree in physics (in the field of Elementary Particle Physics) from the University of Cantabria, Santander, Spain, in 1989. There followed a period of postdoctoral training during 1990 to 1991 at the European Center for Particle Physics in Switzerland.

In the academic year 1991-1992, she joined the Universitat Jaume I of Castellón, Department of Physics and at present she works there as Associate Professor. Her research interests include Optics and Photonics, working in Imaging Science and Ultrafast Optics. She is coauthor of more than 70 research papers, and 50 communications in national or international conference meetings. She is Member of the Real Sociedad Española de Física.

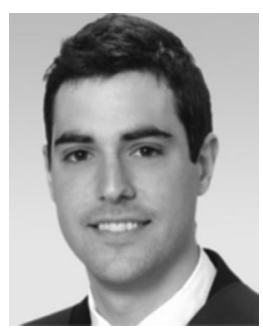

Carlos Doñate-Buendía received the degree in physics and the M.Sc. degree in advanced physics in the specialty of photonics, both from the University of Valencia in 2014 and 2015, respectively. Since 2016, he has been working toward the Ph.D. degree with Optics Group GROC-UJI, Department of Physics, University Jaume I from Castellon. He is co-author of 10 contributions to scientific congresses.

Jesús Lancis is a Full Professor of optics at University Jaume I in Castellón, where he currently leads the Photonics Research Group "Grupo de Investigación de Óptica de Castellón, GROC.UJI" (https://sites.google. com/a/uji.es/photonics-uji/). The group is engaged in basic research to deliver on the promise of photonics breakthroughs. His laboratory is focused on digital control of light beams with microstructure masks codified onto megapixel spatial light modulators. This work is aimed to overcome the limitations of current photonics technologies for healthcare and laser manufacturing processes. He has been the key researcher in more than 10 research programs at the local, regional, national, and international level. He has contributed more than 115 research papers in major photonics journals located in the first positions of the ISI Journal Citation Reports (cited 1279 times, h-index 19 Web of Science). He has presented more than 40 invited or keynote talks on photonics in different meetings organized by well-established scientific societies (OSA, IEEE, EOS, EPS , ... ) and supervised four early stage researchers that obtained the Ph.D. degree in physics.

Dr. Lancis is a Senior Member of the Optical Society of America and a Regular Member of the European and the Spanish Optical Societies. He leads the Doctoral Training Center of University Jaume I, where he is strongly committed to promote excellence and good practice in training of early stage researchers.

Jorge Pérez-Vizcaíno's photograph and biography not available at the time of publication. 


\title{
Diffractive control of 3D multifilamentation in fused silica with micrometric resolution
}

\author{
Omel Mendoza-Yero, ${ }^{1,{ }^{*}}$ Miguel Carbonell-Leal, ${ }^{1}$ \\ CARlos doñate-BuendíA, ${ }^{1}$ Gladys MíngueZ-VegA, ${ }^{1}$ \\ AND JESÚS LANCIS ${ }^{1}$ \\ ${ }^{I}$ GROC•UJI, Institute of New Imaging Technologies, Universitat Jaume I, 12071-Castelló, Spain \\ *omendoza@uji.es
}

\begin{abstract}
We show that a simple diffractive phase element (DPE) can be used to manipulate at will the positions and energy of multiple filaments generated in fused silica under femtosecond pulsed illumination. The method allows obtaining three-dimensional distributions of controlled filaments whose separations can be in the order of few micrometers. With such small distances we are able to study the mutual coherence among filaments from the resulted interference pattern, without needing a two-arm interferometer. The encoding of the DPE into a phase-only spatial light modulator (SLM) provides an extra degree of freedom to the optical set-up, giving more versatility for implementing different DPEs in real time. Our proposal might be particularly suited for applications at which an accurate manipulation of multiple filaments is required.
\end{abstract}

(C)2016 Optical Society of America

OCIS codes: (070.6120) Spatial light modulators; (190.0190) Nonlinear optics; (190.7110) Ultrafast nonlinear optics; (320.6629) Supercontinuum generation.

\section{References and links}

1. W. R. Zipfel, R. M. Williams, and W. W. Webb, "Nonlinear magic: multiphoton microscopy in the biosciences," Nat. Biotechnol. 21(11), 1369-1377 (2003).

2. R. Meesat, H. Belmouaddine, J. F. Allard, C. Tanguay-Renaud, R. Lemay, T. Brastaviceanu, L. Tremblay, B. Paquette, J. R. Wagner, J. P. Jay-Gerin, M. Lepage, M. A. Huels, and D. Houde, "Cancer radiotherapy based on femtosecond IR laser-beam filamentation yielding ultra-high dose rates and zero entrance dose," Proc. Natl. Acad. Sci. U.S.A. 109(38), E2508-E2513 (2012).

3. R. R. Gattass and E. Mazur, "Femtosecond laser micromachining in transparent materials," Nat. Photonics 2(4), 219-225 (2008).

4. V. I. Klimov and D. W. McBranch, "Femtosecond high-sensitivity, chirp-free transient absorption spectroscopy using kilohertz lasers," Opt. Lett. 23(4), 277-279 (1998).

5. G. Cerullo and S. De Silvestri, "Ultrafast optical parametric amplifiers," Rev. Sci. Instrum. 74(1), 1-18 (2003).

6. J. J. Macklin, J. D. Kmetec, and C. L. Gordon 3rd, "High-order harmonic generation using intense femtosecond pulses," Phys. Rev. Lett. 70(6), 766-769 (1993).

7. A. M. Weiner, "Femtosecond pulse shaping using spatial light modulators," Rev. Sci. Instrum. 71(5), 1929-1960 (2000).

8. S. Hasegawa, Y. Hayasaki, and N. Nishida, "Holographic femtosecond laser processing with multiplexed phase Fresnel lenses," Opt. Lett. 31(11), 1705-1707 (2006)

9. L. Martínez-León, P. Clemente, E. Tajahuerce, G. Mínguez-Vega, O. Mendoza-Yero, M. Fernández-Alonso, J. Lancis, V. Climent, and P. Andrés, "Spatial-chirp compensation in dynamical holograms reconstructed with ultrafast lasers," Appl. Phys. Lett. 94(1), 011104 (2009).

10. S. H. Shim, D. B. Strasfeld, E. C. Fulmer, and M. T. Zanni, "Femtosecond pulse shaping directly in the mid-IR using acousto-optic modulation," Opt. Lett. 31(6), 838-840 (2006).

11. F. Verluise, V. Laude, J. P. Huignard, P. Tournois, and A. Migus, "Arbitrary dispersion control of ultrashort optical pulses with acoustic waves," J. Opt. Soc. Am. B 17(1), 138-145 (2000).

12. C. Y. Chang, L. C. Cheng, H. W. Su, Y. Y. Hu, K. C. Cho, W. C. Yen, C. Xu, C. Y. Dong, and S. J. Chen, "Wavefront sensorless adaptive optics temporal focusing-based multiphoton microscopy," Biomed. Opt. Express 5(6), 1768-1777 (2014).

13. B. Mills, M. Feinaeugle, C. L. Sones, N. Rizvi, and R. W. Eason, "Sub-micron-scale femtosecond laser ablation using a digital micromirror device," J. Micromech. Microeng. 23(3), 035005 (2013).

14. J. N. Yih, Y. Y. Hu, Y. D. Sie, L. C. Cheng, C. H. Lien, and S. J. Chen, "Temporal focusing-based multiphoton excitation microscopy via digital micromirror device," Opt. Lett. 39(11), 3134-3137 (2014).

15. O. Mendoza-Yero, V. Loriot, J. Pérez-Vizcaíno, G. Mínguez-Vega, J. Lancis, R. de Nalda, and L. Bañares, "Programmable quasi-direct space-to-time pulse shaper with active wavefront correction," Opt. Lett. 37(24), 5067-5069 (2012). 
16. J. Pérez Vizcaíno, O. Mendoza-Yero, R. Borrego-Varillas, G. Mínguez-Vega, J. R. Vázquez de Aldana, and J. Láncis, "On-axis non-linear effects with programmable Dammann lenses under femtosecond illumination," Opt. Lett. 38(10), 1621-1623 (2013).

17. G. Mínguez-Vega, C. Romero, O. Mendoza-Yero, J. R. Vázquez de Aldana, R. Borrego-Varillas, C. Méndez, P. Andrés, J. Lancis, V. Climent, and L. Roso, "Wavelength tuning of femtosecond pulses generated in nonlinear crystals by using diffractive lenses," Opt. Lett. 35(21), 3694-3696 (2010).

18. R. Borrego-Varillas, J. Perez-Vizcaino, O. Mendoza-Yero, G. Minguez-Vega, J. R. Vázquez de Aldana, and J. Lancis, "Controlled multibeam supercontinuum generation with a spatial light modulator," IEEE Photonics Technol. Lett. 26(16), 1661-1664 (2014).

19. A. Couairon and A. Mysyrowicz, "Femtosecond filamentation in transparent media," Phys. Rep. 441(2-4), $47-$ 189 (2007)

20. J. M. Dudley and S. Coen, "Coherence properties of supercontinuum spectra generated in photonic crystal and tapered optical fibers," Opt. Lett. 27(13), 1180-1182 (2002).

21. I. Zeylikovich and R. R. Alfano, "Coherence properties of the supercontinuum source," Appl. Phys. B 77(2-3), 265-268 (2003).

22. C. Corsi, A. Tortora, and M. Bellini, "Mutual coherence of supercontinuum pulses collinearly generated in bulk media," Appl. Phys. B 77(2-3), 285-290 (2003).

23. C. Corsi, A. Tortora, and M. Bellini, "Generation of a variable linear array of phase-coherent supercontinuum sources," Appl. Phys. B 78(3-4), 299-304 (2004).

24. R. Borrego-Varillas, J. Pérez-Vizcaíno, O. Mendoza-Yero, J. R. Vázquez de Aldana, G. Mínguez-Vega, and J. Lancis, "Dynamic control of interference effects between optical filaments through programmable optical phase modulation," J. Disp. Technol. (to be published).

25. C. Romero, R. Borrego-Varillas, A. Camino, G. Mínguez-Vega, O. Mendoza-Yero, J. Hernández-Toro, and J. R. Vázquez de Aldana, "Diffractive optics for spectral control of the supercontinuum generated in sapphire with femtosecond pulses," Opt. Express 19(6), 4977-4984 (2011).

26. R. Borrego-Varillas, C. Romero, O. Mendoza-Yero, G. Mínguez-Vega, I. Gallardo, and J. R. Vázquez de Aldana, "Femtosecond filamentation in sapphire with diffractive lenses," J. Opt. Soc. Am. B 30(8), 2059-2065 (2013).

27. A. Camino, Z. Hao, X. Liu, and J. Lin, "Control of laser filamentation in fused silica by a periodic microlens array," Opt. Express 21(7), 7908-7915 (2013).

28. O. Mendoza-Yero, G. Mínguez-Vega, and J. Lancis, "Encoding complex fields by using a phase-only optical element," Opt. Lett. 39(7), 1740-1743 (2014).

29. J. A. Davis and D. M. Cottrell, "Random mask encoding of multiplexed phase-only and binary phase-only filters," Opt. Lett. 19(7), 496-498 (1994).

30. C. Maurer, S. Khan, S. Fassl, S. Bernet, and M. Ritsch-Marte, "Depth of field multiplexing in microscopy," Opt. Express 18(3), 3023-3034 (2010).

31. C. Iemmi, J. Campos, J. C. Escalera, O. López-Coronado, R. Gimeno, and M. J. Yzuel, "Depth of focus increase by multiplexing programmable diffractive lenses," Opt. Express 14(22), 10207-10219 (2006).

32. R. Di Leonardo, F. Ianni, and G. Ruocco, "Computer generation of optimal holograms for optical trap arrays," Opt. Express 15(4), 1913-1922 (2007).

33. D. Milam, "Review and assessment of measured values of the nonlinear refractive-index coefficient of fused silica," Appl. Opt. 37(3), 546-550 (1998).

34. N. T. Nguyen, A. Saliminia, W. Liu, S. L. Chin, and R. Vallée, "Optical breakdown versus filamentation in fused silica by use of femtosecond infrared laser pulses," Opt. Lett. 28(17), 1591-1593 (2003).

\section{Introduction}

Extremely short temporal light events can be regarded as excellent tools for accessing nonlinear optical effects, i.e., self-phase modulation, self-focusing, or plasma generation, due to the combination of spatially focused and femtosecond time scale pulsed light. It is wellknown that a suited control over the spatial and temporal properties of ultrashort pulses allows manipulating non-linear phenomena for developing multiple tasks, including two-photon microscopy [1], cancer therapy [2], micro-processing of materials [3], or non-linear spectroscopy [4]. Other settle-down applications i.e., for getting the initial seed in ultrafast optical parametric amplifiers [5], or for synthesizing high harmonics [6] have also been reported. Here, it is apparent that setting specific parameters to ultrashort pulses can be hard to achieve due to several unwanted effects such as temporal dispersion or spatial phase aberrations. In the temporal and/or spatial domains, pulse parameters can be changed in realtime by using optical devices like liquid crystal SLMs [7-9], acoustic-optics crystals $[10,11]$ deformable mirrors [12] or digital micromirror devices (DMDs) $[13,14]$.

On the other hand, the use of diffractive optics has demonstrated its capability to provide not only compact temporal pulse shapers [15], but also to manipulate nonlinear optical phenomena in the axial $[16,17]$, as well as in the transversal direction of the pulse propagation [18]. In this context, we will focus on filamentation [19], which is basically a non-linear 
propagation phenomenon that is extended a distance longer than the Rayleigh range associated with the pulse. It is originated due to the balance of two main processes, pulse focusing by Kerr effect and defocusing caused by the plasma. Regarding this topic, the coherent nature of the filaments [20] has been investigated by means of several optical setups/devices such as a diffraction-grating-based interferometer [21], collinear geometries with time-delayed pulses [22], variable linear arrays of supercontinuum sources [23] or programmable liquid crystal SLMs [24]. In addition, the filamentation process in fused silica generated under femtosecond illumination has been studied by means of diffractive lenses [24-26]. In particular, conventional arrays of diffractive lenses have been implemented as a tool to generate multiple and predefined filaments in fused silica [24, 27]. At this point, we want to note that the utilization of a conventional array of diffractive lenses for multifilamentation has some drawbacks. The first one is related to the impossibility to bring filaments closer to a distance smaller than twice the physical radius of a lens without using additional optics (assuming arrays of equal lenses). The second drawback comes from the apparent reduction of the numerical aperture of lenses with respect to the numerical aperture of the optical system because of the array implementation itself. Furthermore, the different spatial locations of lenses within an array cause the corresponding focal energies to strongly depend on the initial irradiance distribution of the light source onto the plane of the lens array.

In this contribution we experimentally demonstrate a diffractive-based method to generate arbitrary three-dimensional distributions of filaments in fused silica with accurate control over the spatial locations of filaments. The filaments are originated after focusing femtosecond laser pulses into a fused silica by using a single DPE. The encoding of the DPE into phaseonly liquid crystal SLM gives additional degrees of freedom to the proposed method, allowing for a dynamic and more versatile operation. In addition, by modulating the phase functions of the lenses we can vary their diffraction efficiency, so modifying the amount of energy employed to produce each filament. Furthermore, due to the characteristics of the encoding method, it is easy to generate filaments with lateral separations in the order of few micrometers. Hence, it will be relatively simple to study the mutual coherence among filaments without using two-arm interferometers or any additional optical components.

The content of the manuscript is organized as follows. In section 2, details of the encoding method are given. In section 3, with the help of a femtosecond laser source and a commercially available SLM, several multifilamentation processes by means of DPEs are experimentally demonstrated. In section 4, we show the usefulness of DPEs to study the mutual coherence among filaments contained within two different spatial distributions. Finally, in section 5 the main conclusions of our work are presented.

\section{Basics of the encoding method}

In this manuscript a spatially multiplexed procedure is used to encode a set of diffractive lenses into a unique $\operatorname{DPE}(x, y)$. With this purpose, the phase information $\Omega_{n}(x, y)=\bmod _{2 \pi}\left\{\pi\left[\left(x-x_{0}\right)^{2}+\left(y-y_{0}\right)^{2}\right] /\left(f_{n} \lambda_{0}\right)\right\}$ corresponding to $N$ different off-axis Fresnel lenses, where $n=1 \ldots N$, is sampled with $N$ complementary spatially uniform masks $M_{n}(x, y)$ such as $\sum_{n=1}^{N} M_{n}(x, y)=1$. The function $\bmod _{2 \pi}$ holds for the module $2 \pi$ operation, $f_{n}$ is the focal length of the $n$th lens for the central wavelength of the light source $\lambda_{0}$, and variables $x$ and $y$ represent Cartesian transverse coordinates. From the above expression, each individual filament located at arbitrary transverse coordinates $x_{0}, y_{0}$ within the fused silica will be originated by the focused light associated with an off-axis kinoform lens also centered at $x_{0}, y_{0}$. In mathematical terms, the resulted $D P E(x, y)$ can be expressed as:

$$
\operatorname{DPE}(x, y)=M_{1}(x, y) \Omega_{1}(x, y)+\ldots+M_{N}(x, y) \Omega_{N}(x, y)
$$

In order to get further insight into the implementation of this encoding method, we include here a dummy example, see Fig. 1. In the first column of Fig. 1 the two-dimensional binary 
masks $M_{n}(x, y)$ employed for the sampling process are given. The square gray or black zones within these masks have values one or zero, respectively. In practice, each zone will correspond to one pixel of the SLM. For this example the phase functions $\Omega_{n}(x, y)$ do not represent lenses, but are substituted by the simple patterns shown in the second column of Fig. 1. In the right part of Fig. 1, the $\operatorname{DPE}(x, y)$ assessed from the sum of sampled functions $\Omega_{n}(x, y)$, as indicated in Eq. (1), is shown. Hence, it is clear that Fresnel lenses are encoded with different sets of pixels of the SLM. This allows for a separate manipulation of the position and energy coupled into each focus.

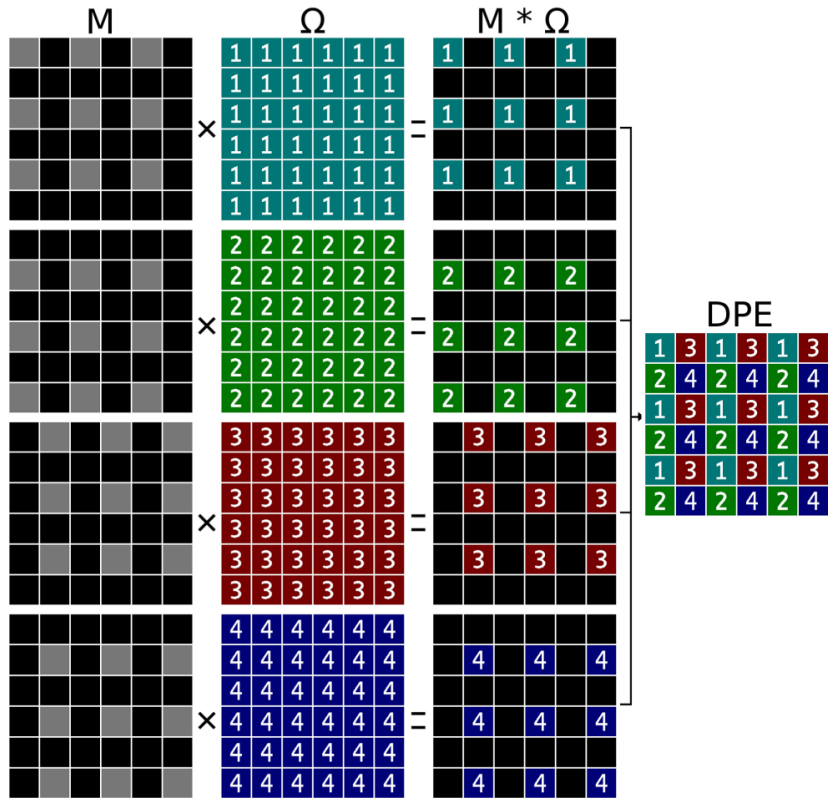

Fig. 1. Dummy example of the encoding method by steps: binary masks (left column), functions to be encoded (middle column), sampled functions (right column), and constructed DPE (right-part).

Note that, the applicability of this encoding method depends on the accuracy of the sampling process, which is directly linked to the pixel width. At this point, we point out that a good sampling process should allows reconstructing the original function by simple extrapolation. So, the number of lenses that can be encoded with this method depends on the available pixel width. For instance, in our case we are able to encode up to 9 lenses with a focal length of $245 \mathrm{~mm}$ and a pixel width of $8 \mu \mathrm{m}$. Furthermore, for a given pupil extension the lower the pixel width the greater the energy at the focal point. In this context, it can be demonstrated that the diffraction efficiency decreases quadratically with an increasing number of the superposed Fresnel lenses.

In the literature, similar strategies for spatially multiplexed Fresnel lenses have been reported [28-32]. For instance, it can be mentioned a method based on a random sampling of the phase called random mask encoding [31], or another characterized by the application of the weighted Gerchberg-Saxton algorithm [32]. In this manuscript we select a strategy that allows us to carry out a uniform sampling of the phase onto the SLM display. The main reason for doing that is obtaining similar amounts of energy at the focal points of Fresnel lenses even when the laser irradiance at the SLM plane has not a uniform distribution. In addition, as we will show later such a spatial multiplexed of lenses is very useful to achieve independent and precise control of the multifilamentation process in bulk. 


\section{Multifilamentation with DPEs}

To experimentally show the ability of DPEs to manipulate at will some parameters of multiple filaments developed in fused silica, we have constructed the optical setup shown in Fig. 2. The DPEs are constructed following the procedure described in section 2. The light emitted by a Ti: Sapphire femtosecond laser is used as pulsed illumination source. The output pulses are about $30 \mathrm{fs}$ intensity full width at half maximum, with $1 \mathrm{kHz}$ repetition rate, centered at $\lambda_{0}=800 \mathrm{~nm}$ with an approximate energy per pulse of $800 \mu \mathrm{J}$. Before it impinges into the liquid crystal SLM (Reflective PLUTO Phase Only SLM from HOLOEYE), the light is spatially magnified with the help of a 4X reflective optical beam expander (BE04R from Thorlabs). This magnification allows the light to fill the whole area of the liquid crystal display. After that, the light is sent to the SLM via a pellicle beam splitter (BP145B2 from Thorlabs).

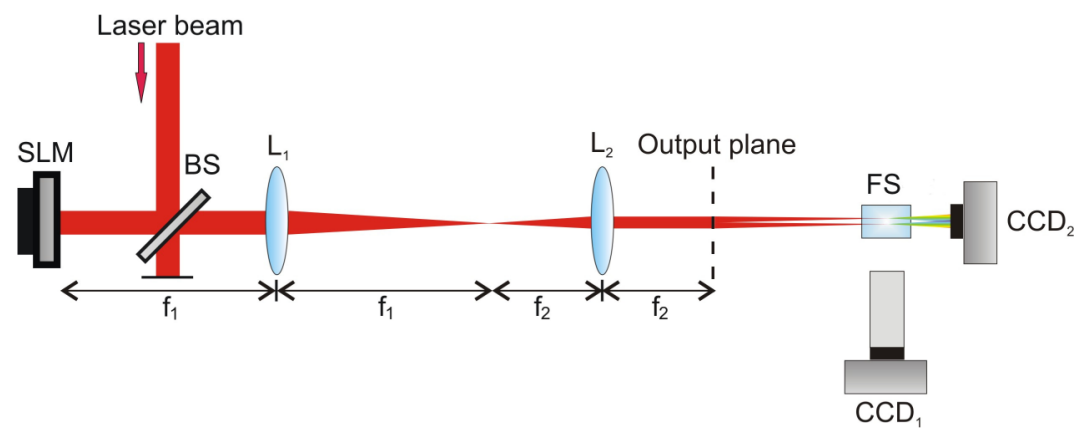

Fig. 2. Experimental setup for the generation of multiple filaments in fused silica with arbitrary spatial distribution and controlled energy by using a single diffractive phase element encoded into a phase-only SLM.

In order to get access to regions very close to the DPE, we form an image of the liquid crystal display with the help of a $4 \mathrm{f}$ optical system. This optical system is composed of a couple of lenses with focal lengths $f_{1}=300 \mathrm{~mm}$ and $f_{2}=150 \mathrm{~mm}$. The above combination of lenses decreases by a factor of two the transversal extension of the DPE at the output plane of our imaging system. Accordingly, the magnification of the imaging system in the axial direction is $1 / 4$. After the output plane of the imaging system, the pulse focuses towards the entrance face of a $10 \mathrm{~mm}$ thick fused silica (denoted as FS in Fig. 2), originating a spatial distribution of filaments inside the sample. As the light is focused with the DPE encoded into the SLM, non-diffracted light (e.g., zero order) does not contribute to the filament formation. Aside, a microscopy objective ( $25 \mathrm{~mm} / 0.15 \mathrm{NA}$ from Ealing) is used to form an image of the filaments with a magnification of 0.85 onto a CCD camera (model UI-1540SE-M-GL, with resolution $1280 \times 1024$ and pixel width $5.2 \mu \mathrm{m}$ from UEYE). Here, we conveniently designed the DPEs to generate spatial distributions of filaments, all of them contained in planes parallels to the plane of the CCD camera. This ensures that, for a given set of experimental parameters i.e., fixed distances among filaments or specific focal lengths for diffractive lenses, all filaments can be recorded at once with the CCD camera. In addition to the above-mentioned camera, another CCD camera (model A102fc, with resolution $1388 \times 1038$ and pixel width $6.45 \mu \mathrm{m}$ from Basler) is placed after the rear face of the fused silica in a plane perpendicular to the propagation direction of the filaments, see Fig. 2. This second camera is used to record images originated by the interference of multiple filaments as we will later explain in section 4.

In the present experiment, the focal length of diffractive lenses for the central wavelength of the pulse, $\lambda_{0}$, is $245 \mathrm{~mm}$. However, owing to the $1 / 4$ axial magnification of the $4 \mathrm{f}$ optical system, all foci arise in a transversal plane located $61.25 \mathrm{~mm}$ away from the output plane. In 
addition, the focal energy $E_{\text {focal }}$ per diffractive lens is approximately $1.4 \mu \mathrm{J}$. This energy was determined from the measuring of the average power $A_{\text {power }}$ of the pulse at the output plane, just after an iris that fits the pupil of the optical system in this plane. The iris eliminates undesired light reflected from zones of the SLM display outside the pupil. Using the above parameters and taking into account the repetition rate of the laser $R_{\text {rate }}$, and the theoretical values for the fill factor $F_{\text {factor }}$ and diffraction efficiency of our SLM $D_{\text {efficiency }}$, the focal energy was calculated by the expression $E_{\text {focal }}=A_{\text {power }} F_{\text {factor }} D_{\text {efficiency }} /\left(N_{\text {lenses }} R_{\text {rate }}\right)$, where $N_{\text {lenses }}$ holds for the number of lenses encoded into the DPE. At this point, $E_{\text {focal }}$ can be compared with the energy necessary for generating filamentation. The filamentation regime is achieved if the input power exceeds the critical power obtained from the balance between Kerr self-focusing and plasma defocusing, $P_{c r}=3.77 \lambda_{0}^{2} /\left(8 \pi n_{0} n_{2}\right)=2.5 \mathrm{MW}$ where $n_{0}=1.51$ is the refractive index of fused silica for $\lambda_{0}$, and $n_{2}=2.48 \cdot 10^{-20} \mathrm{~m}^{2} / \mathrm{W}$ is the nonlinear refractive index [33]. Hence, the energy per pulse needed for filamentation can be estimated $\left(E_{\text {focal } \min }=0.14 \mu \mathrm{J}\right)$. This means that if all the available energy $E_{\text {focal }}$ is employed for nonlinear processes, up to 10 additional filaments per encoded lens could be generated. In this context, it has been also shown [34] that for large focal lengths, the threshold energy for filamentation decreases as the corresponding one for the optical breakdown increases. For the parameters used in our experiments, e.g., $f=245 \mathrm{~mm}$, and $E_{\text {focal }}=1.4 \mu \mathrm{J}$, the filamentation process takes place far away from the optical breakdown, avoiding in this manner modification of the optical parameters or damage of the fused silica. Please, note that for our experimental conditions the size of the filaments $(500 \mu \mathrm{m})$ is approximately seven times bigger than the Rayleigh range ( $73 \mu \mathrm{m})$ calculated in the linear propagation regime.

In Fig. 3, recorded images corresponding to four different spatial distributions of filaments within the fused silica are shown. As expected, each distribution of filaments is generated by focusing the light with a specific DPE.
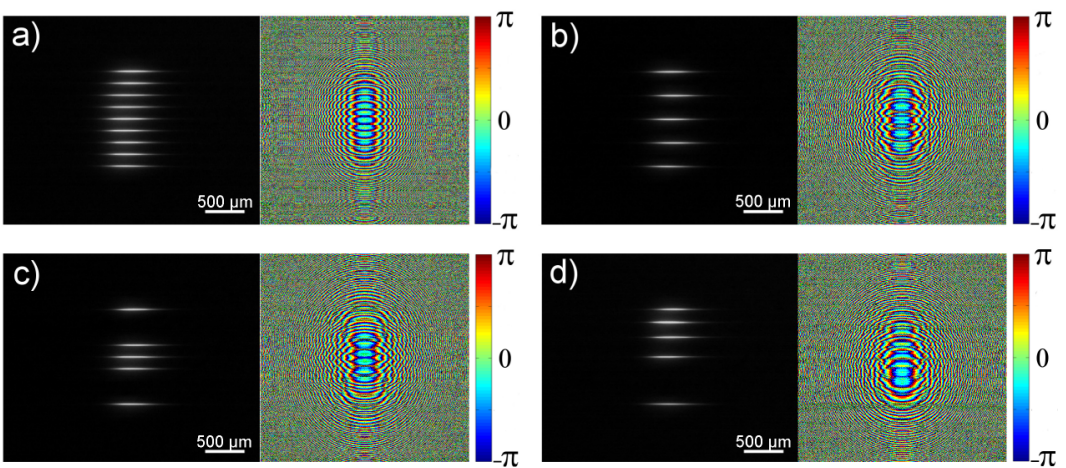

Fig. 3. Recorded images of different spatial distributions of filaments in fused silica due to the focusing of ultrashort pulses with the DPEs shown aside.

To construct the DPEs, we follow the encoding method described in section 2. In all cases, these DPEs are included at the right-part of the filament's images in Fig. 3. Specifically, a set of nine filaments with relative lateral separations of $128 \mu \mathrm{m}$ are shown in Fig. 3(a). By changing convergent even lenses by highly divergent ones in the previous DPE e.g., with focal length of $-500 \mathrm{~mm}$, another symmetric spatial distribution but this time composed of five filaments with separations of $256 \mu \mathrm{m}$ was given in Fig. 3(b). The energy associated with the divergent wave at the focal region can be neglected in comparison with the energy of filaments at this region. Note that, as the light is no longer focused when divergent lenses are 
used, we can remove the corresponding filaments within the fused silica. Furthermore, for all distances among filaments such that interaction effects can be neglected e.g., like the cases shown in Fig. 3, the encoding method ensures an independent control over the behavior of each filament. With the help of another DPE, a set of five filaments with non-equal separations is achieved in Fig. 3(c). Finally, the potential of the encoding method to generate arbitrary spatial distributions of filaments is experimentally demonstrated in Fig. 3(d). In this last case, a set of five filaments with non-linear increased/decreased distances among them has been obtained.

At this point, it should be noted that both the energy and axial positions of filaments shown in Fig. 3 are almost the same. Small discrepancies in the positions are less than $10 \mu \mathrm{m}$, which are in the order of the pixel size of our SLM, whereas energy variances are only about $8 \%$. To get this, when necessary, the focal lengths of kinoform diffractive lenses were slightly modified to correct for unwanted effects due to real experimental conditions. We found that small misalignments of the beam onto the SLM plane led to visible changes of the axial positions of filaments within the fused silica. The effect of misalignments can be more clearly seen in Fig. 4 where sets of nine and five filaments achieved without, Figs. 4(a) or 4(c), and with, Figs. 4(b) or 4(d), modification of the focal lengths are shown. For instance, when that focal length of all lenses was fixed to $245 \mathrm{~mm}$ we recorded the image given in Fig. 3(c). In contrast, after setting the more convenient focal lenses $f_{1}=245.6 \mathrm{~mm}, f_{2}=245.0 \mathrm{~mm}$, $f_{3}=246.0 \mathrm{~mm}, f_{4}=245.0 \mathrm{~mm}$ and $f_{5}=243.7 \mathrm{~mm}$ we got the filament distribution shown in Fig. 4(d). The variable $f_{i}$ refers to the focal length of the lens $i$ within the DPE.
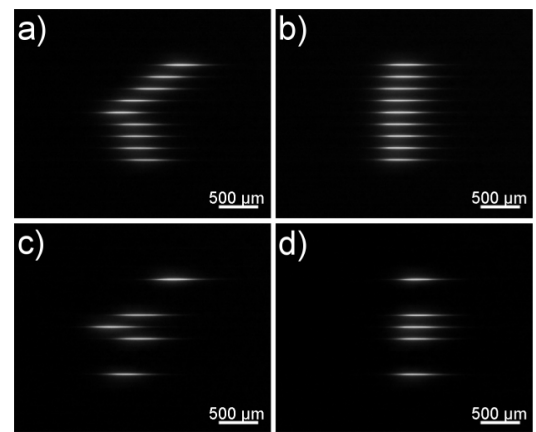

Fig. 4. Images of filaments in fused silica without (a, c) and with (b, d) corrections for the focal lengths.

On the other hand, if required, the focal energy coupled into the filaments can be conveniently decreased to finally obtain a similar amount of energy per filament. To do that, one can change the diffraction efficiency of each lens, modulating its quadratic phases $\Omega_{n}(x, y)$ with a multiplicative phase parameter $\delta$ that ranges from zero to one. In the above expression, the variables $x, y$ represent again transversal coordinates onto the DPE plane. This kind of shaping of the energy distribution per filament may be very useful in situations when the amplitude of the laser beam at the DPE plane evidences clear inhomogeneities. In this case, if a conventional array of lenses is used to focus the pulse like in [24], the amount of energy coupled into the filaments could be quite different. In contrast, distributions of filaments shown in Figs. 3 and 4 have almost the same intensity, so they seem to be not affected by this problem. For this reason, in this experiment corrections were only done in the focal lengths, but not in the diffraction efficiency of lenses. There are other well-known factors i.e., laser beam aberrations [21] or non-uniform spatial phase response [22-24] of the SLM, that also can change energies and positions of foci. However, in our experiment the high temporal stability of the obtained filaments suggests that possible effects introduced by 
time-dependent factors might be included into the scrambled distribution of filaments already shown in Figs. 4(a) and 4(c).

Here, it should be mentioned that under broadband spectral illumination each wavelength of the ultrashort pulse is focused by diffractive lenses at different axial positions. Specifically, these positions follow an inverse dependence with the wavelength of light. This happens, among other factors, because each diffractive lens can be optimized only for a particular wavelength (usually the central wavelength of the pulse) and a given focal length. So, one might expect that filaments generated by focusing ultrashort pulses with diffractive, instead of refractive lenses, show somehow a different behavior due to several reasons. For instance, for the same experimental conditions, i.e., equal numerical aperture, pulse energies and focal lengths, the Rayleigh range due to diffractive lenses is longer than the one obtained by focusing the pulse with a bulk refractive lens. In the temporal domain, basically owing to the propagation time difference among pulses coming from the center and edges of the diffractive lenses, the temporal duration of the pulse at the focus can significantly increase with respect to the temporal pulse width achieved with corresponding refractive lenses. The comparison of the filamentation process obtained with refractive and diffractive optics is beyond the scope of this manuscript, but a detailed analysis of this topic can be found elsewhere [25].

\section{Coherence properties of filaments}

In this section we experimentally demonstrate the usefulness of the encoding method to investigate the mutual coherence among filaments developed in fused silica. Note that, the possibility to bring filaments as closer as the pixel width of the SLM can lead to interference effects among them. In this experiment the ultrashort pulse is focused via DPEs into a fused silica to form an array of filaments which are able to interfere. The conical emission due to the nonlinear propagation of the pulse within the sample is superimposed to the interference patterns. The spectral broadening within the visible region of the electromagnetic spectrum originates colored interference patterns whose shapes will depend on the spatial distribution of filaments.

In contrast to a classical two arm interferometer which is usually highly dependent on environmental fluctuations and relatively difficult to align, the use of DPEs allows implementing a compact and robust optical system to measure the visibility of the interference fringes. It is basically composed of a couple of CCD cameras and a SLM. As explained in section 3, the CCD camera placed perpendicular to the propagation direction of the filaments is used to see the straight forward interference patterns, whereas the second camera placed aside the fused silica allows recording the weak plasma emissions or filaments. A complete schematic representation of the optical layout can be seen in Fig. 2. In this optical setup, a suited filter (model KG5, with $25 \mathrm{~mm}$ of diameter from Edmund Optics) is utilized to remove non-converted infrared light. For this application, each DPE can be regarded as a programmable single arm interferometer which is poorly dependent on certain unwanted phenomena i.e., mechanical vibrations. Although DPEs cannot be used to significantly vary the delay among pulses that develop in different filaments, the positions of them inside the fused silica can be precisely controlled (as demonstrated in section 3). In addition, the energy coupled into the filaments could be also modified by decreasing the above-mentioned phase parameter $\delta$ associated with the diffraction efficiency of the lenses. However, owing to the homogeneous spatial sampling of the lenses throughout the DPE, all generated filaments are almost of the same energy, so the need from diffraction efficiency compensation i.e., for instance claimed in [24], is not an issue in the present experiment.

In Fig. 5, the shape of interference patterns for different lateral separations/distances among filaments distributed according to two spatial configurations are shown. The selected distances 1 among filaments appear as an inset in the right-top part of corresponding images. In the left-bottom part of the images indications of their longitudinal scales are included. Typical longitudinal fringe patterns arise when two filaments interfere, whereas for an arbitrary 3D distribution of filaments more complex spot-like patterns come out. In particular, at the left-part of Fig. 5, two similar filaments located approximately at the same axial 
positions, but having increased/decreased lateral separations between them are shown. The corresponding interference pattern is shown aside. Similarly, at the right-part of Fig. 5, with the same lateral separations as before and following the 3D spatial distribution, images of three filaments and their interference patterns are shown. These three constituent filaments are located in the vertices of an equilateral triangle. In this case, note that visible variations in the intensity of filaments are mainly due to locations of filaments at different planes within the triangular distribution. As it might be expected, the longer the distance among filaments the shorter the period of the interference fringes will be, and vice versa. In addition, one can see that interference patterns are color-dependent. We found out that the distribution of colors within the interference patterns changes with the penetration depth of filaments within the fused silica. This effect seems to be related to two main factors, the change of chromatic aberrations at the focal regions achieved for different focal lengths, and the variation in the amount of material dispersion introduced by the fused silica for different penetration depths.

On the other hand, it is apparent that changes in the lateral distance among filaments should not alter the visibility of the interference patterns. In fact, from Fig. 5 one can roughly see that the contrast of these patterns is more or less the same in all cases. This guarantees a high coherence among the different filaments. However, this is not longer true when variations of distances take place in the axial instead of the lateral direction to the propagation direction of the filaments.
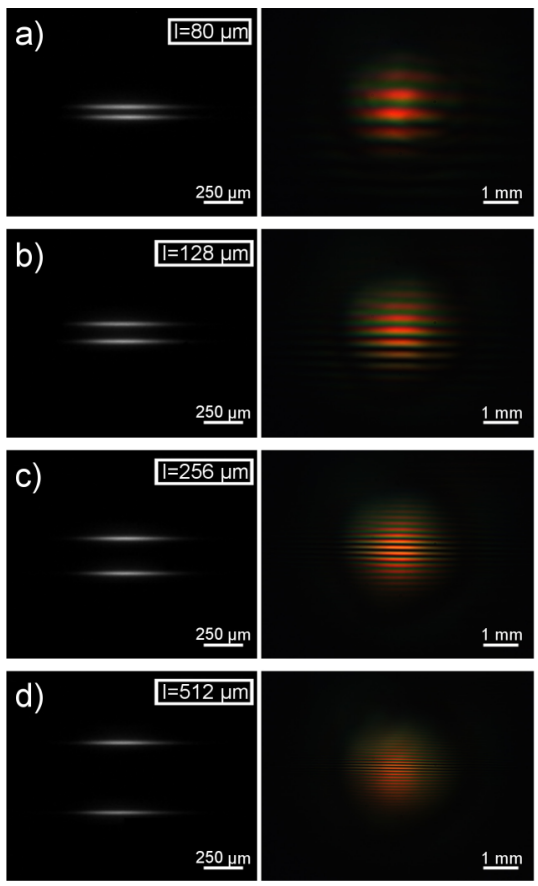
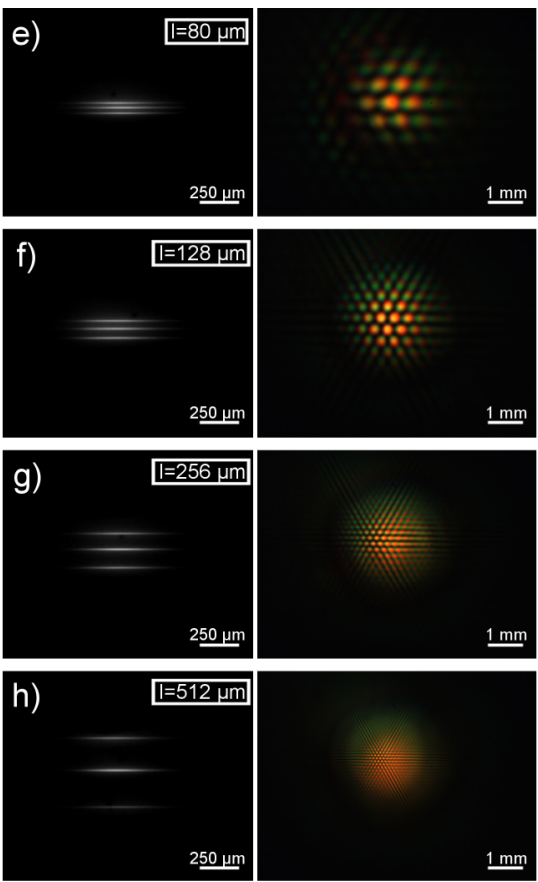

Fig. 5. Images of filaments with corresponding interference patterns for different lateral separations and spatial distributions. (a-d) two filaments located at the same plane (e-h) three filaments located in the vertexes of an equilateral triangle.

In the next experiment we want to show that using DPEs it is possible to investigate the effect of the axial separation of two filaments on the visibility of the interference pattern. Our experimental results are shown in Fig. 6. The axial distances d shown as insets in the righttop part of Figs. 6(a)-6(e) are taken with respect to the upper filament which is kept fixed. To change the distance between filaments, the focal length of the diffractive lens associated with the lower filament was ranged from $241.8 \mathrm{~mm}$ to $248.0 \mathrm{~mm}$ by means of variable increments.

As the interference patterns are color-dependent, different visibility values were calculated for each image corresponding to the red, green and blue (RGB) channels of the camera. These 
visibility values were determined for a couple of central fringes, using an irradiance profile taken at the middle of each interference pattern. These irradiance profiles (also plotted with red, green and blue colors) are shown in the right-part column of Fig. 6, together with the corresponding three values of visibility added as insets. For comparison, all visibility values were normalized with respect to the value achieved with the red channel in Fig. 6(c). This maximum value corresponds to the situation when there is no separation between upper and lower filaments. In this case, the visibility assessed due to the remaining channels is also the greatest. Therefore, the visibility worsens while increasing the axial separation between filaments. In these cases, the optical path and the delay between both filaments is no longer zero because original infrared pulses pass through different amounts of material dispersion.

The experimental results shown in Fig. 6 also reveal that apart from the apparent changes of visibility with the axial separation of filaments, there is an additional factor that could be taken into account.
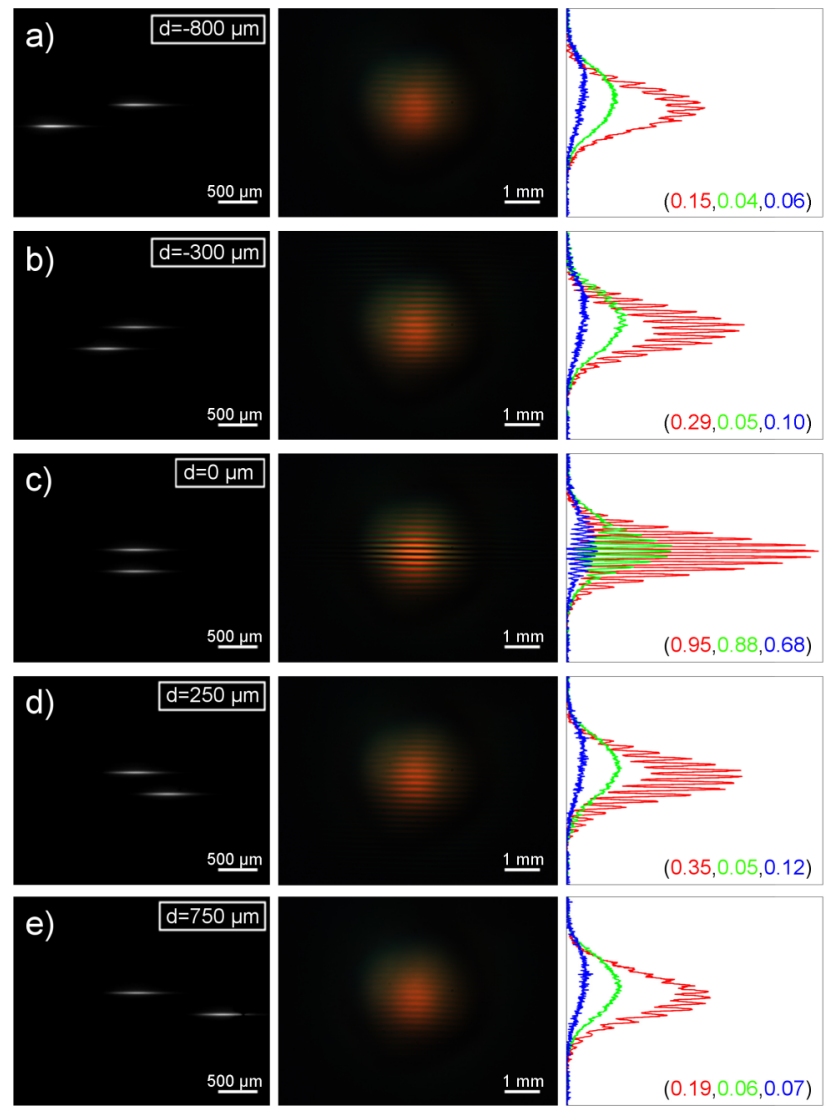

Fig. 6. Images of filaments (left-column) with corresponding interference patterns (middle column) together with their RGB central profiles (right-column) for different axial separations.

This factor is the different behavior of the visibility parameter for each RGB image of the interference pattern. For instance, for the experimental parameters used to get Fig. 6 i.e., penetration depths of filaments within the fused silica of about $4 \mathrm{~mm}$ or focal lengths around $245.0 \mathrm{~mm}$, the values of visibility achieved with the red channel are less affected by variations of the axial position of filaments than corresponding values due to the remaining channels. However, after changing the penetration depth of filaments within the fused silica, the behavior of visibility parameter for each RGB images also varies. We found that, among other reasons, this phenomenon is linked to the visible change in the azimuthal distribution of colors (conical emission) within the interference pattern due to the modification of the 
penetration depth. The decrease of the visibility with the axial displacement is related to the variations in the spatial and temporal overlapping conditions. Note that when filaments develop at different penetration depths within the material, the intensity distribution of the spectral components also changes, affecting the interference process.

In order to show this effect, a set of interference patterns obtained for three different penetration depths (included with the variable $\mathrm{p}$ in their top-right parts) are shown in Fig. 7. In this experiment, the interference patterns were originated by the interaction of a couple of filaments having lateral $(1=216 \mu \mathrm{m})$, but not axial $(\mathrm{d}=0)$ separations between them.
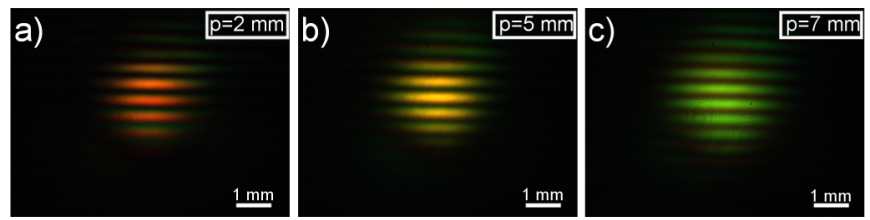

Fig. 7. Interference patterns due to the interaction of a couple of filaments for different penetration depths within the fused silica.

To approximately introduce the same amount of chromatic aberrations, the focal length ( $f=280 \mathrm{~mm}$ ) of the two diffractive lenses employed to generate the filaments remains the same during the whole experiment. Instead, with the help of a motorized stage, the fused silica was moved with respect to the filaments. After a visual inspection of Fig. 7, one can conclude that the predominant color within the interference patterns is shifted when modifying the penetration depth. In this context, several effects on the spatial, spectral, and temporal characteristics of filaments for different positions of a fused silica with respect to a fabricated kinoform DL (Institute of Automatics and Electrometry, Russia) have been recently reported [26]. In particular, the tunability of the spectrum in the anti-Stokes side was experimentally demonstrated, see Fig. 2 in [26]. So, the spectral behavior shown in Fig. 7 should depends on several factors, including material dispersion, initial pulse chirp, or extension of the focal length. For instance, in the time domain increasing/decreasing the material dispersion implies variations in the temporal width of the pulse, which clearly influence the development of filaments within the fused silica, and consequently the characteristics of corresponding interference patterns.

\section{Conclusions}

In this manuscript, we experimentally showed that DPEs encoded into a phase-only SLM can be successfully utilized to generate arbitrary 3D spatial distributions of filaments in bulk optics with micrometric spatial resolution. The spatial sampling procedure employed to construct each DPE allows having high accuracy and independent control over some physical parameters of filaments such as the energy coupled into the filaments or their positions within the fused silica. We found that both the coupled energy and the relative positions of filaments can be conveniently tuned by changing the diffraction efficiency, and the center of the corresponding off-axis kinoform diffractive lenses, respectively. The usefulness and robustness of DPEs for practical applications were tested with a couple of experiments addressed to study the mutal coherence of filaments while they develop inside the fused silica. In particular, we showed that visibility of interference patterns due to the interaction of filaments within a predefined spatial distribution changes when modifying the relative axial distances among filaments. Its maximum value is achieved when there is no axial separation between filaments. In addition, for a selected pair of fringes we found that the visibility also depends on the RGB image associated with the interference pattern.

In comparison with previous studies aimed to the control of multifilamentation processes in fused silica by using arrays of diffractive lenses, our proposal showed some advances. The most significant one is the possibility to bring filaments as close as the pixel width of the SLM without using additional optical components. Another advance is the tested ability of 
DPEs to generate filaments with relative similar energies, regardless the homogeneity of the beam's irradiance onto the DPE. These advances are made possible mainly due to the homogeneous spatial sampling of the diffractive lenses, which also guarantees high numerical aperture for the mix of lenses. However, with the encoding method used in this manuscript one can encode only a limited amount of lenses, basically because the sampling of the phase associated with each lens gets worse when increasing the number of lenses in the DPE. This drawback might be softened if technology behind the next generation of SLMs allows for devices with better resolution and lower pixel width.

\section{Acknowledgments}

We acknowledge support from Generalitat Valenciana through the programme PROMETEO2012-021, University Jaume I through the project P1·1B2013-53, and Ministerio de Economía y Competitividad (MINECO) through the project FIS2013-40666-P. The authors are also very grateful to the SCIC of the Universitat Jaume I for the use of the femtosecond laser. 


\title{
Video Article \\ Shaping the Amplitude and Phase of Laser Beams by Using a Phase-only Spatial Light Modulator
}

\author{
Miguel Carbonell-Leal ${ }^{1}$, Omel Mendoza-Yero ${ }^{1}$ \\ ${ }^{1}$ Institut de Noves Tecnologies de la Imatge (INIT), Universitat Jaume I \\ Correspondence to: Omel Mendoza-Yero at omendoza@fca.uji.es \\ URL: https://www.jove.com/video/59158 \\ DOI: doi: $10.3791 / 59158$
}

Keywords: Engineering, Issue 143, Phase modulation, encoding complex field, spatial light modulator, common-path interferometer

Date Published: 1/28/2019

Citation: Carbonell-Leal, M., Mendoza-Yero, O. Shaping the Amplitude and Phase of Laser Beams by Using a Phase-only Spatial Light Modulator. J. Vis. Exp. (143), e59158, doi:10.3791/59158 (2019).

\section{Abstract}

The aim of this article is to visually demonstrate the utilization of an interferometric method for encoding complex fields associated with coherent laser radiation. The method is based on the coherent sum of two uniform waves, previously encoded into a phase-only spatial light modulator (SLM) by spatial multiplexing of their phases. Here, the interference process is carried out by spatial filtering of light frequencies at the Fourier plane of certain imaging system. The correct implementation of this method allows arbitrary phase and amplitude information to be retrieved at the output of the optical system.

It is an on-axis, rather than off-axis encoding technique, with a direct processing algorithm (not an iterative loop), and free from coherent noise (speckle). The complex field can be exactly retrieved at the output of the optical system, except for some loss of resolution due to the frequency filtering process. The main limitation of the method might come from the inability to operate at frequency rates higher than the refresh rate of the SLM. Applications include, but are not limited to, linear and non-linear microscopy, beam shaping, or laser micro-processing of material surfaces.

\section{Video Link}

The video component of this article can be found at https://www.jove.com/video/59158/

\section{Introduction}

Almost all laser applications are in close relation with the management of the optical wavefront of light. In the paraxial approximation, the complex field associated with the laser radiation can be described by two terms, the amplitude and the phase. Having control over these two terms is necessary to modify both the temporal and the spatial structure of laser beams at will. In general, the amplitude and the phase of a laser beam can be properly changed by several methods including the use of optical components that range from single bulk lenses, beam splitters and mirrors to most complex devices like deformable mirrors or spatial light modulators. Here, we show a method for encoding and reconstructing the complex field of coherent laser beams, which is based on dual-phase hologram theory ${ }^{1}$, and the utilization of a common-path interferometer.

Nowadays, there exists a wide variety of methods to encode the complex fields of laser beams $\mathrm{s}^{2,3,4,5}$. In this context, some well-established methods to produce phase and amplitude modulation rely on the use of digital holograms ${ }^{6}$. A common point in all these methods is the necessity of generating a spatial offset to separate the desired output beam from the zeroth-order coming from the reflection of light at the SLM display. These methods are basically off-axis (usually applying for the first diffraction order of the grating), employing phase grating not only to encode the phase, but also to introduce necessary amplitude modulation. In particular, amplitude modulation is performed by spatially lowering the grating height, which clearly degrades the diffraction efficiency. The hologram reconstruction process mostly gets an approximate, but not exact, reconstruction of the amplitude and phase of the desired complex field. Discrepancies between theory and experiment seem to appear from an inaccurate encoding of the amplitude information as well as other experimental issues happening during the spatial filtering of the first diffraction order or due to SLM pixilation effects. In addition, the intensity profile of the input beam can introduce restrictions on the output power.

In contrast, with the introduced method ${ }^{7}$, all light management is carried out on-axis, which is very convenient from an experimental point of view. Additionally, it takes advantage of considering, in the paraxial approximation, the complex field associated with laser beams as a sum of two uniform waves. The amplitude information is synthetized by the interference of these uniform waves. In practice, such interference is carried out by spatial filtering of light frequencies at the Fourier plane of a given imaging system. Previously, the phase patterns associated with the uniform waves are spatially multiplexed and encoded into a phase-only SLM (placed at the entrance plane of this imaging system). Hence, the whole optical setup can be regarded as a common-path interferometer (very robust against mechanical vibrations, temperature changes, or optical misalignments). Please, note that the abovementioned interference process can be alternatively accomplished by using other optical layouts: with a couple of phase-only SLMs properly placed within a typical two-arm interferometer, or by time sequentially encoding the two phase patterns into the SLM (previous introduction of a reference mirror in the optical setup). In both cases, there is no necessity of spatial filtering, and consequently no loss of spatial resolution, at the expense of increasing the complexity of the optical system, as well as the alignment process. 
Here, it should be also emphasized that by using this encoding method, the full spectrum of the desired complex field can be exactly retrieved at the Fourier plane, after filtering all diffraction orders but the zeroth one.

On the other hand, the efficiency of the method depends on several factors: the manufacturer's specifications of the SLM (e.g., fill factor, reflectivity, or diffraction efficiency), the size of the encoded pattern, and the way at which the light impinges onto the SLM (reflection with a small hitting angle, or normal incidence by using a beam splitter). At this point, under proper experimental conditions, the measured total light efficiency can be more than $30 \%$. However, note that that the total light efficiency just due to the use of the SLM can be less than $50 \%$. The lack of random or diffuser elements within the optical setup allows the retrieving of amplitude and phase patterns without coherent noise (speckle). Other significant aspects to point out are the utilization of a direct codification algorithm rather than iterative procedures and its ability to perform arbitrary and independent amplitude and phase modulation at the frequency refresh time of the SLM (up to hundreds of hertz according to the current technology).

In principle, the method ${ }^{7}$ is intended to be used with input plane waves, but it is not limited to that. For instance, if a Gaussian beam is hitting the SLM, it is possible to modify its irradiance shape at the output of the system by encoding a suited amplitude pattern into the SLM. However, as the intensity of the output beam cannot exceed that of the input beam at any transversal position (x,y), the shaping of the amplitude is performed by intensity losses originated by a partially destructive interference process.

The theory underlining the encoding method ${ }^{7}$ is as follows. Any complex field represented in the form $U(x, y)=A(x, y) e^{i \varphi(x, y)}$ can be also rewritten as:

$U(x, y)=B e^{i \theta(x, y)}+B e^{i \vartheta(x, y)}$

where

$\theta(x, y)=\varphi(x, y)+\cos ^{-1}\left[A(x, y) / A_{\max }\right](2)$

$\vartheta(x, y)=\varphi(x, y)-\cos ^{-1}\left[A(x, y) / A_{\max }\right](3)$

In equations 1-3, the amplitude and phase of the two-dimensional complex field $U(x, y)$ is given by $A(x, y)$ and $\varphi(x, y)$, respectively. Note that, terms $A_{\max }$ (maximum of $A(x, y)$ ) and $B=A_{\max } / 2$ do not depend on the transversal coordinates $(x, y)$. From the theory, if we set $A_{\max }=2$, then $B=1$. Hence, the complex field $U(x, y)$ can be obtained, in a simple manner, from the coherent sum of uniform waves $B e^{i \vartheta(x, y)}$ and $B e^{i \theta(x, y)}$. In practice, this is accomplished with a common-path interferometer made up of a single phase element $\alpha(x, y)$, placed at the input plane of an imaging system. The single phase element is constructed by spatial multiplexing of the phase terms $\vartheta(x, y)$

and $\theta(x, y)$ with the help of two-dimensional binary gratings (checkerboard patterns) $M_{1}(x, y)$ and $M_{2}(x, y)$ as follows

$M_{1}(x, y) e^{i \theta(x, y)}+M_{2}(x, y) e^{i \vartheta(x, y)}=e^{i \alpha(x, y)}(4)$

hence,

$\alpha(x, y)=M_{1}(x, y) \theta(x, y)+M_{2}(x, y) \vartheta(x, y)(5)$

These binary patterns fulfill the condition $M_{1}(x, y)+M_{2}(x, y)=1$. Note that, the interference of uniform waves cannot happen if we do not mix the information contained in the phase element $\alpha(x, y)$. In the present method, this is carried out by using a spatial filter able to block all diffraction orders but the zeroth one. In this way, after the filtering process at the Fourier plane, the spectrum $H(u, v)=F\left\{e^{i \alpha(x, y)}\right\}$ of the encoded phase function is related to the spectrum of the complex field $F\{U(x, y)\}$ by the expression

$H(u, v) P(u, v)=\frac{F\{U(x, y)\}}{2}(6)$

In Eq. (6), $(u, v)$ denote coordinates in the frequency domain, $P(u, v)$ holds for the spatial filter, whereas the Fourier transform of a given function $\Theta(x, y)$ is represented in the form $F\{\Theta(x, y)\}$. From Eq. (6), it follows that, at the output plane of the imaging system, the retrieved complex field $U_{R E T}(x, y)$, (without considering constant factors), is given by the convolution of the magnified and spatially reversed complex field $U(x, y)$ with the Fourier transform of the filter mask. That is:

$U_{R E T}(x, y)=U(-x / \operatorname{Mag},-y / \operatorname{Mag}) \otimes F\{P(u, v)\}(7)$

In Eq. (7), the convolution operation is denoted by the symbol $\otimes$, and the term Mag represents the magnification of the imaging system. Hence, the amplitude and phase of $U(x, y)$ is fully retrieved at the output plane, except for some loss of spatial resolution due to the convolution operation.

\section{Protocol}

\section{Encoding the Complex Field into a Single Phase Element}

1. From the technical specifications of the SLM, find its spatial resolution (for instance 1920 pixels $\times 1800$ pixels).

2. Define and generate the desired amplitude $A(x, y)$ and phase $\varphi(x, y)$ patterns as digital images.

1. Set the spatial resolution of abovementioned digital images equal to that of the SLM display. 
2. Set abovementioned digital images in gray level format.

3. Set the minimum and maximum values of the amplitude and phase images from 0 to 255 , and from $-\pi / 2$ to $\pi / 2$, respectively.

4. Set $A_{\max }=2$ in equations 2 and 3 , and computer-generate the phase patterns $\vartheta(x, y)$ and $\theta(x, y)$ from them.

3. Computer generate the checkerboard patterns $M_{1}(x, y)$ and $M_{2}(x, y)$.

1. Set the spatial resolution of these checkerboard patterns equal to that of the SLM display.

2. To reduce the effect of pixel crosstalk, generate other pairs of checkerboard patterns $M_{1}(x, y)$ and $M_{2}(x, y)$ constructed with different pixel cells having an increased number of pixels (for instance: $2 \times 2,3 \times 3$, and $4 \times 4$ pixel cells, etc.).

CAUTION: When increasing the pixel cell, the total number of pixels of checkerboard patterns must be kept unchanged and equal to the spatial resolution of the SLM. Ensure that final number of pixels of all checkerboard patterns remains the same after modifying their pixel cells.

4. Computer generate the single phase element $\alpha(x, y)$ from equation 5 .

NOTE: See supplemental material named "MATLAB_code_1.m" for related tasks on step 1 of this protocol.

\section{Reconstruction of the Complex Field}

1. Use a collimated, linear polarized, and spatially coherent laser beam as a light source.

2. Use a phase-only SLM with at least $2 \pi$ phase range.

3. When necessary, use a proper beam expander to adjust the size of the beam to the size of the SLM display.

4. When necessary, use an optical polarizer to set laser beam polarization to the horizontal direction. This is usually important for the proper operation of phase-only SLMs, which are typically designed to modulate the spatial phase of the electromagnetic field that oscillate in the horizontal direction, keeping unchanged its vertical components.

5. In order to send a phase pattern to the SLM, follow standard communication protocols given by the SLM's manufacturer to connect and control the SLM with the computer.

NOTE: Common protocol for this purpose includes the use of a calibration curve to transform the values in radians (due to mathematical operations with angles) into gray level ones, which the electronic control unit of the SLM will finally convert into voltage levels. Additionally, as the SLM is connected to computer as an external device with its own screen, an extension of the computer screen is usually necessary, as well as a proper program to send the corresponding gray level images to this extra screen. An example of these codes is also included as supplemental material (please, see MATLAB_code_2.m).

6. Implement an image optical system and put the display of the SLM in the input plane of this system.

1. Use a refractive lens of a focal length $f$ to construct a $2 f \times 2 f$ optical image system (a $4 f$ optical system is also valid for this task). In accordance with the expected output size of the complex field, beam width, wavelength of light, and the available physical space, employ lens/lenses with suited technical specifications (e.g., coating, size, focal length, etc.).

2. To find the position of the output plane of the imaging system, send the phase pattern $\alpha(x, y)$ to the SLM and visually look for the recorded image (depending on the position of the camera) with the best spatial resolution.

CAUTION: In the case of low-size pixel cells (for instance, $1 \times 1$ pixel cells) and SLM displays with pixel widths of a few microns (for instance, $8 \mu \mathrm{m}$ ), only beam propagation can produce interference between encoded uniform waves, getting a reconstructed images without including the circular iris in the imaging system. Use low-size pixel cells to locate the position of the output plane.

3. Place a circular iris of variable diameter at the Fourier plane of the optical system and align its center with that of the laser beam focus.

4. To adjust the size of the circular iris at the Fourier plane, send the phase pattern $\alpha(x, y)$ and visually look for the recorded image (depending on the diameter of the circular iris) with best spatial resolution.

CAUTION: In the case of long-size pixel cells (for instance, $4 \times 4$ pixel cells), the interference between encoded uniform waves is basically carried out with the spatial filter. Use long-size pixel cell to adjust the size of the circular iris. In this protocol, the terms low-size and long-size are referred to the number of pixels contained within a pixel cell. However, the abovementioned interference depends also on the pixel width. Employ SLMs with pixel widths equal or less than $8 \mu \mathrm{m}$.

7. Send the gray level image corresponding to the phase element $\alpha(x, y)$ to the SLM.

1. To minimize the crosstalk effect, look for the best pixel cell size which allow achieving the recorded image with the higher spatial resolution.

\section{Measure the Reconstructed Complex Field}

1. Implement the polarization-based phase shifting technique ${ }^{8}$.

1. Place and align the rotation angle of the first optical polarizer, located just before the SLM (see Figure 2). To set the rotation angle of the first polarizer, visually look for the maximum and minimal light transmittance in the CCD camera (placed at the output plane of the imaging system), depending on the rotation of the polarizer. Write down the two corresponding angles of the polarizer. Fix the final angle of the polarizer to that between the two previous-recorded angles.

2. Place and align the rotation angle of the second optical polarizer, located after the Fourier plane of the imaging system (see Figure 2). To set the rotation angle of the second polarizer, visually look for the sharpest and most blurred images in the CCD camera (placed at the output plane of the imaging system) after sending the phase pattern $\alpha(x, y)$ to the SLM. Write down the two corresponding angles of the polarizer. Fix the final angle of the second polarizer to that between the previous-recorded angles.

2. Record the interferograms.

1. Keep the CCD camera at the output plane of the imaging system.

2. To record the first interferogram, add a matrix of 0 radians to the phase element $\alpha(x, y)$ and send it to the SLM. Record corresponding image $I_{1}(x, y)$ with the CCD. 
3. To record the second interferogram, add a matrix of $\pi / 2$ radians to the phase element $\alpha(x, y)$ and send it to the SLM. Record corresponding image $I_{2}(x, y)$ with the CCD camera.

4. To record the third interferogram, add a matrix of $\pi$ radians to the phase element $\alpha(x, y)$ and send it to the SLM. Record corresponding image $I_{3}(x, y)$ with the CCD camera.

5. To record the fourth and last interferogram, add a matrix of $3 \pi / 2$ radians to the phase element $\alpha(x, y)$ and send it to the SLM. Record corresponding image $I_{4}(x, y)$ with the CCD camera.

3. Reconstruct the complex field.

NOTE: See supplemental material named "MATLAB code 3.m" for related tasks on this point of the protocol.

1. Retrieve the amplitude of the complex field $A_{\text {retrieved }}(x, y)$ by using the expression

$$
A_{\text {retrieved }}(x, y)=\sqrt{\left[I_{3}(x, y)-I_{1}(x, y)\right]^{2}+\left[I_{4}(x, y)-I_{2}(x, y)\right]^{2}}(8)
$$

2. Retrieve the phase of the complex field $\varphi_{\text {retrieved }}(x, y)$ by using the expression

$$
\varphi_{\text {retrieved }}(x, y)=\arctan \left[\frac{I_{4}(x, y)-I_{2}(x, y)}{I_{3}(x, y)-I_{1}(x, y)}\right](9)
$$

\section{Representative Results}

The spatial resolution of the employed phase-only SLM is 1920 pixels $x 1080$ pixels, with a pixel pitch of $8 \mu$ m. The selected amplitude $A(x, y)$ and phase $\varphi(x, y)$ of the complex field are defined by two different gray level images corresponding to the well-known Lenna's picture (amplitude pattern) and a young girl sticking out her tongue (phase pattern), respectively. In general, for both, the generation of necessary patterns, and the control of the SLM, Matlab codes are utilized. The spatial resolution of these images is set to be 1920 pixels $\times 1080$ pixels. Then, equations 2 and 3 are used to determine the phase patterns $\vartheta(x, y)$ and $\theta(x, y)$ for $A_{\max }=2$. Note that, the numerical value given to $A_{\max }$ guaranties that term $B=1$ and consequently, the complex field $U(x, y)$ described by Eq. (1) can be understood as the sum of two uniform waves in the simplest form $U(x, y)=e^{i \vartheta(x, y)}+e^{i \theta(x, y)}$. Now, different pairs of binary checkerboard patterns $M_{1}(x, y)$ and $M_{2}(x, y)$ (for increased pixel cell sizes), but equal spatial resolution (1920 pixels $\times 1080$ pixels), are computer generated. Particularly, checkerboard patterns made up of $1 \times 1,2 \times 2,3 \times 3$ and $4 \times 4$ pixel cells are digitally constructed by using a programed Matlab function. All abovementioned patterns $A(x, y), \varphi(x, y), \vartheta(x, y), \theta(x, y), M_{1}(x, y)$, and $M_{2}(x, y)$ are shown in parts $A, B, C, D, E$, and F of Figure 1, respectively. In parts $E$ and $F$, and just to get a better visualization of the structure of checkerboard patterns, the constituent pixel cells are of 240 pixels $\times 240$ pixels. From Eq. 5, a set of phase elements $\alpha(x, y)$ for each pair of previously-designed checkerboard patterns are digitally constructed.

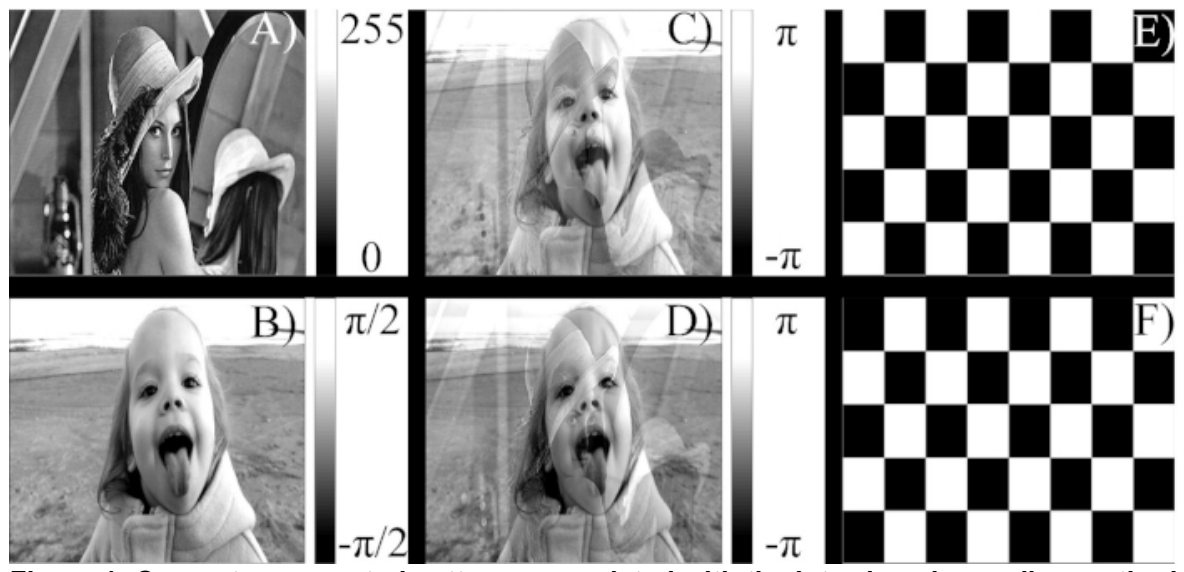

Figure 1: Computer generated patterns associated with the introduced encoding method. (A) User-defined amplitude pattern of the complex field. (B) User-defined phase pattern of the complex field. (C) Phase pattern corresponding to the first uniform wave in equation 1. (D) Phase pattern corresponding to the second uniform wave in equation 1. (E) First checkerboard pattern following the sampling process described with equation 4. (F) Second checkerboard pattern following the sampling process described with equation 4 . Please click here to view a larger version of this figure.

At this point, the expected complex field $U(x, y)$ can be experimentally retrieved at the output plane of an imaging system, once the phase element $\alpha(x, y)$ is sent to the phase-only SLM and the interference between encoded uniform waves takes place. To perform this interference, a spatial filter (for instance, a circular iris) is adjusted in size to block all frequencies but the zeroth one at the Fourier plane of the imaging system (Figure 2). 


\section{Laser beam}

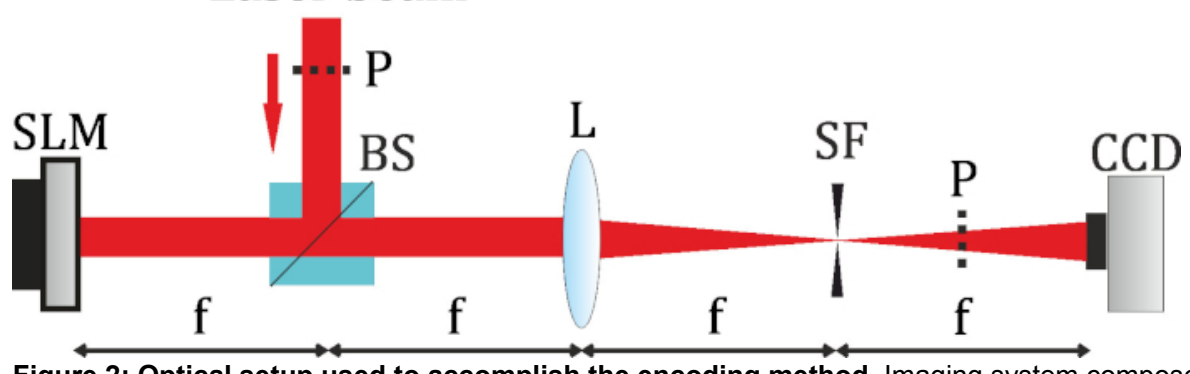

Figure 2: Optical setup used to accomplish the encoding method. Imaging system composed of a spatial light modulator (SLM), beam splitter (BS), and single refractive lens (L) of focal length $200 \mathrm{~mm}$. In the Fourier plane is included a hard iris, which is employed as a spatial filter (SF) to block all frequencies but the zero one. In addition, at the output plane of the imaging system is placed a camera (CCD) to record amplitude patterns, and interferograms. Only to measure the generated complex field by means of polarization-based phase shifting technique, a couple of optical polarizers $(P)$ are properly located within the optical setup. Please click here to view a larger version of this figure.

As a light source, a Ti: Sapphire laser oscillator (working out of the mode locked condition to emit a quasi-monochromatic laser radiation of about $10 \mathrm{~nm}$ intensity full width at half maximum (FWHM) and centered at $800 \mathrm{~nm}$ ) is employed. In addition, to fill almost all the active area of the SLM display $(8.64 \mathrm{~cm} \times 15.36 \mathrm{~cm}$ ) with the laser beam, a commercial $5 \times$ telescope beam expander is used. The laser beam is sent (in normal incident) to the display of the SLM by means of a pellicle beam splitter. A refractive lens of focal length $100 \mathrm{~mm}$ is placed $200 \mathrm{~mm}$ after the SLM and aligned with respect to the optical axis of the laser beam reflected back from the SLM. To locate the position of the output plane of the imaging system, the image of $A(x, y)$ recorded the CCD camera was found. This is done once the phase element $\alpha(x, y)$ (formed with $1 \times 1$ pixel cells) is sent to the SLM. Then, a circular iris is placed at the Fourier plane of the optical system, and aligned with respect to the focus of the laser beam. In addition, to adjust the size of the circular iris, its diameter is varied until better image reconstruction is achieved by visual inspection in the CCD camera. To this purpose, the phase element $\alpha(x, y)$ (digitally constructed with $4 \times 4$ pixel cells) was previously sent to the SLM. To minimize the effect of pixel crosstalk, the best phase element $\alpha(x, y)$ (depending on the pixel cell size) that allows achieving the image with higher spatial resolution in the CCD is found.

In order to corroborate that the desired complex field is reconstructed at the output plane of the imaging system, the already-mentioned polarization-based phase shifting technique is used to measure its amplitude and phase. To do that, a couple of polarizers $p$ (one placed before the SLM, and another after the output plane of the imaging system) are properly aligned within the optical setup (see Figure 2), following the procedure described in steps 3.1.1 and 3.1.2 of the protocol. Then, the interferograms associated with the four-step phase shifting technique $I_{1}(x, y), I_{2}(x, y), I_{3}(x, y)$, and $I_{4}(x, y)$ are recorded with the CCD camera (already placed at the output plane of the imaging system). Here, it should be recalled that these four interferograms are recorded with the camera after the addition of $0, \pi / 2$, $\pi$, and $3 \pi / 2$ to the phase element $\alpha(x, y)$ (see steps 3.2.2 - 3.2.5 of the protocol for details). Finally, using equations 8 and 9, the amplitude and phase of the reconstructed complex field can be retrieved. For this experiment, the results are shown in Figure 3.

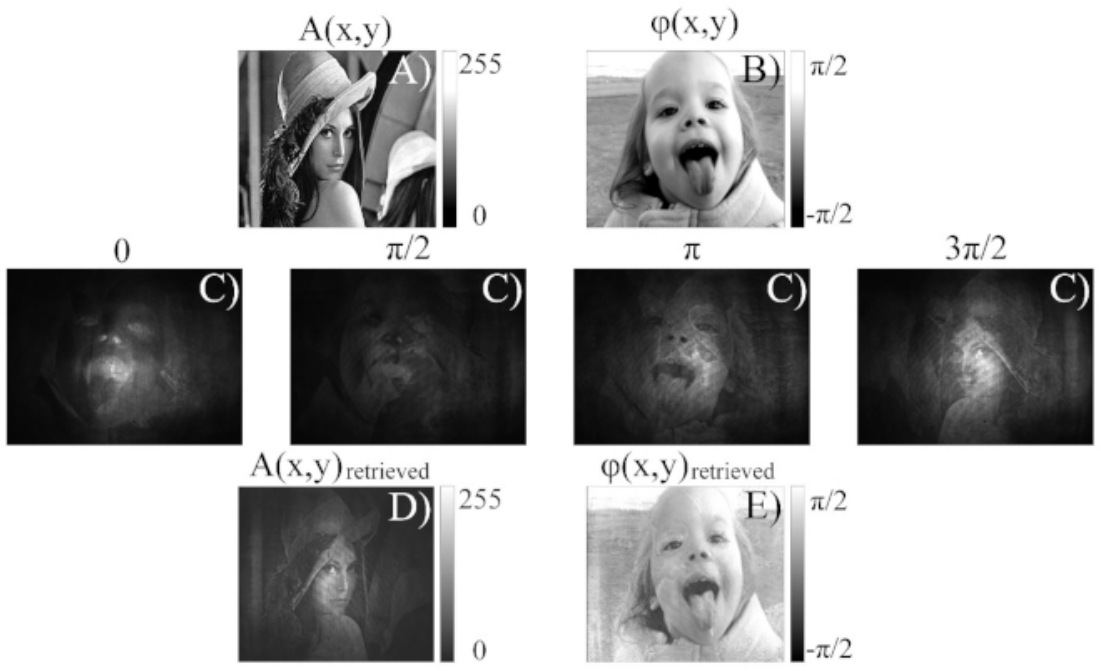

Figure 3: Representative experimental results under quasi-monochromatic illumination. (A) User-defined amplitude pattern of the complex field. (B) User-defined phase pattern of the complex field. (C) Interferograms associated with the polarization-based phase shifting technique developed in four steps and obtained after adding $0, \pi / 2, \pi$, and $3 \pi / 2$ to the phase element $\alpha(x, y)$. (D) Retrieved experimental amplitude pattern. (E) Retrieved experimental phase pattern. Please click here to view a larger version of this figure.

Supplemental Materials. Please click here to download the files. 


\section{Discussion}

In this protocol, practical parameters as the pixel width of the phase-only SLM or the number of pixels contained within pixel cells of a computergenerated pattern are key points to successfully implement the encoding method. In steps 1.2, 1.3, and 1.4 of the protocol, the shorter the pixel width, the better the spatial resolution of the retrieved amplitude and phase patterns. In addition, as the codification into the SLM of abrupt pixelto-pixel phase modulations can originate unexpected phase responses (pixel crosstalk), the construction of checkerboard patterns (as described in step 1.3) should be linked to the increment of the number of pixels within pixel cells. The main reason to do that is to mitigate the effects of pixel crosstalk on the retrieved amplitude and phase patterns. However, when increasing the number of pixels within the pixel cells, the spatial resolution of the recorded complex field patterns $A_{\text {retrieved }}(x, y)$ and $\varphi_{\text {retrieved }}(x, y)$ is decreased. Hence, having high spatial resolution SLMs with low pixel widths allows reducing possible crosstalk effects, without losing significant spatial resolution in the retrieved amplitude and phase patterns.

Furthermore, in step 1.2.3 of the protocol, the phase of the complex field is defined from $-\pi / 2$ to $\pi / 2$. The main reason for setting such phase range is to generate a phase element $\alpha(x, y)$ ranging from $-\pi$ to $\pi$, which can be implemented into a SLM with $2 \pi$ of phase range. However, if the phase range of the available SLM is greater than $2 \pi$, the phase of the complex field could be defined within an extended range (for instance: for $\varphi(x, y)$ ranging from $-\pi$ to $\pi$, the phase element $\alpha(x, y)$ may range from $-3 \pi / 2$ to $3 \pi / 2$, and consequently the phase range of the SLM must be, at least, $3 \pi)$.

The characteristics of the laser beam could also influence the results of the encoding method. Pay special attention to the steps 2.1-2.4, setting the right polarization direction, collimation, and transversal size of the laser beam before following the remaining steps of the protocol. Furthermore, as phase-only SLMs are basically diffractive-dependent optical devices based on the interference phenomenon, it is necessary to use laser beams with high/good spatial coherence.

On the other hand, instead of quasi-monochromatic, ultrashort pulsed illumination also allows obtaining good results. In this case, the different spectral components of the pulse are phase modulated (in a very similar manner) just with the single phase element $\alpha(x, y)$. Here, to show the effect of a broadband light source on the encoding method, we repeat all steps of the protocol, but this time for pulsed radiation (an ultrashort pulse of about $12 \mathrm{fs} \mathrm{FWHM,} \mathrm{centered} \mathrm{at} 800 \mathrm{~nm}$, spectral bandwidth of $100 \mathrm{~nm}$ FWHM, emitted by a mode-locked Ti:Sapphire laser from the femtolaser, at a $75 \mathrm{MHz}$ repetition rate). The results are shown in Figure 4. Note that, due to the mix of the different spectral components of the pulse, the retrieved patterns are very close to the expected ones.

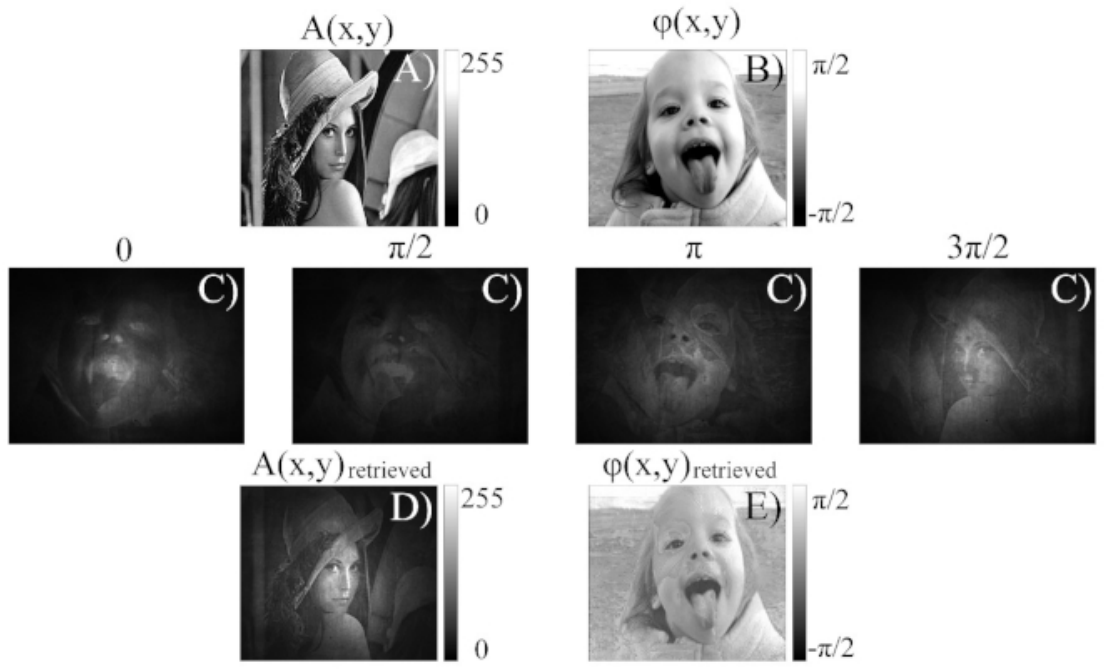

Figure 4: Representative experimental results under ultrashort pulsed illumination. (A) User-defined amplitude pattern of the complex field. (B) User-defined phase pattern of the complex field. (C) Interferograms associated with the polarization-based phase shifting technique developed in four steps and obtained after adding $0, \pi / 2, \pi$, and $3 \pi / 2$ to the phase element $\alpha(x, y)$. (D) Retrieved experimental amplitude pattern. (E) Retrieved experimental phase pattern. Please click here to view a larger version of this figure.

Laser beams are intrinsically complex fields, so in most potential applications one should be able to modify their amplitude and phase, simultaneously. The present method allows to do that by means of a single phase element (implemented or not into a phase-only SLM). We believe that, in a near future, this method could be employed, for instance in the illumination path of microscopes ${ }^{9,10}$ for simultaneous linear and non-linear excitation of different zones of biological samples, or in parallel micro-processing ${ }^{11,12}$ of materials. In both applications the role of amplitude modulation is apparent, meanwhile phase modulation can be utilized, at the same time, for compensation of optical aberrations at the sample/processing plane. Finally, it should be mentioned that the encoding method described with the present protocol is not limited to the utilization of SLMs. Fixed phase elements $\alpha(x, y)$ constructed with other techniques (for instance: photolithographic techniques) can be a different, but equally valid option to implement this protocol.

\section{Disclosures}

The authors have nothing to disclose. 


\section{Acknowledgments}

This research was supported by Generalitat Valenciana (PROMETEO 2016-079), Universitat Jaume I (UJI) (UJIB2016-19); and Ministerio de Economía y Competitividad (MINECO) (FIS2016-75618-R). The authors are very grateful to the SCIC of the Universitat Jaume I for the use of the femtosecond laser.

\section{References}

1. Hsueh, C. K., and Sawchuk, A. A. Computer-generated double-phase holograms. Applied Optics. 17 (24), $3874-3883$ (1978).

2. Arrizón, V. Complex modulation with a twisted-nematic liquid-crystal spatial light modulator: double-pixel approach. Optics Letters. 28 (15), 1359-1361 (2003).

3. Arrizón, V., Ruiz, U., Carrada, R., González, L. A. Pixelated phase computer holograms for the accurate encoding of scalar complex fields. Journal of the Optical Society of America A. 24 (11), 3500-2507 (2007).

4. Shibukawa, A., Okamoto, A., Takabayashi, M., Tomita, A. Spatial cross modulation method using a random diffuser and phase-only spatial light modulator for constructing arbitrary complex fields. Optics Express. 22 (4), 3968-3982 (2014).

5. Martínez-Fuentes, J. L., Moreno, I. Random technique to encode complex valued holograms with on axis reconstruction onto phase-only displays. Optics Express. 26 (5), 5875-5893 (2018).

6. Clark, T. W., Offer, R. F., Franke-Arnold, S., Arnold, A. S., Radwell, N. Comparison of beam generation techniques using a phase only spatial light modulator. Optics Express. 24 (6), 6249-6264 (2016).

7. Mendoza-Yero, O., Mínguez-Vega, G., Lancis, J. Encoding complex fields by using a phase-only optical element. Optics Letters. 39 (7), 1740-1743 (2014).

8. Yamaguchi, I., Zhang, T. Phase-shifting digital holography. Optics Letters. 22 (16), 1268-1270 (1997).

9. Shao, Y. et al. Addressable multiregional and multifocal multiphoton microscopy based on a spatial light modulator. Journal of Biomedical Optics. 17(3), 030505 (2012).

10. Mendoza-Yero, O., Carbonell-Leal, M., Mínguez-Vega, G., Lancis, J. Generation of multifocal irradiance patterns by using complex Fresnel holograms. Optics Letters. 43 (5), 1167-1170 (2018).

11. Kuang, Z. et al. Diffractive Multi-beam Ultra-fast Laser Micro-processing Using a Spatial Light Modulator (Invited Paper). Chinese Journal of Lasers. 36(12), 3093-3115 (2009).

12. Kuang, Z. et al. High throughput diffractive multi-beam femtosecond laser processing using a spatial light modulator. Applied Surface Science. 255, 2284-2289 (2008). 


\title{
Generation of multifocal irradiance patterns by using complex Fresnel holograms
}

\author{
Omel Mendoza-Yero, ${ }^{*}$ Miguel Carbonell-Leal, Gladys Mínguez-Vega, and Jesús Lancis \\ GROC UJI, Institute of New Imaging Technologies, Universitat Jaume I, 12071-Castelló, Spain \\ *Corresponding author: omendoza@fca.uji.es
}

Received 20 September 2017; revised 24 November 2017; accepted 6 February 2018; posted 7 February 2018 (Doc. ID 307632 );

published 28 February 2018

We experimentally demonstrate Fresnel holograms able to produce multifocal irradiance patterns with micrometric spatial resolution. These holograms are assessed from the coherent sum of multiple Fresnel lenses. The utilized encoded technique guarantees full control over the reconstructed irradiance patterns due to an optimal codification of the amplitude and phase information of the resulting complex field. From a practical point of view, a phase-only spatial light modulator is used in a couple of experiments addressed to obtain two- and three-dimensional distributions of focal points to excite both linear and non-linear optical phenomena. () 2018 Optical Society of America

OCIS codes: (050.1970) Diffractive optics; (120.5060) Phase modulation; (180.4315) Nonlinear microscopy; (230.6120) Spatial light modulators.

https://doi.org/10.1364/OL.43.001167

Diffractive phase optical elements (DPOEs) have been demonstrated as useful tools to get partial and sometimes full control over the behavior of laser beams in a wide variety of applications. For instance, the design of several types of phase-only filters [1-6] has found usefulness for optical encryption [4], correlation discrimination [5], or pulse shaping [6]. Other types of DPOEs have been proposed as a practical alternative to bulk optics for applications in which the generation of an array of focal spots is mandatory, such as parallel material processing [7], digital lensless holographic microscopy [8], second-harmonic generation [9], or multifilamentation [10]. Furthermore, the use of iterative Fourier transform algorithms [11-16] allows us to design DPOEs that can be utilized to create arbitrary holographic atom traps [15], or make spatial and temporal beam shaping $[10,16]$.

It is well known that DPOEs can be fabricated by means of the photolithographic technique or implemented onto spatial light modulators (SLMs) to work under either static or dynamic conditions. In particular, phase-only SLMs have demonstrated good performance in applications that demand high diffraction efficiency DPOEs, which also can be temporally modulated at refresh rates on the order of a few Hz. Hence, the development of efficient methods to encode the complex field of laser beams into a single-phase element is a key point for present and future applications.

In this context, a simple focusing diffractive element (FDE), i.e., a kinoform Fresnel lens, can be regarded as a simple Fresnel hologram which, after its reconstruction at the focal plane, generates a given irradiance point. It is apparent that a single FDE cannot be used to encode complex irradiance distributions, but combinations of them have been employed, i.e., in holographic femtosecond laser processing [17] or for the generation of Bessel beam arrays [18]. To this end, a set of FDEs has been encoded onto a Fresnel hologram to generate two- and threedimensional arrays of focal spots with high efficiency and numerical aperture. However, some characterization parameters of these irradiance points, i.e., energy, position or width, depend on, among other factors, laser beam characteristics, main features of the SLM, and particularly on the encoding method employed.

Regarding this last aspect, there are a wide number of reported encoding methods. For instance, in Ref. [18], the corresponding complex light fields are superposed, and the final Fresnel hologram is obtained after extracting the phase. In another approach, the hologram is determined after imposing certain conditions over the amplitude of the FDEs, compensating later unwanted interference effects by means of an optimization algorithm [17]. Additionally, an encoding method aimed to produce three-dimensional arrays of focused spots of light based on Damman gratings has also been demonstrated [19]. However, to the best of our knowledge, there is no report on methods to encode, by means of the double-phase hologram representation [20], the complex field obtained from the coherent sum of phase lenses. Note that, in comparison with other reported techniques, one able to reconstruct the complex field with accuracy on the order of the pixel size should improve the precision and quality of the focused spots.

In this Letter, we show a method to generate two- and threedimensional multifocal irradiance patterns of foci with precise positions at micrometric scale, and arbitrary spatial distributions. In particular, we experimentally demonstrate full control over positions, both in the transversal and axial coordinates of multiple irradiance points with a spatial resolution on the order of the pixel size of the SLM. Here, it should be mentioned that, in comparison with other well-known methods to generate 
multifocal points based only on the phase information of lenses, i.e., arrays or multiplexed lenses, the method proposed in this Letter fully encodes the amplitude and phase of the complex field obtained from the coherent sum of Fresnel lens functions. Then, from the reconstructed complex field, we are able to produce as many foci as their widths and the available energy allow. In addition, we can equalize the intensity of different foci by proper weighting of individual lens functions. To test its usefulness for applications in non-linear microscopy, we include an experiment for simultaneous multifocal excitation of a dendrite-shape distribution of 30 foci, with an average diameter of $1.8 \mu \mathrm{m}$ (measured following the $1 / e^{2}$ criterion for beam width determination), and located within a square spatial window of only $55 \times 55 \mu \mathrm{m}$ inside an uncoated Type 1 $\beta-\mathrm{BaB}_{2} \mathrm{O}_{4}(\mathrm{BBO})$ crystal.

In order to get further insight on the encoding method [21], the whole process is divided into three different steps. In the first step, the complex field $U(x, y)$ given by the coherent sum of $N$ weighted lens functions $K_{n}(x, y) e^{i \gamma_{n}(x, y)}$, with $n=1 \ldots N$, is determined. Here, the amplitude of each lens is denoted by $K_{n}(x, y)$, whereas their phases $\gamma_{n}(x, y)$ can be defined by the following expression:

$$
\gamma_{n}(x, y) \equiv \bmod \left\{\pi\left[\left(x-\xi_{n}\right)^{2}+\left(y-\eta_{n}\right)^{2}\right] /\left(\lambda f_{n}\right), 2 \pi\right\} .
$$

In Eq. (1), the terms $\xi_{n}$ and $\eta_{n}$ are the coordinates of the center of the lenses, $f_{n}$ represents their focal lengths, and the function $\bmod (p, q)$ gives the remainder of the division of $p$ by $q$. Please note that for $N>1$, both amplitude and phase of the complex field $U(x, y)$ depend also on the transversal coordinates $x, y$. Hence, to encode into a phase-only SLM not only the information of the phase corresponding to the complex field, but also that of its amplitude, we carry out the procedures described in the second and third steps.

In the second step, the complex field $U(x, y)$ is conveniently expressed as $U(x, y)=e^{i \theta_{1}(x, y)}+e^{i \theta_{2}(x, y)}$, where the phase elements $\theta_{1}(x, y)$ and $\theta_{2}(x, y)$ can be now calculated in terms of the amplitude and phase of the complex field $U(x, y)[20,21]$. In the last step, two checkerboard patterns $\mathrm{M}_{1}(x, y)$ and $\mathrm{M}_{2}(x, y)$, such as $\mathrm{M}_{1}(x, y)+\mathrm{M}_{2}(x, y)=1$, taken at the Nyquist limit, are used to sample the functions $e^{i \theta_{1}(x, y)}$ and $e^{i \theta_{2}(x, y)}$. As the checkerboard patterns do not overlap in the spatial domain, the following equality holds:

$$
\mathrm{M}_{1} e^{i \theta_{1}}+\mathrm{M}_{2} e^{i \theta_{2}}=e^{i\left(\mathrm{M}_{1} \theta_{1}+\mathrm{M}_{2} \theta_{2}\right)} .
$$

For clarity, in Eq. (2) the explicit dependence of all functions with $x$ and $y$ has been omitted. From Eq. (2), a single-phase element can be defined as $\alpha \equiv \mathrm{M}_{1} \theta_{1}+\mathrm{M}_{2} \theta_{2}$. Note that at this step of the process, the information from the uniform waves $e^{i \theta_{1}(x, y)}$ and $e^{i \theta_{2}(x, y)}$ that appears in $\alpha$ has not been added coherently, but it is spatially distributed with the checkerboard patterns $\mathrm{M}_{1}(x, y)$ and $\mathrm{M}_{2}(x, y)$. In order to do this sum, a $4 f$ imaging system, with a spatial filter, i.e., a hard iris, at the Fourier plane is employed. The complex Fresnel hologram is finally obtained at the output plane of the imaging system. Note that the spatial filtering process allows carrying out the coherent mix between both waves, as a common-path interferometer does. This represents a clear advantage over conventional two-arm interferometers in terms of alignment time as well as mechanical robustness. In addition, the Nyquist limit condition together with the small pixel width of the SLM $(8 \mu \mathrm{m})$ ensure that most sampled functions implemented in the SLM can be regarded, after simple interpolation, as an accurate representation of the original ones. Hence, the output irradiance patterns reconstructed from our complex Fresnel holograms can approach the positions of the corresponding focal points with a spatial resolution on the order of pixel width of the SLM. Furthermore, the peak intensity of focal points can be independently modified by changing the amplitude terms $K_{n}(x, y)$ associated to each lens function.

To experimentally test our proposal, we design a particular phase element $\alpha$ able to generate a three-dimensional distribution of irradiance points that resemble the form of the word UJI. Each character of the word was designed to be reconstructed at a different axial plane, so during the encoding process, three different focal lengths were selected $(400 \mathrm{~mm}$, $600 \mathrm{~mm}$, and $800 \mathrm{~mm}$ ). The design of the phase element $\alpha$ was carried out following the above-described encoding process, and consequently, the desired irradiance distribution of focal points was experimentally generated at the output plane of certain $4 f$ imaging system. Details of the whole optical setup are shown in Fig. 1 (upper part).

The laser beam emitted by a commercial femtosecond amplifier is sent to the SLM by using several silver mirrors. Before that, the laser beam passes through a set of neutral filters that allow us to control its energy. In addition, to get a spectrum centered at $800 \mathrm{~nm}$ having a bandwidth of about $10 \mathrm{~nm}$, the laser beam is filtered in the spectral domain with a bandpass filter (FB800-10 with full width at half-maximum $10 \pm 2 \mathrm{~nm}$ ). In this manner, we avoid unwanted chromatic effects due to the propagation and diffraction of the light at the different wavelengths. The beam is reflected from the SLM forming a small angle (less than $4 \mathrm{deg}$ ) with respect to the normal axis to the liquid crystal surface. After that, an imaging system composed of lenses $\mathrm{L}_{1}$ and $\mathrm{L}_{2}$ (with focal lengths $f_{1}=1 \mathrm{~m}$ and $f_{2}=0.5 \mathrm{~m}$, respectively) is employed to get the final complex Fresnel hologram at the output plane. To accurately locate the three different focal planes, the CCD camera sensor is mounted over a motorized stage. Owing to the axial demagnification $(1 / 4)$ of the imaging system, the locations of these planes are found at $100 \mathrm{~mm}, 150 \mathrm{~mm}$, and $200 \mathrm{~mm}$ away from the complex Fresnel hologram.

In Fig. 2, a three-dimensional view of the experimental irradiance distributions generated at the above-mentioned focal planes is shown. In addition, the central part of the designed phase element $\alpha$ is given in Fig. 2(a). However, for simplicity neither the $4 f$ optical system nor the complex Fresnel

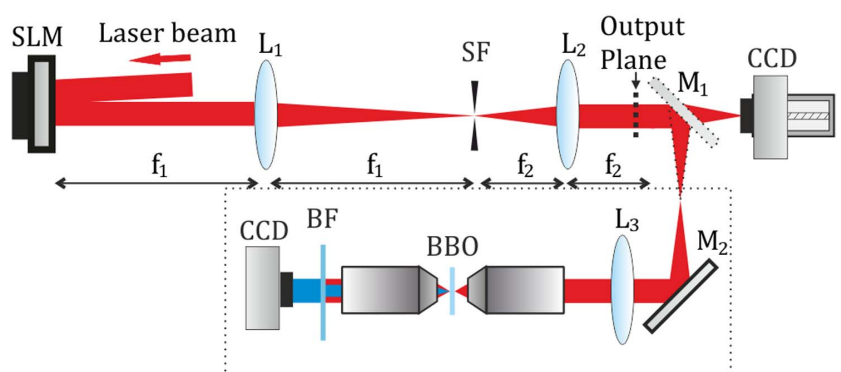

Fig. 1. Optical setup used to generate two- and three-dimensional distributions of focal points from a single-phase element at wavelengths centered at $800 \mathrm{~nm}$ (top part) and $400 \mathrm{~nm}$ (bottom part). In the figure, $L_{1}-L_{3}$, lenses; $M_{1}$ and $M_{2}$, silver mirrors; $B F$, blue filter; SF, spatial filter; $\mathrm{BBO}$, nonlinear crystal. 


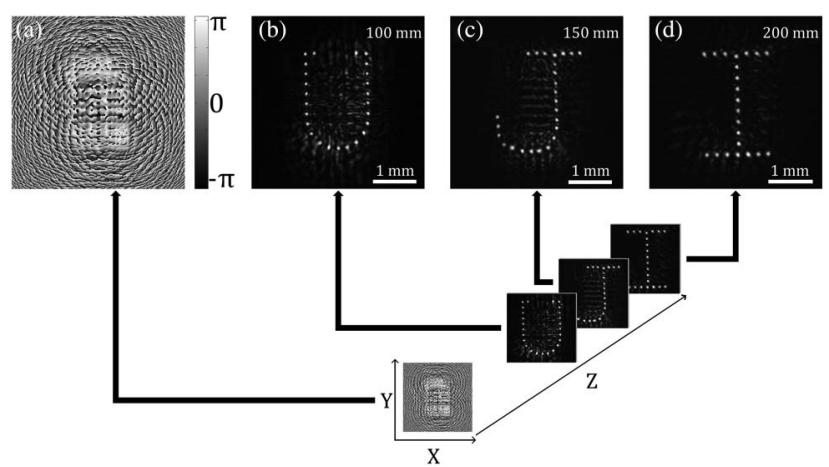

Fig. 2. User-defined distribution of focal points obtained at different axial planes (b)-(d) due to the diffraction of light from a singlephase element (a) encoded into the SLM. Each multifocal irradiance pattern was recorded within a spatial window of $4.4 \times 3.6 \mathrm{~mm}$.

hologram is represented. The first distribution of irradiance points with a U-shape is shown in Fig. 2(b), whereas the other two corresponding to the $J$ - and $I$-shapes are given in Figs. 2(c) and 2(d), respectively.

The whole pattern UJI is composed of 70 points, but owing to the different reconstruction planes of each character, the radii of the generated foci from the plane $z=100 \mathrm{~mm}$ to the plane $z=200 \mathrm{~mm}$ are slightly increased. At the first plane, shown in Fig. 2(b), these foci have an average measured radius of approximately $35 \mu \mathrm{m}$ (measured following the $1 / e^{2}$ criterion for beam width determination). For a Gaussian beam, the above increment $\Delta w$ of the radii from one plane to the next can be estimated using the following expression: $\Delta w=\lambda \mathrm{M}^{2} \Delta f /\left(\pi w_{0}\right) \cong$ $12 \mu \mathrm{m}$, where $\lambda=800 \mathrm{~nm}$ holds for the central wavelength of light, $\mathrm{M}^{2} \cong 2$ is the quality factor of our beam, $w_{0}=$ $2.16 \mathrm{~mm}$ is the radius of the beam at the complex Fresnel hologram, and $\Delta f=50 \mathrm{~mm}$ is the focal length difference between two consecutive planes. We also checked that the average measured radii $(48 \mu \mathrm{m}$ and $57 \mu \mathrm{m})$ at planes $z=150 \mathrm{~mm}$ and $z=200 \mathrm{~mm}$ agree well with the ones calculated by the above expression $(47 \mu \mathrm{m}$ and $59 \mu \mathrm{m})$.

The results shown in Fig. 2 reproduce well the expected distribution of foci at each plane. That is, after taking into account the demagnification of the $4 f$ optical system, the central position of each focus can be estimated by its corresponding pixel location within the SLM. In accordance with our model, the transversal coordinates of these pixels are denoted by $\left(\xi_{n}, \eta_{n}\right)$, with $n=1 \ldots N$. From the recorded images shown in Fig. 2, we found that actual locations of the central positions of foci differ, in all cases, less than 3 pixels from their expected positions. In addition, the relative peak intensities of the foci differs by as much as $20 \%$. We believe that small variations in the peak intensity and central positions of experimental foci can be originated, among other factors, by possible laser beam aberrations, or unwanted changes in the phase behavior of the SLM at different zones of its display. In addition, the calculus of the central positions of foci through the first-order moment of the corresponding irradiance distributions is also affected by the spatial resolution of the recording device.

On the other hand, as in principle our method can encode the complex field $U(x, y)$ obtained from the sum of a large number of lens functions, the number of foci that our method can generate is mainly determined by the minimum distance among them to avoid their overlapping and the available laser beam energy. From a practical point of view, possible limitations due to the above parameters are usually less restrictive than those found in conventional arrays of lenses in which both the amount of lenses and their diameters are linked parameters (the larger the number of encoded lenses, the lower their diameters).

The previous experiment showed some of the main characteristics of the proposed method. However, we believe that this method also can be useful for other practical applications, including laser material processing or biomedical optics. In this direction, we carry out a proof-of-concept experiment in the field of non-linear optical microscopy. Its optical setup is mostly represented in the bottom part of Fig. 1, within an area delimited by a dotted rectangle. The idea of the experiment is to demonstrate controlled multifocal second-harmonic patterns at micrometric scale with an arbitrary distribution of the focal spots. These kinds of illumination patterns could be used, e.g., to excite micrometric sections of a biological sample. Here, following the same encoding procedure described before, we generate a distribution of 30 foci that resemble the structure of certain terminal axons of a common neuron. In this case, the experimental results corresponding to both fundamental (light with central wavelength at $800 \mathrm{~nm}$ ) and second-harmonic multifocal irradiance patterns are shown in Figs. 3(a) and 3(b), respectively.

In particular, the relative peak intensity differences of foci generated from the fundamental beam, shown in Fig. 3(a), are less than $20 \%$, and their corresponding spatial locations have discrepancies with respect to the expected ones all below 3 pixels. This distribution of foci is imaged into an uncoated Type $1 \mathrm{BBO}$ crystal with dimensions $10 \mathrm{~mm} \times 10 \mathrm{~mm} \times$ $0.01 \mathrm{~mm}$ with the help of the lens $\mathrm{L}_{3}$ of focal length $f_{3}=$ $400 \mathrm{~mm}$ and a $20 \times$ microscopy objective (see bottom part of Fig. 1). Then, the second-harmonic signal emitted by the excited medium is recorded with the same CCD camera used before, but now located at the output image plane of a second $50 \times$ microscopy objective.

For this experiment, we remove the bandpass filter from the optical setup. In addition, the material dispersion introduced by some of the components of the optical system during the propagation of the ultrashort pulse until the $\mathrm{BBO}$ crystal was pre-compensated with the help of a commercially available dispersion compensation module (FASTLITE-Dazzler). Finally, the non-converted fundamental light after the BBO

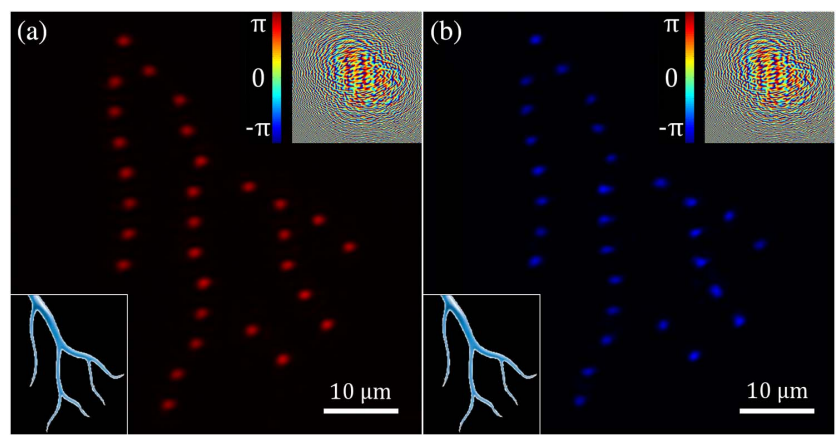

Fig. 3. Recorded images of the user-defined fundamental (a) and second-harmonic (b) spatial distribution of 30 focal points. Each image was recorded within a square spatial window of $55 \times 55 \mu \mathrm{m}$. 
crystal is suppressed with a bandpass filter (BG-39). The resulting second-harmonic irradiance pattern is now shown in Fig. 3(b). Furthermore, a section of the phase element $\alpha$ used in this experiment is given as an inset in the top-right part of Fig. 3 .

Again, the experimental results shown in Fig. 3(b) are in agreement with the expected distribution of foci. The positions of the second-harmonic foci coincide with the expected ones with an error on the order of the pixel size of the SLM, and their relative peak intensities differs less than $30 \%$. In this case, peak intensity equalization is more critical to achieve, among other reasons, due to the different phase matching conditions associated with the generation of each focus, as well as the different phase response of the SLM to the wide frequency content of the ultrashort pulse. Here, it also should be noted that the second-harmonic signal depends on the square of the incident irradiance, so small variations in the intensity of excitation spots can significantly change the peak intensity distribution of second-harmonic foci.

From Fig. 3, one can also realize that all foci are located within a spatial region of only $55 \times 55 \mu \mathrm{m}$. In addition, their measured diameters are less than $2 \mu \mathrm{m}$. Note that the abovementioned sizes are on the order of most cell sizes, which typically range from $10 \mu \mathrm{m}$ to $50 \mu \mathrm{m}$, e.g., the soma of a neuron. Irradiance patterns like those shown in Fig. 3 have been proved to be crucial for carrying out simultaneous and selective illumination of biological samples, which is the key point to reduce the excitation time in wide-field microscopy.

In this Letter, we have experimentally shown the potential of our encoding method to generate user-defined irradiance patterns composed of multiple focal spots. From our point of view, the main limitation of the method lies in the impossibility to work at frequency ranges greater than the refresh time of the phase-only SLMs, which is typically on the order of a few tens of hertz. In addition, the complex fields are encoded by using two phase functions sampled with half of their original values. Hence, the efficiency of the reconstructed holograms is theoretically reduced by a factor of 4 , with respect to the ideal case (i.e., the reconstruction of the hologram by interference between two uniform waves).

In contrast, in comparison with other well-known methods, e.g., those based on two-dimensional arrays of lenses or spatial multiplexing of lenses, with our proposal, the number of achievable foci is mainly determined by the available laser energy and the specified distance among them. Furthermore, the accurate reproduction of the complex field obtained from the coherent sum of complex lens functions ensures not only micrometric precision localization of foci's centroids, but also a fine equalization of their peak intensity. We believe that the above-introduced method might be useful in applications such as multi-photon microscopy or laser micro-processing.

Funding. Generalitat Valenciana (AICO/2016/036, PROMETEO 2016-079); Universitat Jaume I (UJI) (UJIB2016-19); Ministerio de Economía y Competitividad (MINECO) (FIS2016-75618-R).

Acknowledgment. The authors are very grateful to the SCIC of the Universitat Jaume I for the use of the femtosecond laser.

\section{REFERENCES}

1. J. A. Davis, D. M. Cottrell, J. Campos, M. J. Yzuel, and I. Moreno, Appl. Opt. 38, 5004 (1999).

2. V. Arrizón, Opt. Lett. 28, 2521 (2003).

3. R. W. Cohn and M. Liang, Appl. Opt. 33, 4406 (1994).

4. P. C. Mogensen and J. Glückstad, Opt. Lett. 25, 566 (2000).

5. A. A. S. Awwal, M. A. Karim, and S. R. Jahan, Appl. Opt. 29, 233 (1990).

6. A. M. Weiner, S. Oudin, D. E. Leaird, and D. H. Reitze, J. Opt. Soc Am. A 10, 1112 (1993).

7. S. Torres-Peiró, J. González-Ausejo, O. Mendoza-Yero, G. MínguezVega, P. Andrés, and J. Lancis, Opt. Express 21, 31830 (2013).

8. O. Mendoza-Yero, E. Tajahuerce, J. Lancis, and J. Garcia-Sucerquia, Opt. Lett. 38, 2107 (2013).

9. G. Mínguez-Vega, C. Romero, O. Mendoza-Yero, J. R. Vázquez de Aldana, R. Borrego-Varillas, C. Méndez, P. Andrés, J. Lancis, V. Climent, and L. Roso, Opt. Lett. 35, 3694 (2010).

10. O. Mendoza-Yero, M. Carbonell-Leal, C. Doñate-Buendía, G. Mínguez-Vega, and J. Lancis, Opt. Express 24, 15307 (2016).

11. R. W. Gerchberg and W. O. Saxton, Optik 35, 237 (1972).

12. G. Z. Yang, B. Z. Dong, B. Y. Gu, J. Y. Zhuang, and O. K. Ersoy, Appl. Opt. 33, 209 (1994).

13. J. R. Fienup, Appl. Opt. 21, 2758 (1982).

14. F. Wyrowski and O. Bryngdahl, J. Opt. Soc. Am. A 5, 1058 (1988).

15. M. Pasienski and B. DeMarco, Opt. Express 16, 2176 (2008).

16. O. Ripoll, V. Kettunen, and H. P. Herzig, Opt. Eng. 43, 2549 (2004).

17. S. Hasegawa, Y. Hayasaki, and N. Nishida, Opt. Lett. 31, 1705 (2006).

18. R. Bowman, N. Muller, X. Zambrana-Puyalto, O. Jedrkiewicz, P. Di-Trapani, and M. J. Padgett, Eur. Phys. J. Spec. Top. 199, 159 (2011).

19. J. A. Davis, I. Moreno, J. L. Martínez, T. J. Hernandez, and D. M. Cottrell, Appl. Opt. 50, 3653 (2011).

20. C. K. Hsueh and A. A. Sawchuk, Appl. Opt. 17, 3874 (1978).

21. O. Mendoza-Yero, G. Mínguez-Vega, and J. Lancis, Opt. Lett. 39, 1740 (2014). 


\title{
Encoding of arbitrary micrometric complex illumination patterns with reduced speckle
}

\author{
Miguel Carbonell-Leal, Gladys Mínguez-Vega, Jesús Lancis, And \\ OMEL MENDOZA-YeRO* \\ Institut de Noves Tecnologies de la Imatge (INIT), Universitat Jaume I, 12080 Castelló, Spain \\ *omendoza@fca.uji.es
}

\begin{abstract}
In nonlinear microscopy, phase-only spatial light modulators (SLMs) allow achieving simultaneous two-photon excitation and fluorescence emission from specific regionof-interests (ROIs). However, as iterative Fourier transform algorithms (IFTAs) can only approximate the illumination of selected ROIs, both image formation and/or signal acquisition can be largely affected by the spatial irregularities of the illumination patterns and the speckle noise. To overcome these limitations, we propose an alternative complex illumination method (CIM) able to generate simultaneous excitation of large-area ROIs with full control over the amplitude and phase of light and reduced speckle. As a proof-of-concept we experimentally demonstrate single-photon and second harmonic generation (SHG) with structured illumination over large-area ROIs.
\end{abstract}

(C) 2019 Optical Society of America under the terms of the OSA Open Access Publishing Agreement

\section{Introduction}

In some recent introduced multiphoton microscopy techniques, simultaneous excitation and signal collection from multiple specific cell populations have become into key tools for monitoring the cellular activity [1-3]. This basically happens because multiple millisecond time response signals e.g., fluorescence-lifetime signals, produced by many cellular ensembles cannot be acquired by means of conventional scanning methods. For instance, in point by point scanning methods the temporal resolution is limited by the signal-to noise ratio, which is closerelated to the pixel dwell time. That is, the longer the pixel dwell time, the higher the signalto-noise ratio but consequently, temporal resolution gets worse. An alternative illumination technique employed to reduce the acquisition time relies on the parallel excitation of the cellular targets by using multifocal irradiance patterns. On this topic, multifocal multiphoton microscopy can be carried out by several methods including, but not limited to, the use of microlens arrays [4,5], beam splitting [6], or Fresnel holograms [7]. However, it is apparent that multifocal illumination allows us to excite only focal-size sites in the cells, which certainly limits the acquired information e.g., voltage signals, to those focal regions determined by the spatial features of the excitation foci.

On the other hand, the combination of wide-field illumination techniques with highsensitivity cameras (e.g., TE-cooled, ultra-sensitive photon detecting, electron multiplying charge-coupled camera) can be regarded as a prominent solution to simultaneously excite and collect not just from focal-size sites within the cells, but also from large-area ROIs of them. In this case all ROIs of the biological cells delimited by the numerical aperture of the objective could be turned into single-photon fluorescence signals, although it is less likely to induce multiphoton absorption and fluorescence emission due to both the exponential intensity requirements of nonlinear processes and the spreading of the laser power over the extended regions. Hence, instead of conventional mode locked Ti: sapphire laser oscillators, additional chirped pulse amplification stages with average power capabilities up to several watts and repetition rates of tens of $\mathrm{kHz}$ become better candidates of choice for performing wide-field multiphoton microscopy $[8,9]$. 
In this context, commercially available SLMs have allowed for simultaneous excitation of several ROIs within a cellular ensemble thanks to a proper optical manipulation of ultrashort laser pulses [2,10-20]. Here, if one takes a look at the wide variety of reported illumination methods, those ones based on the use of phase-only SLMs seems to stand out because of their potential to manage light with relatively high throughputs. In particular, wide-field nonlinear excitation has been experimentally demonstrated by the coherent reconstruction of computergenerated holograms (CGHs) at the sample plane. In this case, although long part of the available energy can be transferred to specific ROIs within the cellular ensemble, there still exist several problems to face out. For instance, unwanted effects like angular dispersion or chromatic aberrations may cause spatiotemporal distortions of the reconstructed hologram, which clearly decrease the signal-to-noise ratio and harm the quality of the obtained cell image. In addition, the coherent noise (speckle) associated with the superposition of coherent light with different path lengths prevents wide-field excitation based on CGHs to be wellaccomplished over other ROIs different than the line-shaped illumination regions typically reported in the literature $[7,10]$. Therefore, under coherent illumination, the reconstruction of CGHs is currently unable to generate spatially homogeneous irradiance patterns over ROIs with dimensions on the order of the typical cellular-sizes, not to mention its inefficiency to precisely manipulate the amount of energy put into them e.g., to generate multiple-intensity level illumination patterns. At this point, improved versions of the well-known GerchbergSaxton algorithm [21] have allowed both speckle reduction and phase control of 1-D illumination patterns $[11,22]$. In addition, some recently reported CGH-based methods can generate controllable illumination patterns with multiple energy levels, but without phase control [23]. So, as far as we known, there is no report on the utilization of proper 2-D illumination methods that give us high accuracy and complete control over the amplitude and phase of the light without speckle noise.

In this manuscript we propose an interferometric CIM able to induce linear and nonlinear excitation of multiple cellular-size ROIs with full and independent control over the amplitude and phase of the light at the sample plane. Its ability to precisely modify the complex field of the spatially coherent light emitted by a femtosecond laser is experimentally shown to be crucial to obtain not only homogenous irradiance patterns (without speckle) over different ROIs, but also multiple-intensity levels with arbitrary phase content in all cases. To show the usefulness of our method to generate controllable light patterns, we experimentally demonstrate single photon illumination/excitation of a certain micrometric ROI by using different predefined complex field patterns. In addition, another proof-of-concept experiment, this time addressed to induce SHG of a specific ROI within a Type $1 \beta-\mathrm{BaB}_{2} \mathrm{O}_{4}$ (BBO) crystal is also shown. All optical control of the illumination patterns is carried out with a single phase element implemented into a phase-only SLM. The phase element is computer generated by using a complex field encoding method based on double-phase hologram theory that was previously reported [12]. Some results achieved with our CIM were compared with similar ones obtained by the reconstruction of CGHs.

\section{Basics of the complex illumination method}

In this section we introduce the theory underlying the CIM. The corresponding setup is basically composed of two consecutive optical modules that we refer throughout the whole manuscript as complex field encoding module (CFEM) and optical demagnifying module (ODM), respectively, see Fig. 1.

When the light passes through the CFEM, its optical wavefront is properly phase modulated to get user-defined complex fields at the output plane. In accordance with the theory [12], any two-dimensional complex field expressed in the form $U(x, y)=A(x, y) e^{i \varphi(x, y)}$, where $A(x, y)$ and $\varphi(x, y)$ hold for the amplitude and phase functions, respectively, can be rewritten as: 


$$
U(x, y)=B e^{i \theta(x, y)}+B e^{i \vartheta(x, y)} .
$$

In Eq. (1), $B=A_{\max } / 2$ is an amplitude constant term (it does not depends on the transversal coordinates $x, y), A_{\max }$ is the maximum value of $A(x, y)$, and the new phase functions $\theta(x, y)$ and $\vartheta(x, y)$ can be calculated from $A(x, y)$ and $\varphi(x, y)$ by using the expressions:

$$
\begin{aligned}
& \theta(x, y)=\varphi(x, y)+\cos ^{-1}\left[\mathrm{~A}(\mathrm{x}, \mathrm{y}) / \mathrm{A}_{\max }\right] . \\
& \vartheta(x, y)=\varphi(x, y)-\cos ^{-1}\left[A(x, y) / A_{\max }\right] .
\end{aligned}
$$

From a physical point of view (for $A_{\max }=2$ ), Eqs. (1-3) mean that the complex field $U(x, y)$ can be retrieved from the coherent interference of the two uniform waves $e^{i \theta(x, y)}$ and $e^{i,(x, y)}$. Such interference can be carried out by means of a common-path interferometer made up of a spatially filtered $4 \mathrm{f}$ optical system. The screen of a phase-only SLM is placed at the input plane of the CFEM, and the following phase element $\alpha(x, y)$ is implemented on the SLM

$$
\alpha(x, y)=M_{1}(x, y) \theta(x, y)+M_{2}(x, y) \vartheta(x, y) .
$$

The phase element $\alpha(x, y)$ is derived from the transversal spatial mapping of $e^{i \theta(x, y)}$ and $e^{i,(x, y)}$ with two-dimensional binary gratings $M_{1}(x, y)$ and $M_{2}(x, y)$ (checkerboard patterns) taken at the Nyquist limit, that is

$$
M_{1}(x, y) e^{i \theta(x, y)}+M_{2}(x, y) e^{i \vartheta(x, y)}=e^{i \alpha(x, y)} .
$$

The checkerboard patterns fulfill the complementary condition $M_{1}(x, y)+M_{2}(x, y)=1$. Here, it should be noted that, by itself, send the phase element $\alpha(x, y)$ to the SLM does not guaranties the interference of both uniform waves. This is carried out by means of a suited spatial filter placed at the Fourier plane of the imaging system. It can be shown that if we use a filter to block all diffraction orders but the zero one, we are able to exactly retrieve the full spectrum of the original complex field at the Fourier plane [12]. Consequently, at the output plane of the CFEM we find the convolution of the magnified spatially reversed complex field with the Fourier transform of the filter. It means that full amplitude and phase information of the complex field can be retrieved at the output plane, except for a certain loss of spatial resolution due to the convolution operation.

On the other hand, the ODM allows us to proper rescale, onto the sample plane, the complex field generated at the output plane of the CFEM. The ODM is composed of a refractive achromatic lens with long-distance focal length and an infinity corrected microscope objective, which generate a demagnified image of the reconstructed complex field onto the sample plane. In Fig. 1, the whole optical setup corresponding to the CIM is shown.

\section{Single photon experiments}

To demonstrate, as a proof-of-concept, the ability of the method to generate arbitrary complex illumination patterns at micrometric scale, we carried out several single photon experiments. The employed optical setup is shown in Fig. 1. Ultrashort pulses emitted by a Ti: Sapphire amplifier laser (30 fs amplitude full-width at half maximum (FWHM), central wavelength 800 $\mathrm{nm}$, bandwidth of $50 \mathrm{~nm}$ FWHM, maximum energy per pulse of $0.8 \mathrm{~mJ}$, and $1 \mathrm{kHz}$ repetition rate) are employed as a light source. The SLM (HOLOEYE PLUTO, resolution 1920x1080, and pixel pitch $8 \mu \mathrm{m}$ ) used in our experiments was previously calibrated [24] for a significant set of frequency components of the light pulse with the help of three bandpass filters of $10 \mathrm{~nm}$ FWHM. The similar phase response curves (calibration curves) obtained for the three different spectral lines allow performing wavefront modulation of ultrashort pulses with a single phase element $\alpha(x, y)$. In addition, to mitigate the effect of abrupt spatial variations in the phase 
modulation [25] (pixel crosstalk effects), the two-dimensional binary gratings $M_{1}(x, y)$ and $M_{2}(x, y)$ were constructed with $4 \times 4$ pixel-cells.

In the optical setup shown in Fig. 1, the light is sent to the SLM by using silver mirrors and reflected from them forming a small angle (about 3 degrees) with respect to the normal incidence. In these conditions, the desired complex field $U(x, y)$ is reconstructed at the output plane of the CFEM. The CFEM is made up of a couple refractive lenses $\left(\mathrm{L}_{1}\right.$ and $\left.\mathrm{L}_{2}\right)$ with focal lengths $\mathrm{L}_{1}=1000 \mathrm{~mm}$ and $\mathrm{L}_{2}=500 \mathrm{~mm}$, which result in a transversal magnification of $1 / 2$ at its output plane. In addition, a circular iris is placed at the Fourier plane to filter all diffraction orders but the zero one. Finally, the ODM allows the complex field illumination $U(x, y)$ to be imaged into the sample plane.

A rough coupling between the size of the illumination pattern and that of the sample is carried out with a proper selection of optical components of the ODM. In our experiment, it is made up of an achromatic lens $\left(\mathrm{L}_{3}\right)$ of focal length $400 \mathrm{~mm}$ and a 20x infinity-corrected microscope objective $\left(\mathrm{MO}_{1}\right)$, which led to a total demagnification of about 1/73 at the sample plane. The transmitted light is collected with the help of a 50x infinity-corrected microscope objective $\left(\mathrm{MO}_{2}\right)$ and sent to a CCD camera (Basler avA1600-50gm), where the image of the sample is recorded (see Fig. 1). To adjust the intensity of the light at the sample plane a set of broadband neutral filters was employed. A more accurate selection of the illumination zone over the sample is done later once the specific ROIs are defined. At this point it is possible to establish the main features of the illumination pattern $U(x, y)$. For instance, its amplitude function $A(x, y)$ can be set by means of a multilevel spatial energy distribution, or its phase function $\varphi(x, y)$ by using a proper phase distribution to compensate for specimen-induced or microscope objective spherical aberrations.

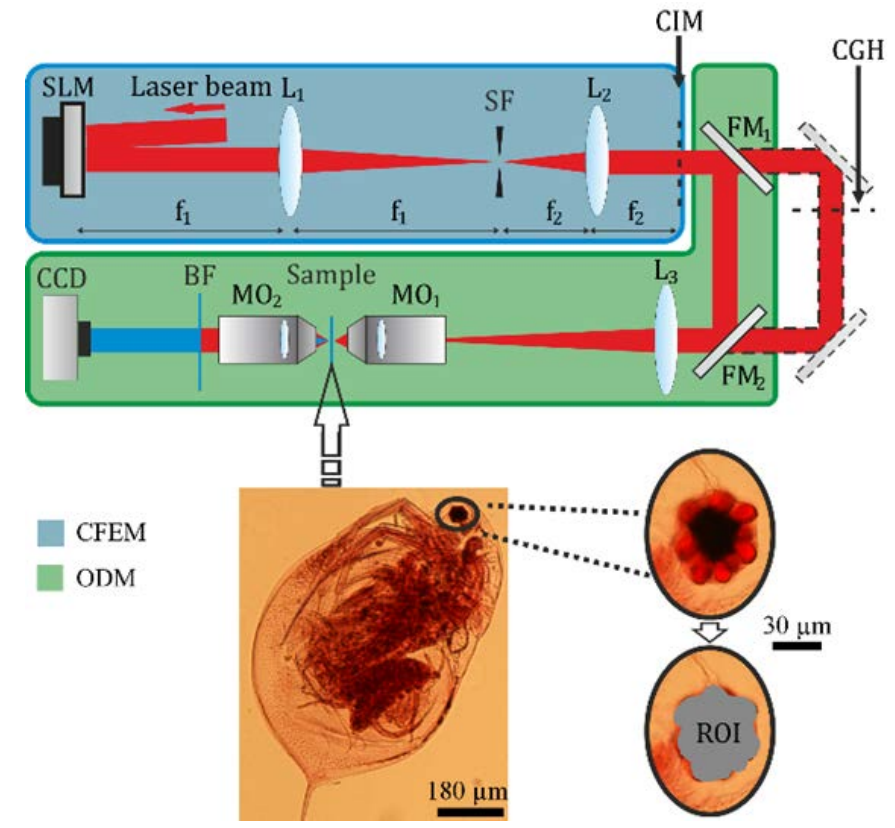

Fig. 1. Optical setup. Complex illumination method (CIM) implemented by means of two optical modules: complex field encoding module (CFEM, blue region) used to generate the arbitrary complex patterns, and optical demagnifying module (ODM, green region) used to adjust the size of the pattern at the sample plane. In order to properly set the position of the utilized CGHs, a delay line is added at the right part of the setup. Bottom part shows an image of the biological specimen (water flea) used to define a ROI. 
In Fig. 2, an example of a complex illumination pattern is shown. In this case, we simulate a ROI within a Daphnia specimen (water flea) that coincides with its eye. Note that, such ROI has approximate dimensions of $60 \times 60 \mu \mathrm{m}^{2}$. The complex field given in top part of Fig. 2 is characterized by an amplitude pattern having four different energy levels distributed among quadrants within the eye, whereas its spatial phase is given by the analytical function $\varphi(x, y)=\pi / 2 \sin [2 \pi x /(5 \lambda)] \cos [2 \pi y /(5 \lambda)]$. From the above-defined amplitude and phase parameters we can calculate the phase element $\alpha(x, y)$ used to reconstruct the expected complex field. To do that, we follow the procedure described in section 2, also represented schematically in central part of Fig. 2. Here, to clearly see the roll of sampling gratings $M_{1}(x, y)$ and $M_{2}(x, y)$ on this procedure, the phase element $\alpha(x, y)$ was represented with pixel-cells of 60x60. Polarization-based phase shifting technique [26] is applied to measure the amplitude and phase of the complex illumination pattern at the sample plane. This phase shifting technique can be accomplished by simple addition of a couple of polarizationdependent optical elements e.g., two polarizers. Specifically, a broadband halfwave plate (EKSMA OPTICS 460-4215) was placed before the SLM plane to rotate the original direction of polarization of all frequency components of the ultrashort pulse 45 degrees with respect to the SLM director orientation. This allows only part of the incident light to be phase modulated (object beam), while the remaining one (reference beam) is not diffracted. In order to generate four different phase shifting interferograms at the sample plane, uniform phases with steps of $\pi / 2$ radians were added to the phase element $\alpha(x, y)$. The interferograms are formed after recombining both reference and object beams with the help of a broadband linear polarizer (EKSMA OPTICS 420 0526M), this time located before the 20x microscope objective $\mathrm{MO}_{1}$. In practice, the position of the rotational angle with respect to SLM director orientation determines the amount of diffracted and non-diffracted light within the interferograms. In the bottom-central part of Fig. 2, the interferograms measured for the phase steps $0, \pi / 2, \pi$, and $3 \pi / 2$ are shown. These interferograms were recorded without moving the position of the camera in the optical setup. The experimental amplitude and phase patterns determined from the above phase shifting interferograms are given in the bottom part of Fig. 2. It is apparent that experimental results are in good agreement with the theory. However, as it might be expected due to the convolution operation (see section 2) and the used pixel-cell, the spatial resolution of recorded amplitude and phase patterns is a few pixels lower than corresponding theoretical ones. Even so, the root-mean-square error (RMSE) between theory and experiment yields discrepancies of $6.8 \%$ and $14 \%$ for amplitude and phase images, respectively.

Now, we test the usefulness of the CIM to synthetize amplitude-only irradiance patterns at micrometric scale. On one side, we are interested in the generation of spatially uniform irradiance patterns for simultaneous wide-field illumination. Note that the excitation of extended ROIs with non-uniform irradiance patterns may cause image blurring due to different signal responses of similar biological components. In wide-field two-photon microscopy this is crucial because the recorded signal depends on the square of the light intensity. In other cases, simultaneous excitation of extended ROIs (not by multifocal excitation) through beam shaping of coherent radiation cannot be accomplished without coherent noise (speckle). 


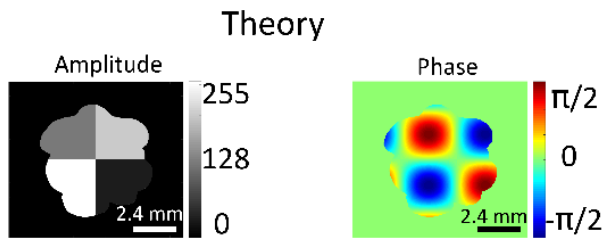

Encoding method

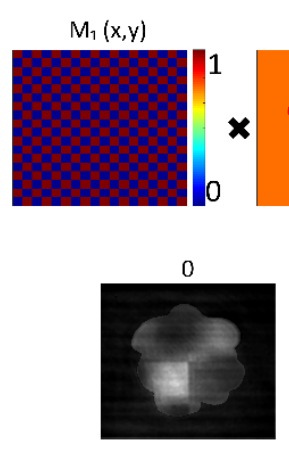

$\theta(x, y)$ $M_{2}(x, y)$

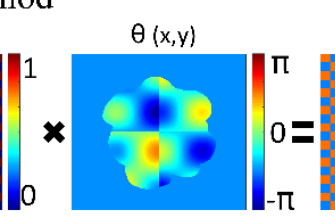
$a(x, y)$

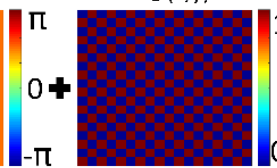

Phase-shifted interferograms
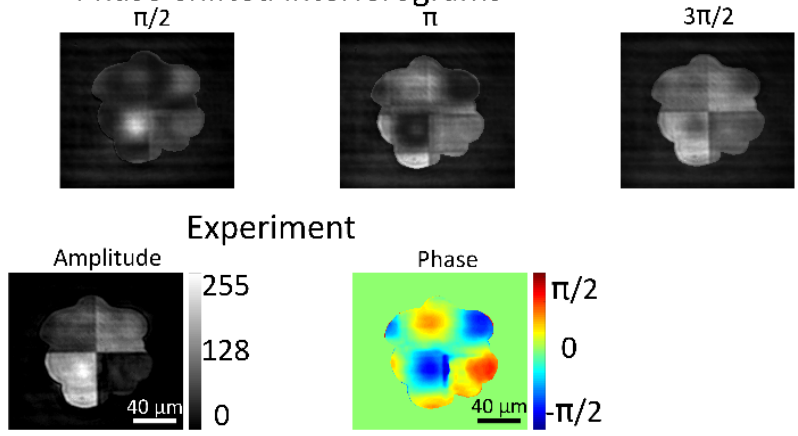

Fig. 2. Generic example: measured amplitude and phase of a given complex field at the SLM (top) and sample (bottom) planes. Related CIM images and measured interferograms are also shown (central part). Scales are included as insets at the right-bottom part of each irradiance pattern.

In contrast, by applying the proposed CIM we obtain spatial uniform illumination of extended ROIs without speckle (please, see top-right part of Fig. 3). In this experiment, as before, we select a ROI whose shape is determined by the borders of the Daphnia 'eye. The first column of Fig. 3 shows the phase elements $\alpha(x, y)$ represented with a pixel-cell of $6 \times 6$. Here, the RMSE between theory and experiment yields discrepancies of $7 \%$. The spatial uniformity of the recorded pattern mainly depends on the spatial profile of the laser beam at the SLM plane, and also to a lesser extent, of optical irregularities (scratches in mirrors or lenses, unwanted dust on the surface of optical components, etc.). Furthermore, as this irradiance pattern is basically constructed by conjugating the SLM plane with the sample plane, measured irradiance patterns are free from coherent noise.

On the other side, we push the CIM to the limit by implementing irradiance patterns with multiple energy levels and complex spatial structure. To this end, we reproduce inside of the ROI given by the Daphnia's eye (about $60 x 60 \mu^{2}$ only) a mushroom image. After a visual comparison of results shown in Fig. 3, one can realize that the recorded irradiance pattern reproduces very well the expected one. In fact, we are able to spatially resolve image details in the sample plane of the order of $5 \mu \mathrm{m}$ i.e., the white spots. Here, it should mentioned that the minimum feature size of our system is about $0.87 \mu \mathrm{m}$. In this case, the RMSE between theory and experiment yields discrepancies of $10 \%$. With this experiment, we want to show the potential of the CIM to manage different energy levels of excitation simultaneously. It is apparent that different constituents of cells and/or fluorophores might imply different excitation thresholds. So, in principle, one can think in setting energy-custom illumination patterns depending on the nature of the biological sample. Additionally, this fine control over 
the energy content of the illumination pattern can be also useful to adjust the energy levels over the whole sample without the necessity of using neutral filters.

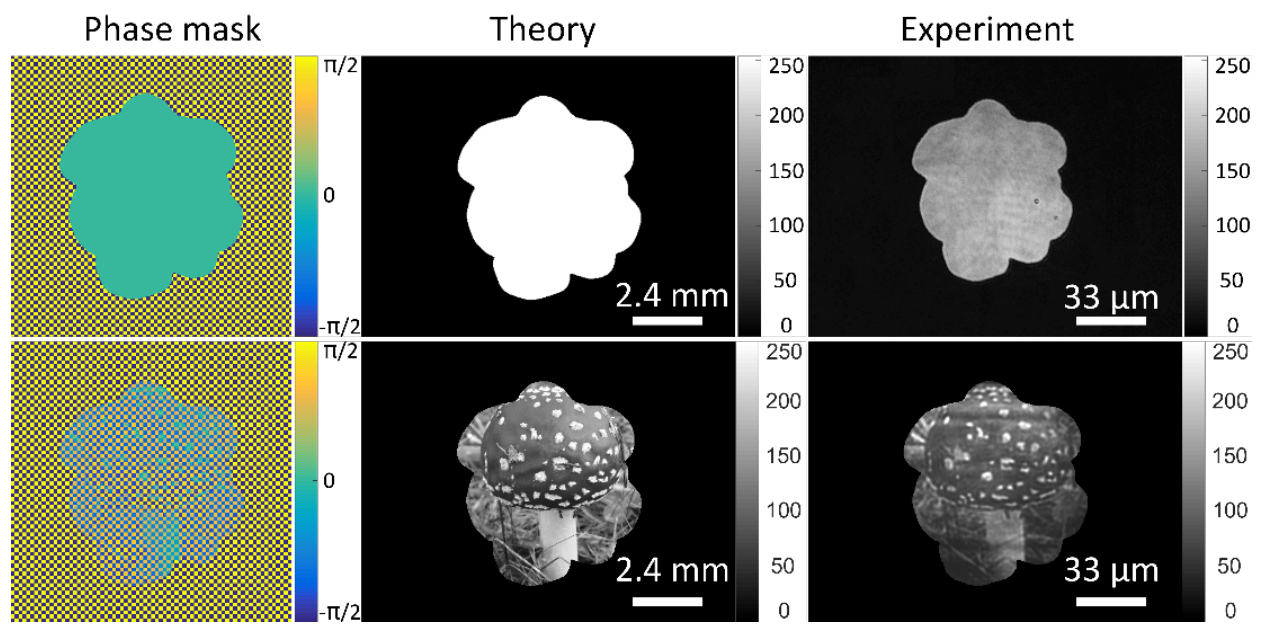

Fig. 3. Theoretical and experimental amplitude-only irradiance patterns at the SLM and sample planes, respectively. Scales are included as insets at the right-bottom part of each irradiance pattern. In the first column, corresponding phase elements, represented with a pixel cell of 6x6, are also shown.

In order to highlight the role of the phase in practical tasks we carried out real optical aberration corrections using the CIM. For this purpose, we constructed and introduced a Michelson interferometer within the CFEM to measure the phase. In this setup, the light impinges perpendicular to the liquid crystal display after being divided into two similar beamlets with a broadband beam splitter. In the first arm of the interferometer, the light (object beam) goes to the SLM and is reflected back to the CFEM via beam splitter, whereas in the second arm, the light (reference beam) follows a similar optical path, but is reflected by a silver mirror. This mirror can be slightly moved (delay line) to adjust the path length of both arms. The phase is measured at the output plane of the CFEM by applying the Polarization-based phase shifting technique [26] in a similar manner as described before. However, in contrast to previously common-path setup, this type of interferometer is able to determine the optical aberrations of the optical system. The conjugated measured phase is encoded with the CIM algorithm to get both significant correction for optical aberrations and synthesis of desired complex field, simultaneously.

In Fig. 4 some specific results are shown. In the simplest example, a complex field made up of a uniform amplitude and a flat phase is encoded into the SLM, see Fig. 4(a). The amplitude pattern, given by the borders of Daphnia's eye, and shown as an inset in the leftbottom part of each image, is the same in all cases. Although the encoded phase is flat, in practice the retrieved phase from the phase shifting interferograms shows clear optical aberrations, see Fig. 4(b). These aberrations can be largely compensated if we take them into account within the CIM algorithm, as shown in Fig. 4(c). To do that, it is sufficient to encode the conjugated of the measured optical aberrations together with the complex pattern. This aberration correction task is usually necessary to better approach the reconstructed complex field to the expected one. Last example shown in Fig. 4(d)-(f) illustrates this situation. In this case, the desired phase pattern is originally given in Fig. 4(d), whereas the real retrieved phase is distorted by optical aberrations, see Fig. 4(e). By using the CIM we can correct most optical aberrations, achieving the phase pattern given in Fig. 4(f), which is closer to the desired one. 

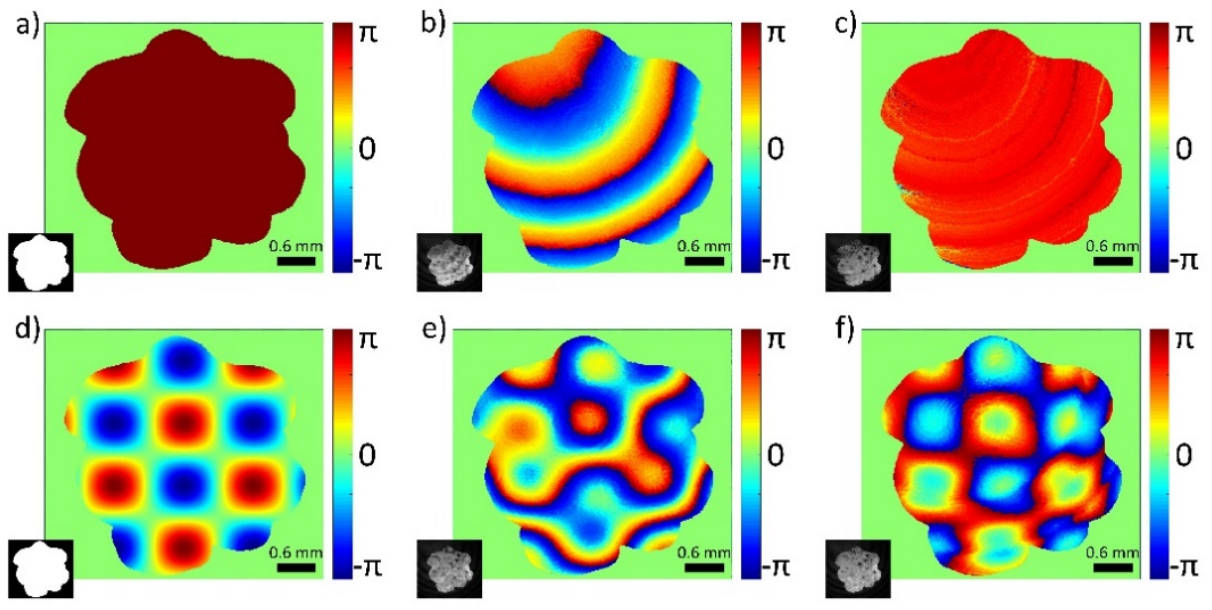

Fig. 4. Two examples of compensation for real optical aberrations with the CIM. The measured amplitude patterns are included as insets at the left-bottom parts of each image.

In most aberration compensation techniques based on a phase-only SLM, the liquid crystal display is used just to implement the necessary conjugated phase to compensate for previously measured optical aberrations. In contrast, the encoding algorithm associated with the CIM allows us to perform wavefront correction and manipulate the amplitude of the complex field, simultaneously.

\section{Non-linear excitation experiment}

In this section we experimentally demonstrate, also as a proof-of-concept, nonlinear excitation phenomena of ROIs at micrometric scale. In particular, we show that our CIM can generate simultaneous and controllable second harmonic signal within a selected ROI. The chosen complex illumination pattern is made up of a uniform amplitude and a proper phase able to compensate few optical aberrations found at the sample plane. This phase was determined as the conjugated of that one obtained with a Shack-Hartmann wavefront sensor after sending a matrix of zero radians to the SLM. The wavefront sensor is placed at the same plane of the camera with the help of an additional beam splitter (not shown in Fig. 1). The ROI resembles again the structure of the eye of a Daphnia (similar to that shown in top part of Fig. 3). In addition, to compare the proposed CIM with a well-established illumination method, we include in the optical setup a delay line for synthetizing similar amplitude illumination patterns by means of CGHs. For this purpose the optical setup is designed such that the reconstructed CGH appears at the same distance from the lens $\mathrm{L}_{3}$ as the complex field $U(x, y)$ does. The CGHs were designed following the Gerchberg-Saxton IFTA [21], developed in two different stages. In the first stage we perform iterations by applying only phase freedom, while the second stage the phase quantization of the hologram is softly restricted until the desired number of phase levels is reached. The algorithm converges when the result of the iteration improves the RMSE by an amount of $10^{-8}$, compared with the previous one. In the experiment, we put a BBO crystal with dimensions $5 \mathrm{~mm} \times 5 \mathrm{~mm} \times 0.02 \mathrm{~mm}$ at the sample plane, as shown in Fig. 1. The unconverted infrared wavelengths of the ultrashort laser pulse were conveniently filtered with a BG39-Schott crystal before the CCD camera.

At this point, we focus our attention on irradiance rather than phase of the encoded complex field. Hence, in the left part of Fig. 5, we show only the experimental irradiance patterns reconstructed at the sample plane with the two different illumination methods. These patterns were recorded with the camera located at the position shown in Fig. 1, before placing the BBO crystal at the sample plane. From the visual comparison of both images, differences among 
them are apparent. The reconstructed CGH roughly approximates the expected uniform irradiance pattern. Its failed attempt to reproduce a uniform illumination pattern is limited to a clear reinforcement of light at the borders of the ROI, together with the presence of coherent noise. In contrast, the irradiance pattern obtained with our CIM is almost fully flat and free from speckle noise. To better illustrate the differences between both patterns we show the irradiance profile along the central part of the ROIs. The calculated fluctuations with respect to the ideal flat profile are $62 \%$ for the CGH method, against $12 \%$ for the CIM.

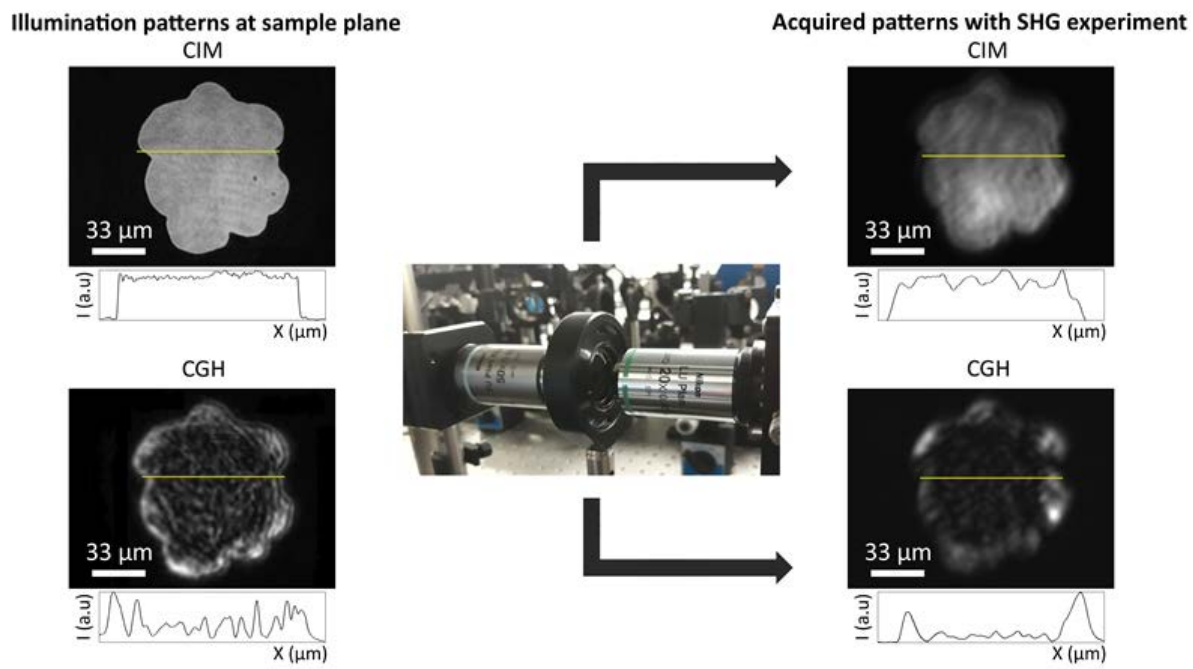

Fig. 5. Experimental images of second harmonic emission from a Daphnia 'eye ROI after employed both CGHs and our illumination method. Scales and central irradiance profiles are included as insets at the left-bottom and right-bottom parts of each irradiance pattern, respectively.

Once the nonlinear medium is placed at the focal plane, we are able to measure second harmonic signal. The corresponding recorded normalized images are shown now in the right part of Fig. 5. As it might be expected, the nonlinear response recorded in this experiment is highly dependent on the degree of flatness of the illumination patterns. That is, second harmonic image due to the illumination with reconstructed CGHs shows almost no light at those sites different from the edges of the ROI. For completeness, we include at the bottom part of each image a spatial profile of these patterns, taken again at the center of each one. This time the fluctuations with respect to the ideal flat profile are $77 \%$ for the CGH method, and $23 \%$ for the CIM.

Last experiments will be aimed to demonstrate how important could the phase control be in nonlinear excitation of ROIs when using the proposed CIM. With this purpose, we select two adjacent and micrometric ROIs located within the Daphnia's eye, which might correspond with different biological tissues in the eye. Then, we excite SHG at both ROIs, simultaneously. In these conditions, after light propagation from the sample plane to a given arbitrary defocusing plane, interference pattern dependence on the phase shift between ROIs can appear [27]. With the help of the CIM, arbitrary phase values can be easily set at different ROIs. For instance, in Fig. 6 we show four images recorded after encoding (only into one of the two ROIs) the phase values $0, \pi / 2, \pi$, and $3 \pi / 2$ radians, respectively. For this example, the selected defocusing plane was located $115 \mathrm{~mm}$ away from the sample plane. The recorded images shown in Fig. 6 indicate the existence of interference patterns whose structure depends on the phase shift between ROIs. Main differences among interference patterns are seen near the border of the two adjacent ROIs where the light is coherent mixed. 
a)
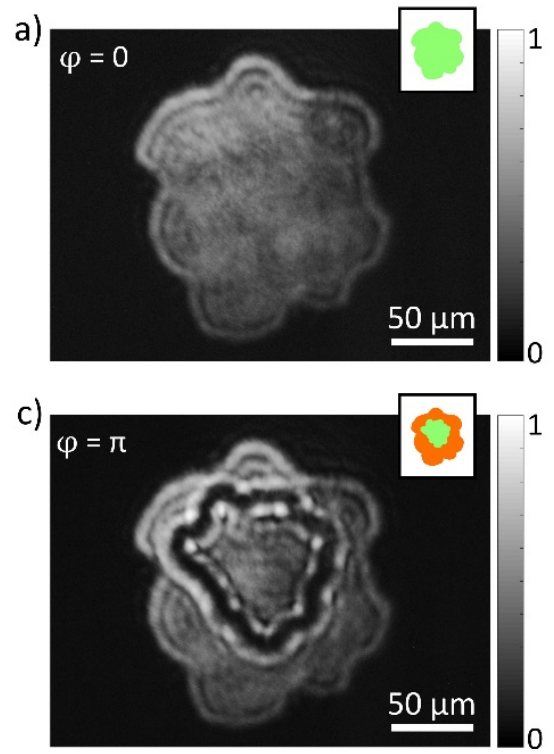
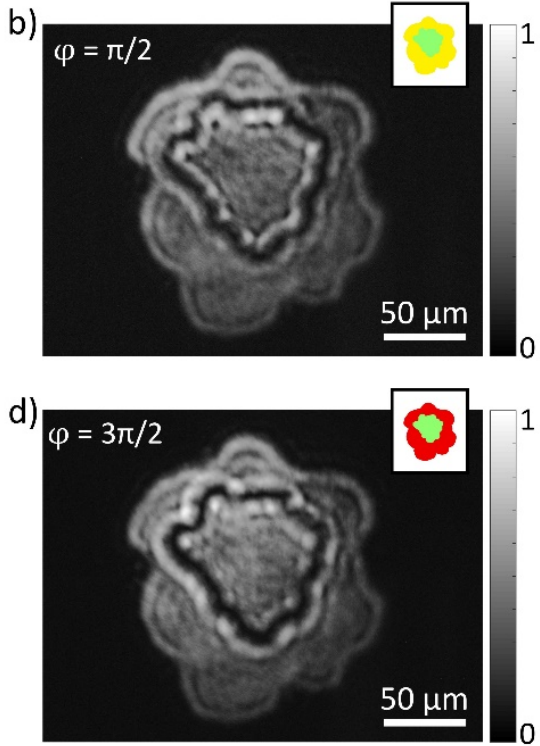

Fig. 6. Interference pattern dependence on the phase set at one ROI. The spatial shape of the selected ROIs are indicated with different colors and included as insets at the right-top part of each image.

To avoid interference border effects, we carried out a complementary experiment. This time the selected micrometric ROIs are isolated by a black surrounding ring between them such that, at the image plane, they do not interfere at all, see the right-top part of Fig. 7. To help light propagating from both ROIs to interfere, an additional convergent lens of $100 \mathrm{~mm}$ focal length was placed after the image plane. The position of the camera was set at an arbitrary defocusing z-axis plane located $140 \mathrm{~mm}$ after the Fourier plane of the added lens. In these conditions, we measured a sequence of defocusing images, each one corresponding to the setting (by means of the CIM) of a different phase value (from 0 to $2 \pi$ ) at one ROI, while keeping the phase at the other ROI unchanged. With all recorded images we made a movie of the evolution of the interference pattern as the phase at one ROI is increased, please see Fig. 7. Now, after watching the movie one can corroborate the presence of clear interference fringes at the previously-defined black surrounding ring. In addition, one can realize that the spatial location of these fringes depends on the phase shift between ROIs.

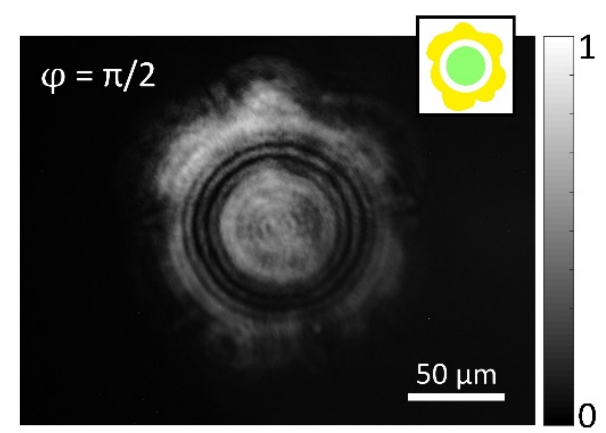

Fig. 7. Defocusing image after exciting SHG from two micrometric and separated ROIs with a set phase shift between them. The spatial shape of the selected ROIs are indicated with different colors and included as insets at the right-top part of the image. The light propagating from the ROIs interacts with one another and generates interference fringes (see Visualization 1). 
From a practical point of view, last two experiments suggest that recorded SHG interference patterns might give us information on the relative phase between ROIs in real biological samples. Therefore, in addition to amplitude modulation, the ability of the CIM to manipulate the phase at the selected ROIs allows for practical benefits (e.g., optical aberration corrections, reduction of zero order light, or interferometric-based phase information retrieval).

\section{General considerations}

In this section we will discuss on significant practical issues of the CIM. For instance, in order to highly mitigate the effect of non-diffracted light (zero order) onto the sample plane, the light in the ODM enters the microscope objective $\mathrm{MO}_{1}$ under low-focusing, instead of typical planewave configuration. In this way, non-diffracted light exits the microscope objective parallel to the optical axis without forming any potentially-dangerous focus near the output plane of the uf optical system (sample plane). Hence, at the sample plane the excitation pattern is determined by the light diffracted at the SLM and the zero-order contribution. At this point, we measured the average power at the sample plane after encoding a uniform circular amplitude pattern with and without a blazed grating (with maximum grating efficiency of $80 \%$ at the first diffraction order when is codified with 4 discrete phase levels). This allows us to estimate the ratio between non-diffracted and diffracted light in the current on-axis optical arrangement. Specifically, we found a ratio of $41 \%$. Obviously, for an off-axis optical arrangement, the zero order light can be almost completely eliminated from the sample plane with the encoding of a proper blazed grating, together with the desired complex field. The same procedure was repeated employing a holographic scheme. This time we found a ratio of $44 \%$. In addition, thanks to the use of a long-distance focal length achromatic lens to direct the light into the microscope objective $\mathrm{MO}_{1}$, the effects of spherical aberration at the sample plane can be disregarded. Perhaps, this can be better understood in terms of the produced Rayleigh range inside the microscope objective, which is longer and longer as far as we increase the focal length of above-mentioned achromatic lens.

Now we analyze the efficiency of the CIM. In general the efficiency of a method based on encoding information onto a SLM depends on several factors, including but not limited to technical specifications of the SLM, the type of information (amplitude or phase) that one needs to encode, characteristics of the light source, and/or the orientation of the SLM with respect to the incident light (oblique or straight light incident). In the proposed illumination method main energy losses come from the diffraction of light at the phase element, as well as due to the physical specifications of our SLM (in accordance with the manufacturer: $80 \%$ diffraction efficiency, 65-95\% reflectivity, 93\% fill factor, so the total light efficiency can be more than 50\%). Another important issue to consider here is the size of the encoding patterns, which is directly proportional to the efficiency of the method. In other words, as in our illumination method the SLM plane is (except for the spatial filtering process) conjugated to the sample plane, an increase in the area of the encoded pattern at the SLM leads to more available light for illumination purposes. In this context, we measured the efficiency of the illumination method for the generation of irradiance patterns with the spatial shape of circumferences having different radii, and compared these results with similar ones obtained from the reconstruction of CGHs with equal shapes. To do that, we calculate the ratio of the average laser beam powers measured at the output plane of the CFEM and before the SLM with the help of a power meter (GENTEC Tuner). Our results confirm that, apart from common energy losses, the efficiency of our method is pattern-size dependent. For instance, when the encoded pattern utilizes less than $10 \%$ of the active area (about $133 \mathrm{~mm}^{2}$ ) of the SLM, the classical CGH-based illumination method is more efficient than ours (for instance for encoded pattern dimensions of about $8 \mathrm{~mm}^{2}$, we get $18 \%$ efficiency against $23 \%$ of the classical method). In contrast, when encoded patterns fill out areas greater than $25 \%$ of the SLM display area which is perhaps a more useful scenario from an energy point of view, the efficiency of 
our method exceeds that of the classical one. For instance for encoded pattern dimensions of about $60 \mathrm{~mm}^{2}$, we get $32 \%$ efficiency of our method against $23 \%$ of the classical one.

Another important point of the CIM is its potential to perform wide-field illumination and nonlinear excitation of biological samples without damage of optical components. On this aspect, the critical component of the ODM is clearly the microscope objective $\mathrm{MO}_{1}$ that is working under low but focusing incident light conditions. The use of long-distance focal lengths certainly allows light energy inside the microcopy objective to be axially distributed along the associated Rayleigh range, decreasing in this way potential optical damage in comparison with the use of shorter focal length lenses. We measured the threshold average beam power at which nonlinear effects appear in the microscope objective. This value was about $20 \mathrm{~mW}$, which turned out to be relative far from the typical average powers (4-10 $\mathrm{mW}$ ) that we use in our experiments.

Finally we discuss on the dispersion compensation capabilities of the CIM. In the propagation of ultrashort pulses, even through non-dispersive media, there is a coupling of spatial and temporal effects due to the wavelength dependence of the diffraction integral. However, for the propagation between input and output planes of image systems (as the ones reported in this manuscript), the diffraction integral is no longer wavelength dependent and consequently, the spatiotemporal coupling no longer exists. All the distortions that appear at the output plane of our system will be just the ones introduced by the optical components, but not by the diffraction itself. So, in first order approximation, the distortions in the spectral phase of the ultrashort pulse can be pre-compensated with a common dispersion compensation module. In our case, we employed a commercially available module (Dazzler) installed in the amplification stage of our femtosecond laser. For instance, temporal pulse stretching is mainly mitigated with a proper combination of second and third order terms if we look at the maximum second harmonic signal of a BBO crystal at the sample plane. Additionally, when necessary, optical aberrations at the sample plane can also be partially compensated by encoding a suited phase mask (made up of a set of weighted aberration Zernike polynomials) together with phase element $\alpha(x, y)$ given in Eq. (4). Note that, the lack of significant dispersion contributions at the sample plane can be regarded as an advantage of our proposal in comparison with other illumination strategies e.g., CGHs.

\section{Conclusions}

In this manuscript we have proposed and tested an interferometric CIM mainly addressed to microscopy applications which is able to achieve simultaneous linear and/or non-linear excitation of user-defined ROIs. The introduced CIM provides not only full control over the amplitude and phase of the complex field at the sample plane, but also reduces speckled noise due to the use of coherent illumination sources. The complex illumination pattern is basically generated from a single phase element (calculated from the double-phase hologram theory) and encoded into a phase-only SLM. In the optical setup made up of a couple of modules called CFEM and ODM, the light coming from the SLM is spatially filtered and demagnified with proper optical components before arriving to the sample plane.

In the manuscript we have discussed both the theory underlying the CIM as well as its experimental performance, including zero order behavior, dispersion compensation capabilities or efficiency. In practice, the proposed CIM has been validated with several experiments aimed to produce linear and non-linear excitation of specific ROIs with userdefined complex illumination patterns. In addition some benefits of our CIM over other illumination schemes based on IFTAs and implemented with phase-only SLMs have been highlighted with practical demonstrations related to aberration compensation or interferometric-based phase information retrieval tasks.

Our experiments show that in principle, under fully controllable amplitude and phase parameters, not only linear but also second harmonic excitation of different ROIs at 
micrometric scale is feasible. Here, it should be mentioned that these experiments can only be considered as a proof-of-concept. It is apparent that additional tests must be done in order to validate the proposed CIM i.e., use it as the illumination path of a commercial microscope and/or by studying its behavior under real biological samples. However, we do believe that achieved results should be enough to show the potential of the proposed CIM for microscopy applications. For instance, the probed ability of the CIM to simultaneous excite spatiallyextended ROIs and simultaneously compensate for optical aberrations might positively contribute to decrease the acquisition time and/or improve the spatial resolution of acquired images. To this end, a proper combination of the proposed CIM with high-sensitivity cameras is expected. Moreover, to enhance axial resolution, CIM can be combined with well-known temporal focusing methods $[8,20]$.

\section{Funding}

Generalitat Valenciana (AICO/2016/036, PROMETEO 2016-079); Universitat Jaume I (UJI) (UJIB2016-19); Ministerio de Economía y Competitividad (MINECO) (FIS2016-75618-R).

\section{Acknowledgments}

The authors are very grateful to the SCIC of the Universitat Jaume I for the use of the femtosecond laser.

\section{References}

1. C. K. Kim, S. J. Yang, N. Pichamoorthy, N. P. Young, I. Kauvar, J. H. Jennings, T. N. Lerner, A. Berndt, S. Y. Lee, C. Ramakrishnan, T. J. Davidson, M. Inoue, H. Bito, and K. Deisseroth, "Simultaneous fast measurement of circuit dynamics at multiple sites across the mammalian brain,” Nat. Methods 13(4), 325-328 (2016).

2. M. Ducros, Y. Goulam Houssen, J. Bradley, V. de Sars, and S. Charpak, "Encoded multisite two-photon microscopy,” Proc. Natl. Acad. Sci. U.S.A. 110(32), 13138-13143 (2013).

3. M. T. Butko, M. Drobizhev, N. S. Makarov, A. Rebane, B. C. Brinkman, and J. G. Gleeson, "Simultaneous multiple-excitation multiphoton microscopy yields increased imaging sensitivity and specificity,” BMC Biotechnol. 11(1), 20 (2011).

4. M. Ingaramo, A. G. York, P. Wawrzusin, O. Milberg, A. Hong, R. Weigert, H. Shroff, and G. H. Patterson, "Two-photon excitation improves multifocal structured illumination microscopy in thick scattering tissue," Proc. Natl. Acad. Sci. U.S.A. 111(14), 5254-5259 (2014).

5. A. Egner and S. W. Hell, “Time multiplexing and parallelization in multifocal multiphoton microscopy,” J. Opt. Soc. Am. A 17(7), 1192-1201 (2000).

6. S. Lévêque-Fort, M. P. Fontaine-Aupart, G. Roger, and P. Georges, "Fluorescence-lifetime imaging with a multifocal two-photon microscope,” Opt. Lett. 29(24), 2884-2886 (2004).

7. E. Papagiakoumou, V. de Sars, D. Oron, and V. Emiliani, "Patterned two-photon illumination by spatiotemporal shaping of ultrashort pulses,” Opt. Express 16(26), 22039-22047 (2008).

8. L.-C. Cheng, C.-Y. Chang, C.-Y. Lin, K.-C. Cho, W.-C. Yen, N.-S. Chang, C. Xu, C. Y. Dong, and S.-J. Chen, "Spatiotemporal focusing-based widefield multiphoton microscopy for fast optical sectioning," Opt. Express 20(8), 8939-8948 (2012).

9. M. D. Peterson, P. L. Hayes, I. S. Martinez, L. C. Cass, J. L. Achtyl, E. A. Weiss, and F. M. Geiger, "Second harmonic generation imaging with a kHz amplifier [Invited]," Opt. Mater. Express 1(1), 57-66 (2011).

10. V. Nikolenko, D. S. Peterka, R. Araya, A. Woodruff, and R. Yuste, "Spatial light modulator microscopy," Cold Spring Harb. Protoc. 2013(12), 1132-1141 (2013).

11. Y. Hertzberg, O. Naor, A. Volovick, and S. Shoham, "Towards multifocal ultrasonic neural stimulation: pattern generation algorithms,” J. Neural Eng. 7(5), 056002 (2010).

12. O. Mendoza-Yero, G. Mínguez-Vega, and J. Lancis, "Encoding complex fields by using a phase-only optical element," Opt. Lett. 39(7), 1740-1743 (2014).

13. E. Papagiakoumou, F. Anselmi, A. Bègue, V. de Sars, J. Glückstad, E. Y. Isacoff, and V. Emiliani, "Scanless two-photon excitation of channelrhodopsin-2,” Nat. Methods 7(10), 848-854 (2010).

14. I. Pastirk, J. Dela Cruz, K. Walowicz, V. Lozovoy, and M. Dantus, "Selective two-photon microscopy with shaped femtosecond pulses,” Opt. Express 11(14), 1695-1701 (2003).

15. C. Maurer, A. Jesacher, S. Bernet, and M. Ritsch-Marte, "What spatial light modulators can do for optical microscopy,” Laser Photonics Rev. 5(1), 81-101 (2011).

16. S. Bovetti and T. Fellin, "Optical dissection of brain circuits with patterned illumination through the phase modulation of light," J. Neurosci. Methods 241, 66-77 (2015).

17. D. Tanese, J.-Y. Weng, V. Zampini, V. De Sars, M. Canepari, B. Rozsa, V. Emiliani, and D. Zecevic, "Imaging membrane potential changes from dendritic spines using computer-generated holography,” Neurophotonics 4(3), 031211 (2017) 


\section{Optics EXPRESS}

18. A. Jesacher, C. Maurer, A. Schwaighofer, S. Bernet, and M. Ritsch-Marte, "Near-perfect hologram reconstruction with a spatial light modulator," Opt. Express 16(4), 2597-2603 (2008).

19. S. J. Yang, W. E. Allen, I. Kauvar, A. S. Andalman, N. P. Young, C. K. Kim, J. H. Marshel, G. Wetzstein, and K. Deisseroth, "Extended field-of-view and increased-signal 3D holographic illumination with time-division multiplexing," Opt. Express 23(25), 32573-32581 (2015).

20. N. Accanto, C. Molinier, D. Tanese, E. Ronzitti, Z. L. Newman, C. Wyart, E. Isacoff, E. Papagiakoumou, and V. Emiliani, "Multiplexed temporally focused light shaping for high-resolution multi-cell targeting," Optica 5(11), 1478-1491 (2018).

21. R. W. Gerchberg and W. O. Saxton, "A practical algorithm for the determination of phase from image and diffraction plane pictures,” Optik (Stuttg.) 35(2), 237-246 (1972).

22. T. Aharoni and S. Shoham, "Phase-controlled, speckle-free holographic projection with applications in precision optogenetics,” Neurophotonics 5(2), 025004 (2018).

23. R. Conti, O. Assayag, V. de Sars, M. Guillon, and V. Emiliani, "Computer generated holography with intensitygraded patterns,” Front. Cell. Neurosci. 10, 236 (2016).

24. O. Mendoza-Yero, G. Minguez-Vega, L. Martinez-Leon, M. Carbonell-Leal, M. Fernandez-Alonso, C. DonateBuendia, J. Perez-Vizcaino, and J. Lancis, "Diffraction-based phase calibration of spatial light modulators with binary phase Fresnel lenses,” J. Disp. Technol. 12(10), 1027-1032 (2016).

25. M. Persson, D. Engström, and M. Goksör, "Reducing the effect of pixel crosstalk in phase only spatial light modulators,” Opt. Express 20(20), 22334-22343 (2012).

26. I. Yamaguchi and T. Zhang, "Phase-shifting digital holography,” Opt. Lett. 22(16), 1268-1270 (1997).

27. R. Bäumner, L. Bonacina, J. Enderlein, J. Extermann, T. Fricke-Begemann, G. Marowsky, and J. P. Wolf, "Evanescent-field-induced second harmonic generation by noncentrosymmetric nanoparticles,” Opt. Express 18(22), 23218-23225 (2010). 